\title{
Additional Protocol (AP) Protocol Reporter Version 3.0 (PR3) System Design Specification (SDS)
}

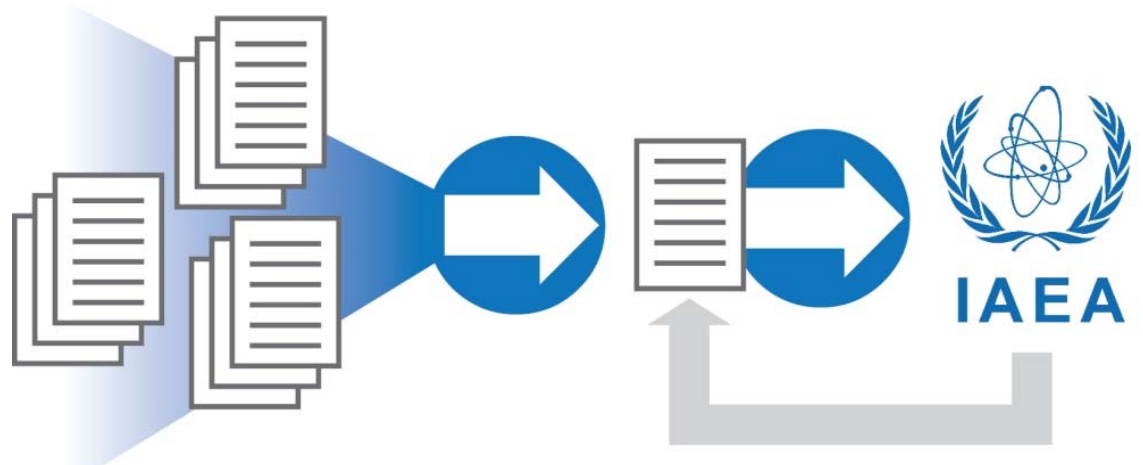

Chad Braden

Ron Cain

Kevin Hannan

Jacqueline Idinger

Donald Kovacic

Wilhelm Mandl

Alain Rialhe

Dylan Wolf

August 2015 


\title{
DOCUMENT AVAILABILITY
}

Reports produced after January 1, 1996, are generally available free via US Department of Energy (DOE) SciTech Connect.

Website http://www.osti.gov/scitech/

Reports produced before January 1, 1996, may be purchased by members of the public from the following source:

\author{
National Technical Information Service \\ 5285 Port Royal Road \\ Springfield, VA 22161 \\ Telephone 703-605-6000 (1-800-553-6847) \\ TDD 703-487-4639 \\ Fax 703-605-6900 \\ E-mail info@ntis.gov \\ Website http://www.ntis.gov/help/ordermethods.aspx
}

Reports are available to DOE employees, DOE contractors, Energy Technology Data Exchange representatives, and International Nuclear Information System representatives from the following source:

Office of Scientific and Technical Information

PO Box 62

Oak Ridge, TN 37831

Telephone 865-576-8401

Fax 865-576-5728

E-mail reports@osti.gov

Website http://www.osti.gov/contact.html

This report was prepared as an account of work sponsored by an agency of the United States Government. Neither the United States Government nor any agency thereof, nor any of their employees, makes any warranty, express or implied, or assumes any legal liability or responsibility for the accuracy, completeness, or usefulness of any information, apparatus, product, or process disclosed, or represents that its use would not infringe privately owned rights. Reference herein to any specific commercial product, process, or service by trade name, trademark, manufacturer, or otherwise, does not necessarily constitute or imply its endorsement, recommendation, or favoring by the United States Government or any agency thereof. The views and opinions of authors expressed herein do not necessarily state or reflect those of the United States Government or any agency thereof. 
Nuclear Security and Isotope Technology Division

\title{
ADDITIONAL PROTOCOL (AP) PROTOCOL REPORTER VERSION 3.0 (PR3) SYSTEM DESIGN SPECIFICATION (SDS)
}

\author{
Chad Braden \\ Ron Cain \\ Kevin Hannan* \\ Jacqueline Idinger \\ Donald Kovacic \\ Wilhelm Mandl \\ Alain Rialhe \\ Dylan Wolf*
}

*Trident Resource Corporation

Date Published: August 2015

\author{
Prepared by \\ OAK RIDGE NATIONAL LABORATORY \\ Oak Ridge, TN 37831-6283 \\ managed by \\ UT-BATTELLE, LLC \\ for the \\ US DEPARTMENT OF ENERGY \\ under contract DE-AC05-00OR22725
}





\section{CONTENTS}

\begin{tabular}{|c|c|c|}
\hline & & Page \\
\hline ST O & FIGUF & ....................vii \\
\hline LIST O & TABL & .....ix \\
\hline ACRON & MS. & .........xi \\
\hline 1. INT & RODUC & - \\
\hline 1.1 & PURP & 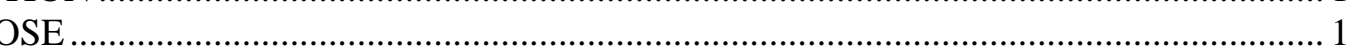 \\
\hline 1.2 & TARG & 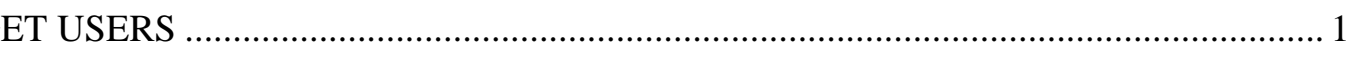 \\
\hline 1.3 & USAG & 2 \\
\hline 1.4 & ENVIF & 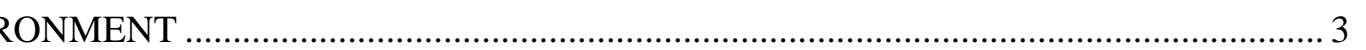 \\
\hline 1.5 & USER & 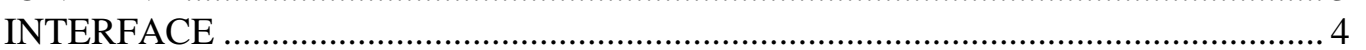 \\
\hline 1.6 & INPUT & TS AND OUTPUTS .................. \\
\hline 1.7 & OTHE & R RESOURCES \\
\hline 2. USF & R INTE & RFACE DESIGN \\
\hline 2.1 & USER & 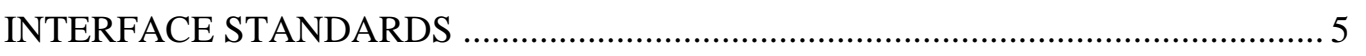 \\
\hline & 2.1 .1 & Visual Theme \\
\hline & 2.1 .2 & 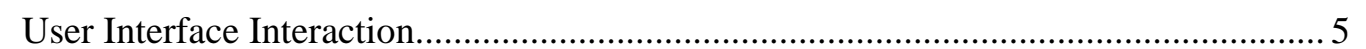 \\
\hline & 2.1 .3 & User Interface Controls ........................... \\
\hline & 2.1 .4 & 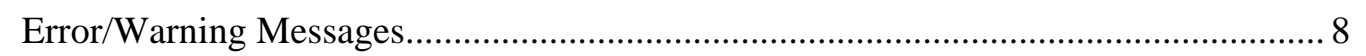 \\
\hline & 2.1 .5 & 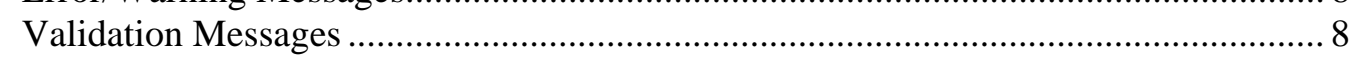 \\
\hline & 2.1 .6 & Wizards \\
\hline & 2.1 .7 & Reports \\
\hline & 2.1 .8 & 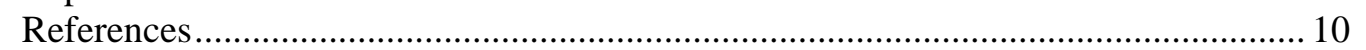 \\
\hline & 2.1 .9 & Documents \\
\hline 2.2 & USER & 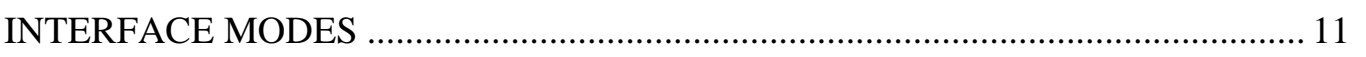 \\
\hline & 2.2 .1 & 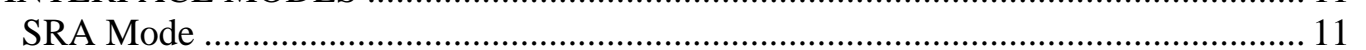 \\
\hline & 2.2 .2 & Contributor Mode \\
\hline 2.3 & USER & INTERFACE WORKFLOWS............. \\
\hline & 2.3 .1 & 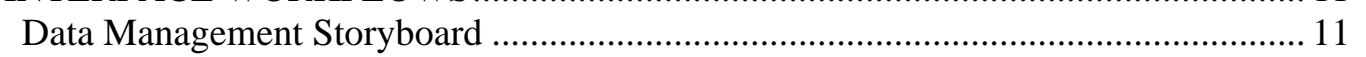 \\
\hline & 2.3 .2 & Entry Editing \\
\hline & 2.3 .3 & 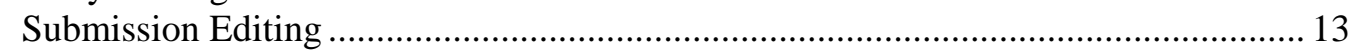 \\
\hline & 2.3 .4 & 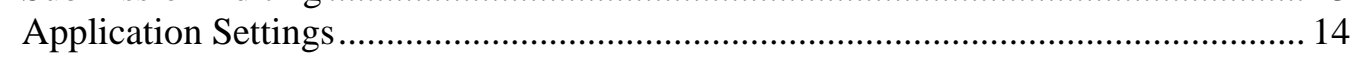 \\
\hline 2.4 & USER & INTERFACE LAYOUTS \\
\hline & 2.4 .1 & 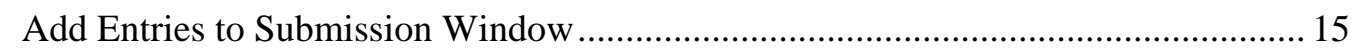 \\
\hline & 2.4 .2 & 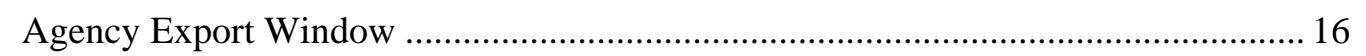 \\
\hline & 2.4 .3 & 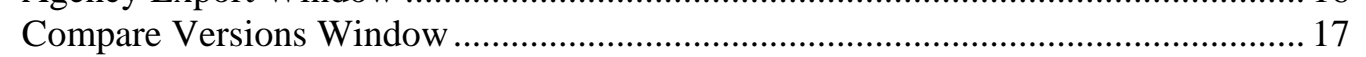 \\
\hline & 2.4 .4 & 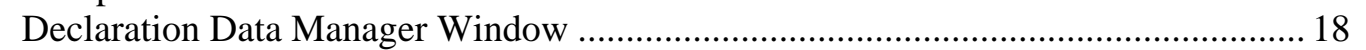 \\
\hline & 2.4 .5 & 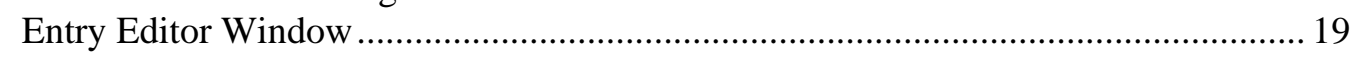 \\
\hline & 2.4 .6 & 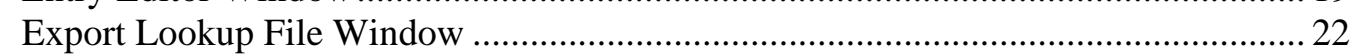 \\
\hline & 2.4 .7 & 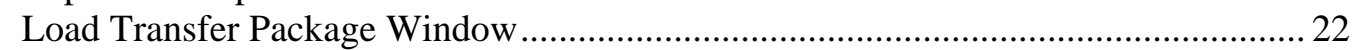 \\
\hline & 2.4 .8 & 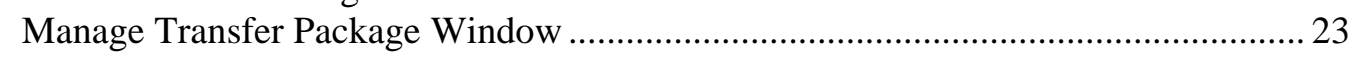 \\
\hline & 2.4 .9 & 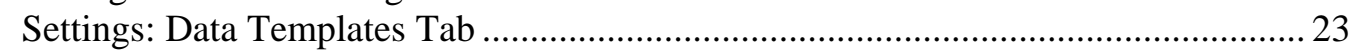 \\
\hline & 2.4 .10 & 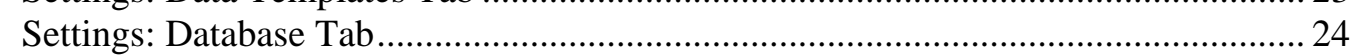 \\
\hline & 2.4.11 & 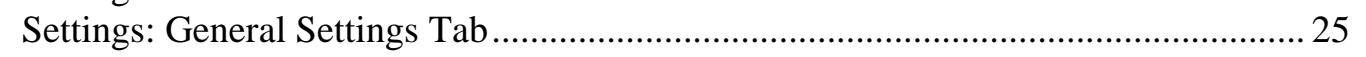 \\
\hline & 2.4 .12 & Settings: Lookup Data Tab ...................... \\
\hline & 2.4 .13 & 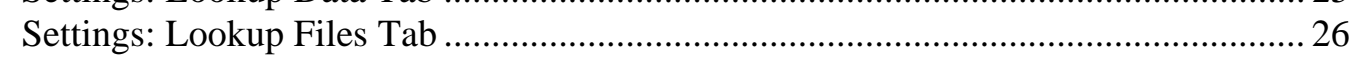 \\
\hline & 2.4.14 & 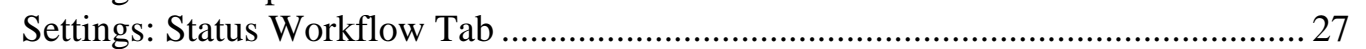 \\
\hline & 2.4 .15 & 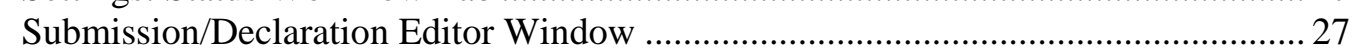 \\
\hline
\end{tabular}




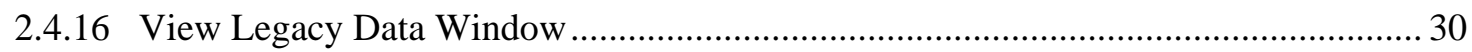

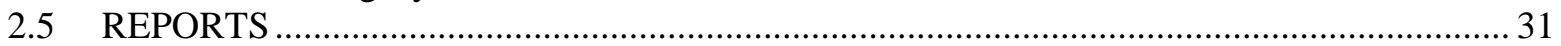

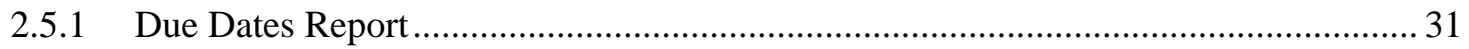

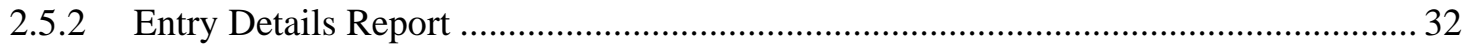

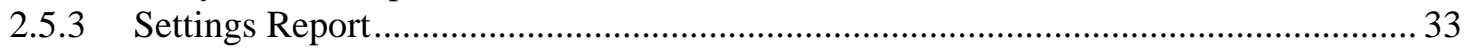

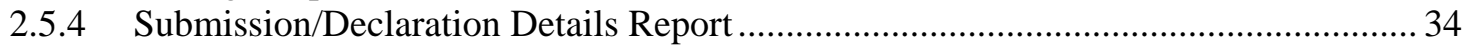

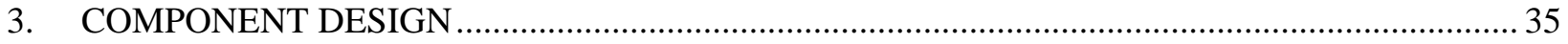

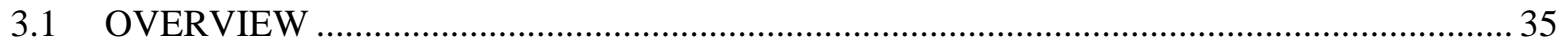

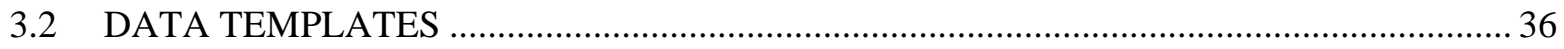

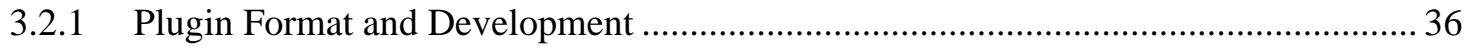

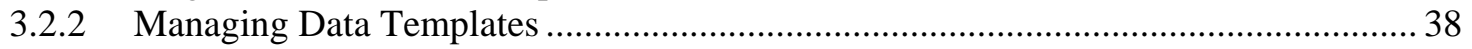

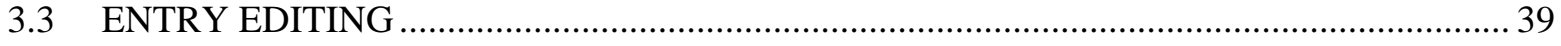

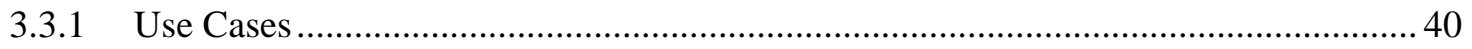

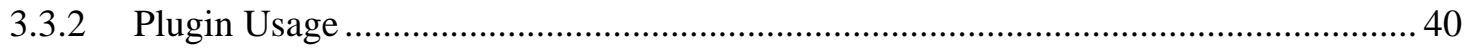

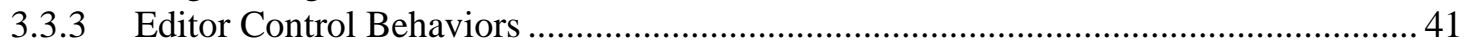

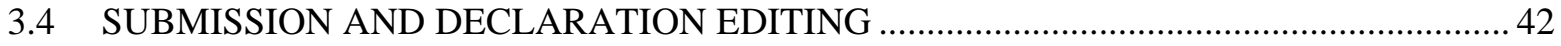

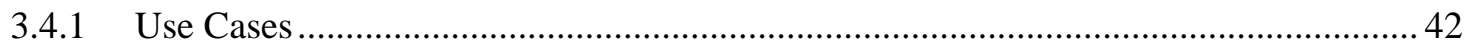

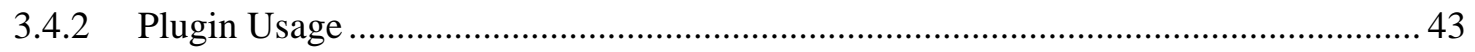

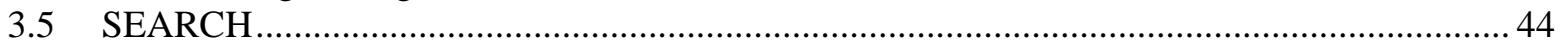

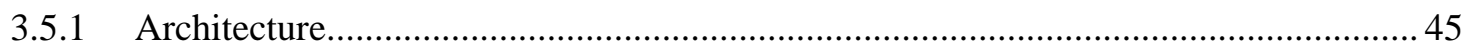

3.5.2 Third-party Library Requirements and Recommendations.......................................... 45

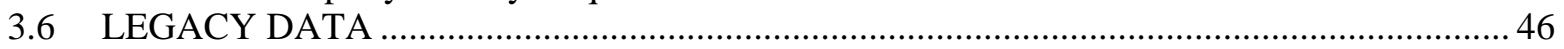

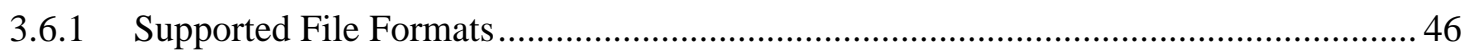

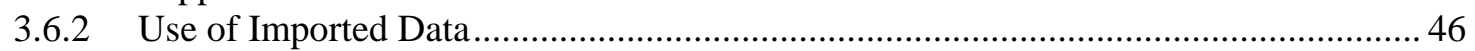

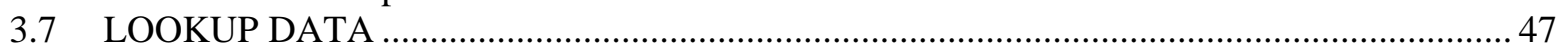

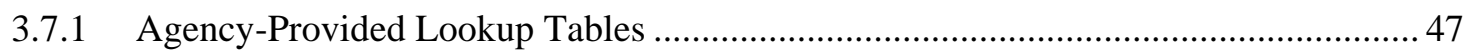

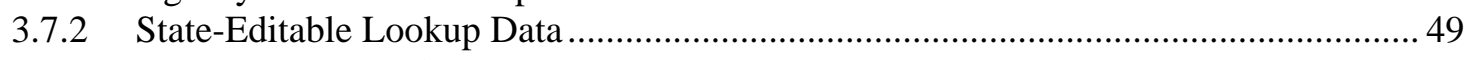

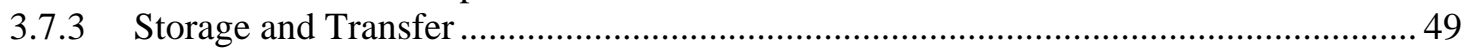

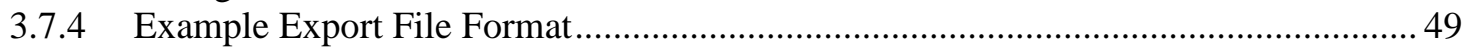

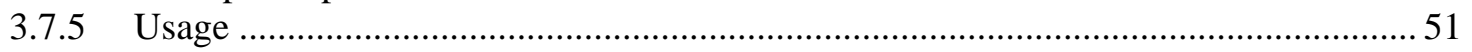

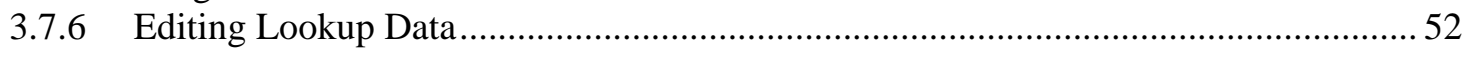

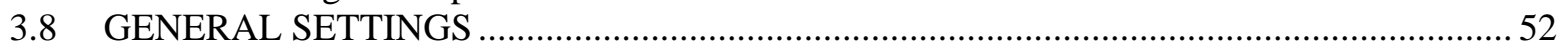

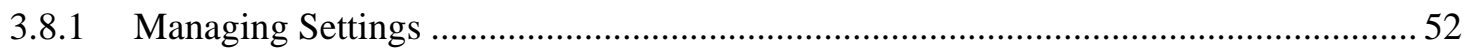

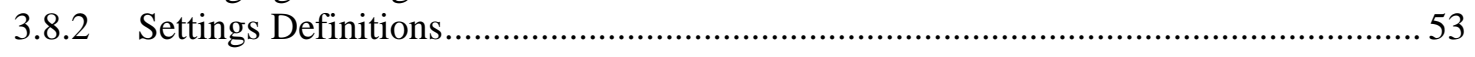

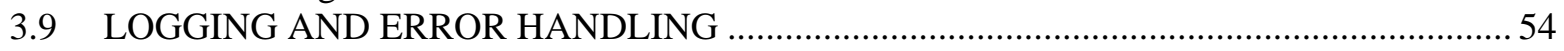

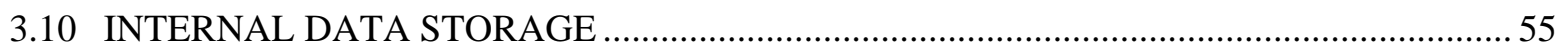

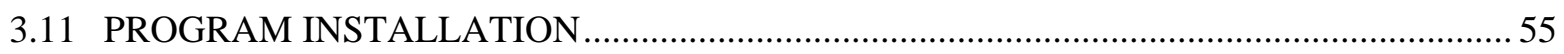

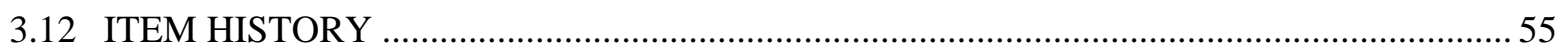

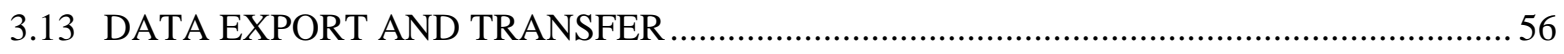

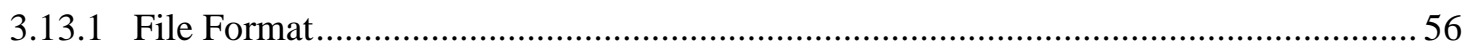

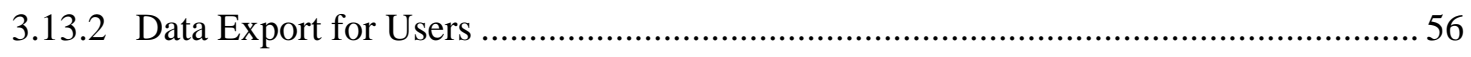

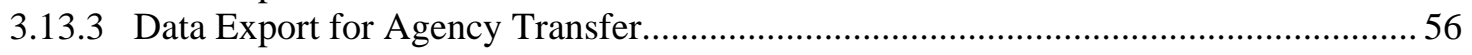

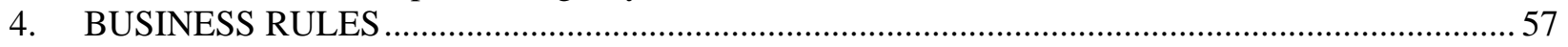

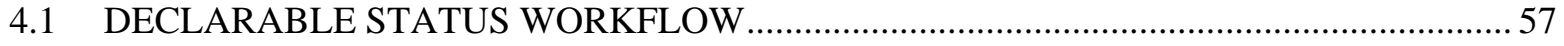

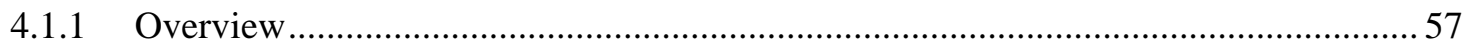

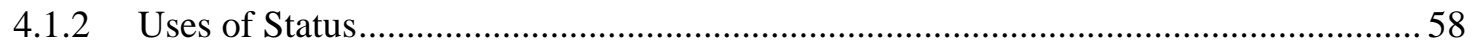

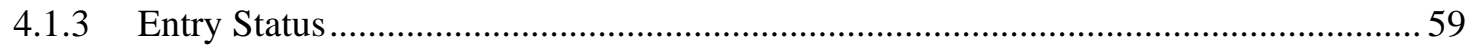

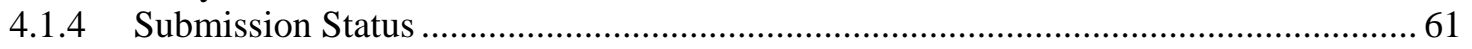

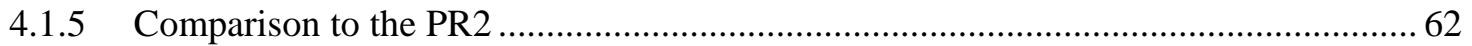

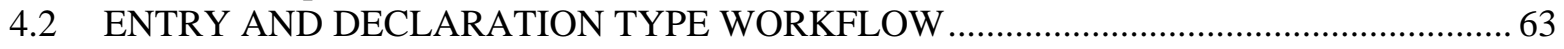

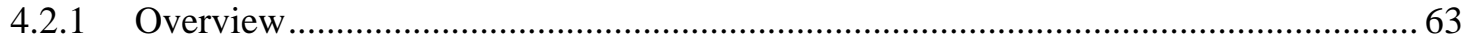




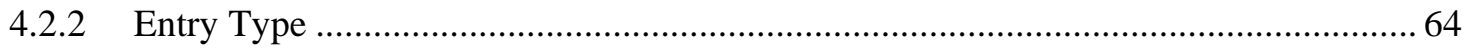

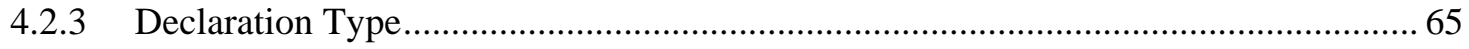

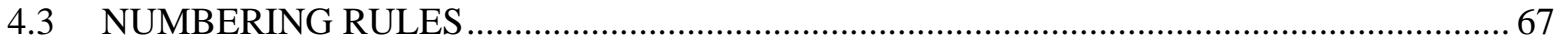

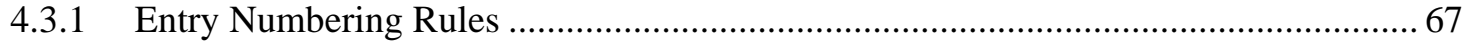

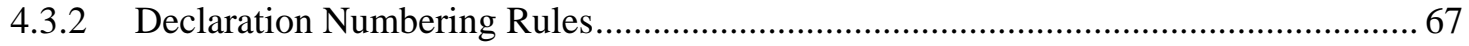

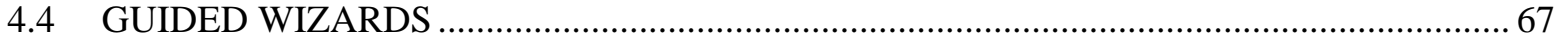

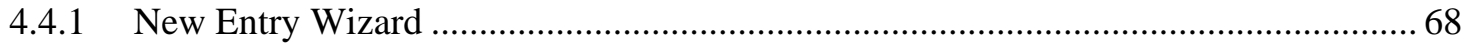

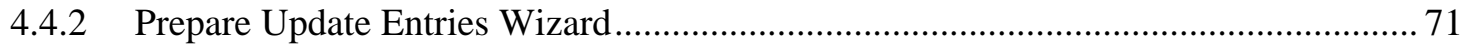

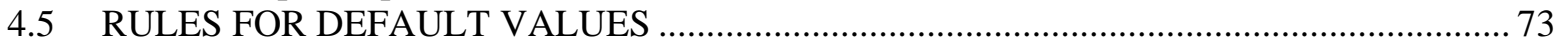

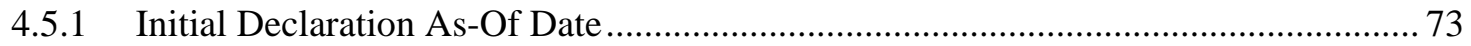

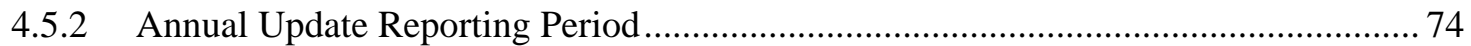

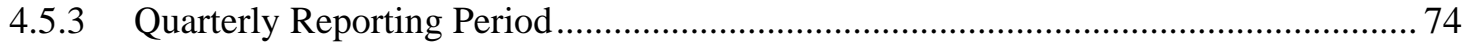

4.5.4 Submission Starting Declaration Number................................................................ 75

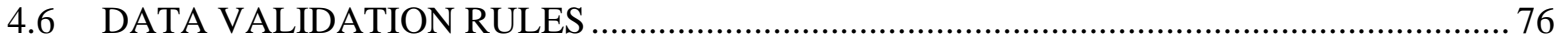

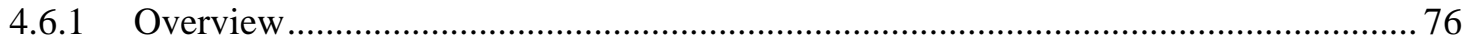

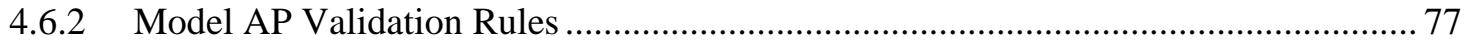

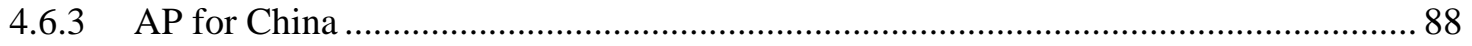

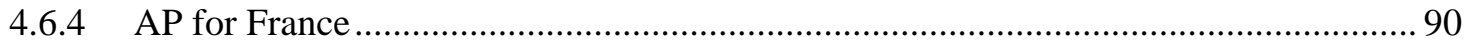

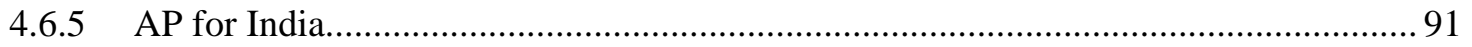

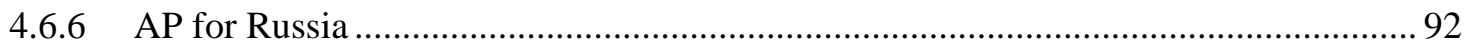

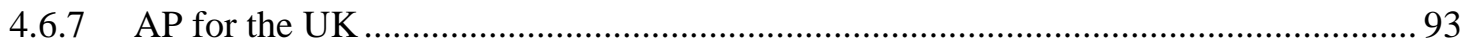

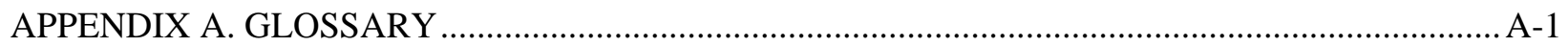

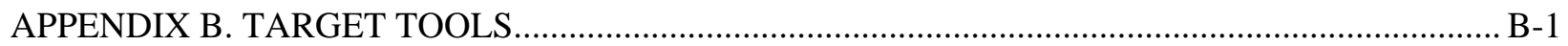

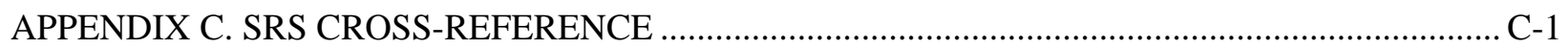

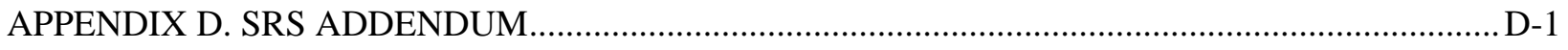

APPENDIX E. PR3 VISUAL PROTOTYPE OVERVIEW …......................................................... E-1 



\section{LIST OF FIGURES}

Figure

Fig. 1. Example workflow for a State with contributor organizations.................................................. 3

Fig. 2. Example workflow for a State without contributor organizations.................................................... 3

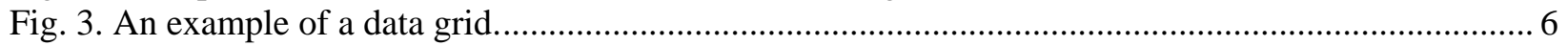

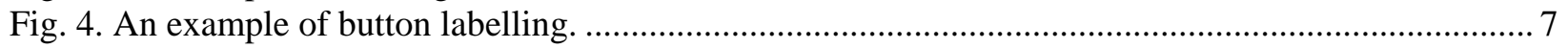

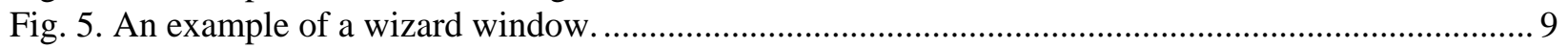

Fig. 6. The windows and interfaces used for Declaration Data Management. ........................................ 12

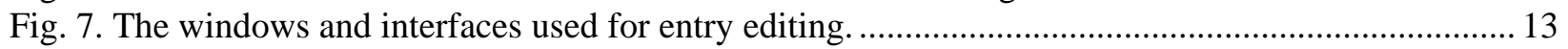

Fig. 8. The windows and interfaces used for submission editing. ....................................................... 14

Fig. 9. The windows and interfaces used for managing settings. ........................................................... 15

Fig. 10. The Eligible Entries tab in the Add Entries to Submission window............................................. 16

Fig. 11. The "Ineligible Entries" tab in the "Add Entries to Submission" window.................................... 16

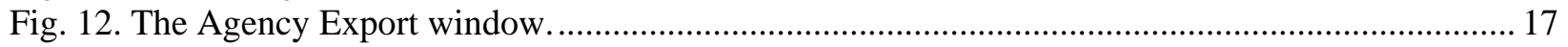

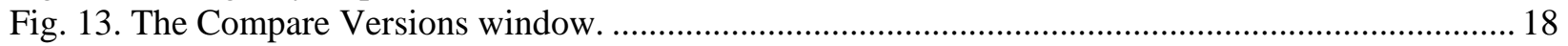

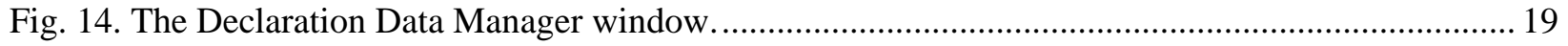

Fig. 15. The Entry Editor window, with the Internal Data tab selected.................................................... 20

Fig. 16. The Entry Editor window, with the References tab selected...................................................... 21

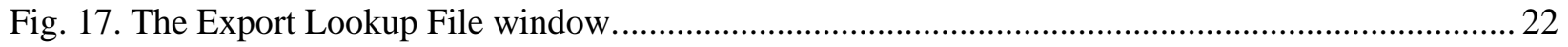

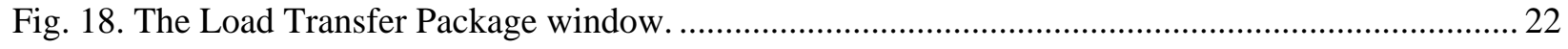

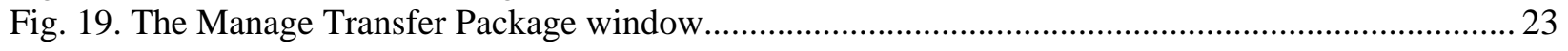

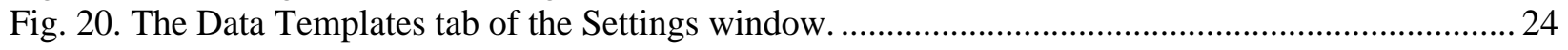

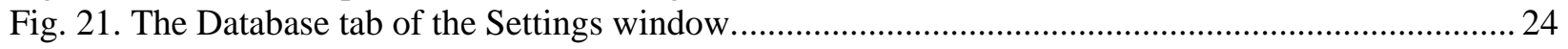

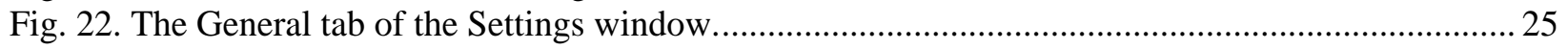

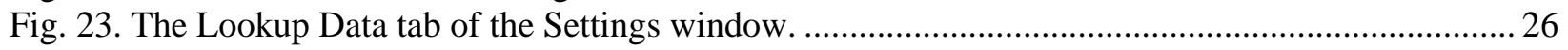

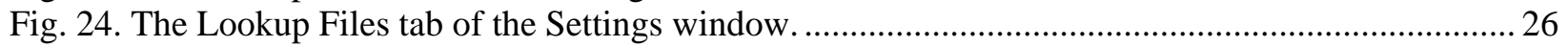

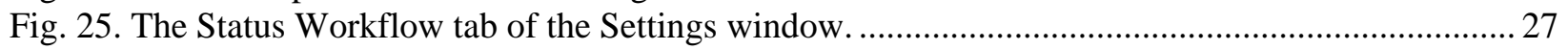

Fig. 26. The Submission Editor with a Declarations Entries tab selected. .............................................. 28

Fig. 27. The Submission Editor with a Declaration's Declaration Details tab selected............................ 29

Fig. 28. The Submission Editor with a Declaration's Internal Comments tab selected. ............................ 30

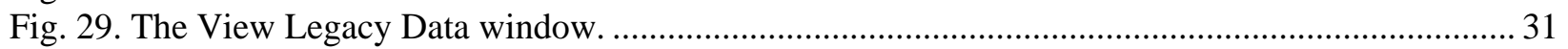

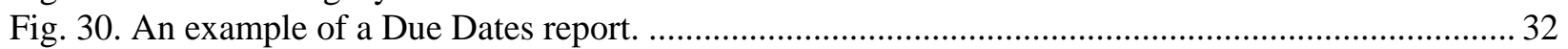

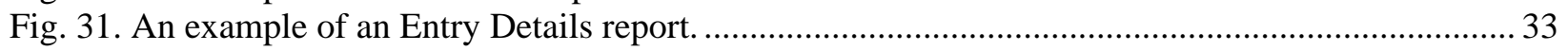

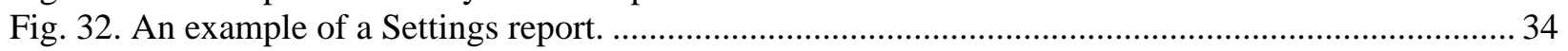

Fig. 33. An example of a Submission Details report. ......................................................................... 35

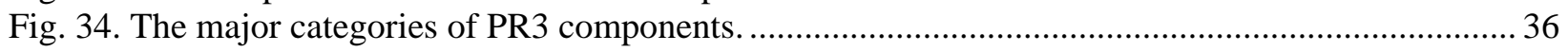

Fig. 35. An example of how assemblies/libraries and classes in the PR3 should relate to support

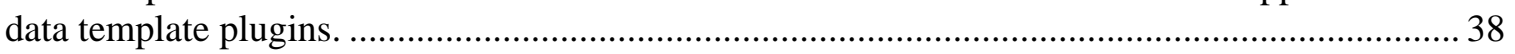

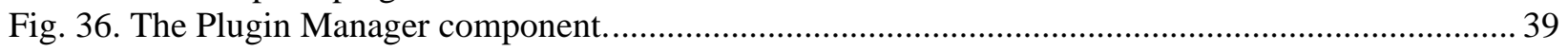

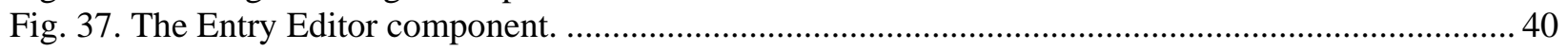

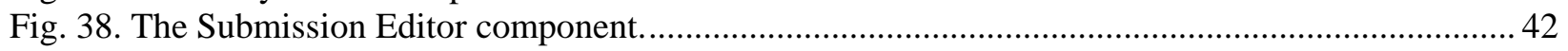

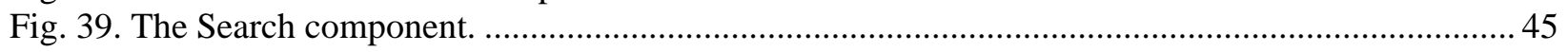

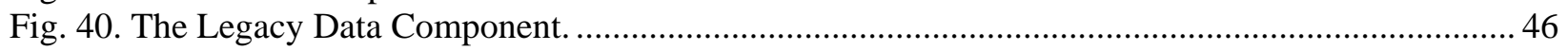

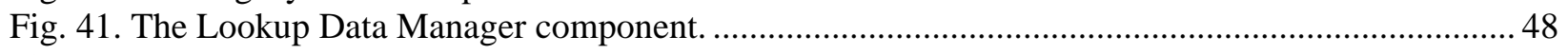

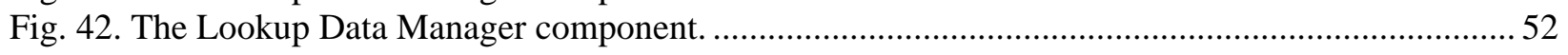

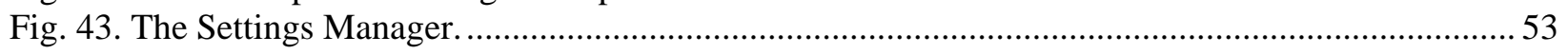

Fig. 44. The roles users may play in a PR3 workflow and what statuses represent to those roles. ............ 58

Fig. 45. A complete workflow that uses all available Entry Statuses................................................... 60 
Fig. 46. A complete workflow that uses all available Submission Statuses. .......................................... 61

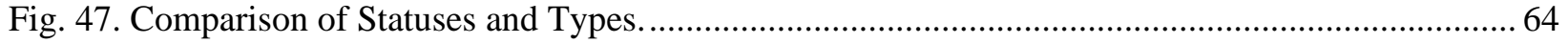

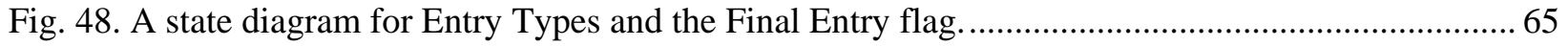

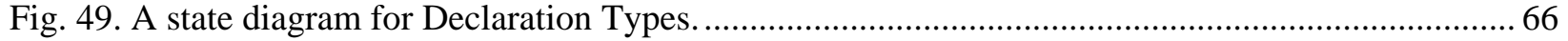




\section{LIST OF TABLES}

\section{Table}

Page

Table 1. Types of user involvement and knowledge required ............................................................. 2

Table 2. Examples of functions that a plugin must provide to the Entry Editor ......................................... 41

Table 3. Examples of plugin functions used by the Submission Editor................................................... 44

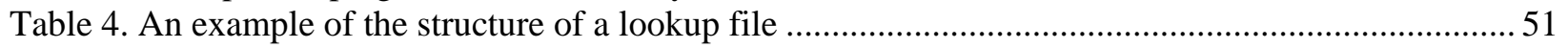

Table 5. An example of how lookup file reading errors should be handled .......................................... 51

Table 6. The list of Settings that must be included in PR3 …............................................................. 53

Table 7. The roles users may play in a PR3 workflow and what statuses represent to those roles............. 59

Table 8. Available Entry Statuses, their purpose, and their place within a typical workflow .................... 60

Table 9. Available Submission Statuses, their purpose, and their place within a typical workflow........... 62

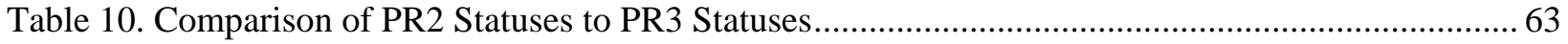

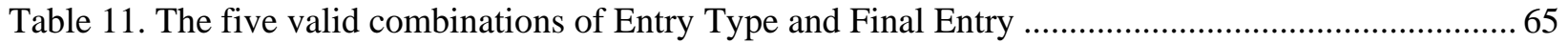

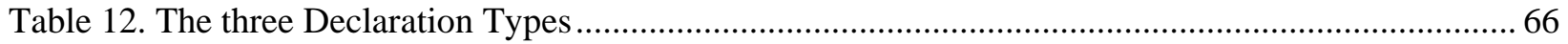





\section{ACRONYMS}

$\begin{array}{ll}\text { AP } & \text { Additional Protocol } \\ \text { DLL } & \text { Dynamic Link Library } \\ \text { DOE } & \text { US Department of Energy } \\ \text { DOM } & \text { Document Object Model } \\ \text { EIF } & \text { Entry Into Force } \\ \text { IAEA } & \text { International Atomic Energy Agency } \\ \text { ID } & \text { Identifier } \\ \text { INFCIRC } & \text { Information Circular (International Atomic Energy Agency) } \\ \text { INSEP } & \text { International Nuclear Safeguards Engagement Program } \\ \text { ISD } & \text { IAEA Safeguards Declared Information Analysis Section } \\ \text { JSON } & \text { JavaScript Object Notation } \\ \text { KMP } & \text { Key Measurement Point } \\ \text { LINQ } & \text { Language-Integrated Query } \\ \text { LOF } & \text { Location Outside Facilities } \\ \text { NNSA } & \text { National Nuclear Security Administration } \\ \text { NTLM } & \text { Network Windows Challenge/Response (authentication protocol) } \\ \text { PC } & \text { personal computer } \\ \text { PDF } & \text { Portable Document Format } \\ \text { PR1 } & \text { Protocol Reporter version 1.0 } \\ \text { PR2 } & \text { Protocol Reporter version 2.0 } \\ \text { PR3 } & \text { Protocol Reporter version 3.0 } \\ \text { R\&D } & \text { Research and Development } \\ \text { SDS } & \text { System Design Specification } \\ \text { SG } & \text { IAEA Department of Safeguards } \\ \text { SGIM } & \text { IAEA Safeguards Information Management Division } \\ \text { SQL } & \text { Structured Query Language } \\ \text { SRA } & \text { State or regional authority responsible for safeguards implementation } \\ \text { SRS } & \text { System Requirements Specification } \\ \text { SSAC } & \text { State System of Accounting for and Control for Nuclear Material } \\ \text { SSL } & \text { Secure Sockets Layer } \\ \text { UI } & \text { User Interface } \\ \text { WPF } & \text { Windows Presentation Foundation } \\ \text { XML } & \text { eXtensible Markup Language } \\ \text { XSD } & \text { XML Schema Definition } \\ & \end{array}$





\section{INTRODUCTION}

This Software Design Specification (SDS) provides design specifications for the development of the International Atomic Energy Agency (IAEA) Protocol Reporter version 3.0 (PR3) software. The specifications in this SDS are intended to support the implementation of the requirements listed in Additional Protocol (AP) Protocol Reporter Version 3.0 (PR3) System Requirements Specification (SRS), March 2014.

\subsection{PURPOSE}

The PR3 is intended to assist States in the creation, management, and submission of declarations pursuant to their obligations under the respective Additional Protocol (AP) agreements with the IAEA. It will serve as a replacement and upgrade of the Protocol Reporter version 2.0 (PR2). Therefore, its design should not only comply with the SRS but also offer improvements over the PR2 with regard to subjective measures, such as ease of use and adaptability to various declaration development and submission workflow scenarios. It should help States to develop complete and accurate declarations by providing usable forms, organized data fields, and comprehensive validation tools. It should be flexible enough to accommodate the different AP requirements for each Member State. It should also be extensible to allow for support of future AP agreements.

PR3 should offer States an efficient, flexible, and attractive alternative to other methods of developing AP declarations, thus increasing its potential for widespread adoption as the "standard" AP declaration management tool. This in turn would result in an overall improvement of the quality of AP declarations submitted to IAEA in terms of accuracy, comprehensiveness, level of detail, and consistency of format.

\subsection{TARGET USERS}

The PR3 is intended for use primarily by organizations and personnel who are involved in the performance or oversight of nuclear fuel cycle-related activities in States. The SRS defines categories into which these personnel and organizations may be divided based on their authority and necessary level of involvement in the creation, review, revision, and submission of declaration data for a State (see SRS 3.1):

- Users at the State or Regional Authority (SRA) who oversee the declaration development and submission process in their State and are authorized to submit State declarations to the IAEA

- Non-SRA users (Contributors) who provide data or information about declarable activities and locations to the SRA for potential inclusion in the State's AP declarations

This design is based on the premise that different users at either level will have varying degrees of expertise in two areas necessary to create a complete and accurate AP submission:

- Activities and locations reported in the submission

- Specific reporting obligations under the State's AP

The PR3 is designed to support both of these roles. SRA users will develop Submissions to meet AP requirements in a top-down process similar to the PR2 process. Contributor users will develop individual entries that document activities and locations, which SRA users can add to submissions.

A major objective of this design is to correlate these areas of expertise with different steps in the declaration development and submission process such that users with knowledge in either area, but not 
necessarily both, can effectively contribute to the process. When using the PR3, users will be involved in one or both of the following general steps, each of which requires different expertise (Table 1).

Table 1. Types of user involvement and knowledge required

\begin{tabular}{lcc}
\hline \multicolumn{1}{c}{ User Involvement } & $\begin{array}{c}\text { Activity / Location } \\
\text { Subject Matter Knowledge Required }\end{array}$ & $\begin{array}{c}\text { AP Knowledge } \\
\text { Required }\end{array}$ \\
\hline $\begin{array}{l}\text { Creating or Reviewing Entries } \\
\text { (SRA or Contributor level) }\end{array}$ & High & Low \\
$\begin{array}{l}\text { Assembling Entries into a } \\
\begin{array}{l}\text { Complete Submission } \\
\text { (SRA level only) }\end{array}\end{array}$ & Low & High \\
\hline
\end{tabular}

The PR3 should accommodate workflows that require only users at the SRA level who are assembling complete declarations and submissions to have detailed knowledge of the AP and the submission development process. Subject matter experts at the contributor level can focus on the technical content of the entries they are drafting for their associated sites and activities without comprehensive knowledge of the overall submission process and the AP. This allows for effective distribution of the tasks involved in the process to the parties that are most qualified and authorized to complete those tasks.

The Agency may use the PR3 for coding AP declarations that have been received on paper, but the main interaction between the Protocol Reporter and the Agency will be a State's export of declaration data into a format readable by the Agency’s systems.

\subsection{USAGE}

Because the PR3 will be used by different States with organizations and nuclear programs of varying size and complexity, it must be flexible enough to accommodate a wide range of declaration development, review, and submission workflows. These could vary from a single user at the SRA completing all declarations to a widely distributed process in which the SRA collects input from multiple users (contributors) and consolidates it for centralized review and assembly into a single submission which is then sent to the IAEA.

Fig. 1 depicts an example of a workflow model that involves:

- creating and managing individual entries at both the contributor and SRA levels,

- reviewing and assembling those entries into declarations and submissions at the SRA level,

- exporting and (optionally) transmitting declaration data for submission to the IAEA, and

- $\quad$ sending submitted data back to contributors for revision during the next declaration cycle. 


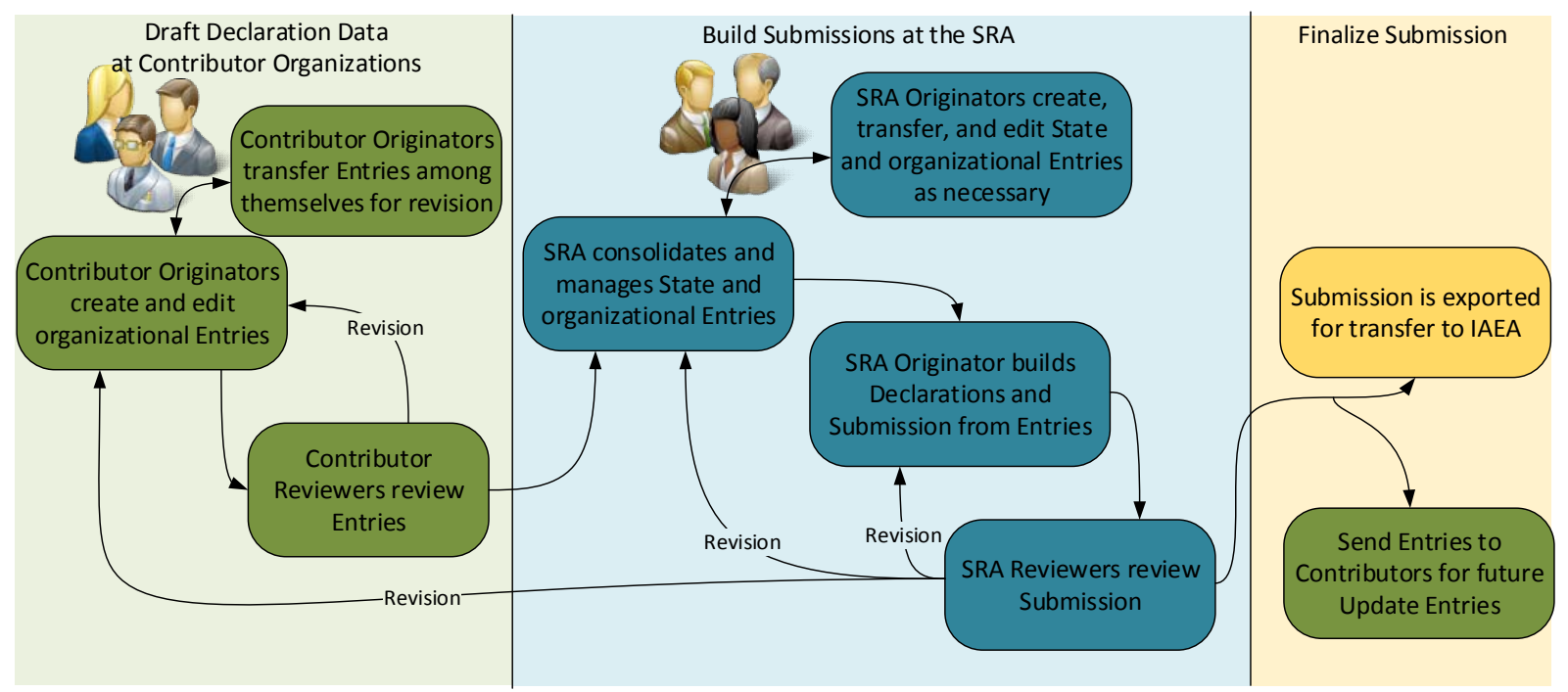

Fig. 1. Example workflow for a State with contributor organizations.

Because the Contributor level is optional, however, the PR3 can accommodate a simpler process, such as the one shown in Fig. 2.

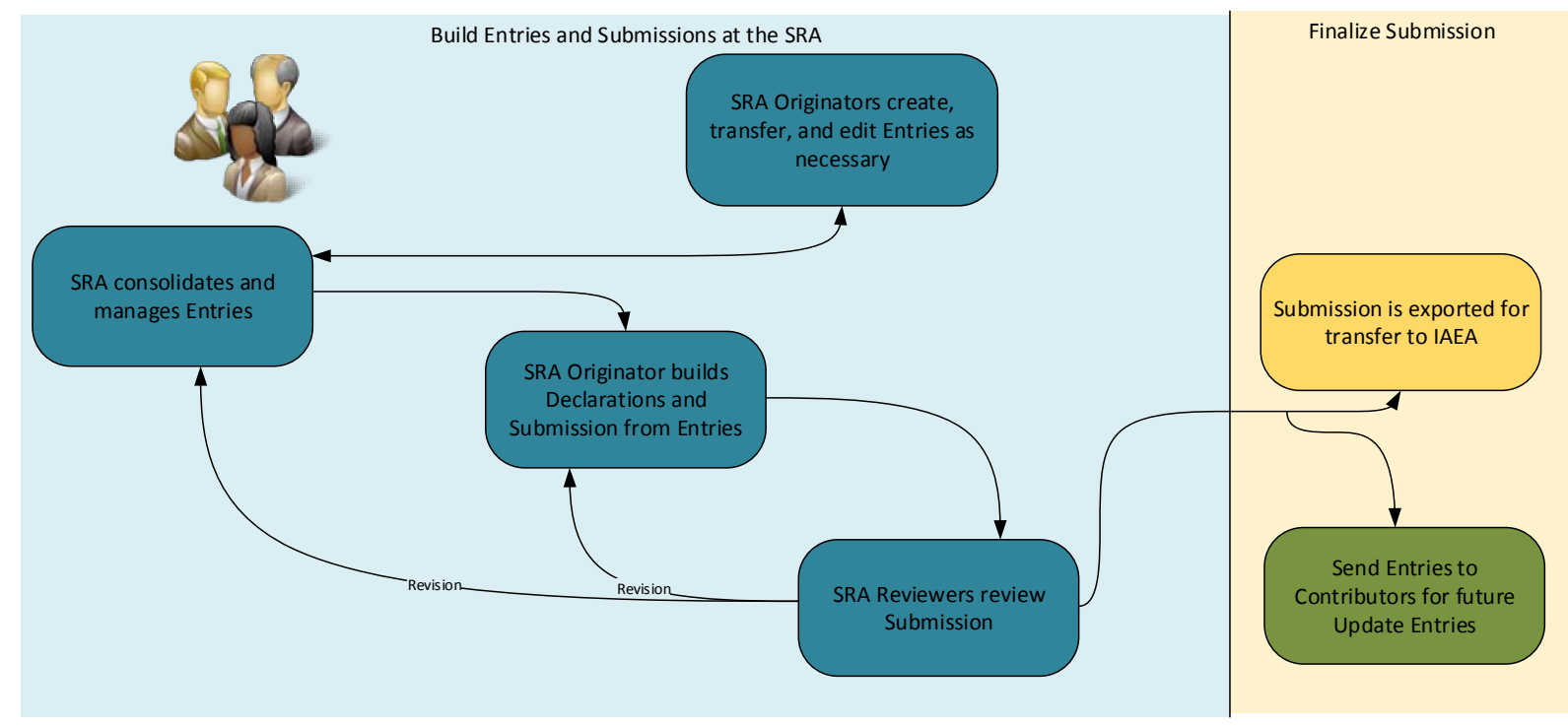

Fig. 2. Example workflow for a State without contributor organizations.

The Agency will provide the PR3 software and any applicable data templates to the SRA at each State. Each SRA is responsible for implementing policies and procedures for its distribution and use within its State. To ensure the PR3 does not constrain workflows, it does not restrict functionality according to the role of any specific user. All of its features will be available to all users and must be administratively controlled within each State as necessary.

\subsection{ENVIRONMENT}

The PR3 will run on a Windows personal computer (PC) that meets the system requirements outlined in the SRS. The PR3 will fully support 32- and 64-bit versions of Windows. The installation of software or services outside of the PR3 installer should not be required to run the PR3. 
The PR3 is a single-user application that will not require Internet or local network access to operate. Since there is no central server-side component, users will not have login accounts or specific permissions. Users may optionally share data files across a network, but SRAs and contributors must configure and manage this process.

If an optional component for electronic transmission to the Agency is included, only this feature will require Internet access and authentication.

\subsection{USER INTERFACE}

This document targets .NET Framework with Windows Presentation Foundation as the toolset used to build the PR3. Similar platforms or user interface (UI) frameworks could be used, with minor changes to design and functionality where capabilities differ.

Where possible, the UI should use basic controls and the default Windows theme and color scheme so users are presented with an interface that is already familiar to most.

While the PR3 will be developed in English, it should be developed with the ability to localize any text displayed to the user in the UI. For example, as a .NET application, any text that will be shown to the user should be retrieved from *.resx files.

In general, the UI will

- $\quad$ enable the user to organize, manage, navigate, and display large amounts of declaration data;

- provide context-specific explanations regarding the use of all forms as well as references to AP requirements; and

- $\quad$ enable users to print data or export to printable formats such as portable document format (PDF).

\subsection{INPUTS AND OUTPUTS}

As the PR3 is a standalone application, its primary source of input will be direct data entry from the user. To perform its functions, it must be able to load data template plugins and lookup files installed by the setup package or the user. For the convenience of the user, it must be able to load saved data transferred between users and exported data from previous versions of the Protocol Reporter.

The primary output from the PR3 will be a file exported for submission to the Agency. For the convenience of the user, it must be able to save declaration data and current lookup data to files that can be transferred between users. The PR3 will also produce printable or exportable reports, both to document entries and submissions for contributor and SRA users, or for submission to the Agency.

\subsection{OTHER RESOURCES}

This document outlines the selected approach, both in terms of UI and software design, for implementing the requirements as described in the SRS. The SRS provides the basic system requirements, use cases, and data structures upon which this document builds.

This document was developed in conjunction with a visual prototype of the PR3. Although the User Interface Design section (Sect. 2) covers most of the same information, the prototype helped to define look, feel, and workflow characteristics that cannot be conveyed easily through a design document. The 
prototype can also serve as a simplified introduction to the concepts covered in the SRS and SDS. See Appendix E for selected screenshots and more information about the PR3 visual prototype.

\section{USER INTERFACE DESIGN}

\subsection{USER INTERFACE STANDARDS}

This section will define the overall look and feel of the PR3 application UI. User interface controls defined in this document should be assumed to follow these standards. If a particular control is required to differ from these standards, differences will be noted in the control definition.

\subsubsection{Visual Theme}

Where possible, the PR3 UI should use standard controls and the default Windows theme and color scheme. The PR3 application should scale appropriately to the user's dots-per-inch screen settings. All text in the UI will be of a consistent typeface. When possible, the text should match the default typeface and font size used by the operating system.

In cases where the PR3 displays data that will remain internal to the State and not be submitted to the Agency, the PR3 will display this data in a way that makes it stand out from other data items in the PR3. Internal data fields will be labeled as such and will have a different background color from standard data entry fields.

In cases where a field is required for export, the PR3 will display this item in a way that makes it stand out from other data items in the PR3. Required fields will be labeled as such and will either have a different label style or a different background color from standard data entry fields.

\subsubsection{User Interface Interaction}

The UI should be navigable by both mouse and keyboard. The Tab key should cycle between interactive UI elements in a logical order, following a left-to-right, top-to-bottom order. Pressing Shift+Tab should move to the previous UI element. The selected UI element should be highlighted or surrounded by a dotted rectangle.

\subsubsection{User Interface Controls}

\subsubsection{Search Boxes}

Search boxes should have light-gray italicized text indicating the data to be searched (e.g., "Filter Entries...” instead of just "Filter"). An icon representing search should be placed to the right of the search box. Searches can be triggered either by clicking the search box or pressing the Enter key inside the text box.

When search text is entered, an " $x$ " will be displayed in the right of the text box. When the " $x$ " is clicked, the search text will be removed and the filter will be removed from the data.

Text boxes used to search and filter data should be placed at the upper right of the elements being searched. 


\subsubsection{Data Grids}

Data grids should be used whenever a list of structured data is shown to the user. Because some grids may potentially show a large number of items, tools such as filtering and sorting should be provided. This should allow users to find the particular subset of data they need to work with a consistent interface.

Wherever possible, each data grid column should be sortable and filterable. The user should be able to change the order of the columns within a data grid or the size of a particular column, and these changes should persist between sessions on the same installation of the PR3. Ideally, a standard data grid control that offers these features should be used for each data grid to ensure ease of development and consistency.

Often users will need to be able to take actions on an item or item(s) in a data grid. A user should be able to interact with items in the data grid in multiple ways:

- Toolbar buttons should be used primarily for functions that affect the entire list—adding, deleting, etc.

- Context menus should be used to take actions on a specific row or rows—editing, deleting, making bulk changes, etc.

- If the data is editable or viewable in its own form, double-clicking a row should open this form. For smaller lists with a more limited set of actions (such as sets of data within an Entry), an edit button or icon should be included in each row.

If a data grid allows the user to view or edit details of a row by linking to a separate entry form, the data grid does not need to show all fields in the item. Fields that will help the user identify or recognize the item should be shown. Complex data-type fields should be formatted to a short, readable summary (Fig. 3).

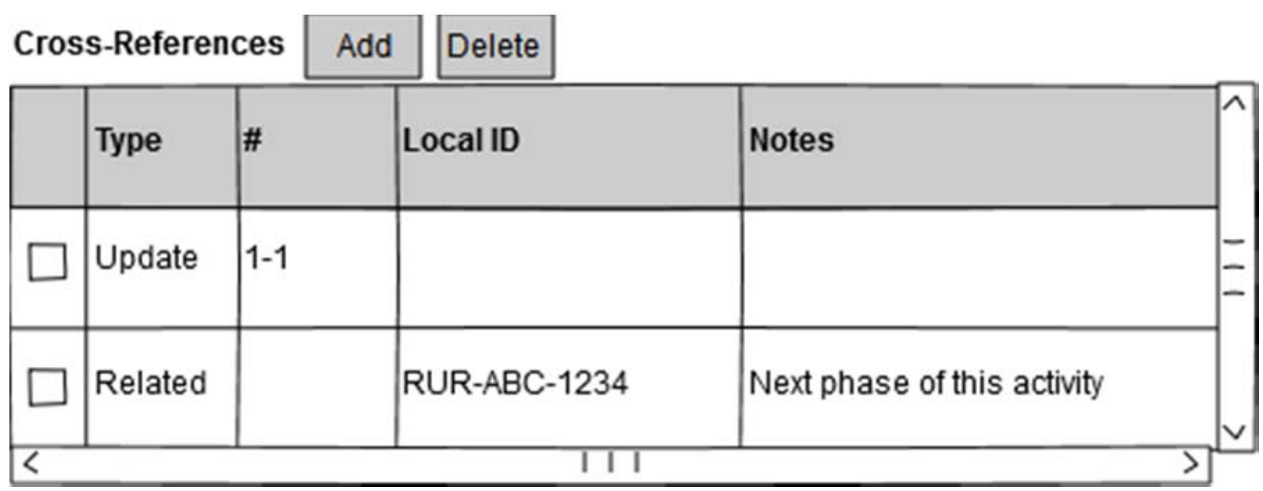

Fig. 3. An example of a data grid. This demonstrates the inclusion of toolbar icons and buttons to enable a user to take action on rows.

\subsubsection{Buttons}

Button text should be specific and reflect the action that will be taken when it is pressed. Generic terms such as "OK" and "Cancel" should be avoided (Fig. 4). 


\section{Preferred}

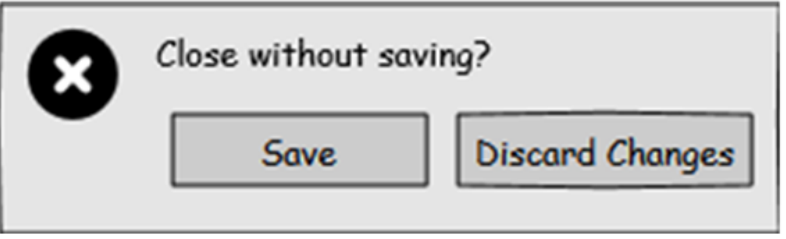

\section{Discouraged}

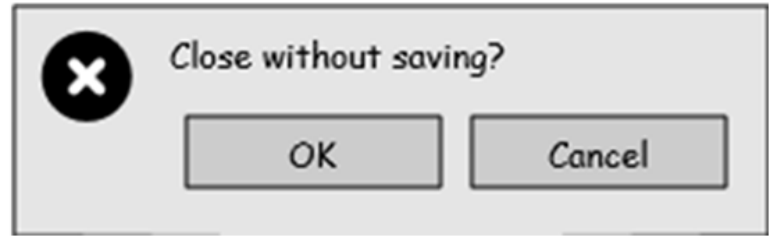

Fig. 4. An example of button labelling.

For dialog boxes, the button for the default action should be highlighted, and pressing the Enter key should trigger the click.

\subsubsection{Check Boxes}

Checkboxes should be used to collect data associated with Boolean values. Labels for checkboxes should always be displayed to the right of the checkbox. The value of the checkbox will be able to be changed both by clicking the checkbox itself as well as its label.

\subsubsection{Drop-Down Lists}

When a user is able to choose from a set of options, a drop-down list box may be used.

In cases where a field is restricted to a particular set of choices, the drop-down should be implemented as a list box, which forces the user to select one of the options. The user may not type directly into this box (though keyboard entry may be used to search for the desired value).

In cases where the options are merely suggested values, the drop-down should be implemented as a combo box. A user may type free text into a combo box, which will filter the list of options should the user choose to use the drop-down feature. However, the user is not restricted to these choices and may enter other values.

In both cases, the standard drop-down list controls for the platform or framework should be used as much as possible for consistency.

\subsubsection{Date Entry and Display}

Wherever date entry is required, a UI control that meets the following criteria should be used:

- The control must not allow the user to enter an improperly formatted or invalid date. This ensures all date fields will either remain blank or contain a valid date.

- The control should automatically convert the date value to a DateTime type (or the equivalent depending on the platform and programing language used). This standardizes date handling across the entire application and removes the responsibility for parsing dates from the PR3 data model and business rules.

- For ease of use, the control should display the date in a format consistent with the user's Windows locale. Because the date value will be stored as a DateTime object, the PR3 can reformat the date as necessary. 
- For clarity, a date format that includes a text representation of the month (e.g., "Oct” or "October" rather than " 10 ") should be used for entry and display. This prevents confusion between MM/DD and $\mathrm{DD} / \mathrm{MM}$ date formats.

- The date selection control should allow the user the option of choosing the date from a calendar rather than typing it in.

Whenever a date is displayed or saved as a text string, it should be formatted according to the following rules:

- Dates that represent declarable data should include the text representation of the month (e.g., "Oct” or "October" rather than "10") to prevent confusion between MM/DD and DD/MM formats.

- If the date is displayed in a report or exported document for use by the Agency, it should be formatted in YYYY-MM-DD format.

- If the date is exported to a human-readable format [e.g., eXtensible Markup Language (XML) or JavaScript Object Notation (JSON)] for import by the PR3 or Agency systems, it should be formatted in YYYY-MM-DD format.

\subsubsection{Toolbars}

When a number of actions can be taken on a screen, these actions should be exposed via buttons on a toolbar. Toolbars should be located directly above the elements that they are acting on or at the top of the window for more global actions. Toolbar buttons can consist of either an image and text or just an image. The most common actions that will be taken from a toolbar should have both an image and text because that will provide a larger hit-target for the user. When an image is used, it should be representative of the action taken by pressing the button. When only an image is used for a toolbar button, the tooltip for the button should describe the action taken by pressing the button.

\subsubsection{Error/Warning Messages}

Errors that prevent PR3 functionality from working as intended should not pass silently, nor should they cause the PR3 to crash. When an error occurs, a modal popup should display the error or warning and (if necessary) allow the user to choose how to respond.

Errors should also be logged in a text file that can be used for further troubleshooting.

\subsubsection{Validation Messages}

It should be easy to connect a validation message with the field that triggered it. This should either be done through visual cues (e.g., highlighting the field in red or showing an icon next to it) or behavior (e.g., setting focus on the field when the message is selected).

It should be easy to understand why a validation message was triggered. Visual cues are used to highlight a field that triggered a validation message, and details of the message should be shown near the field.

A popup should not be used to indicate that a field does not validate. In edit forms, validation should be readily available but should not divert the user's workflow. 


\subsubsection{Wizards}

Where possible, the PR3 should offer the user guided wizards or "assistants” for complex, multi-step processes or processes that require specific AP knowledge. The logic for wizards is defined in the Business Rules section (though additional wizards may be added during development).

The PR3 should maintain a consistent look and feel across wizards. The wizard is intended to ease the user into a more complex process, so the user should not have to spend time learning how each individual wizard works (

Fig. 5).

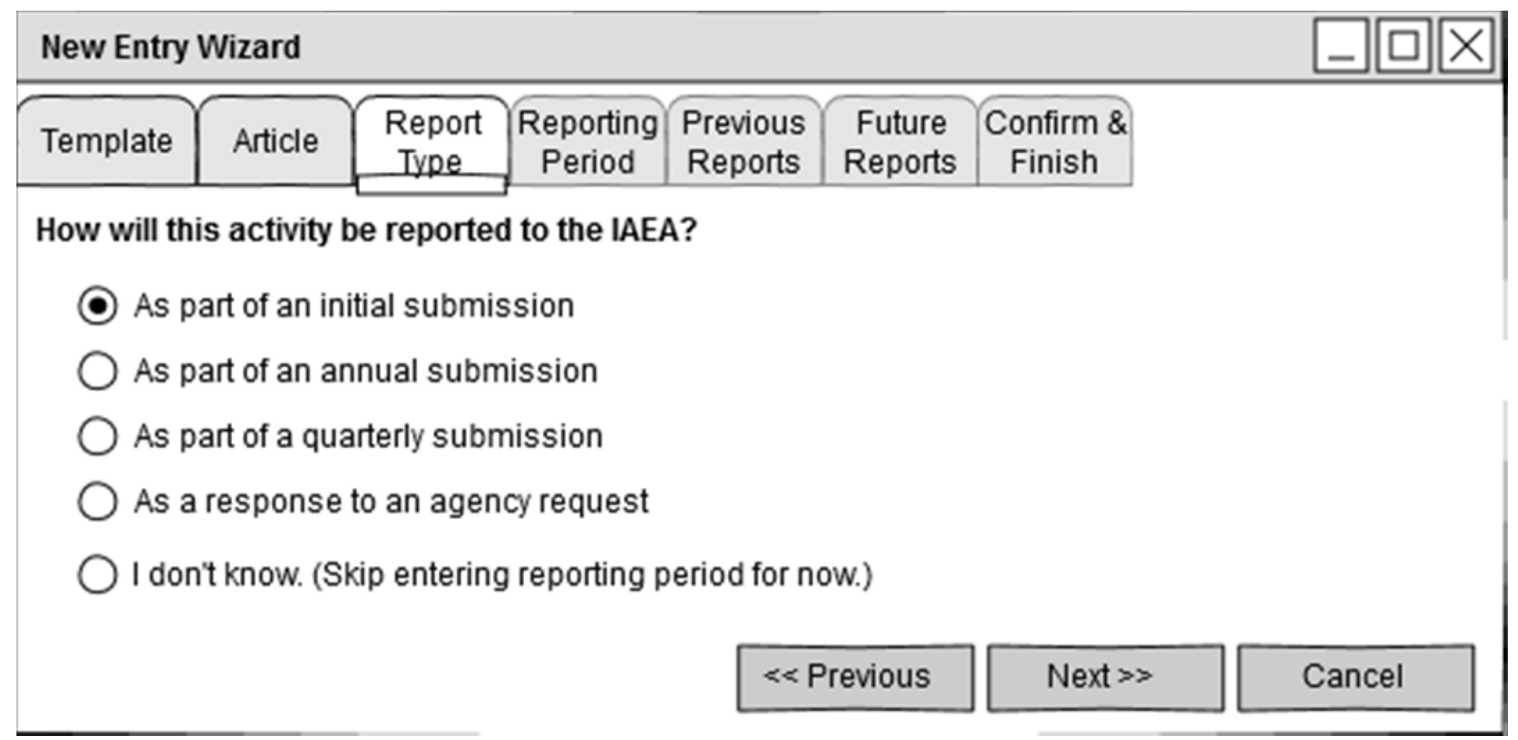

Fig. 5. An example of a wizard window.

The purpose of the data collected in each step of a wizard should be clear, and the wizard should provide the user with links to more information wherever possible. After using a wizard several times, a user should feel comfortable enough to attempt the manual process (if one is available).

To accomplish these goals, the UI for wizards should contain the following elements:

- The wizard should be a modal window so that only one wizard is open at a time, and it cannot be lost among other PR3 windows.

- A breadcrumb trail or set of tabs at the top of the wizard should indicate the user's current position in the wizard and how many steps are left.

- Next and previous buttons should allow the user to continue the process or go back and change answers.

- A user should be able to change any answer while the wizard is open although this may require them to re-answer the succeeding questions. 
- At the end of the wizard, the user should be shown a final confirmation step that summarizes all selected choices. This indicates that the wizard is complete, cleanly separating it from any windows the wizard opens.

- Where possible, wizard questions, answers, and prompts should be written descriptively rather than referencing AP-specific terms. For example, "Government-sponsored NFC R\&D not involving nuclear materials" is better than "2.a.(i)."

- If a question would provide the user with a large number of answer choices or require the user to make more than one choice, consider splitting it into two questions or allowing the user to expand/collapse groups of answers.

- Where the user is given a fixed set of options in the form of radio buttons, the first should always be selected by default.

This demonstrates the use of a breadcrumb trail or tab bar, a typical question, and navigation buttons.

\subsubsection{Reports}

The PR3 should offer reports to allow users to document their entry or submission data or other details related to the declaration development or submission process. User should be provided the ability to print reports and export them to common document formats (such as Word, PDF, or Excel).

All reports that are not valid for submission to the Agency should include the designation, "Not valid for submission to the IAEA until certified by the State.”

Where applicable, reports should offer the user the ability to provide parameters. For example, a report that calculates due dates should allow the user to change the "Entry Into Force Date" and the "As-Of Date” for calculation of the due date for an initial submission.

Reports should also allow users the ability to change the sorting and filtering of data. For example, a report showing all entries within a submission might allow a user to filter by article type. It might also allow the user to sort by Local Identifier (ID), rather than declaration and entry number.

For consistency, the same tool should be used to design and display all reports.

\subsubsection{References}

It should be possible to navigate from a cross-reference to the declaration or entry that is being referenced. This navigation should be possible even if that declaration or entry exists in a different submission so long as it exists in the user's database.

When creating a related information reference from one entry to another entry in the same submission, a reverse reference (i.e., on the second entry pointing to the first) should be created automatically.

\subsubsection{Documents}

It should be possible to save a document attached to a submission, declaration, or entry to disk or open it in its corresponding application as defined by its extension in Windows settings. 


\subsection{USER INTERFACE MODES}

Because the PR3 is meant to be used by users in an SRA as well as various contributor organizations within the State, it should be capable of operating in two modes.

The purpose of modes is to allow users to hide functionality they do not need to complete their work. Modes should be designed as a voluntary reduction in complexity, not a restriction to enforce a role.

The user should find it easy and obvious to switch modes. It should be possible to set a default mode during installation. It should also be obvious to the user which mode is currently selected and how to change it-for example, as a menu option or a drop-down list in the toolbar of the Data Manager.

\subsubsection{SRA Mode}

SRA (State or Regional Authority) mode is the standard mode of operation in the PR3 which should offer the user access to all PR3 functions. This mode is intended for users who

- have a high level of AP-specific knowledge,

- understand their State's declaration development process, and

- need to work with both submissions and entries.

\subsubsection{Contributor Mode}

Contributor mode is an optional mode of operation in the PR3. It should offer the user access to only the subset of functions relevant to contributors. This mode is intended for users who

- have little or no AP-specific knowledge,

- are subject matter experts not involved in the State declaration development process, and

- $\quad$ need to work only with entries.

Contributor mode should do the following:

- Hide the submission section in the Data Manager. (Existing submissions are still accessible if the user returns to SRA mode.)

- Hide the "New Submission” toolbar and menu commands.

- Restrict the Prepare Update Entries” wizard to creating individual entries rather than creating a submission.

- Hide or restrict any other commands or wizards used to create or edit submissions.

\subsection{USER INTERFACE WORKFLOWS}

This section defines how users move from one window to the next when performing certain tasks within the PR3.

Because the user may perform many different functions starting from the main window, this section is organized functionally using multiple diagrams.

\subsubsection{Data Management Storyboard}

The PR3 includes several different features for managing declaration data on the user's system: 
- Searching, filtering, viewing, and validating selected declaration data is done through the main Declaration Data Manager window and detail reports (Fig. 6).

- Entries and/or submissions may be gathered into transfer packages that can be sent to other users.

- Export files from the Protocol Reporter 1.0 (PR1) and Protocol Reporter 2.0 (PR2) may be viewed and saved into PR3 format.

- All of these functions are available directly from the main Data Manager window.

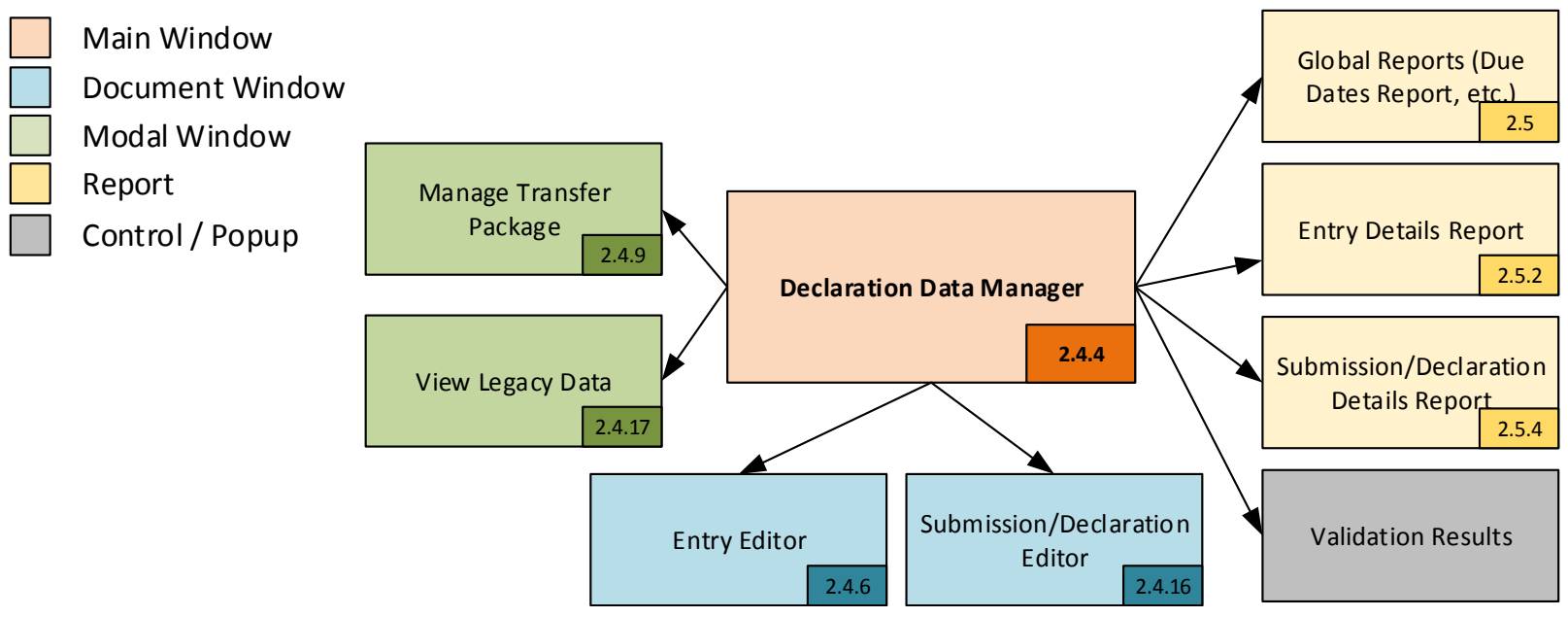

Fig. 6. The windows and interfaces used for Declaration Data Management.

\subsubsection{Entry Editing}

The PR3 supports editing Entries as independent entities. The user will create or open entries from the Declaration Data Manager window in the Entry Editor. From the Entry Editor, the user may validate the current Entry or view or print a detailed report.

Some elements of the Entry Editor will be determined by the installed data template based on the selected AP article. Entries that contain composite data types should use a common set of controls defined by the PR3 for editing those data types, including Documents, Foreign Collaboration entries, GeoCoordinates, Locations, Organizational Involvement entries, Quantities, and References (Fig. 7). 


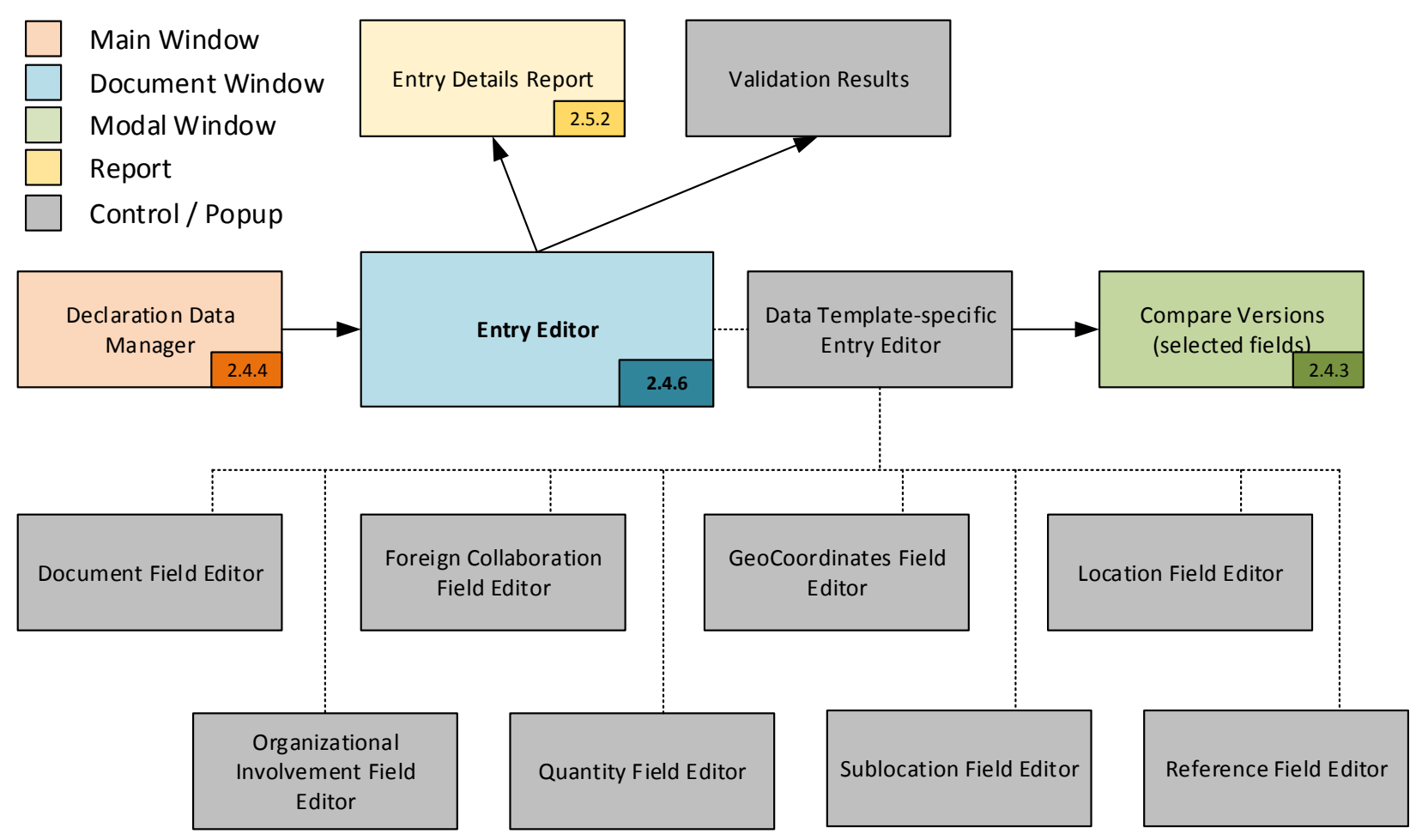

Fig. 7. The windows and interfaces used for entry editing.

\subsubsection{Submission Editing}

In the PR3, a submission and the declarations it contains are edited from the same window. The user creates or opens a submission from the Data Manager (Fig. 8). From the Submission Editor, the user may add existing entries, create or edit entries, validate the submission, or view or print a detailed report.

Some elements of the Submission/Declaration Editor will be determined by the installed data template based on the selected AP submission or declaration article. Declarations that contain composite data types should use a common set of controls defined by the PR3 for editing those data types, including documents and references. 


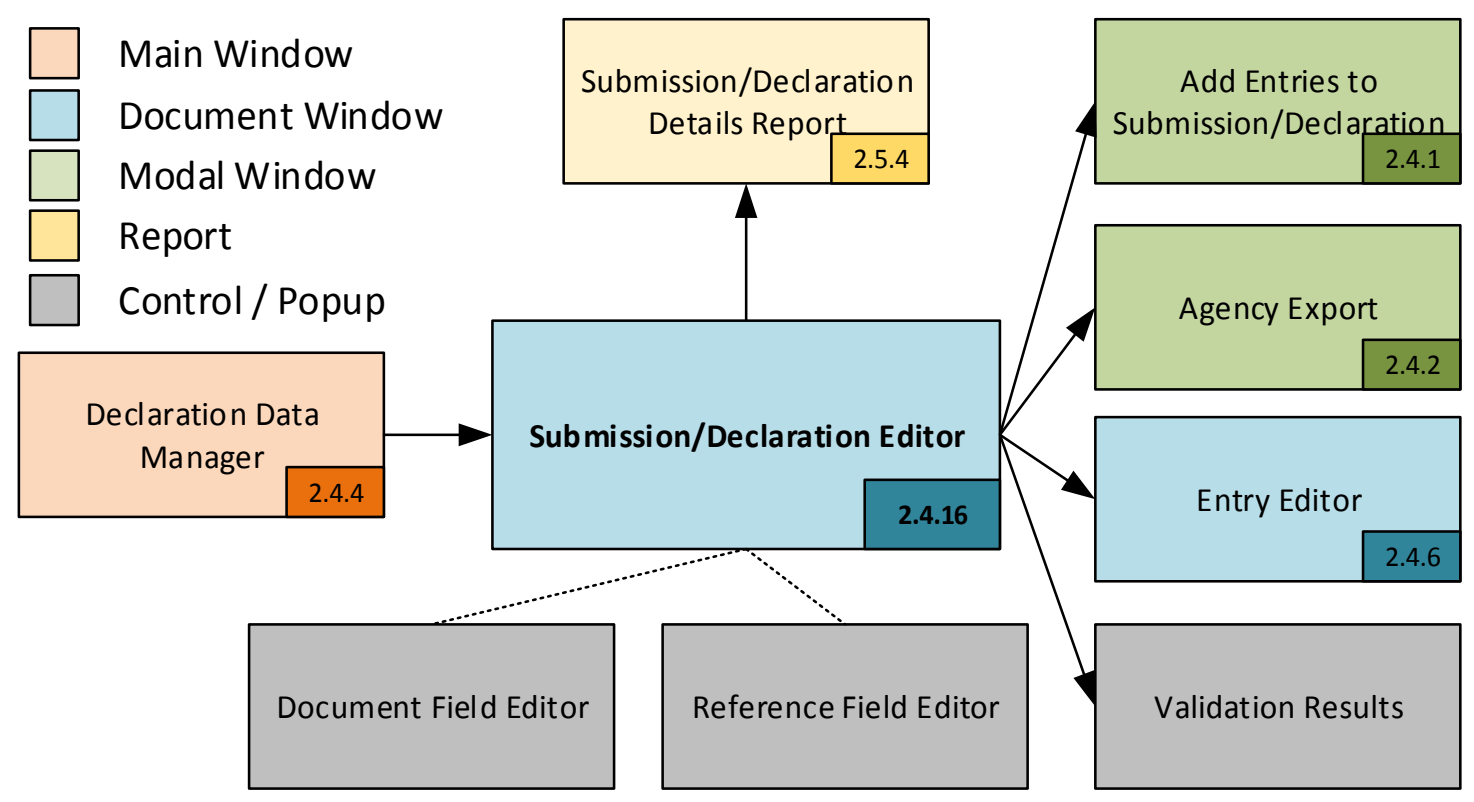

Fig. 8. The windows and interfaces used for submission editing.

\subsubsection{Application Settings}

A user may change all available configuration settings associated with the current PR3 installation through the Settings window (Fig. 9). It includes tools to:

- change general settings affecting PR3 behavior,

- record proxy server settings for network connections,

- change the locations where PR3 data is stored,

- install, remove, or update data templates,

- add, remove, or update lookup files, and

- $\quad$ edit and export State- or contributor-editable lookup data. 


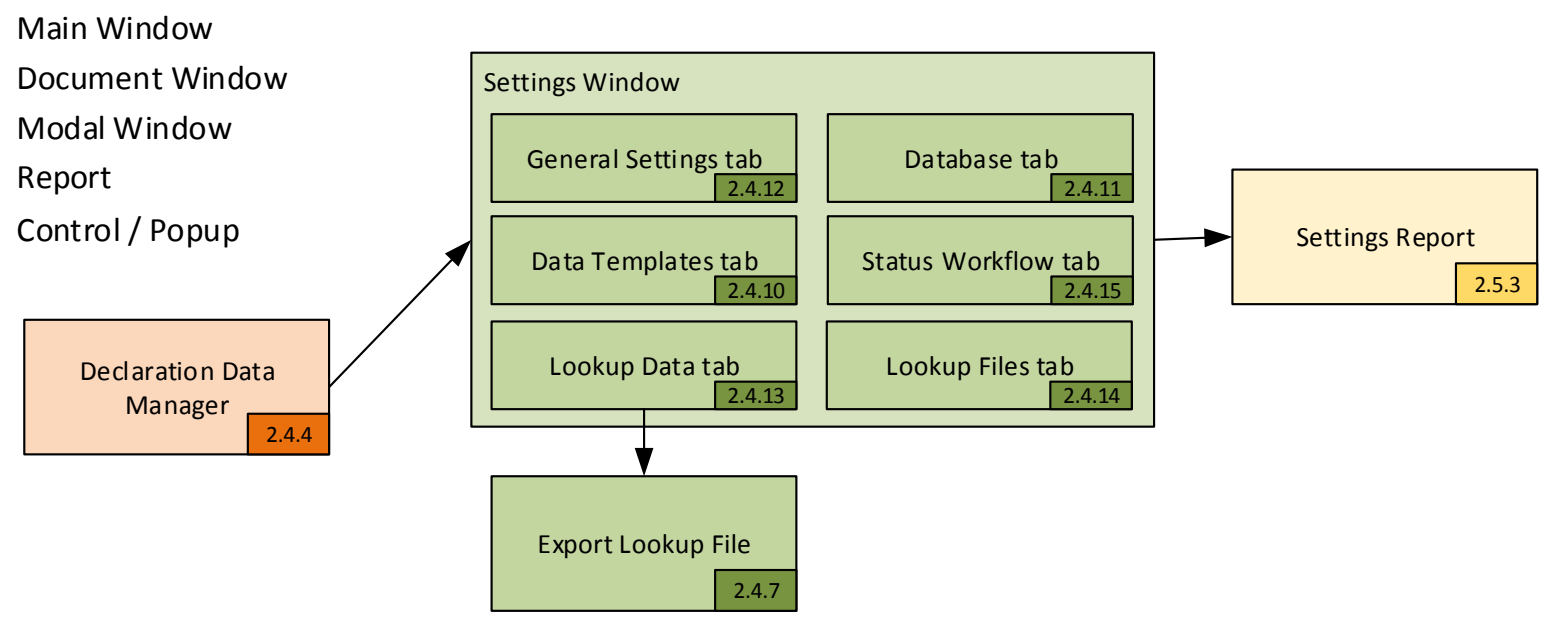

Fig. 9. The windows and interfaces used for managing settings.

\subsection{USER INTERFACE LAYOUTS}

The purpose of this section is to define the layout of each window within the PR3. Each diagram defines what data is shown on the window, how it is shown, and how the UI should respond to user input. The diagrams are intended to be simple because the actual application may deviate somewhat depending on functional or technical needs.

\subsubsection{Add Entries to Submission Window}

To streamline the process of building submissions as much as possible, the Submission Editor should offer a window that allows the user to add some or all valid entries currently indexed by the PR3.

This feature should create the necessary declarations and fill in as much data as possible. The user should only have to select the particular type of submission to be created and the entries it should contain. (Advanced users will still have the ability to create declarations and add specific entries manually.)

To help the user understand the decisions involved in this process, the window should show both eligible entries and ineligible entries from the PR3 database.

Eligible entries are those that are associated with the same data template as the submission and are valid entry articles within the submission type. Ineligible entries are either associated with a different data template or are not valid within the submission type. Both tabs are shown so that the user can account for all entries in the PR3 database and understand why an entry may not be eligible for selection.

Eligible entries will show the Title, Local ID, Article, and Contributor for each indexed entry that can be added to the current submission (based on Article, Data Template, and Status). The user may check or uncheck each entry, filtering and sorting the grid as necessary. The user should also be able to open entries directly from this list to view more details (Fig. 10). 


\begin{tabular}{|l|l|l|l|l|}
\hline \multicolumn{2}{|l|}{ Add Entries to Submission } & \multicolumn{1}{|l|}{ Ineligible Entries (10) } \\
\hline \hline Eligible Entries (25) & \multicolumn{1}{|l|}{ Local ID } & Article & Contributor \\
\hline \hline & Title & RA-01-01 & 2.a.(i) & RAPA \\
\hline$\square$ & RAPA Isotope Separation & RA-02-02 & 2.a.(iii) & SRA \\
\hline$\square$ & $\begin{array}{l}\text { Fuel fabrication and testing } \\
\text { facility }\end{array}$ & RA-03-03 & 2.a.(iv) & SRA \\
\hline$\square$ & Heavy water production & Add Selected Entries \\
\hline
\end{tabular}

Fig. 10. The Eligible Entries tab in the Add Entries to Submission window.

Ineligible entries will show the Title, Local ID, Article, Data Template, Contributor, and a reason why the entry is considered ineligible. Users cannot select these entries. This tab is included so users can understand why some entries are not available for selection.

Note: Entries that are ineligible because of their associated Article or Data Template cannot be made "eligible" through any sort of editing. Article and Data Template are set when entries are created (Fig. 11).

\begin{tabular}{|l|l|l|l|l|l|}
\hline \multicolumn{2}{|l|}{ Add Entries to Submission } & & Reason \\
\hline \multicolumn{1}{|l|}{ Eligible Entries (25) } & Ineligible Entries (10) & & Contributor \\
\hline Title & Local ID & Article & Template & The article type is not valid for this type of \\
\hline $\begin{array}{l}\text { RAPA Isotope } \\
\text { Separation }\end{array}$ & RA-01-01 & 2.a.(i) & Ruritania & RAPA \\
\hline $\begin{array}{l}\text { Fuel fabrication and } \\
\text { testing facility }\end{array}$ & RA-02-02 & 2.a.(iii) & EU & $\begin{array}{l}\text { The data template is not valid for this } \\
\text { submission. }\end{array}$ & SRA \\
\hline
\end{tabular}

Fig. 11. The "Ineligible Entries" tab in the “Add Entries to Submission” window.

\subsubsection{Agency Export Window}

When the user chooses to export a Submission for transmission to the Agency, this window serves as a final confirmation (Fig. 12). It presents the user with a current list of validation messages (errors, warnings, and information, which can be filtered) and an explanation of what these validation messages mean. 


\begin{tabular}{|c|c|c|c|c|c|c|c|}
\hline \multicolumn{6}{|c|}{ Agency Export } & -[ & $\square$ \\
\hline \multicolumn{8}{|c|}{ The following validation issues were found in this Submission. } \\
\hline \multicolumn{8}{|c|}{$\begin{array}{l}\text { Error messages typically reflect major problems with your Submission. You may not transmit a } \\
\text { Submission with errors. }\end{array}$} \\
\hline \multicolumn{2}{|c|}{ Errors (1) } & Warnings (1) & Info (1) & & & & \\
\hline Category & \multicolumn{3}{|c|}{ Message } & \multicolumn{4}{|l|}{ Location } \\
\hline Info & \multicolumn{3}{|c|}{ No Project Title was entered } & \multicolumn{4}{|l|}{ Entry 1-1 } \\
\hline Warning & \multicolumn{3}{|c|}{ No Local ID was entered } & \multicolumn{4}{|l|}{ Entry $1-1$} \\
\hline Error & \multicolumn{3}{|c|}{ No Fuel Cycle Stages were selected } & \multicolumn{4}{|l|}{ Entry 1-2 } \\
\hline \multicolumn{5}{|c|}{ C:IUsersIMyUsernamelDocumentsMy PR3 FilesISubmission-3a- } & Browse... & Export & \\
\hline
\end{tabular}

Fig. 12. The Agency Export window.

If there are any errors in the validation messages list, then the export button should be disabled. Warnings and informational messages will not prevent a user from exporting a Submission, but the user should understand the implications of submitting data with warnings.

The user may choose where to export the Submission and then click the Export button to complete the process. This should set the selected Submission and its Entries to "Submitted" status.

If the PR3 is to support electronic transmission to the Agency, then that option will be made available here.

\subsubsection{Compare Versions Window}

If an Entry or Submission updates previous data and that data is available (either referenced or stored as part of the new Update Entry or Submission), then the PR3 should allow the user to compare multi-line text fields to their previous values (Fig. 13). 


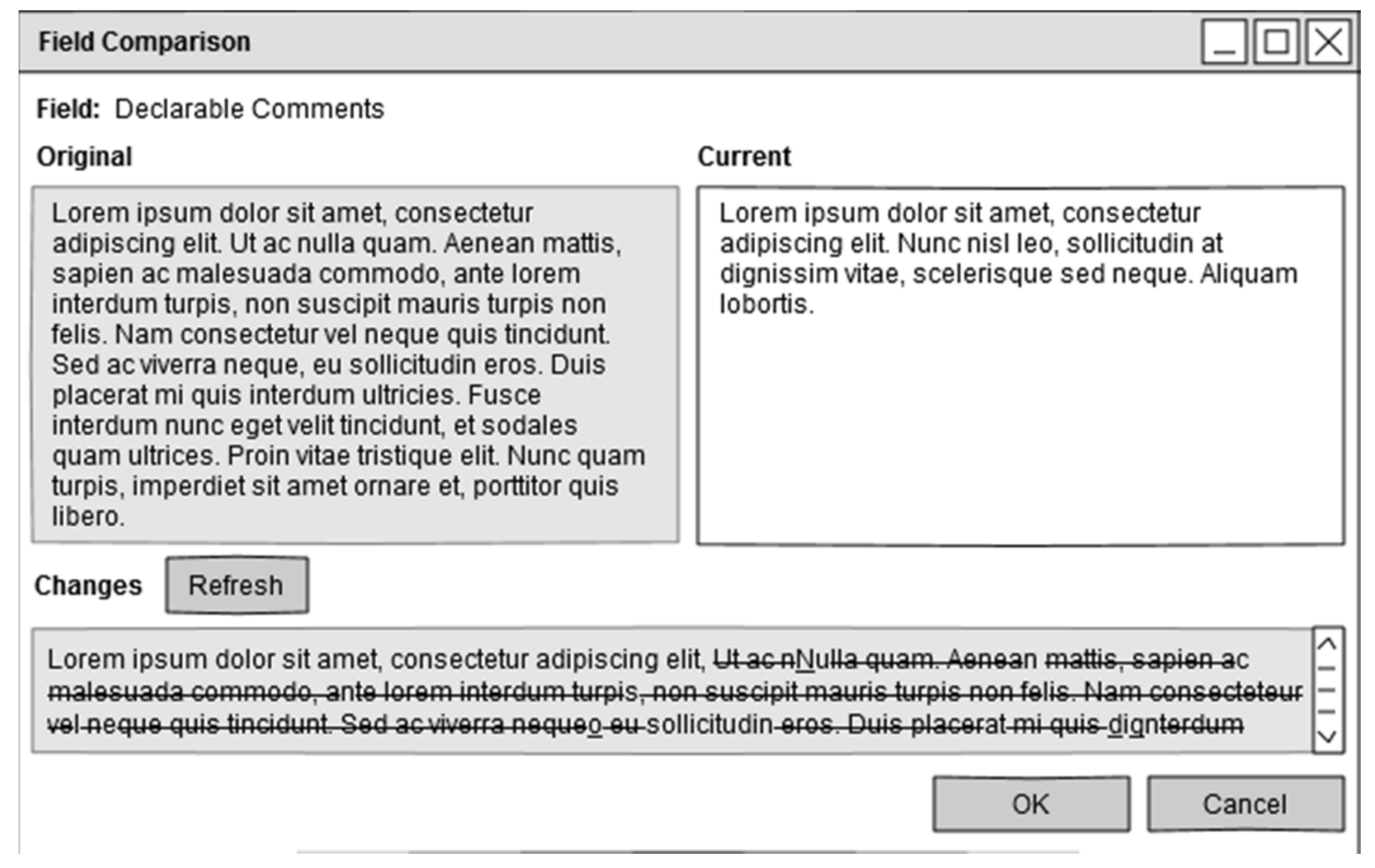

Fig. 13. The Compare Versions window.

The version comparison should show the user a formatted representation of the differences between the original and current field values. (This is similar to Microsoft Word's track changes features or the "diff" tools included in many source control tools.) This should help identify which sections have been added, modified, or removed.

Note the version comparison feature is only intended to be used for multi-line text fields. Since a user can open multiple Entries and Submissions, making comparisons between simpler fields can be done by switching between windows.

\subsubsection{Declaration Data Manager Window}

The Data Manager window is the main hub for navigation within the PR3 (Fig. 14). 


\begin{tabular}{|c|c|c|c|c|c|c|}
\hline \multicolumn{6}{|c|}{ Data Manager } & \multirow[t]{2}{*}{$-\square$} \\
\hline + Entry & + Submission & & \multicolumn{3}{|c|}{ Search } & \\
\hline \multirow{2}{*}{\multicolumn{2}{|c|}{$\begin{array}{l}\text { Entries } \\
\text { All Entries } \\
\text { Completed Entries } \\
\text { In-Progress Entries }\end{array}$}} & \multicolumn{2}{|l|}{ Title } & Article & Status & Template \\
\hline & & \multicolumn{2}{|l|}{ Heavy water production } & 2.a.(iv) & In Progress & Model AP \\
\hline \multirow{3}{*}{\multicolumn{2}{|c|}{$\begin{array}{l}\text { Submissions } \\
\text { All Submissions } \\
\text { Completed Submissions } \\
\text { In-Progress Submissions } \\
\text { Submitted Submissions }\end{array}$}} & \multicolumn{2}{|c|}{ Import of PWR control rods from Idustralia } & 2.a.(ix)(b) & In Progress & Ruritania \\
\hline & & \multicolumn{2}{|l|}{$\begin{array}{l}\text { Ten year plan: Multi-unit nuclear power } \\
\text { station }\end{array}$} & 2.a.(x) & In Progress & Model AP \\
\hline & & \multicolumn{2}{|l|}{ Additional Documents } & Note & In Progress & Model AP \\
\hline & & \multicolumn{5}{|c|}{$\begin{array}{l}\text { Selected Item Summary } \\
\text { Title: Heavy water production } \\
\text { Article: } 2 \text {.a.(iv) } \\
\text { Status: In Progress } \\
\text { Template: Model AP } \\
\text { Reporting Period: } 2013-01-01 \text { to 2013-12-31 }\end{array}$} \\
\hline
\end{tabular}

Fig. 14. The Declaration Data Manager window.

It allows users to browse, organize, and edit any Entries and Submissions on their computer in two ways:

- Browsing data by pre-set filters: The user should be able to browse all Entries and Submissions stored in the PR3 (left pane). This window should allow the user to select filtered views of the data. A variety of standard filters should be available (including, but not limited to, status). Users should be able to define their own filters for this view.

- Searching: The user should be able to perform a search across all indexed data. If a filter is selected, then the search should be performed within that filtered subset of the data.

The content of the item list in the right pane should change based on the search term and/or the selection in the left panel. Users should be able to use context menus to edit, view, validate, copy, change status, or delete Entries or Submissions in the left pane. (When the user takes an action that cannot be undone, such as deletion or bulk status change, a confirmation prompt should be shown.) A summary of the data in a selected item should be shown at the bottom of the right panel.

\subsubsection{Entry Editor Window}

When editing Entries (whether individually or within a Submission), a user will use the Entry Editor window (Fig. 15). 


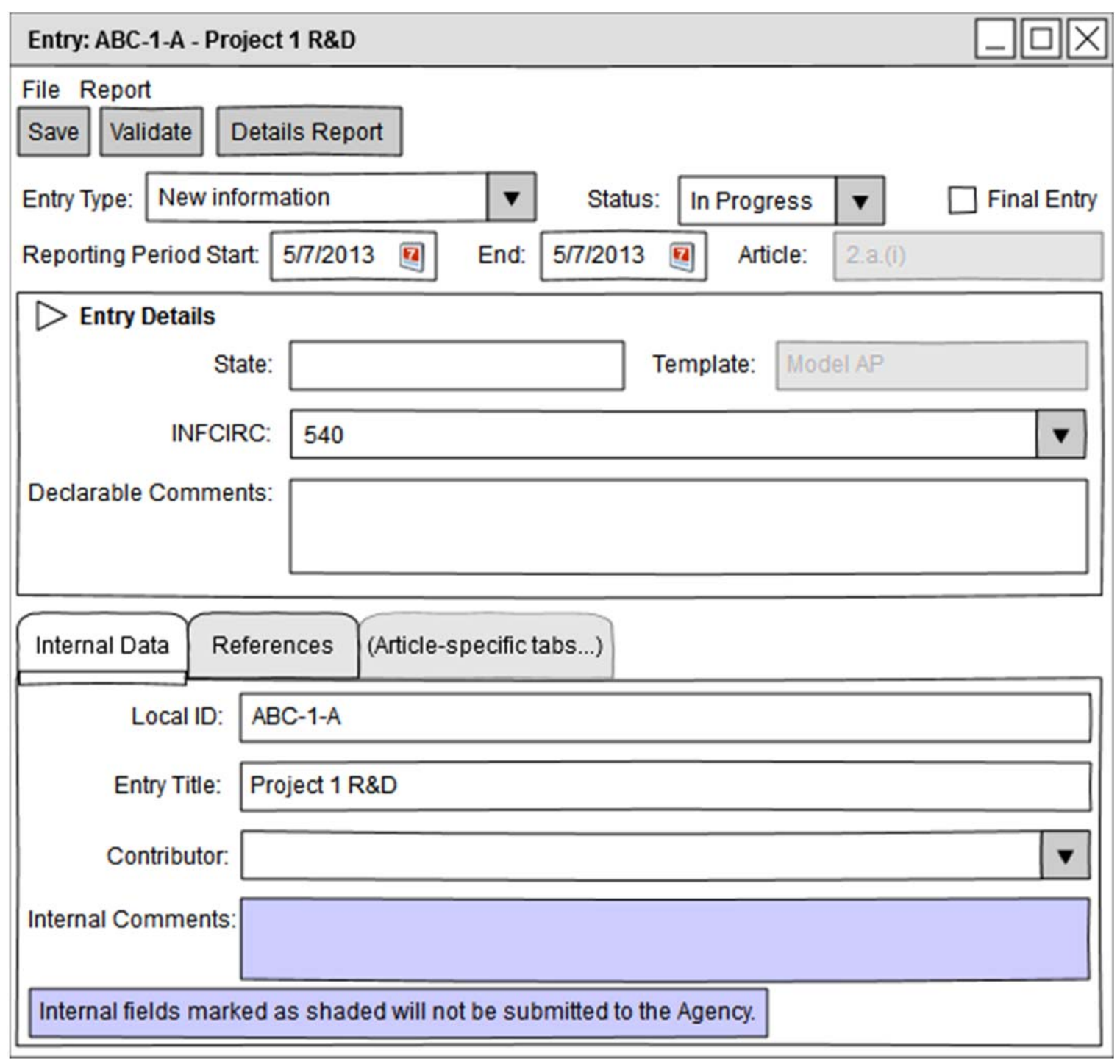

Fig. 15. The Entry Editor window, with the Internal Data tab selected.

The layout of the Entry Editor should clearly communicate the following:

- The separation of internal data used by the State from declarable data that is sent to the Agency

- The separation of "header" fields used for identification (which will likely be unnecessary once the Entry is added to a Declaration) from "detail” fields, which are filled in to meet AP requirements

- How to save, validate, and print the Entry at any time

The design should prevent the breadth and granularity of the data collected by the PR3 from overwhelming the user. Wherever possible, the Entry Editor should make use of UI elements such as expanders and tabs to highlight the most commonly used fields while making it clear how to find other fields as necessary.

Because the PR3 must support multiple Entry types based on multiple data templates, the Entry Editor window must be a mix of fixed and dynamically loaded elements. The diagrams in this section show the 
fixed elements that will be constant for all Entries (Fig. 15, Fig. 16). Dynamic elements will be loaded from data template plugins and are defined in the Components section.

To accommodate this variability, the Entry Editor should have a common location and format for loading all dynamic controls. In these diagrams, a tab strip provides a way to expand the form in a consistent manner. A template would provide sets of controls for template-specific data elements by returning collections of tab controls. This also simplifies layout for Entries with numerous fields; data templates can group the fields in multiple tabs rather than trying to fit them all in one panel.

For sets of multi-column data (such as References, Documents, or Locations), the Entry Editor form should provide a grid that allows the user to add, edit, or remove items. Wherever possible, the Entry Editor and any forms provided by data templates should use common controls for composite data types for consistency and ease of development.

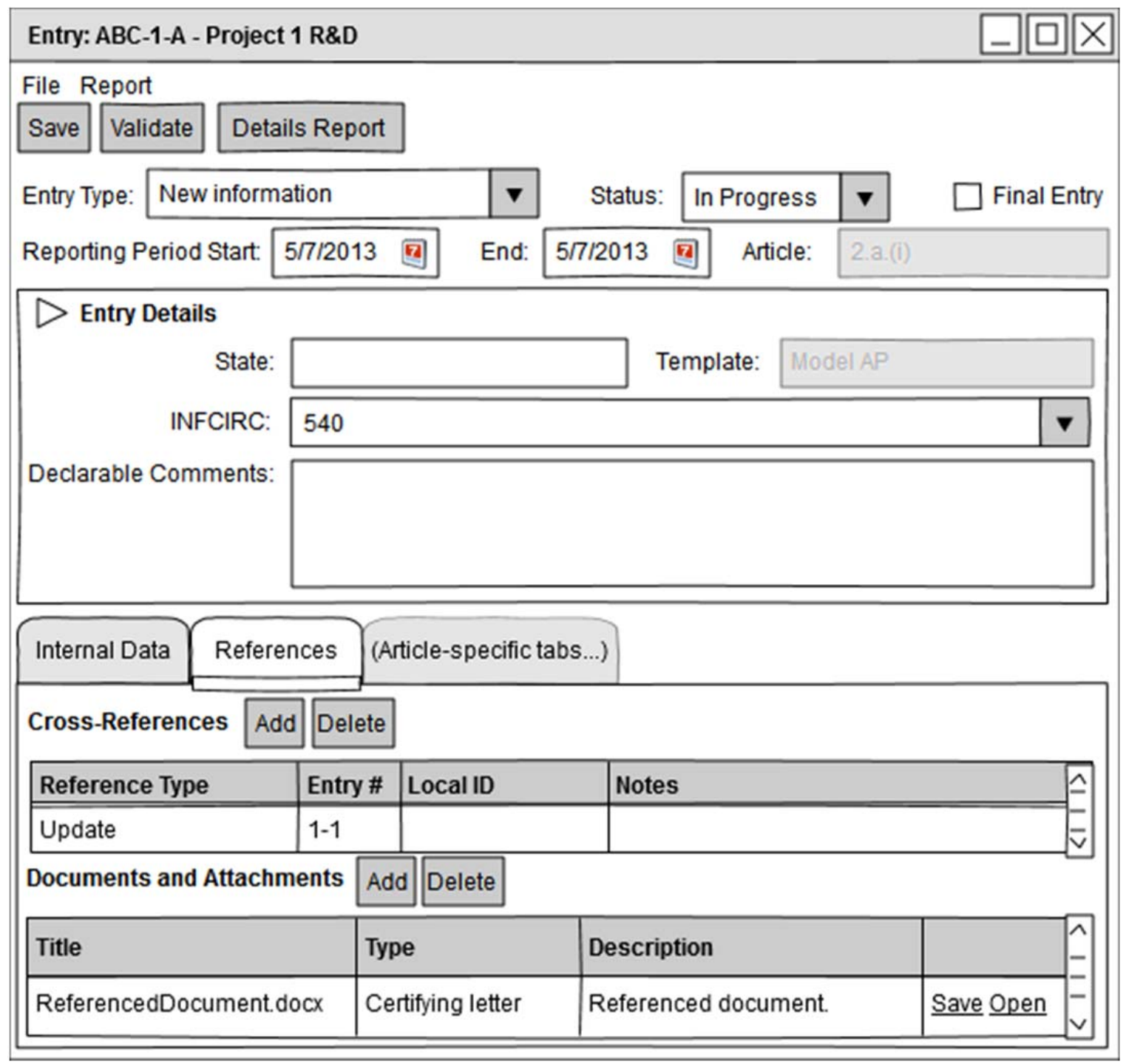

Fig. 16. The Entry Editor window, with the References tab selected. 


\subsubsection{Export Lookup File Window}

A user may export any State-editable lookup data to a lookup file that can be imported into other installations of the PR3 (Fig. 17). This allows users to distribute lookup data to other users.

\begin{tabular}{|c|c|c|c|c|c|c|c|}
\hline Export Lookup File & & & & & & & $-\square[$ \\
\hline \multirow{5}{*}{$\begin{array}{l}\text { Contributor } \\
\text { Facility/LOF } \\
\text { Location } \\
\text { MBA Code } \\
\text { Mine/Plant } \\
\text { Site Code }\end{array}$} & $\wedge$ & $\square$ & Building & Room & Subarea & Facility/LOF & $\begin{array}{l}\text { Managed } \\
\text { Access }\end{array}$ \\
\hline & & $\square$ & RBE & & & RBE- & $\square$ \\
\hline & $E$ & $\square$ & RBF & & & RBF- & $\square$ \\
\hline & & $\square$ & RBR & & & RBR- & $\square$ \\
\hline & 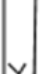 & $\square$ & RA-1 & & & RBF- & $\square$ \\
\hline & & & & & & Export... & Cancel \\
\hline
\end{tabular}

Fig. 17. The Export Lookup File window.

When exporting a file, a user may select any or all lookup items in the PR3 installation whether they came from standard lookup files or were individually added by the user.

\subsubsection{Load Transfer Package Window}

When opening a Transfer Package for import, the user should see a different set of buttons than for export in the Transfer Package window (Fig. 18).

\begin{tabular}{|c|c|c|c|c|c|c|c|}
\hline \multicolumn{7}{|c|}{ Transfer Package: Transfer1.pr3t } & $-\square$ \\
\hline Save selected files & Save all files & $\square$ or & nport, ch & ange status of & Il items to: & SRARevie & \begin{tabular}{l|l}
$\mathrm{w}$ & $\boldsymbol{\nabla}$
\end{tabular} \\
\hline \multicolumn{2}{|l|}{ Title } & \begin{tabular}{|l} 
File \\
Type
\end{tabular} & Article & Status & Template & Modified & Filename \\
\hline \multicolumn{2}{|c|}{ RAPA Isotope Separation - Phase I } & Entry & 2.a.(i) & In Progress & Ruritania & $\begin{array}{l}7 / 7 / 2014 \\
2: 00 \mathrm{pm}\end{array}$ & file50.pr3e \\
\hline \multicolumn{2}{|c|}{ RAPA Isotope Separation - Phase II } & Entry & 2.a.(i) & In Progress & Ruritania & $\begin{array}{l}7 / 7 / 2014 \\
2: 00 \mathrm{pm}\end{array}$ & file51.pr3e \\
\hline
\end{tabular}

Fig. 18. The Load Transfer Package window.

A user should be able to save either the entire contents of the Transfer Package to the local computer, or he/she should be able to save selected entries or submissions. The user should have the option to automatically set the imported item's Status in case the sender set the Status incorrectly. 


\subsubsection{Manage Transfer Package Window}

The Transfer Package functionality in PR3 is intended to support transferring large numbers of Entries and/or Submissions between users. Ideally, Transfer Packages should work much like the zipped folders functionality in Windows, allowing multiple files to be saved and transferred as a single unit.

When the user creates a Transfer Package, a list of selected items will appear in a new window (Fig. 19). A user may add items to or remove items from a Transfer Package as necessary. When finished, the user may save it to a specified file location.

\begin{tabular}{|l|l|l|l|l|l|l|}
\hline \multicolumn{1}{|l|}{ Transfer Package: Untitled.pr3t } \\
\hline \hline Save Add files \\
\hline Title \\
\hline
\end{tabular}

Fig. 19. The Manage Transfer Package window.

The user should also have the option to set the status of all items in the Transfer Package upon save. This function should affect both the items stored in the Transfer Package as well as the original items that were selected (if they are still available on the user's system). This would allow for scenarios such as the following:

- A Contributor Editor is finished with an initial draft and needs to send multiple Entries to a Contributor or SRA Reviewer. The Entries are currently marked as "In Progress" but should be set to "Review" status upon export.

- A Contributor or SRA Reviewer has determined that Entries need to be sent back to the Contributor Editor for further revision. The Entries are currently marked as "Review" but should be set to "Revision Required" status upon export.

\subsubsection{Settings: Data Templates Tab}

While most users will only have the pre-installed template used by their respective States, the Settings window should allow the possibility for multiple data templates to be installed because there are cases where it may be necessary (Fig. 20). In such cases, one data template will be chosen as the default for creating new Entries. 


\begin{tabular}{|c|c|c|c|c|c|c|}
\hline \multicolumn{6}{|l|}{ Settings } & 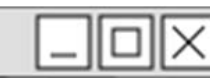 \\
\hline General & Database & $\begin{array}{c}\text { Status } \\
\text { Workflow }\end{array}$ & Network & Templates & Lookup Files & Lookup Data \\
\hline \multicolumn{2}{|l|}{ Name } & \multicolumn{3}{|l|}{ Filename } & Version & Add... \\
\hline \multicolumn{2}{|l|}{ Ruritania } & \multicolumn{3}{|c|}{ IAEA.ProtocoIReporter.Ruritania.dll } & 3.0 & Remove \\
\hline \multicolumn{2}{|l|}{ Model AP } & \multicolumn{3}{|c|}{ IAEA.ProtocolReporter.ModelAP.dll } & 3.1 & Set Default \\
\hline & & View & ettings Rep & & Save & Cancel \\
\hline
\end{tabular}

Fig. 20. The Data Templates tab of the Settings window.

\subsubsection{Settings: Database Tab}

A database will be created for each instance of the PR3 upon its first startup. This process should be mostly transparent to the user. However, some users may wish to create another empty database or switch databases. These controls should be made available in Settings (Fig. 21).

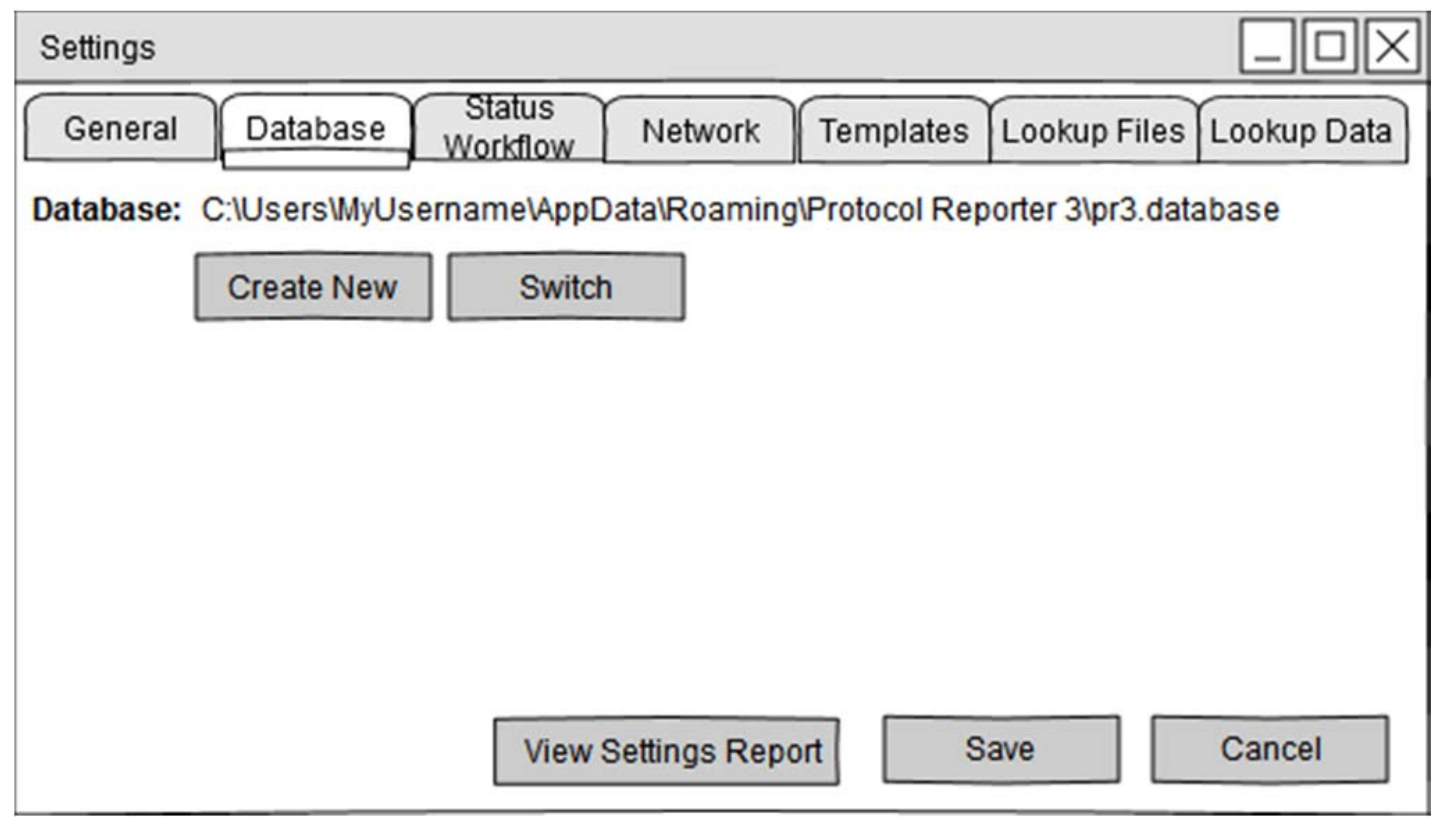

Fig. 21. The Database tab of the Settings window.

In addition, if any functionality is added to import, export, backup, or restore a database as a whole, it should be found here. 


\subsubsection{Settings: General Settings Tab}

The General Settings tab (Fig. 22) is used to change any settings that affect the workflow of the PR3 but are not related to either external data or technical functionality. For more information, see Settings Definition in Components (Sect. 3.8.2).

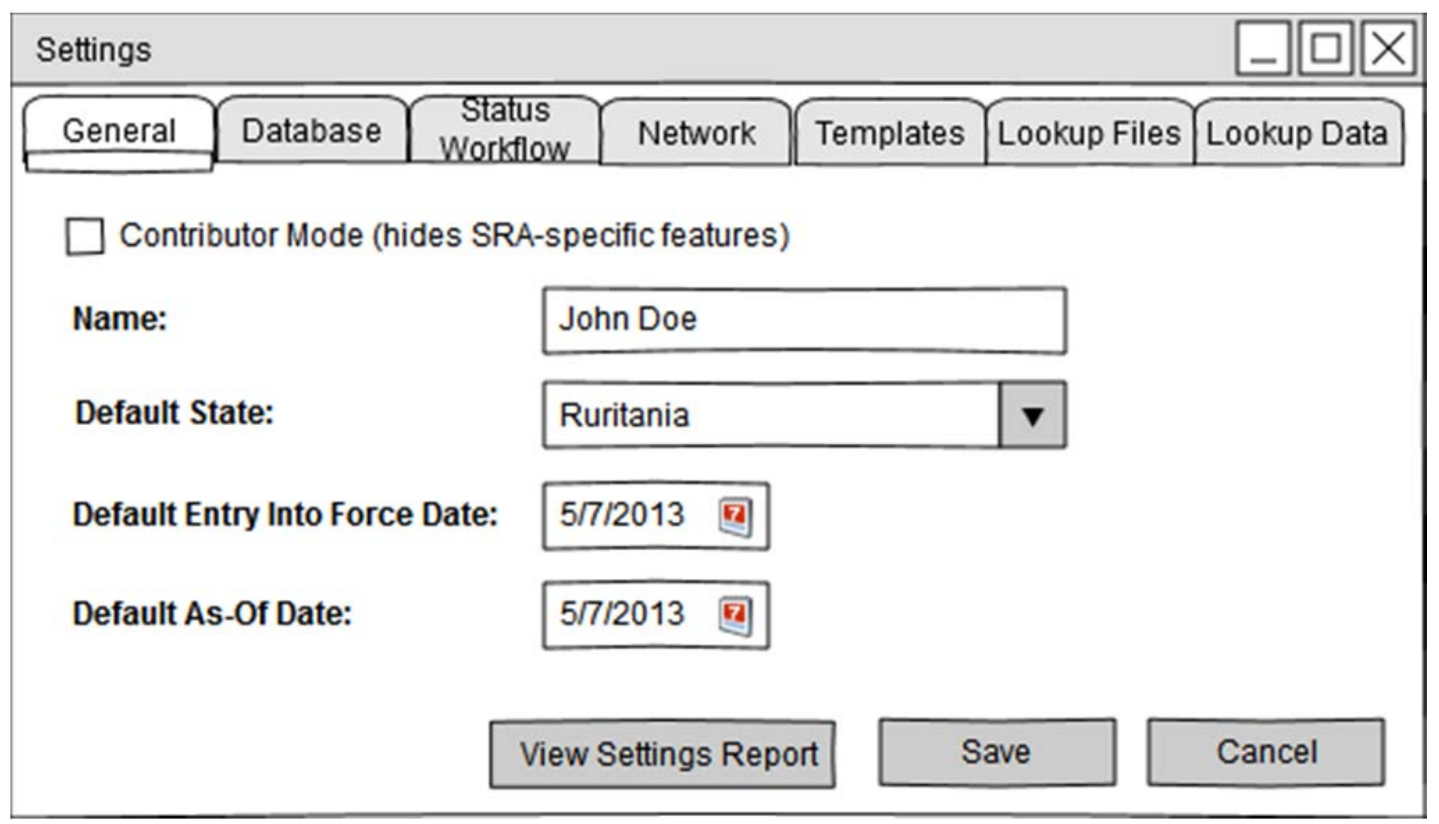

Fig. 22. The General tab of the Settings window.

\subsubsection{Settings: Lookup Data Tab}

The Lookup Data tab (Fig. 23. The Lookup Data tab of the Settings window.) is used to edit all Stateprovided lookup lists (see SRS C.1). The user may create, edit, and delete new entries for these lists. Lookup lists that are provided by the Agency may not be modified. The user may also export a new lookup file based on some or all of the lookup data stored in his/her PR3 installation. 


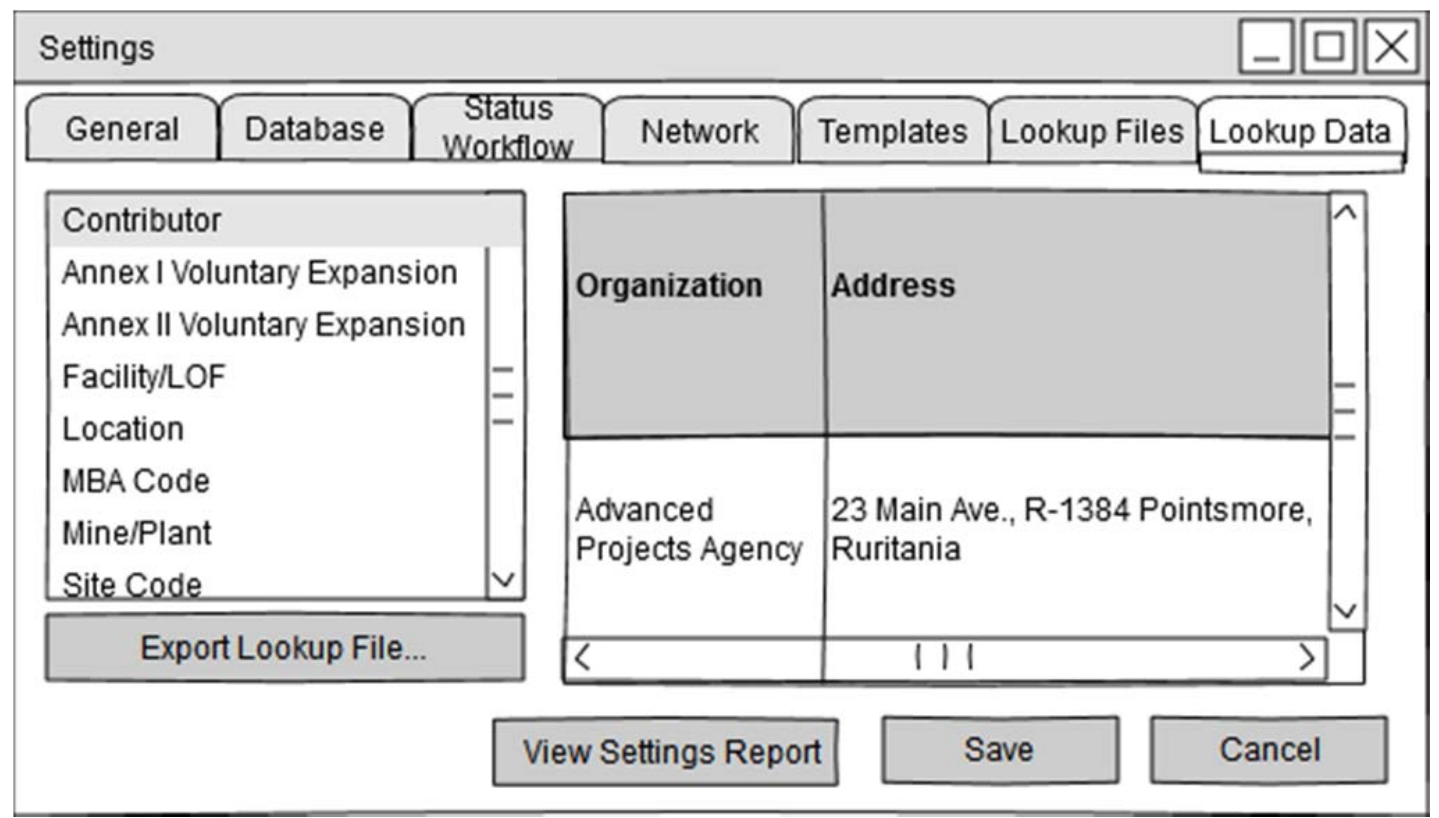

Fig. 23. The Lookup Data tab of the Settings window.

\subsubsection{Settings: Lookup Files Tab}

Lookup data may be loaded from multiple files provided by the Agency, the Member State's SRA, or other Contributors (e.g., all users at the same Site may use the same set of Locations). This tab provides an interface to add, update, and prioritize these files (Fig. 24).

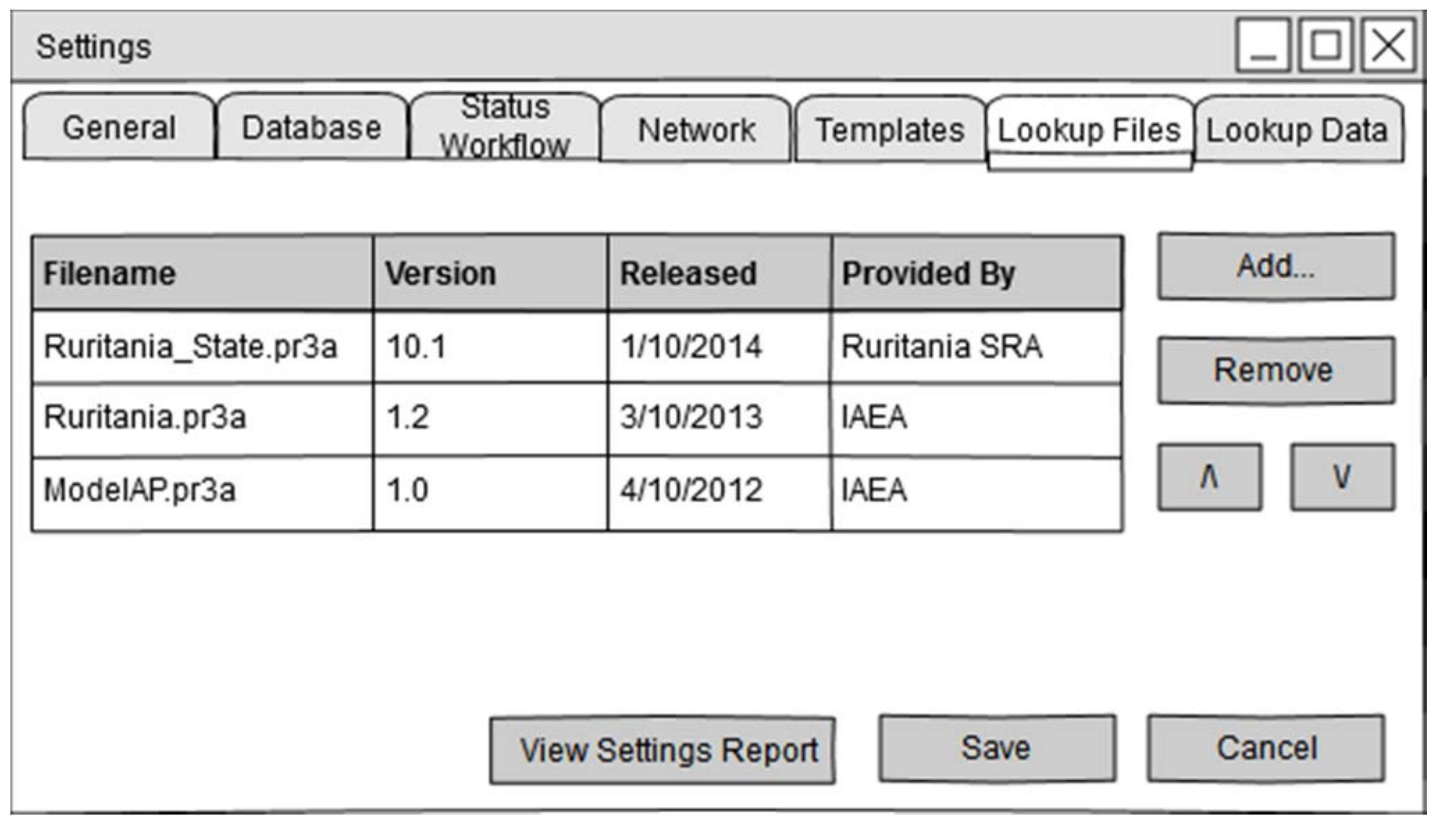

Fig. 24. The Lookup Files tab of the Settings window.

Since different lookup files may contain different values for the same ID, lookup files have different priorities. If there is a conflict, values from higher priority lookup files will override those from lower 
priority lookup files. It is unlikely this will affect most users, but the option should be there in case there is a conflict.

\subsubsection{Settings: Status Workflow Tab}

By default, all Statuses in the PR3 workflow (see Business Rules) should be available to the user. However, users should be able to hide selected Statuses as an option through Settings (Fig. 25) so that their copy of the PR3 reflects the workflow their State, SRA, or Contributor organization follows.

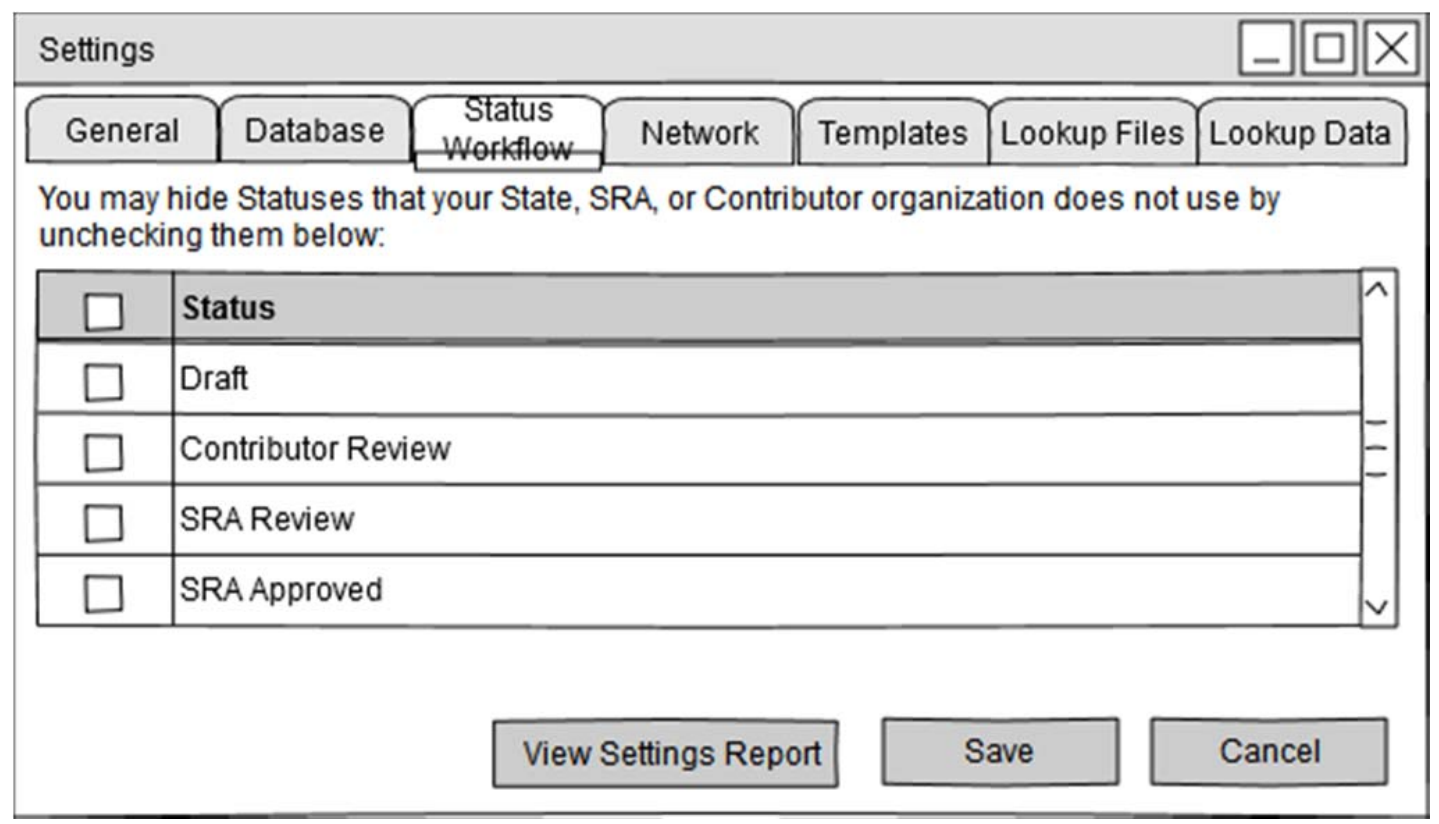

Fig. 25. The Status Workflow tab of the Settings window.

Unchecking a Status will remove it as an option from all Status drop-down lists (except when opening Entries and Submissions that are currently set to that Status). It will not affect the functionality of reports or data grids. Checking a Status will restore it as an option to all Status drop-down lists.

\subsubsection{Submission/Declaration Editor Window}

The Submission Editor window is used to edit Submissions and the Declarations it contains. The layout should clearly communicate the following:

- The separation of internal data used by the State from declarable data sent to the Agency

- The separation of "header" fields used for identification from "detail” fields that are filled in to meet AP requirements

- How to save, validate, and print the Submission at any time

- How to add, remove, and reorder Declarations

- How to add, remove, and renumber Entries 
The design should prevent the breadth and granularity of the data collected by the PR3 from overwhelming the user. Wherever possible, the Submission Editor should make use of UI elements such as expanders and tabs to highlight the most commonly used fields while making it clear how to find other fields as necessary (Fig. 26).

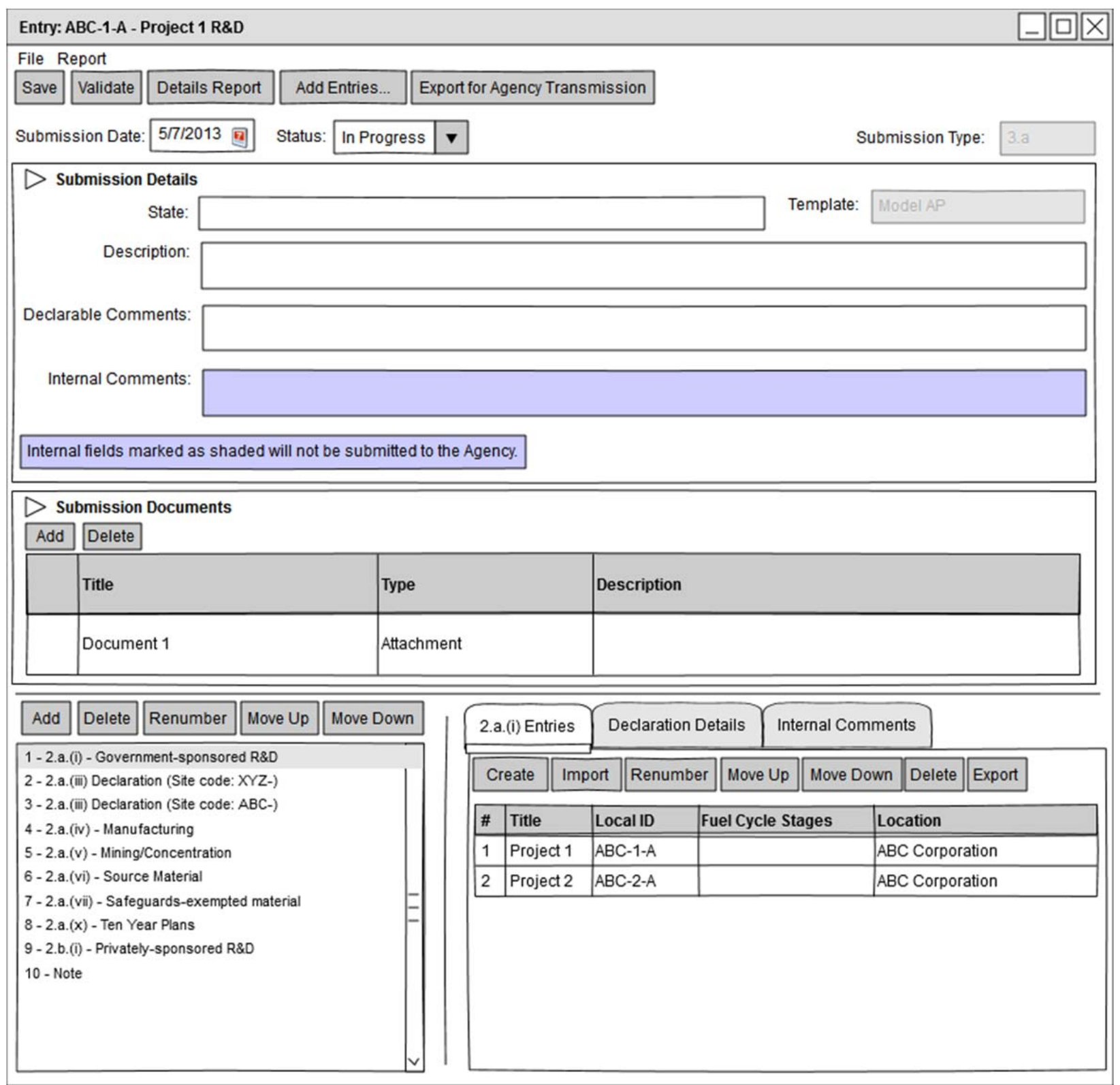

Fig. 26. The Submission Editor with a Declarations Entries tab selected.

Each Declaration in the Submission should contain one or more tabs for the Entries contained in that Declaration. Each of these tabs should correspond to one AP article. For example, Model AP 2.a.(i) Declarations will have only one Entries tab, while Model AP 2.a.(vi) Declarations may have up to three Entries tabs for the (a), (b), and (c) sub-articles.

Entries should be listed in a read-only grid that contains general identifying information (such as Title and Local ID), as well as a few short, meaningful article-specific fields. The user should be able to use a 
context menu or double-click a row to view or edit the selected Entry using the Entry Editor form (Sect. 2.4.5). The user should also be able to organize Entries within each Declaration from this screen, including reordering them, exporting them as independent Entries, or copying and moving them between Declarations.

The Submission Editor should rely on the Entry Editor as much as possible to keep its interface simple and prevent redundancy. It should only provide capability to edit an Entry in relation to its parent Declaration, such as setting its Entry number.

Each Declaration should also have a Declaration Details tab (Fig. 27). This should allow the user to set header information such as the reporting period and comments and add attached or referenced documents.

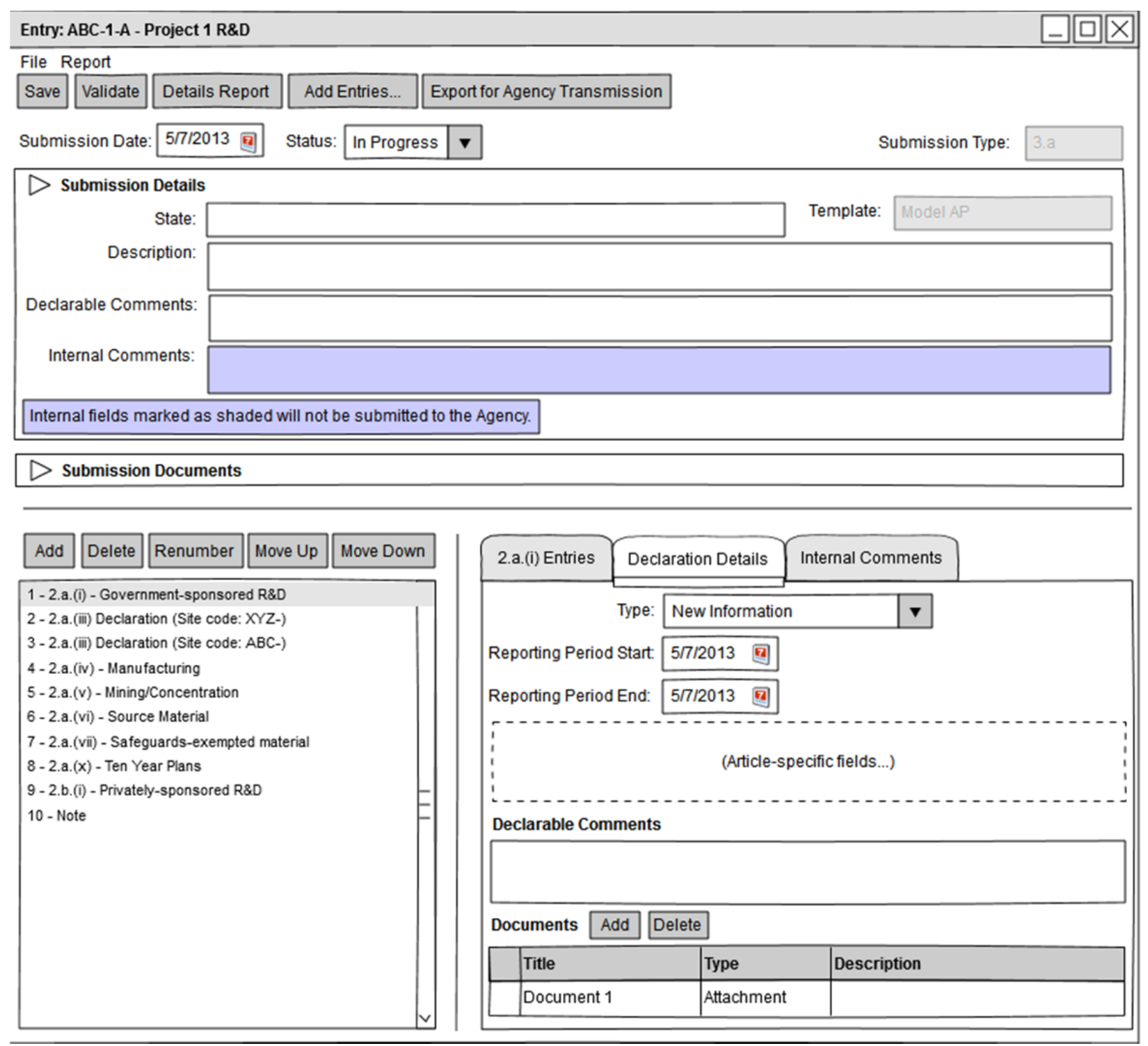

Fig. 27. The Submission Editor with a Declaration's Declaration Details tab selected.

This tab should be able to load additional controls from the data template plugin (Sect. 3.2) where necessary. For example, Model AP 2.a.(iii) and Model AP 2.c Declarations contain additional detail fields that are specific to those articles. In most cases, a Declaration will only have a few non-standard fields, so 
additional tabs are not warranted and the controls can be added directly to the Declaration Details tab. For example, in Windows Presentation Foundation, it should be sufficient to allow data templates to provide a class that extends the Panel class.

As in the Entry Editor, all internal Declaration data should be kept on a separate tab, so the division between internal and declarable data is clear (Fig. 28).

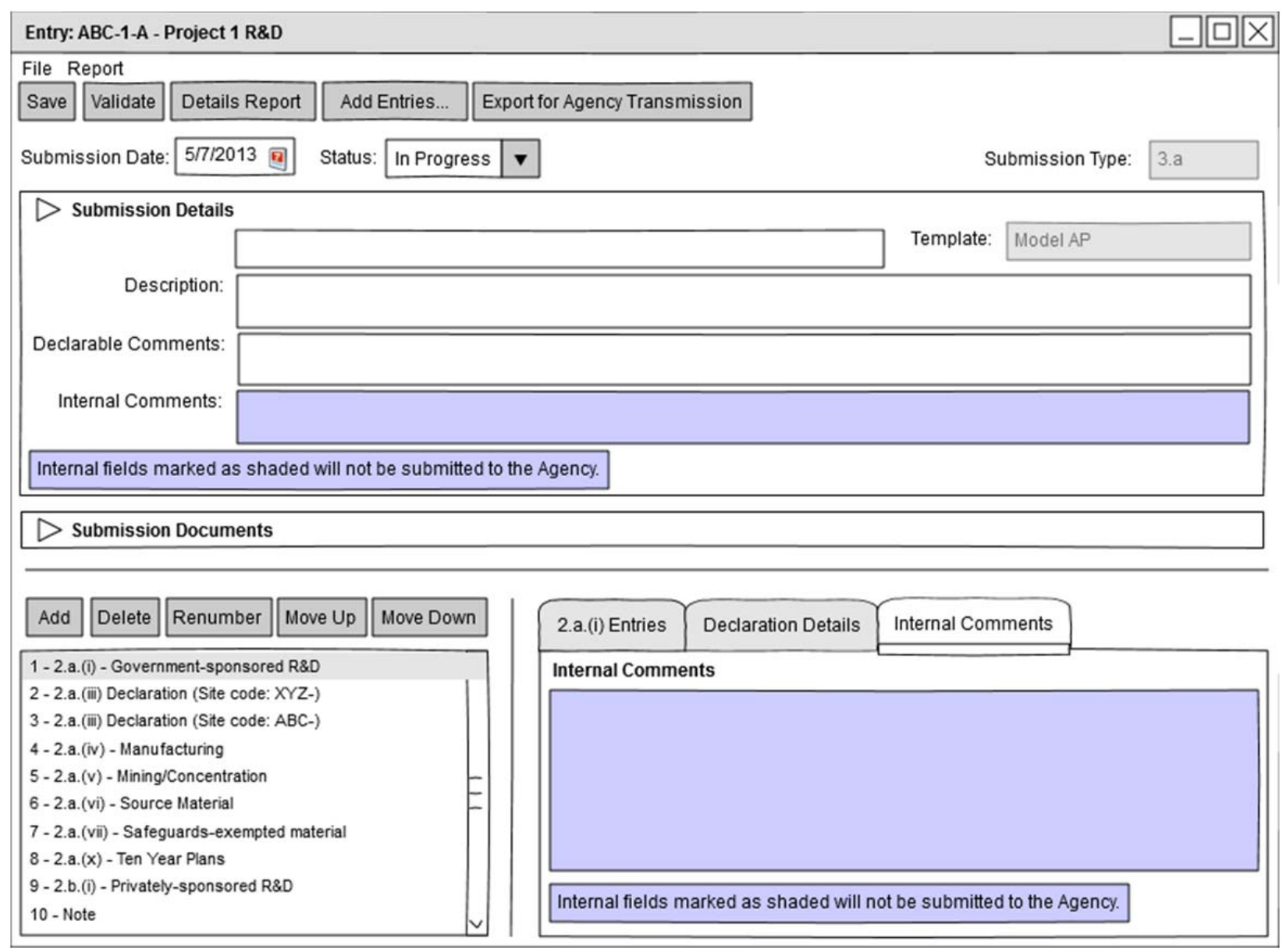

Fig. 28. The Submission Editor with a Declaration's Internal Comments tab selected.

\subsubsection{View Legacy Data Window}

Data imported into the PR3 from PR1 or PR2 Submission exports should initially be shown in read-only form in a layout similar to that of the PR2 (Fig. 29). 


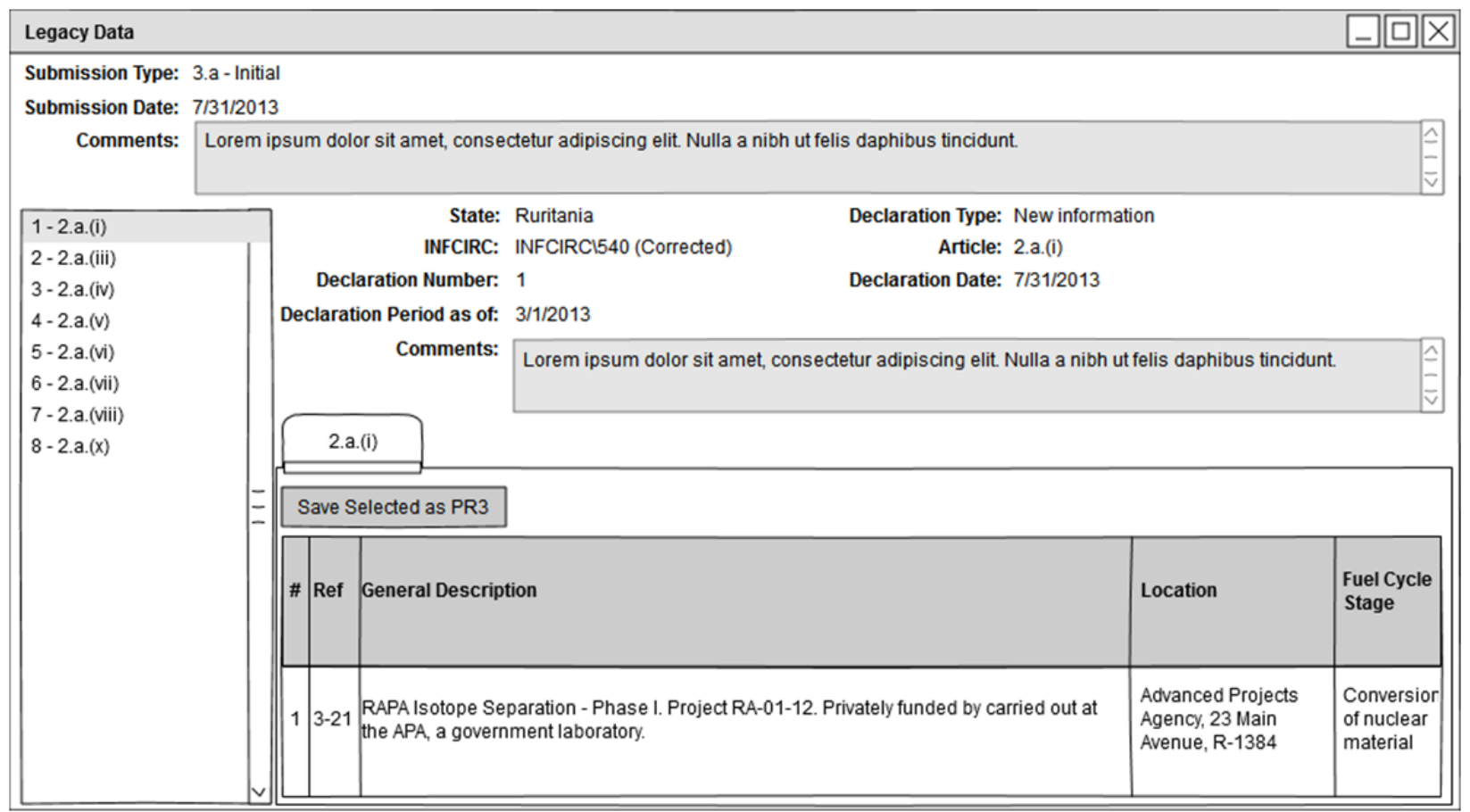

Fig. 29. The View Legacy Data window.

The user may view one Declaration from the Submission at a time. A list of Declarations should be shown in the left pane for navigation.

The functionality of this window is strongly tied to the capabilities of the data template. The Legacy Data feature relies on the data template to perform the imports. The more data template-specific functionality is built into the View Legacy Data window, the more functionality plugins will need to provide.

Because most PR1/PR2 Entries are less granular than their PR3 counterparts and may not fully validate properly, the user must explicitly choose to convert legacy data to PR3 format. This should be provided in the form of "Save as PR3 Entries," "Save selected as PR3 Entries," and "Save as PR3 Submission" commands. (This will typically be necessary only if the user migrates to the PR3 in the middle of declaration development, which is not expected to be a common use case.)

\subsection{REPORTS}

Reports in the PR3 serve two purposes: providing a summary of a particular type of data within the PR3 and providing a printable or exportable copy of data created in the PR3.

\subsubsection{Due Dates Report}

The Due Dates Report will display a list of all Submission due dates and reporting periods for the given period, given an Entry Into Force and/or Initial Submission As-Of Date (Fig. 30). 


\section{Due Dates Report}

Entry Into Force: 2012-08-28

As-Of Date:

2012-09-01

Report Dates:

2014-08-28 to 2016-08-28

\begin{tabular}{|c|c|c|c|c|c|}
\hline Submission & Description & Due Date & Reporting Period & Articles & Due Date Rule \\
\hline 3.d & $\begin{array}{l}\text { Quarterly } \\
\text { declaration (Annex } \\
\text { Il exports) }\end{array}$ & 2014-08-29 & 2014-04-01 to $2014-06-30$ & 2.a.(ix)(a), Note & $\begin{array}{l}\text { Within } 60 \text { days at the end of each } \\
\text { quarter }\end{array}$ \\
\hline 3.d & $\begin{array}{l}\text { Quarterly } \\
\text { declaration (Annex } \\
\text { Il exports) }\end{array}$ & 2014-11-29 & $2014-07-01$ to $2014-09-31$ & 2.a.(ix)(a), Note & $\begin{array}{l}\text { Within } 60 \text { days at the end of each } \\
\text { quarter }\end{array}$ \\
\hline 3.d & $\begin{array}{l}\text { Quarterly } \\
\text { declaration (Annex } \\
\text { Il exports) }\end{array}$ & 2015-03-01 & $2014-10-01$ to $2014-12-31$ & 2.a.(ix)(a), Note & $\begin{array}{l}\text { Within } 60 \text { days at the end of each } \\
\text { quarter }\end{array}$ \\
\hline 3.b & $\begin{array}{l}\text { Annual declaration } \\
\text { for initial }\end{array}$ & $2015-05-15$ & $2014-01-01$ to $2014-12-31$ & $\begin{array}{l}\text { 2.a.(i), (iii), (iv), (v), } \\
\text { (vi)(a), (vii), (x), } \\
\text { 2.b.(i), Note }\end{array}$ & By May 15 covering previous year \\
\hline
\end{tabular}

Fig. 30. An example of a Due Dates report.

Entry Into Force and As-Of Date should be populated with default values as defined in Settings but can be changed by the user. The user should also be able to sort on each of the columns.

The report is intended primarily for users at the SRA who have AP-specific knowledge but should also be useful to Contributors. Details such as reporting periods and Entry/Declaration articles can guide users on developing declaration data. Details such as the method of determining the due date can help users understand the requirements of the AP.

The plugin for each data template must provide this data for the PR3 based on the text of Article 3 of each AP agreement.

\subsubsection{Entry Details Report}

The Entry Details Report will display a complete listing of visible data contained in Entries selected for inclusion in the report. This report should be available in two forms or should offer the option of including or excluding internal data (Fig. 31). 


\section{Entry Details}

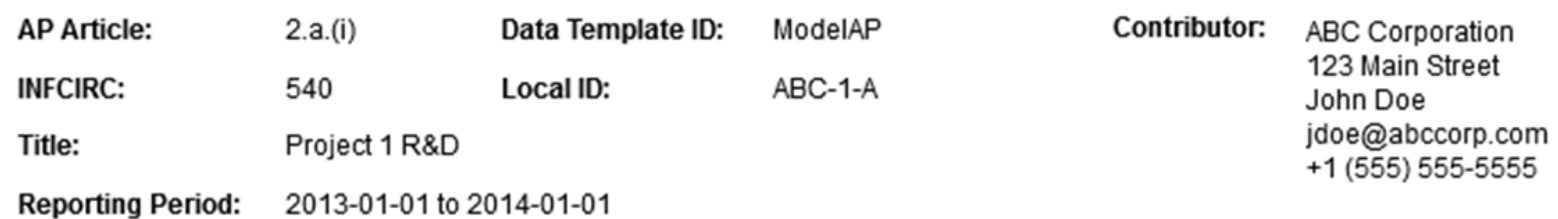

\begin{tabular}{|c|c|c|c|c|c|c|}
\hline \# & Entry Type & Ref. & Fuel Cycle Stage & Location & General Description & Comments \\
\hline 1 & $\begin{array}{l}\text { New } \\
\text { Information }\end{array}$ & $1-1$ & $\begin{array}{l}\text { Conversion of nuclear } \\
\text { material, Enrichment of } \\
\text { nuclear material }\end{array}$ & $\begin{array}{l}\text { ABC Corporation, } \\
123 \text { Main Street, } \\
24.68^{\circ} \mathrm{N} 12.34^{\circ} \mathrm{W}\end{array}$ & $\begin{array}{l}\text { Project } 1 \text { (ABC-1-A): Lorem } \\
\text { ipsum dolor sit amet, } \\
\text { consectetur adipiscing elit. } 1 \\
\text { Jan } 2013 \text { - } 1 \text { Jan } 2014 \\
\text { Objectives: Lorem ipsum dolor } \\
\text { sit amet consectetur adipiscing } \\
\text { elit. } \\
\text { Degree Met: } 90 \% \\
\text { Intended Application: Lorem } \\
\text { ipsum dolor sit amet } \\
\text { consectetur adipiscing elit. } \\
\text { Relationship of Activity to State: } \\
\text { Lorem ipsum dolor sit amet } \\
\text { consectetur adipiscing elit. }\end{array}$ & $\begin{array}{l}\text { Lorem ipsum } \\
\text { dolor sit amet } \\
\text { consectetur } \\
\text { adipiscing elit. }\end{array}$ \\
\hline
\end{tabular}

Fig. 31. An example of an Entry Details report.

Internal and identifying data meant for State use should be separated from declarable data that meets the requirements of the AP agreement. If possible, declarable data should match the format specified in "Guidelines and Format for the Preparation and Submission of the Declarations Pursuant to Article 2 \& 3 of the Model Protocol Additional to Safeguards Agreements (May 2004)” for consistency.

The plugin for each data template must define how article-specific data in the report is displayed.

\subsubsection{Settings Report}

The Settings Report will display a complete snapshot of all current settings for this installation of the PR3 (Fig. 32). This should include general settings, the current database, installed data templates and lookup files, and combined lookup data. This may be used to assist local information technology staff or the Agency in troubleshooting problems that users have with the PR3. 


\section{PR3 Settings Report}

Database

\begin{tabular}{|l|}
\hline Data Directory \\
\hline C:IUsersMyUsernamelDocumentsIDatabase \\
\hline
\end{tabular}

Data Templates

\begin{tabular}{|l|l|l|l|}
\hline Name & Filename & Version & Code \\
\hline Ruritania & IAEA.ProtocolReporter.Ruritania.dll & 3.0 & Ruritania \\
\hline Model AP & IAEA.ProtocoIReporter.ModelAP.dll & 3.1 & ModelAP \\
\hline
\end{tabular}

Lookup Files

\begin{tabular}{|l|l|l|l|}
\hline Filename & Version & Released & Provided By \\
\hline Ruritania_State.pr3a & 10.1 & $2014-01-10$ & Ruritania SRA \\
\hline Ruritania.pr3a & 1.2 & $2013-03-10$ & IAEA \\
\hline ModelAP.pr3a & 1.0 & $2012-04-10$ & IAEA \\
\hline
\end{tabular}

Fig. 32. An example of a Settings report.

\subsubsection{Submission/Declaration Details Report}

The Submission/Declaration Details Report will display a complete listing of data contained in a selected Submission (Fig. 33). This includes all visible data fields in the Submission, its Declarations, and its Entries. This report should be available in two forms or should offer the option of including or excluding internal data.

Internal and identifying data meant for State use should be separated from declarable data that meets the requirements of the AP agreement. If possible, declarable data should match the format specified in "Guidelines and Format for the Preparation and Submission of the Declarations Pursuant to Article $2 \& 3$ of the Model Protocol Additional to Safeguards Agreements (May 2004)” for consistency.

The plugin for each data template must define how article-specific data in the report is displayed. 
Submission Details

State: Ruritania

Declarable Comments

Lorem ipsum dolor sit amet consectetur adipiscing elit.

Internal Description

Lorem ipsum dolor sit amet consectetur adipiscing elit.

Internal Comments

Lorem ipsum dolor sit amet consectetur adipiscing elit.

\section{Declaration 1}

AP Article: 2.a.(i)

Declaration Type: New information

Reporting Period: As-of 2014-06-28

Declarable Comments

Lorem ipsum dolor sit amet consectetur adipiscing elit.

Internal Comments

Lorem ipsum dolor sit amet consectetur adipiscing elit.

\begin{tabular}{|c|c|c|c|c|c|c|}
\hline \# & Entry Type & Ref. & Fuel Cycle Stage & Location & General Description & Comments \\
\hline & $\begin{array}{l}\text { New } \\
\text { Information }\end{array}$ & 1-1 & $\begin{array}{l}\text { Conversion of nuclear } \\
\text { material, Enrichment of } \\
\text { nuclear material }\end{array}$ & $\begin{array}{l}\text { ABC Corporation, } \\
123 \text { Main Street, } \\
24.68^{\circ} \mathrm{N} 12.34^{\circ} \mathrm{W}\end{array}$ & $\begin{array}{l}\text { Project } 1 \text { (ABC-1-A): Lorem } \\
\text { ipsum dolor sit amet, } \\
\text { consectetur adipiscing elit. } 1 \\
\text { Jan } 2013 \text { - } 1 \text { Jan } 2014 \\
\text { Objectives: Lorem ipsum dolor } \\
\text { sit amet consectetur adipiscing } \\
\text { elit. } \\
\text { Degree Met: } 90 \% \\
\text { Intended Application: Lorem } \\
\text { ipsum dolor sit amet } \\
\text { consectetur adipiscing elit. } \\
\\
\text { Relationship of Activity to State: } \\
\text { Lorem ipsum dolor sit amet } \\
\text { consectetur adipiscing elit. }\end{array}$ & $\begin{array}{l}\text { Lorem ipsum } \\
\text { dolor sit amet } \\
\text { consectetur } \\
\text { adipiscing elit }\end{array}$ \\
\hline
\end{tabular}

Fig. 33. An example of a Submission Details report.

\section{COMPONENT DESIGN}

The purpose of this section is to define how each subsystem within the PR3 should operate and how they should interact with each other, the user, and the computer.

\subsection{OVERVIEW}

PR3 functions may be divided into three broad categories (Fig. 34):

- Data management, which assists the user in searching and organizing their collection of PR3 data; 
- Declaration data editors, which allow the user to create and edit Entries, Declarations, and Submissions; and

- Settings management, which allows the users to configure their installation by managing lookup values, data templates, and other general settings.

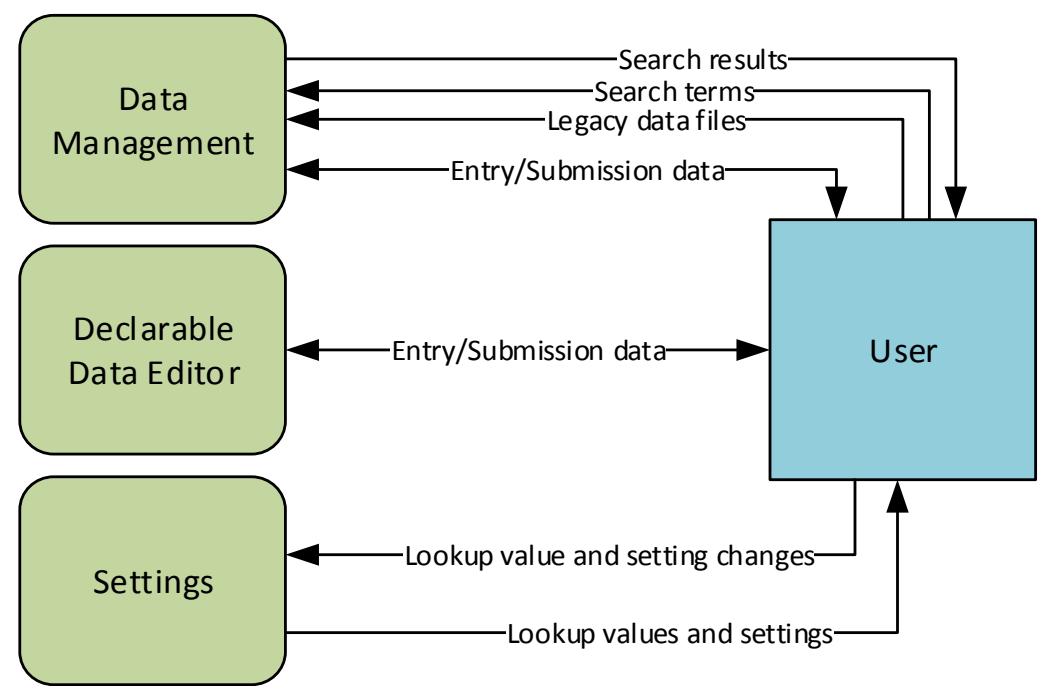

Fig. 34. The major categories of PR3 components. Each category provides different output to the user based on a different set of inputs. (Components must also provide information to each other, which will be illustrated in more detailed diagrams.)

\subsection{DATA TEMPLATES}

Because Member States' AP agreements can differ from the Model AP, the PR3 must be able to support data structures other than the Model AP. For flexibility, these templates will be implemented using a plugin architecture.

There are three goals for this design:

1. Distribute the PR3 with only the data template(s) needed by a user to avoid confusion and simplify usage.

2. Allow data templates to be created or changed without requiring an update to the PR3 itself.

3. Minimize the chance that changes to one data template may affect the behavior of another data template.

\subsubsection{Plugin Format and Development}

Each plugin will be implemented as a compiled library that the PR3 can load at run-time. In .NET, this means a DLL (dynamic link library) file. Plugins will have access to a common set of base classes for Entries, Declarations, and Submissions from which template-specific Entry, Declaration, and Submission classes must inherit. 
Plugins will also have access to composite data types, such as Foreign Collaboration, Location, etc., as defined in the SRS, Appendix C.1. They must also have access to the settings manager (Sect. 3.8) and lookup data manager (Sect. 3.7).

Fig. 35 is a class diagram and is an example of how the common library and a data template plugin should relate.

Aside from these requirements, a plugin may implement any additional classes as necessary. This allows a data template to include new functionality without having to update.

Plugins must implement a data template registration class which provides (at a minimum) the following:

- the plugin name and other identifying information

- the Entry, Declaration, and Submission types provided by the plugin

- the classes used to represent these Entry, Declaration, and Submission types

- the classes used to create and edit these Entry, Declaration, and Submission types

The PR3 will use this data template registration class to provide information about the template in the Settings window; options for creating new Entries, Declarations, and Submissions; and creating, loading, and exporting declaration data.

If the PR3 supports any additional "smart” functionality such as creating Update Entries and Submissions, suggesting default values, or providing step-by-step wizards, the plugin must provide the data templatespecific logic. The PR3 common library must provide interfaces that define how a data template plugin provides this logic. 


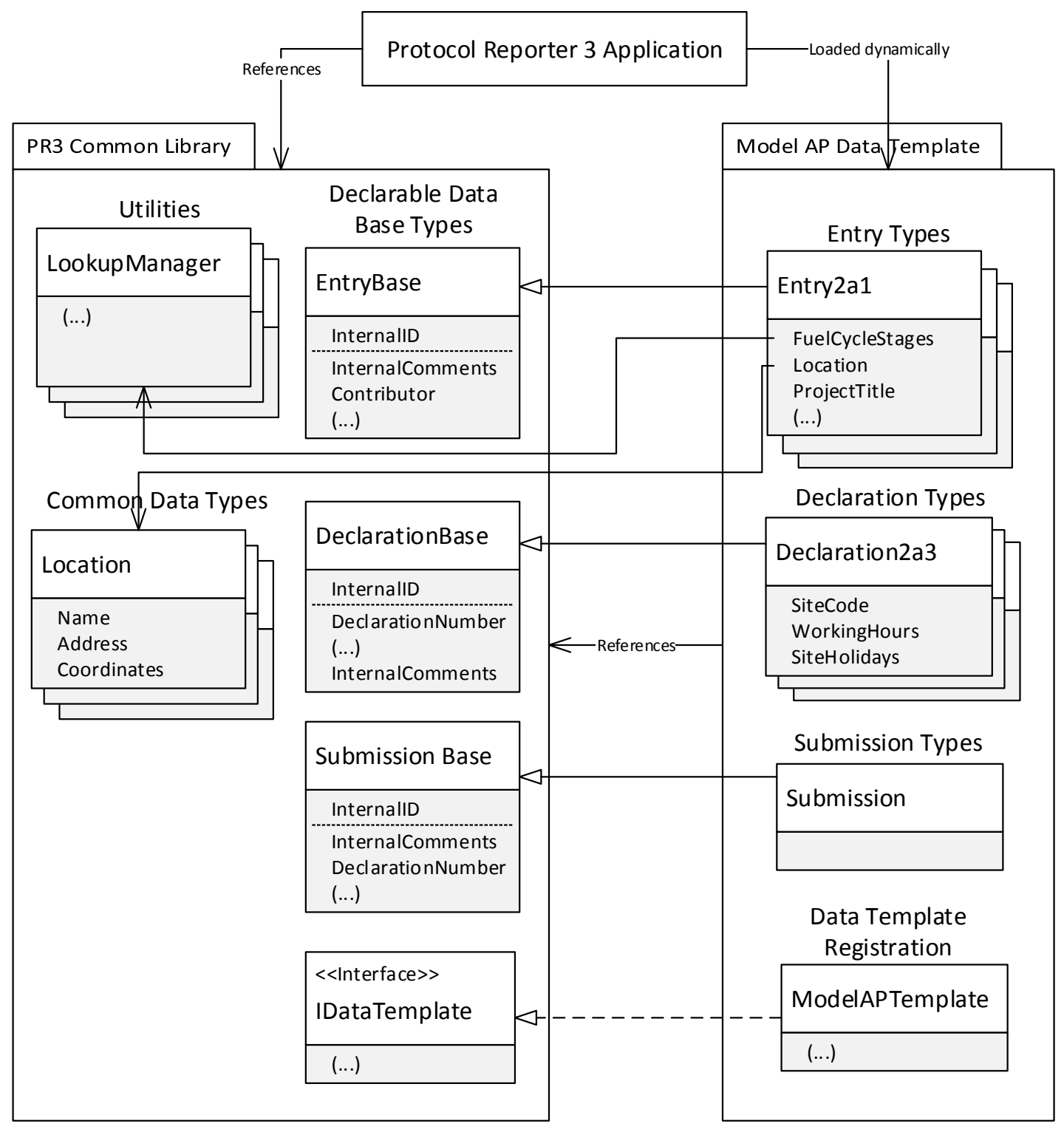

Fig. 35. An example of how assemblies/libraries and classes in the PR3 should relate to support data template plugins. This diagram is just an example, and the actual implementation may be different. A common library should provide base classes, interfaces, and classes that provide access to common PR3 functions. Data template plugins should implement as many of those base classes and interfaces as necessary.

\subsubsection{Managing Data Templates}

The PR3 will maintain a folder on the file system containing all installed plugins, and all DLLs in this folder will be automatically loaded if they include a data template registration class. Data template plugins will be installed for all users on the system; there will be no per-user data template installation. The PR3 should implement a plugin manager as a singleton, and other components that need to interact with template-specific code will do so through the plugin manager (Fig. 36). 


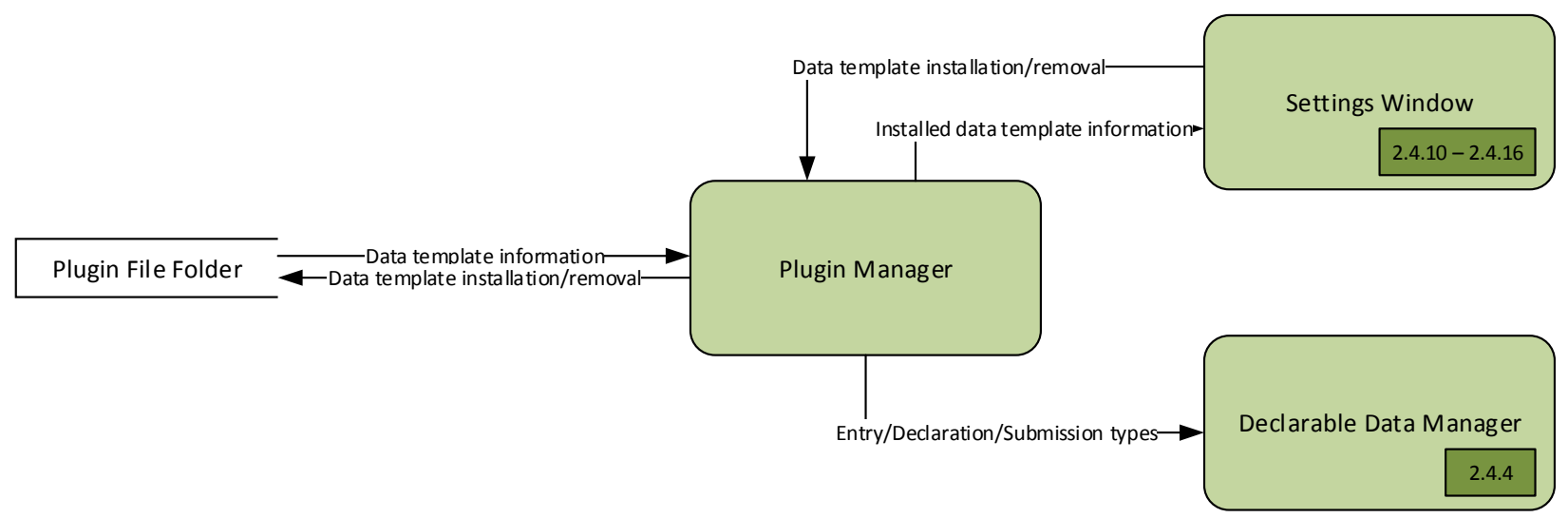

Fig. 36. The Plugin Manager component. This shows how the Plugin Manager stores and retrieves its data (to a file folder on the user's computer), and provides data to other components (Settings, Declaration Data Manager and Entry components).

The PR3 will contain the logic to handle abstract Entries, Declarations, and Submissions as well as edit basic fields common to all templates (such as identification and declaration reporting period fields). Plugins must provide user controls that can be inserted into Entry, Declaration, and Submission forms to edit template-specific fields.

For example, if a user creates a new Model AP 2.a.(i) Entry, the interaction between the PR3 and the Model AP data template would follow this pattern:

- The PR3 displays a Create Entry drop-down. The Model AP templates provide the list of available Entry types used to populate this drop-down.

- The Model AP template creates a new Entry2a1 object for storage. The Entry2a1 class inherits from the base Entry class defined in the PR3, providing the most basic Entry functions.

- The PR3 displays the Entry form with all common Entry fields. The Model AP template returns a new instance of the Entry2a1Editor control, which is used in the form.

- The Entry2a1Editor control contains controls defined in the PR3 to edit Foreign Collaboration and Location fields.

- When the user saves the Entry, a serializable object will be created that will be stored (either to file or to database). The PR3 generates the basic Entry structure, while the Model AP template generates the structure for the 2.a.(i)-specific details that will be inserted into it.

- When the user opens the Entry file, the PR3 will read the basic Entry structure and identify the template and Entry type. The Model AP will create a new Entry2a1 object. The PR3 will load all basic Entry fields, and the Model AP will load all 2.a.(i)-specific detail fields.

\subsection{ENTRY EDITING}

The PR3 will have a dedicated window for Entry editing tasks (Fig. 37). Since Entries in the PR3 are intended to collect structured, granular information, this replaces the grid-based approach to editing Entries in the PR2. (Entry data may still be summarized in grids, but this requires limiting or combining the fields shown to the user.) 
A new instance of the window will be opened for each Entry that is being edited, which allows the user to view and/or edit multiple Entries side-by-side. The Entry Editor window should have a consistent look and feel no matter the type or source of the Entry.

Because the Entry Editor must be flexible enough to handle all AP agreements, it must combine both common Entry fields with article-specific fields specified by the data template.

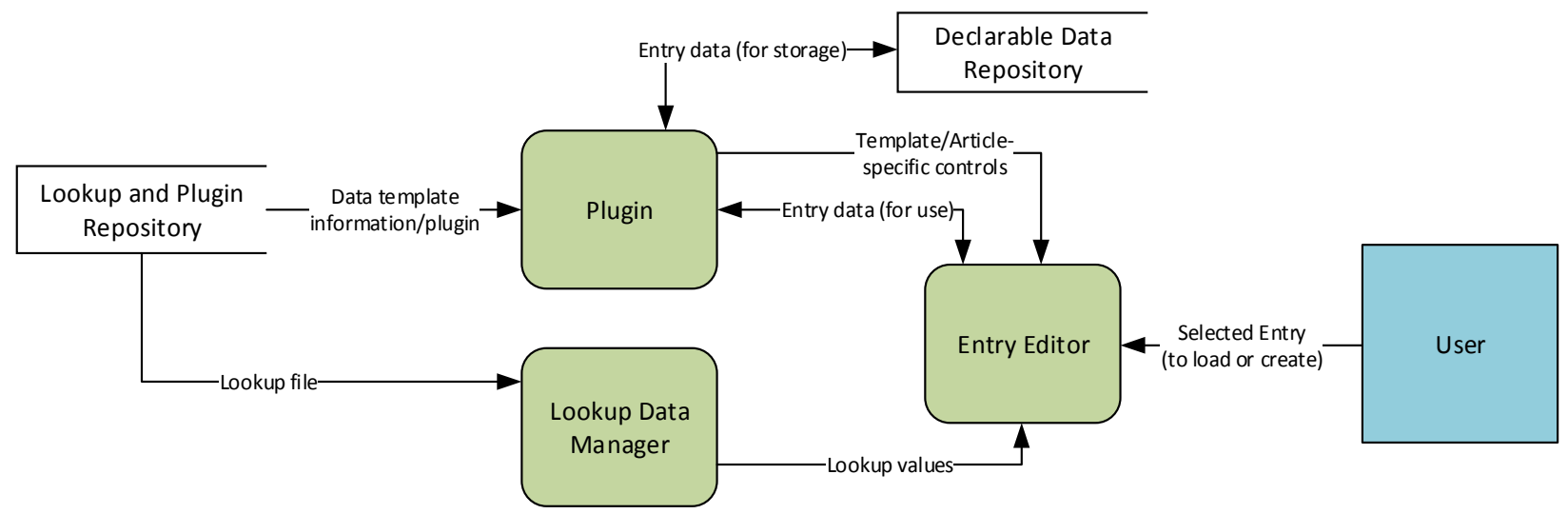

Fig. 37. The Entry Editor component. This shows how the Entry Editor component retrieves declaration data (from the database via a data template plugin) and data used to populate the UI (from the data template plugin and lookups).

When an Entry is created, it is associated with a selected data template and article, which cannot be changed. The Entry Editor must be able to adapt to the selected template and article for the loaded Entry.

\subsubsection{Use Cases}

There are multiple ways the Entry Editor window may be invoked, so it must support the following use cases:

- Creating or copying an independent Entry: An Entry is created, either blank or with certain prepopulated information. In this case, the Entry Editor window is provided a copy of the pre-populated Entry object. A new internal ID is generated for the Entry. The Entry may be saved by the user, in which case the Entry Editor must call the proper functions to perform the data access.

- Editing an independent Entry: An Entry is opened through the Data Manager. In this case, the Entry Editor window is provided nothing more than the Entry's internal ID and must call other functions to load the actual data.

- Editing an Entry within a Submission: A user edits an Entry from the Submission Editor window. In this case, the Entry Editor window is provided a copy of the Entry object as it exists in the Submission and is expected to return a copy of the modified Entry object back to the Submission Editor. Data access on the part of the Entry Editor is not necessary.

\subsubsection{Plugin Usage}

There must be a clean separation between Entry controls and data types included in the PR3 common library, and article-specific controls and data types included in the data template. 
The PR3 common library may provide base classes or interfaces from which data templates may inherit their article-specific controls or data types as necessary. Otherwise, common controls and data types in the PR3 should have no knowledge of what article-specific data may be included in an Entry.

To accomplish this separation, each plugin must provide the PR3 with methods that accomplish the following tasks. The Entry Editor window must determine the proper data template and then call the method from its plugin.

Examples of Entry Editor tasks for which a plugin must provide functionality are shown in Table 2.

Table 2. Examples of functions that a plugin must provide to the Entry Editor

\begin{tabular}{|c|c|c|c|}
\hline Task & Parameters & Returned value & Context \\
\hline Load an Entry & $\begin{array}{l}\text { Contents of the } \\
\text { stored Entry }\end{array}$ & $\begin{array}{l}\text { An Entry object that the } \\
\text { Entry Editor window } \\
\text { can load }\end{array}$ & $\begin{array}{l}\text { The PR3 must be able to read the } \\
\text { common elements of the stored Entry to } \\
\text { determine the data template plugin to use. }\end{array}$ \\
\hline $\begin{array}{l}\text { Retrieve controls necessary } \\
\text { to edit an Entry }\end{array}$ & $\begin{array}{l}\text { Entry Article } \\
\text { type }\end{array}$ & $\begin{array}{l}\text { A set of control types to } \\
\text { be used by the Entry } \\
\text { Editor window }\end{array}$ & $\begin{array}{l}\text { Once the data template and article are } \\
\text { determined, the Entry Editor can retrieve } \\
\text { a set of data template-specific controls } \\
\text { that it can display in a common location. } \\
\text { (One possible Windows Presentation } \\
\text { Foundation implementation might require } \\
\text { the plugin to return an array of user } \\
\text { controls that inherit from TabItem, which } \\
\text { the Entry Editor would append to the end } \\
\text { of its TabControl.) }\end{array}$ \\
\hline Validate an Entry & Entry object & $\begin{array}{l}\text { A set of validation } \\
\text { results }\end{array}$ & $\begin{array}{l}\text { The common library will define data } \\
\text { types for validation results. }\end{array}$ \\
\hline \multirow[t]{2}{*}{ Save an Entry } & Entry object & & $\begin{array}{l}\text { The data template must convert the Entry } \\
\text { object to a format that can be stored to } \\
\text { disk and loaded by the plugin. }\end{array}$ \\
\hline & & & $\begin{array}{l}\text { The PR3 common library should handle } \\
\text { converting common Entry fields and } \\
\text { saving the data to disk, but the plugin } \\
\text { must convert data template-specific fields. }\end{array}$ \\
\hline
\end{tabular}

\subsubsection{Editor Control Behaviors}

When creating user controls to be used in editing Entries, they must support all possible states and operations that the Entry Editor window supports.

- Read-only mode: It must be possible to set a user control to read-only mode. This will set all contained user controls to read-only mode and ensure no data can be changed. It should be possible to toggle this setting after the control has been loaded.

- Focus: It should be possible to set focus on a user control. (For example, this could be used to take the user to the field referenced by a validation message.) 


\subsection{SUBMISSION AND DECLARATION EDITING}

The PR3 will have a dedicated window for Submission and Declaration editing tasks. Because Declarations may not exist independently of Submissions, and because Submission-specific data is minimal, there is no reason to make these separate windows.

A new instance of the window will be opened for each Submission that is being edited, allowing the user to view and/or edit multiple Submissions side-by-side. The Submission Editor window should have a consistent look and feel for all Submission Types, except where necessary for data or workflow differences.

Because the Submission Editor must be flexible enough to handle all AP agreements, it must combine both common Submission and Declaration fields with article-specific fields specified by the data template. This requires the Submission Editor to interact with a data template plugin and the lookup data manager (Fig. 38). However, because Submissions and Declarations vary much less from article to article and template to template than Entries do, the impact is not as serious. Most Submissions and Declarations may contain the exact same fields, but they only differ in which columns are shown in the Entries grid.

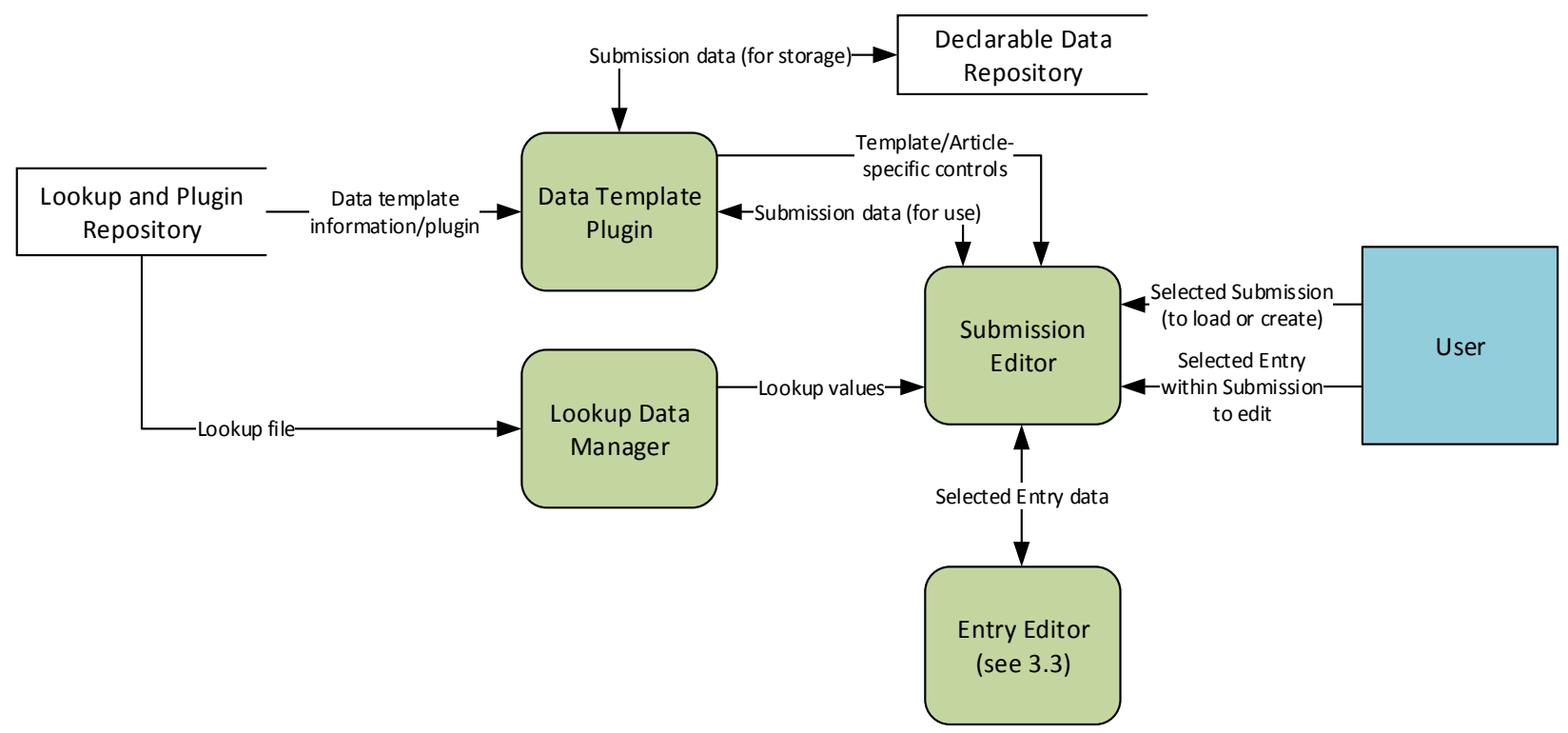

Fig. 38. The Submission Editor component. This shows how the Entry Editor component retrieves declaration data (from the database via a data template plugin) and data used to populate the UI (from the data template plugin and lookups). It also shows how editing Entries is passed off to the Entry Editor.

When a Submission is created, it is associated with a selected data template and article, which cannot be changed. The Submission Editor must be able to adapt to the selected template and article for the loaded Submission.

\subsubsection{Use Cases}

There are multiple ways the Submission Editor window may be invoked, so it must support the following use cases. (Note that because Submissions only exist as independent objects, the Submission Editor has less possible variation than the Entry Editor.) 
- Creating a Submission: A Submission is created either blank or with certain pre-populated information. In this case, the Submission Editor window is provided a copy of the pre-populated Submission object. The Submission may be saved by the user.

- Editing a Submission: A Submission is loaded from the Data Manager. In this case, the Submission Editor window is provided nothing more than the Submission's internal ID and must call other functions to load the actual data.

Additionally, the Submission Editor must handle multiple Submission Types. The PR3 must support data for each type as required in each State's AP agreement, but it should also minimize differences for easeof-use. The Submission Types that must be supported are:

- Initial Submissions (e.g., Model AP 3.a) represent the first set of Declarations submitted for a State. Typically, there is only one of these Submissions for each State. The PR3 can track the due date for an Initial Submission based on the State's Entry Into Force date. Declarations in an Initial Submission should not have "No Change" as an option for Declaration Type.

- Annual and Quarterly Updates (e.g., Model AP 3.b, 3.c, 3.d, and 3.e) represent Declarations that are submitted by a State on a regular basis. The PR3 can track the due date for an Annual or Quarterly Update Submissions based on the State's Entry Into Force date and the due date requirements defined in that State's AP agreement. There are two particular use cases of Annual and Quarterly Updates:

○ Submissions that may update previous Entries (e.g., Model AP 3.b) contain information about activities that may span multiple years. The PR3 should make it easy for users to use previously submitted data as a basis for these Submissions.

○ Submissions that only contain one-time Entries (e.g., Model AP 3.c, 3.d, and 3.e) contain information about single, one-time events such as imports and exports. An entirely new Submission must be created for each reporting period.

- Responses to Agency Requests (e.g., Model AP 3.f 3.g, and 2.c Response) represent Declarations that are submitted by a State only in response to an Agency request. The PR3 cannot predict due dates for future Agency requests; it must rely on the user to provide this information. Declarations in a Response to Agency Request Submission should not have "No Change" as an option for Declaration Type.

\subsubsection{Plugin Usage}

There must be a clean separation between Submission controls and data types included in the PR3 common library, and article-specific controls and data types included in the data template.

The PR3 common library may provide base classes or interface from which data templates may inherit their article-specific controls or data types as necessary. Otherwise, common controls and data types in the PR3 should have no knowledge of what article-specific data may be included in a Submission.

To accomplish this separation, each plugin must provide the PR3 with methods to perform the following tasks. The Submission Editor must determine the proper data template to use and then call the method from its plugin.

Examples of Submission Editor tasks for which a plugin must provide functionality are shown in Table 3. 
Table 3. Examples of plugin functions used by the Submission Editor

\begin{tabular}{|c|c|c|c|}
\hline Task & Parameters & Returned value & Context \\
\hline Load a Submission & $\begin{array}{l}\text { Contents of the } \\
\text { stored Submission }\end{array}$ & $\begin{array}{l}\text { A Submission object that } \\
\text { the Submission Editor can } \\
\text { load }\end{array}$ & $\begin{array}{l}\text { The PR3 must be able to read the common } \\
\text { elements of the stored Submission to } \\
\text { determine the data template plugin to use. }\end{array}$ \\
\hline $\begin{array}{l}\text { Retrieve controls as } \\
\text { necessary to edit a } \\
\text { Submission }\end{array}$ & $\begin{array}{l}\text { Submission Article } \\
\text { type }\end{array}$ & $\begin{array}{l}\text { An object that defines: } \\
\text { - A control type for } \\
\text { Article-specific } \\
\text { information in the } \\
\text { Submission header (if } \\
\text { necessary). } \\
\text { - A control type for each } \\
\text { valid Declaration type for } \\
\text { the Submission, for } \\
\text { Article-specific } \\
\text { information in each } \\
\text { Declaration header (if } \\
\text { necessary). }\end{array}$ & $\begin{array}{l}\text { Once the data template and article are } \\
\text { determined, the Submission Editor can } \\
\text { retrieve a set of data template-specific } \\
\text { controls that it can display in the Submission } \\
\text { and Declaration headers as necessary. } \\
\text { (One possible Windows Presentation } \\
\text { Foundation implementation might require } \\
\text { the plugin to return an array of user controls } \\
\text { which can be inserted into a StackPanel or } \\
\text { Grid.) }\end{array}$ \\
\hline $\begin{array}{l}\text { Retrieve columns as } \\
\text { necessary for editing } \\
\text { Entries within a } \\
\text { Declaration }\end{array}$ & Entry Article type & $\begin{array}{l}\text { A set of column names (or } \\
\text { column objects) that } \\
\text { defines Article-specific } \\
\text { information to show for } \\
\text { that Entry type. }\end{array}$ & $\begin{array}{l}\text { Each Declaration must show a list of Entries } \\
\text { (ideally as a data grid). To get more details, } \\
\text { the user will be able to open each Entry in } \\
\text { the Entry Editor. } \\
\text { Certain common identifying fields should be } \\
\text { shown in this grid by default (Local ID, } \\
\text { Title, etc.); however, data templates should } \\
\text { choose which Article-specific fields to show. }\end{array}$ \\
\hline Validate a Submission & Submission object & A set of validation results & $\begin{array}{l}\text { The common library will define data types } \\
\text { for validation results. }\end{array}$ \\
\hline Save a Submission & Submission object & XML content & $\begin{array}{l}\text { The data template must convert the } \\
\text { Submission object to a format that can be } \\
\text { stored to disk and loaded by the plugin. } \\
\text { The PR3 common library should handle } \\
\text { converting common Submission fields and } \\
\text { saving the data to disk, but the plugin must } \\
\text { convert data template-specific fields. }\end{array}$ \\
\hline
\end{tabular}

\subsection{SEARCH}

Ideally, users will maintain a collection of previous years' data in the PR3, as well as track and organize drafts for upcoming declarations. While grouping, sorting, and filtering functions (ideally, built into data grid views) can assist with this to a degree, a search function is critical to working with such a large amount of data.

The main advantage of search is it allows users to search across large free text fields, whereas most filtering functions are limited to short free-text fields or fields with a fixed set of choices. For this reason, the search feature should be tailored to full-text searching with the option to perform searches on one or more specific fields. This is important because it does not overlap with functionality already available in filtering, grouping, or sorting. To accomplish this, the search function must interact with data template plugins to retrieve and parse State- and article-specific data (Fig. 39). 


\subsubsection{Architecture}

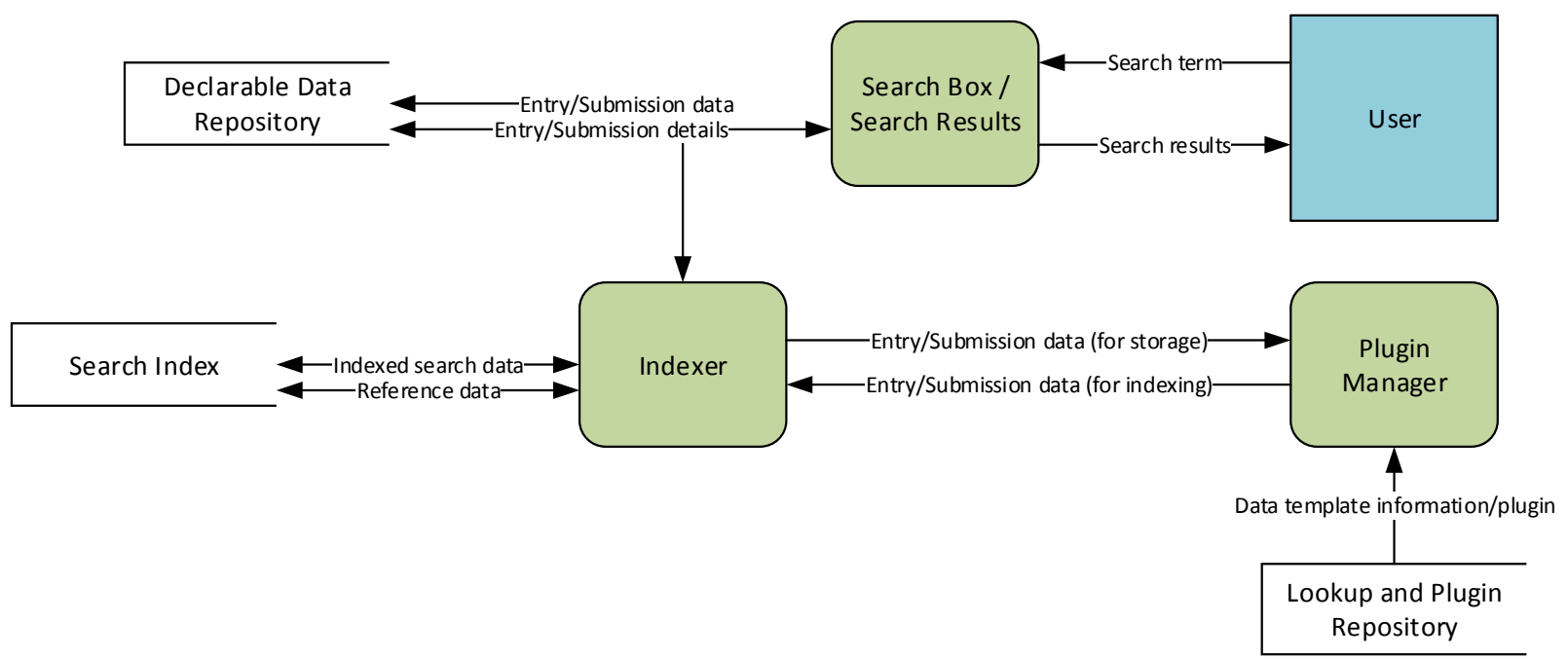

Fig. 39. The Search component. This shows how Search must retrieve declaration data to index (from the database, using a data template to determine how it should be indexed), save temporary index data to disk (if necessary), and return results to the user.

Because developers may wish to control how Entries and Submissions are indexed for full-text searching, the search index may be separate from the mechanism used to store PR3 data.

In this case, the PR3 will have its own indexer running in a separate thread. When an Entry or Submission is created, edited, or deleted, it will be re-indexed. The indexer will store searchable data linked to the Entry or Submission's internal ID. (The indexer may also be used for collecting other data not used in searches; for example, it should create an index of Internal IDs, Titles, and Local IDs that can be used when creating and validating References.)

This index should be saved to the user's local application data directory. If it is ever corrupted or deleted, it can easily be rebuilt from scratch as it should never be used to store or retrieve declaration data (only to perform searches). The user should also have the option to rebuild the index explicitly for troubleshooting purposes.

The indexer may also be used for collecting other data not used in searches; for example, it should create an index of Internal IDs, Titles, and Local IDs that can be used when creating and validating References.

When a search is performed, it is sent to the indexer, which returns a list of internal IDs for the Entries or Submissions that match the search. The window that shows search results can then use the internal IDs to retrieve additional information such as Title, Local ID, Article, Data Template, etc. (much like the Data Manager does).

\subsubsection{Third-party Library Requirements and Recommendations}

It is recommended that a third-party utility be used for full-text searching. This utility should run in-process and require no additional installations (to meet requirement SA-005 that states no external database should be required). The suggested utility is Lucene.NET, which is open-source, allows for indexing and searching on arbitrary fields (useful because the structure of Entry data will vary greatly), and runs in-process from a DLL rather than as a separate service. See Target Tools (APPENDIX B) for more information. 


\subsection{LEGACY DATA}

To support States in transitioning from PR1 and PR2 to PR3, the PR3 must support importing PR1/PR2 data. There are two challenges involved in implementing this feature: PR1/PR2 store and export data into different file formats, and PR3 data templates are designed to be more granular than previous versions. Since PR1, PR2, and PR3 formats vary between AP agreements, data template plugins will be responsible for implementing both the PR1/PR2 import and the PR3 conversion (Fig. 40).

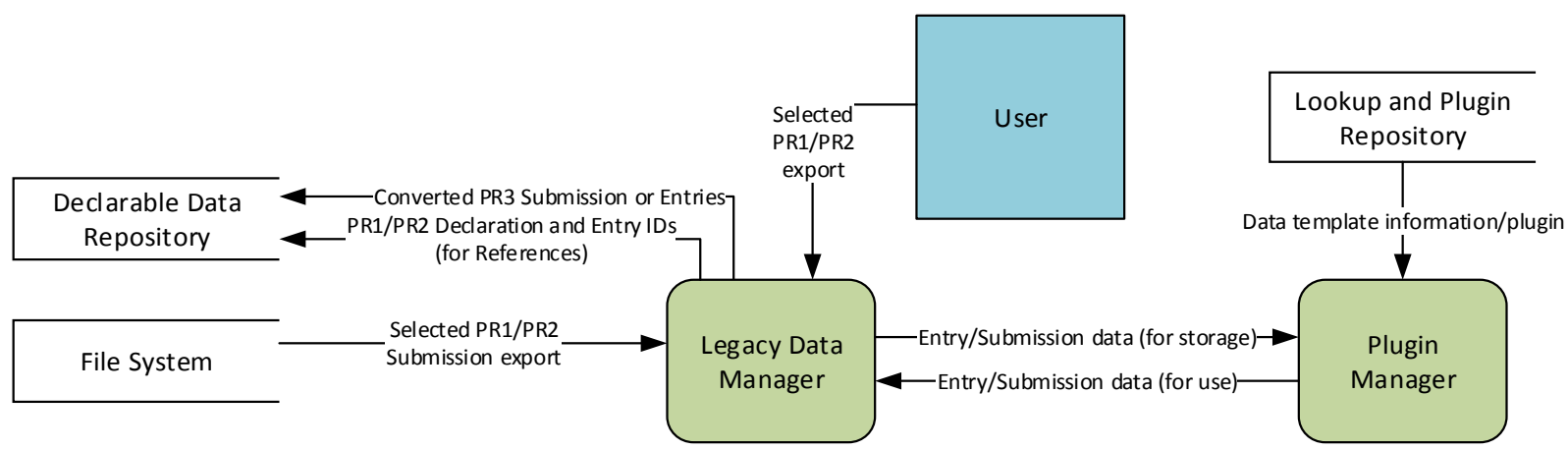

Fig. 40. The Legacy Data Component. This shows how Legacy Data should retrieve data from imported files and save it in PR3 format (using a data template in both cases).

The main goal of legacy data functionality is to provide States with a single, straightforward workflow for importing previous years' declaration data into the PR3. There are a few other potential use cases and workflows that seem to be edge cases.

\subsubsection{Supported File Formats}

The PR3 must support import of both PR1.0 comma-delimited format and PR2.0 Agency XML format. (This implies that all imports will happen on the Submission level.) While importing directly from the PR1 or PR2 database file may be technically possible, using export files should be easier for the user [especially for the PR2, where database access would require the user to install Structured Query Language (SQL) Server].

In both cases, data templates must support parsing and converting PR1 and PR2 files. This will particularly be necessary for PR2 Agency XML format where each type of Submission has a different schema.

To support this feature, the PR3 common library should provide a set of base classes for legacy Submissions, Declarations, and Entries. Data templates must extend these classes to support the legacy data import feature.

\subsubsection{Use of Imported Data}

There are two possible use cases for importing legacy data.

First, users may wish to import previously submitted declarations so that they have a copy for reference. This will enable PR3 to perform lookups or validation on entry and declaration references and may also be useful for a State's internal record keeping. In this case, users will not need to edit or validate the migrated data in PR3 format. 
Second, users may wish to import declaration data that was in progress but not submitted to continue work on it. In this case, users will need to edit and validate the data in PR3 format. This is considered an uncommon edge case because States should plan to transition to the PR3 between declaration cycles. Unlike the first use case, it requires user intervention to complete because some data in PR1 and PR2 format cannot be automatically parsed into the more granular PR3 format.

To satisfy the first use case while still allowing for the second use case, the PR3 should adopt a two-tier approach.

When data is first imported, it should be kept in a read-only PR1 or PR2 data structure rather than converted to the more granular PR3 format. Ideally, these data will be indexed as search terms and reference lookups although article-specific text will not be indexed as it would be for PR3 files. The PR3 must have an interface for viewing this data.

The user must have the option of importing this into an editable PR3 format. Each data template plugin will be responsible for supplying the logic to map PR1/PR2 data into PR3 format (if applicable for its associated State).

Once legacy data is converted into PR3 format, it is functionally the same as a Submission or Entry that was created in the PR3. It can be edited, transferred, or deleted.

Again, as the PR3 splits some PR1/PR2 fields into more structured or granular fields, the result may not validate under the PR3 format. The user will be warned that the data may need to be revised before submission because some data cannot be automatically parsed and converted. (See SRS Appendices E and $\mathrm{F}$ for more details.)

\subsection{LOOKUP DATA}

The term "Lookup Data" refers to any data that is loaded into the PR3 for use in the editing or validation of Entries, Declarations, and Submissions. Typical uses include lists of possible options (for drop-down lists, radio button groups, and checkbox lists), auto-completion (for combo box controls), or reusable entries (for complex data types such as Contributors or Locations that may be reused frequently).

There will generally be two types of lookup tables: Agency-provided and State-editable. State-editable lookup tables may be edited by the user as well as imported from lookup files provided by the Agency, SRA, and/or Contributor organizations. Agency-provided lookup tables are not editable within the PR3 and should be loaded from Agency lookup files. The PR3 should provide a single interface for retrieving all lookup data, no matter its source or purpose. (Fig. 41).

\subsubsection{Agency-Provided Lookup Tables}

Agency-provided lookup tables cannot be edited in the PR3 (see Sect. 2.4.12) because they represent information that the Agency requires. Some Agency-provided values may be provided by data templates rather than separate lookup files.

Examples of Agency-provided lookup data include 2.a.(vii) Exemptions, Fuel Cycle Stages, and Information Circulars (INFCIRCs). 2.a.(vii) Exemptions represent the only valid ways that materials may be exempted from safeguards, so States must not be able to submit a different value. Fuel Cycle Stages represent the only types of research and development (R\&D) activities that are declarable, and States must not submit activities that are not declarable. INFCIRCs represent Agency-assigned publication numbers. 


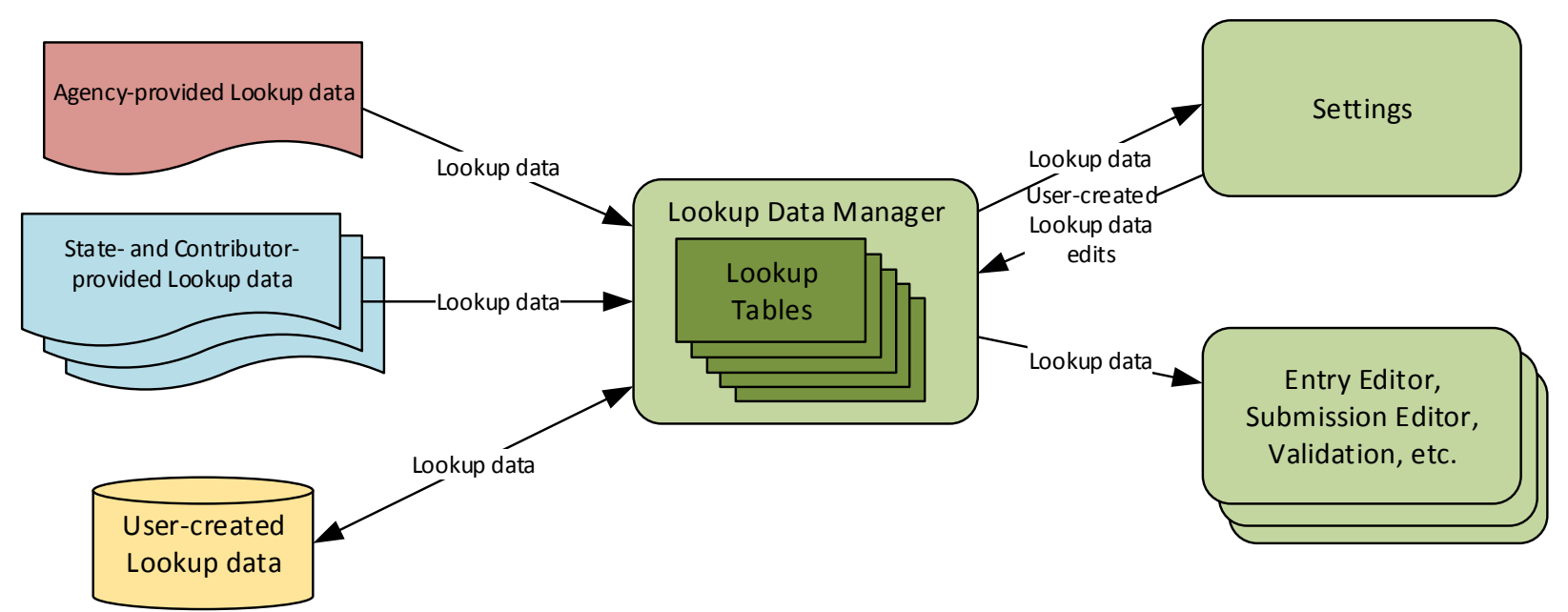

Fig. 41. The Lookup Data Manager component. This shows how the Manager combines data from different sources and makes it available to other components through a common interface.

Agency-provided lookup data may differ between States depending on the text of their AP agreements. For example, if a State's AP deviated from the Annex I list in the Model AP, then a different set of values for this lookup field would be needed. However, some data, such as the list of States or Fuel Cycle Stages, should be constant for all States and data templates.

Agency-provided lookup tables include the following:

- Annex I

- Annex II (including Nuclear Suppliers Group guidelines)

- AP Article

- Chemical Composition

- Declaration Type

- Entry Type

- Exemption

- Fuel Cycle Stages

- INFCIRC

- Intended Use Code

- Material Code

- Operation

- Operation Status

- Reference Type

- State

- Status

- Submission Type

- Unit of Area

- Unit of Quantity

When storing or submitting fields using Agency-provided lookup data, it is expected that other users and the Agency have a copy of this lookup list for validation. Only a unique identifying field needs to be stored or submitted. 


\subsubsection{State-Editable Lookup Data}

State-editable lookup data can be edited in the PR3 (see Sect. 2.4.12) because the State is typically the primary source of this information. The Agency may distribute lookup files for certain State-editable lists, but the State is able to add to or supersede these values.

Examples of Stated-editable lists include Annex I Voluntary Expansion and Facility/Location Outside Facilities (LOF) Code. Only the State can determine which manufacturing processes it is voluntarily declaring outside of Annex I, so only the State is responsible for this list. Although the Agency should know all valid Facility and LOF Codes for the State, the State may also update this list itself.

- $\quad$ Building ID

- Contributor

- Facility/LOF

- Location

- MBA Code

- Mine/Plant

- Site Code

When storing or submitting fields using State-editable lookup data, it is assumed that other users and the Agency do not have access to this data. All fields must be stored in the Entry or Submission.

\subsubsection{Storage and Transfer}

The PR3 must handle lookup data from two different sources.

First, all lookup data that has been created by the current user in the current installation of the PR3 must be saved to disk and reloaded at each startup. This data should be stored in the same database used to store Entry and Submission data.

When the PR3 is started, this lookup data will be loaded. If the file or database does not exist, it will be created with no lookup data included. Whenever changes are made, the file or database will be updated.

Second, exported lookup data must be stored as a file that can be passed between users and loaded into a different installation of the PR3. For ease of troubleshooting, this should be a readable text format such as XML.

Both Agency-provided and State-editable lookup data will use the same file format and procedure for lookup files, but the PR3 will only be able to export State-editable data. The Agency will use a separate utility for this process.

\subsubsection{Example Export File Format}

When lookup data (see Table 4) is exported, the user will be prompted first for a file path and filename and then for a version number and author name.

Export files should be in a human-readable format such as XML or JSON but will have the .pr3l (PR3 Lookup) file extension to identify its purpose.

If XML is used, it might look like the following: 


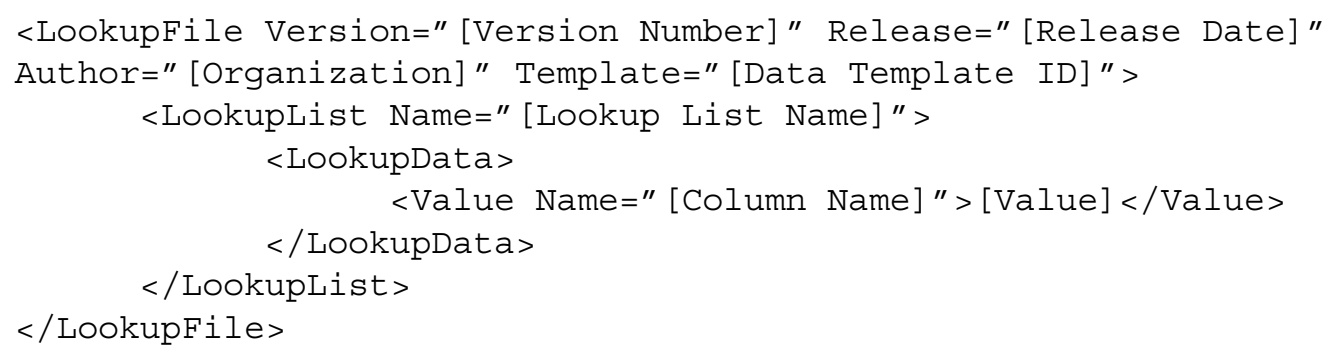

If JSON is used, it might look like the following:

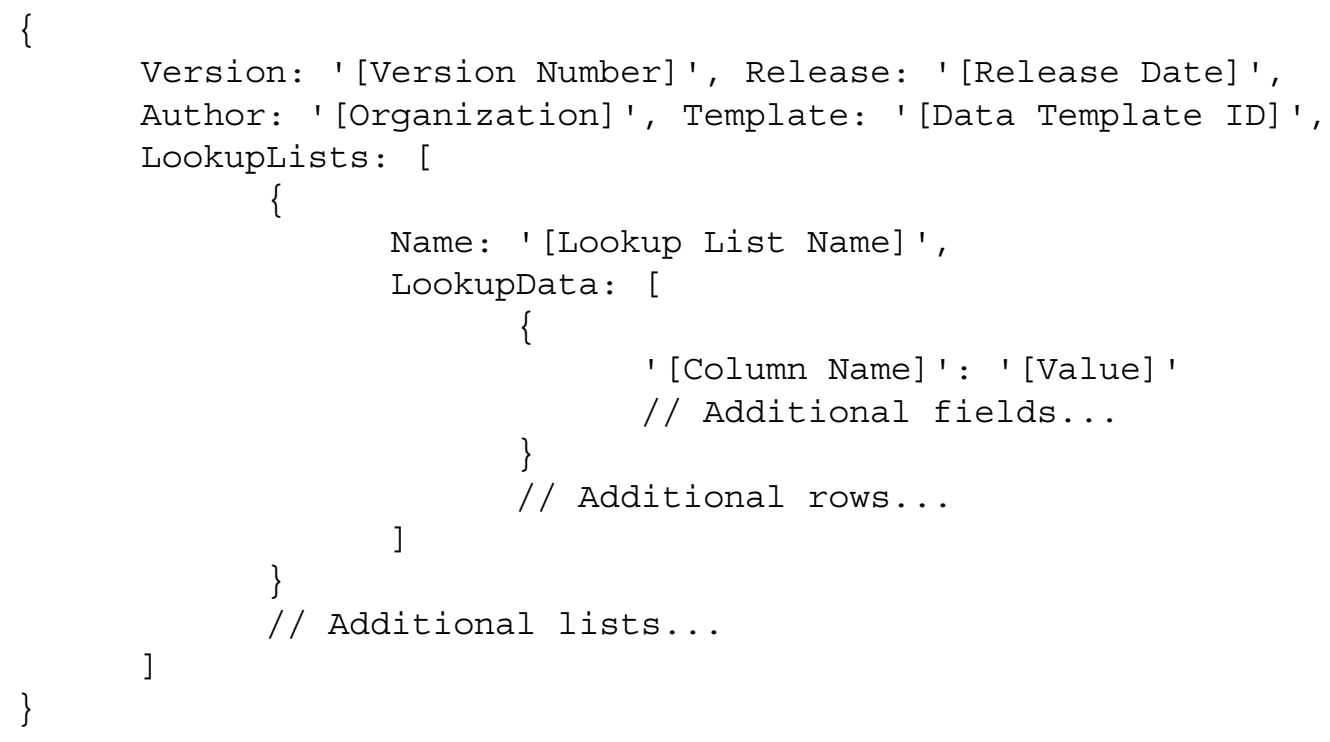


Table 4. An example of the structure of a lookup file

\begin{tabular}{|c|c|c|c|}
\hline Element or Attribute & Parent & Type / Constraints & Notes \\
\hline LookupFile element & None & $\begin{array}{l}\text { Element. One per } \\
\text { document. }\end{array}$ & The root note of the lookup file. \\
\hline Version attribute & LookupFile & String & $\begin{array}{l}\text { The version of the lookup file. Used to help } \\
\text { users identify which version they have installed. }\end{array}$ \\
\hline Release attribute & LookupFile & Date & $\begin{array}{l}\text { The date this lookup file was created. Used to } \\
\text { help users identify which version they have } \\
\text { installed. }\end{array}$ \\
\hline Author attribute & LookupFile & String & $\begin{array}{l}\text { The name of the organization that released this } \\
\text { lookup file. }\end{array}$ \\
\hline Template attribute & LookupFile & Optional & $\begin{array}{l}\text { The ID of the data template that data from this } \\
\text { lookup file is associated with. }\end{array}$ \\
\hline LookupList element & LookupFile & $\begin{array}{l}\text { Element. Zero, one, or } \\
\text { many. }\end{array}$ & $\begin{array}{l}\text { Defines a set of data that should be included in a } \\
\text { particular lookup list. }\end{array}$ \\
\hline Name element & LookupList & String & $\begin{array}{l}\text { The name of the lookup list that this element } \\
\text { should populate. }\end{array}$ \\
\hline LookupData element & LookupList & $\begin{array}{l}\text { Element. Zero, one, or } \\
\text { many. }\end{array}$ & $\begin{array}{l}\text { Defines a single row of lookup data to be } \\
\text { included in a lookup list. }\end{array}$ \\
\hline Value element & LookupData & $\begin{array}{l}\text { Element. Zero, one, or } \\
\text { many. }\end{array}$ & $\begin{array}{l}\text { Defines the value of a single column in a row of } \\
\text { lookup data. Contains the string value that } \\
\text { should populate that column. }\end{array}$ \\
\hline Name attribute & LookupData & String & The name of the column in a row of lookup data. \\
\hline
\end{tabular}

When loading lookup files in this format, the errors shown in Table 5 are possible.

Table 5. An example of how lookup file reading errors should be handled

\begin{tabular}{ll}
\hline \multicolumn{1}{c}{ Error } & \multicolumn{1}{c}{ Resolution } \\
\hline The LookupFile element does not exist. & $\begin{array}{l}\text { Fail to load the lookup file. Show a warning to the user. } \\
\text { Continue loading lookup files. }\end{array}$ \\
$\begin{array}{ll}\text { The Version, Release, or Author attributes do not exist } \\
\text { for LookupFile, or Release cannot be parsed as a date. } \\
\text { No Name is specified in a LookupList element, or the }\end{array}$ & $\begin{array}{l}\text { Load the lookup file, using a blank string for those } \\
\text { values. Show a warning to the user. }\end{array}$ \\
$\begin{array}{ll}\text { Name does not match a lookup list in the PR3. } \\
\text { No Name is specified in a Value element, or the Name lookup file, but ignore that LookupList } \\
\text { does not match a column in the specified lookup list. }\end{array}$ & $\begin{array}{l}\text { Load the rest of the LookupData element, but ignore that } \\
\text { Value element. Show a warning to the user. }\end{array}$ \\
\hline
\end{tabular}

\subsubsection{Usage}

PR3 will implement a lookup data manager as a singleton, which restricts instantiation to just one instance. The lookup data manager will be responsible for loading the user's lookup data, all loaded lookup files, and providing requested lookup data to all PR3 functions (Fig. 42). This ensures only one copy of lookup data is loaded at any given time, so there will be no discrepancies between PR3 functions. The lookup manager class should be included in the PR3 common library so that data template plugins have access to lookup data for use in forms and data objects. 


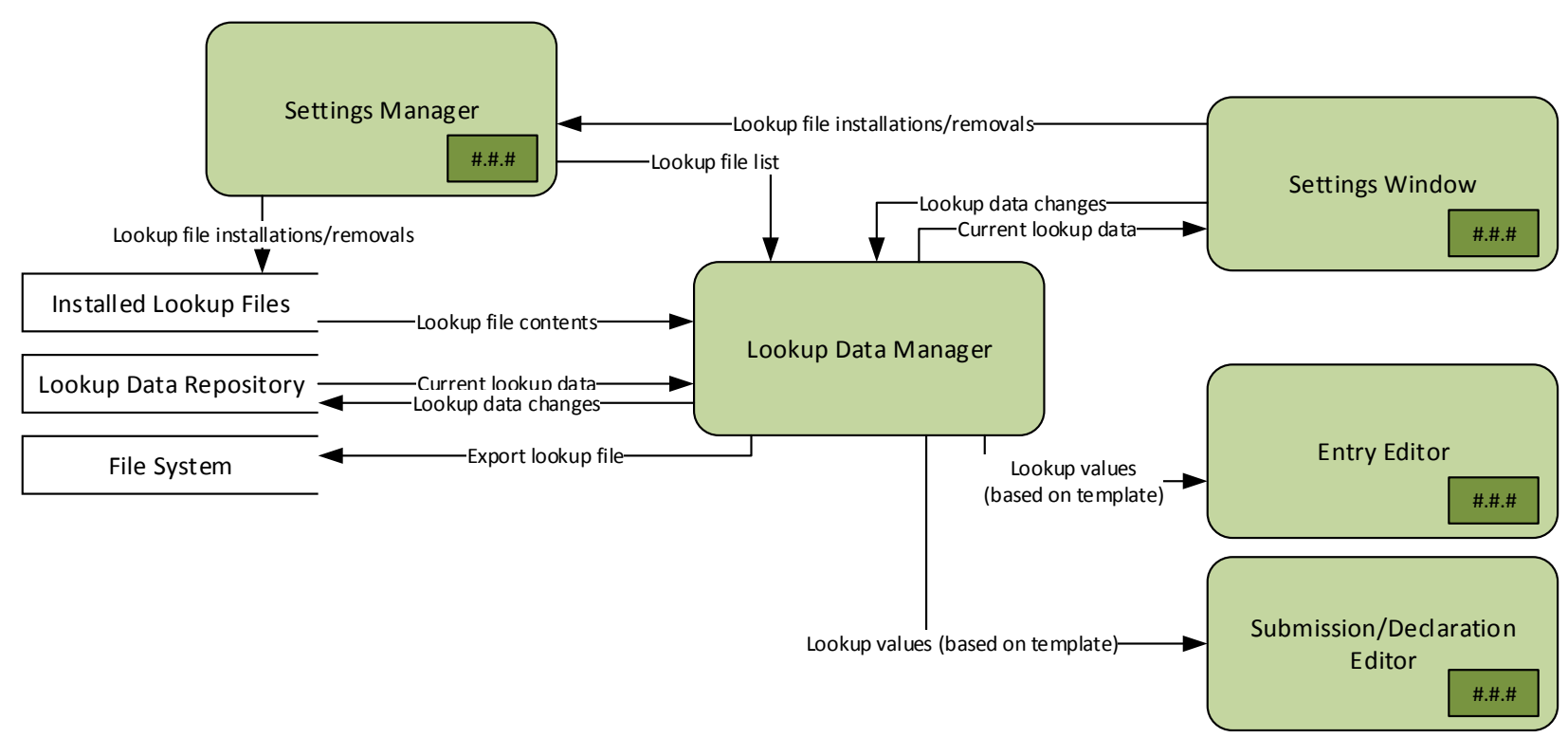

Fig. 42. The Lookup Data Manager component. This shows the way lookup data is retrieved from both imported lookup files and the current database and used by various components.

The lookup data manager will load the user's lookup data and all loaded lookup files on startup. Whenever the user changes lookup data, adds a new lookup file, or removes a lookup file, the lookup manager must update accordingly.

The lookup data manager must maintain the user's lookup data and each lookup file's data separately and remember the priority configured in the list of lookup files. The user's lookup data is always considered to have highest priority. When two lookup items in the same list share an ID, the lookup data manager will use the one with the highest priority. (This should be a rare case, but the lookup data manager must accommodate it.)

\subsubsection{Editing Lookup Data}

All State-editable lookup data may be edited through the Lookup Data tab on the Settings window (see Sect. 2.4.12). No Agency-provided lookup lists will be shown on this tab.

Whenever changes are made to the user's lookup data, all Entry, Declaration, and Submission forms must immediately reflect this. This will not update any loaded or exported lookup files; the user must explicitly re-export a new lookup file to do that.

\subsection{GENERAL SETTINGS}

\subsubsection{Managing Settings}

PR3 will have a single mechanism for storing all settings that do not fall under Lookup Data or Data Templates. This will serve as a general repository for any configuration options that do not warrant their own component or are identified during implementation (Fig. 43).

Configuration settings must be saved between PR3 sessions, so they must be saved to and loaded from a file. This file should be stored in the user's Windows roaming profile. Settings will be loaded from the file automatically upon startup. If a file does not exist at startup, one must be created with default values. If a file does exist but contains invalid values, the user will receive a warning, and the default value 
should be substituted for any invalid values. A new copy of the file will be written whenever changes are made to settings.

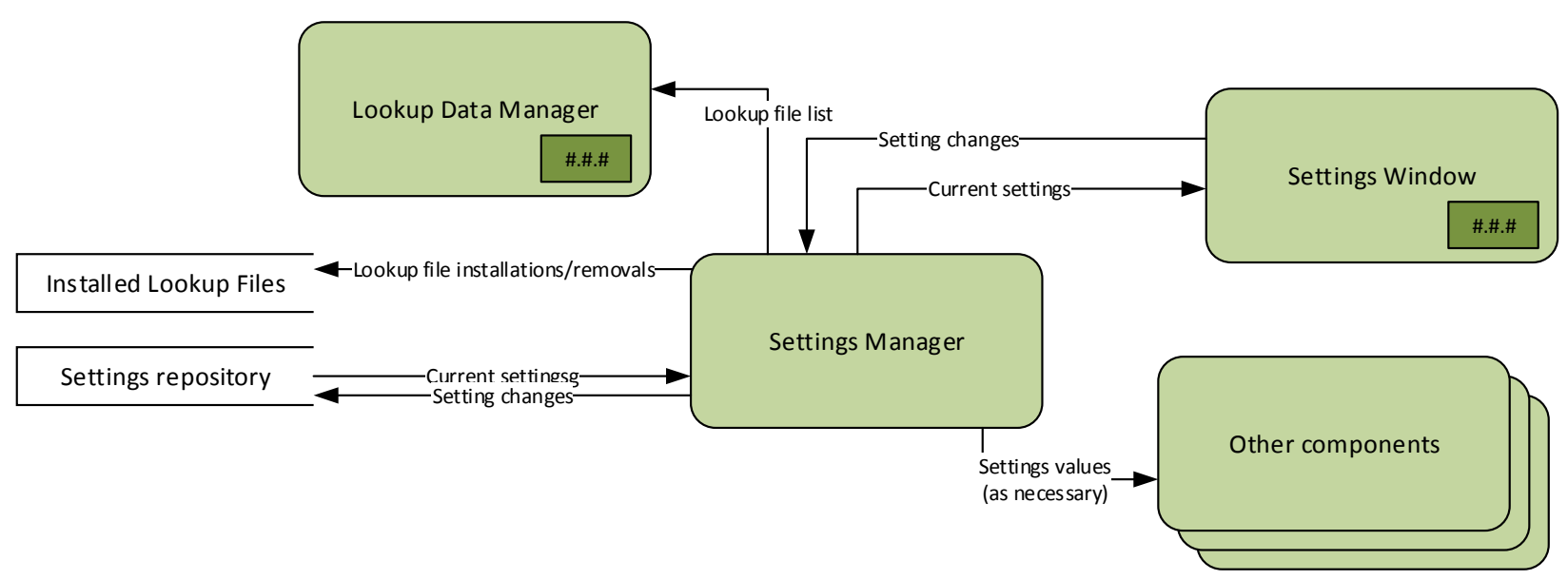

Fig. 43. The Settings Manager. This shows how the Settings Manager retrieves data from the database and provides data to other components.

PR3 will implement a Settings Manager as a singleton, and other components that need to retrieve settings values will do so through this class. This ensures that there is only one copy of the settings loaded at any given time and that it is always the most current version. The Settings Manager should be included in the PR3 common library so that plugins have access to settings data for use in forms and data objects.

\subsubsection{Settings Definitions}

For simplicity, settings should be saved and loaded using .NET serialization. For ease of troubleshooting, a text-based format such as XML or JSON should be used. Most settings will likely be no more complicated than a key-value pair, so there will be no need for complex data structures or custom serialization logic.

In Table 6, all settings are grouped by category. This should make it easier to document the purpose of and components affected by each Setting. This should loosely correspond to the tab in the Settings window.

Table 6. The list of Settings that must be included in the PR3

\begin{tabular}{lll}
\hline \multicolumn{1}{c}{ Setting Name } & Data Type & \multicolumn{1}{c}{ Notes } \\
\hline $\begin{array}{l}\text { General.Contributor Mode } \\
\text { General.User Name }\end{array}$ & $\begin{array}{l}\text { Boolean } \\
\text { String }\end{array}$ & $\begin{array}{l}\text { If true, SRA-specific features are hidden from the user. } \\
\text { The full name of the current user as it should appear in history } \\
\text { entries. If this is blank, the user will be prompted for this setting } \\
\text { on launch. } \\
\text { If a data template plugin does not provide a State name (because } \\
\text { it is not a State-specific template), this value should be used. It } \\
\text { may be selected from the State lookup list or entered as free } \\
\text { text. } \\
\text { If a dataldemplate plugin does not provide an Entry Into Force } \\
\text { date (because it was not known, or is not a State-specific } \\
\text { template), this value should be used. }\end{array}$ \\
$\begin{array}{l}\text { General.Default EIF (Entry Into } \\
\text { Force) Date }\end{array}$ & Date &
\end{tabular}


Table 6. The list of Settings that must be included in the PR3 (continued)

\begin{tabular}{|c|c|c|}
\hline Setting Name & Data Type & Notes \\
\hline General.Default AsOf Date & Date & $\begin{array}{l}\text { This should be used whenever the As-Of Date is pre-populated } \\
\text { and an existing As-Of Date is not available in other } \\
\text { Declarations. }\end{array}$ \\
\hline General.Default INFCIRC & String & $\begin{array}{l}\text { If a data template plugin does not provide an INFCIRC (because } \\
\text { it is not a state-specific template), this value should be used. }\end{array}$ \\
\hline $\begin{array}{l}\text { General.Default Starting } \\
\text { Declaration Number }\end{array}$ & Integer & $\begin{array}{l}\text { If the starting number cannot be determined from a previous } \\
\text { Submission, this number should be used when a Submission is } \\
\text { created. }\end{array}$ \\
\hline Network.Use Proxy ${ }^{*}$ & Boolean & If true, PR3 should use a proxy server for network access. \\
\hline Network.Proxy Address ${ }^{*}$ & String & The address of the proxy server. \\
\hline Network.Proxy Port ${ }^{*}$ & String & The port number of the proxy server. \\
\hline Network.Proxy SSL ${ }^{*}$ & Boolean & If true, the proxy server uses SSL encryption. \\
\hline Network.Proxy Auth ${ }^{*}$ & Boolean & If true, the proxy server uses authentication. \\
\hline Network.Proxy Auth Type* & Enum & $\begin{array}{l}\text { The type of authentication used by the proxy server. Valid } \\
\text { values are Basic, Digest, NTLM, and Windows. }\end{array}$ \\
\hline Network.Proxy Credentials Type ${ }^{*}$ & Enum & $\begin{array}{l}\text { The way credentials are passed to the proxy server. Valid values } \\
\text { are Stored, Prompt, or UseWindowsCredentials. }\end{array}$ \\
\hline Network.ProxyUsername ${ }^{*}$ & String & $\begin{array}{l}\text { The username for proxy server authentication, if Stored is } \\
\text { selected for Network.ProxyCredentialsType. }\end{array}$ \\
\hline Network.ProxyPassword* & String & $\begin{array}{l}\text { The password for proxy server authentication, if Stored is } \\
\text { selected for Network.ProxyCredentialsType. }\end{array}$ \\
\hline DataTemplate.Default & String & $\begin{array}{l}\text { The ID of the default data template. If not specified, this will be } \\
\text { the first one that is loaded. }\end{array}$ \\
\hline LookupFile.Priority & String[] & $\begin{array}{l}\text { A list of lookup filenames, in order of priority. Any filenames of } \\
\text { loaded lookup files not in the list will be added to the end of the } \\
\text { list in the order they are found. }\end{array}$ \\
\hline Database.Location & String & $\begin{array}{l}\text { The location of the database file. (If empty, a database should be } \\
\text { created on startup.) }\end{array}$ \\
\hline Statuses.Active Statuses & Boolean[] & $\begin{array}{l}\text { A flag for each PR3 status that determines whether it will be } \\
\text { shown as an option. Intended to allow users to customize their } \\
\text { interface to fit their State's workflow. }\end{array}$ \\
\hline
\end{tabular}

* Network settings will only be implemented if PR3 has any capabilities that require network connectivity, such as electronic transmission.

\subsection{LOGGING AND ERROR HANDLING}

PR3 must have a complete logging function to capture exceptions as well as any information that may be helpful in troubleshooting errors or other unexpected behavior. Ideally, a standard logging tool such as nlog or log4net should be used. Log files should be stored in text format in the user's local profile directory.

Unexpected exceptions must be caught (to prevent them from crashing PR3) and logged for troubleshooting purposes. If possible, a friendly explanation of the error will be shown to the user. For example, a .NET FileNotFoundException that occurs when loading the user's lookup data should trigger a message explaining what the purpose of the missing file is, where it might be found, and what might have caused it. It should not be a generic "file not found" error. 
If a friendly explanation is not available for an exception, the name and message for the exception should be shown to give the user some context. Unexpected exceptions must never be hidden from the user (as they may affect behavior of the application), and the user should never be shown a completely generic error message (e.g., "an error has occurred”).

\subsection{INTERNAL DATA STORAGE}

An in-process database should be used for persistence of data items managed by PR3. This database should not require any additional installation or licensing from the end user of PR3. See Target Tools (APPENDIX B) for recommended libraries that have been identified as meeting these requirements.

\subsection{PROGRAM INSTALLATION}

PR3 must come packaged as a Windows Installer (an .msi or .exe file) that will allow the user to choose options such as

- Installation location for PR3,

- Default mode of operation (i.e. Contributor or SRA mode), and

- Default data template (if multiple are included in the installation package).

PR3 must allow packaging of state-specific installation files that include the data templates and lookup files for specific states.

Source code can be made available separately from the distribution package.

\subsection{ITEM HISTORY}

To aid in collaboration and revision, the PR3 should keep a log of changes to Entries and Submissions. This log should be stored in the Entry or Submission object and should be included when the item is imported or exported.

At a minimum, Entries and Submissions should log

- the date and time of the change,

- $\quad$ the user who made the change (based on the User Name setting-see Sect. 3.8.2), and

- the nature of the action (creating the Entry/Submission, saving changes, status changes, etc.).

The Submission and Entry Editors should provide access to this log through a tab or a menu and toolbar command. This feature should be built into the data access components so that editors and data templates only have to provide minimal support for logging.

There are practical limitations to this feature:

- The granularity of the history may be adjusted depending on implementation. More detailed logging generates more data and would require support to be built into data template plugins, which may be impractical.

- Because there is no central repository of data within a State, an item may be copied and modified separately by multiple users. In this case, the complete history of the item may be split across its various copies. A user could consolidate data from multiple copies and resolve differences among them, but this would not consolidate the history. 
- To avoid complexity and confusion, history is only a record of changes. It need not allow a user to revert an Entry or Submission to a previous version.

\subsection{DATA EXPORT AND TRANSFER}

Since the design of the PR3 intends all data for an installation to be stored in a local database (Sect. 3.10), the user must be provided with tools for exporting data to a separate format for transfer.

There are two cases that must be handled: exporting data for transfer to other users' PR3 installations, and exporting data for transmission to the Agency. Depending on the database used for storage (APPENDIX B), there may be a third case: exporting a complete copy of the database for backup purposes.)

Both cases should be available to all users by default. However, since export for Agency transmission will likely be accessed through the Submission Editor (Sect. 2.4.15), it will not be accessible while a user is operating in Contributor Mode.

\subsubsection{File Format}

Export for both, other users and the Agency, should use a human-readable format such as JSON or XML. Ideally, both exports should use the same type of file although the structures of the files may differ. To support multiple languages, UTF-8 encoding should be used for all file exports.

To avoid some of the problems that affected PR2 import and export, PR3 should use a strict method for generating files [such as XML serialization based on an XML Schema Definition (XSD)] but should use a more lenient method for reading files [such as using the XML Document Object Model (DOM) or .NET's Language-Integrated Query (LINQ) to XML].

\subsubsection{Data Export for Users}

When data is exported for use by other PR3 users, it will be stored in a "Transfer Package" which allows one or more Entries or Submissions to be packaged into a single file. Typically, the SDS refers to this as "creating a transfer package" rather than "exporting" to avoid confusion with exports created specifically for Agency transfer.

When creating a transfer package, every piece of data in the Entry or Submission from the database should be stored in the file. This includes all internal data as well as the contents of any file attachments. Ideally, a user who imports an Entry or Submission from a transfer package should have an exact copy of what the user who exported it had at the time of export.

A user should not be prevented from creating a transfer package even if an Entry or Submission has validation warnings or errors. This allows a user to send data to another user while it is still in the process of being drafted.

A Transfer Package may be implemented as a single JSON or XML structure, or it could be implemented as a collection of JSON or XML files stored in a compressed archive such as a *.zip file. In either case, the JSON or XML component should conform to the requirements described in Sect. 3.13.1.

\subsubsection{Data Export for Agency Transfer}

When a Submission is exported for transmission to the Agency, it will be stored in a single JSON or XML file. Only declarable data (including the contents of any declarable file attachments) will be 
exported. Internal data will not be exported; the Agency transfer file format should not include structure for these fields.

Ideally, PR3 should be able to import a Submission exported for Agency transfer. However, because internal data is not included, it will not be a complete copy of all data items that contributed to the original Submission.

When exporting for Agency transfer, the user must export a single Submission in its entirety. Multiple Submissions must be exported as separate files, and Entries may not be exported independently of a Submission. If the Submission has validation errors, PR3 should not allow export for Agency transfer; however, validation warnings and informational messages are permitted.

\section{BUSINESS RULES}

\subsection{DECLARABLE STATUS WORKFLOW}

The purpose of this section is to define how Entry and Submission statuses may change in PR3. It does not define or prescribe a specific workflow for users. Instead, it provides a guide for developers to balance flexibility and ease-of-use in implementing these data fields so that users are free to use their own workflows.

\subsubsection{Overview}

Declaration data in the PR3 will have a Status field that allows users to track the Entry or Submission through the development process.

Statuses should follow these guidelines:

- Statuses should generally be changed as the result of an explicit user action.

- Statuses should generally not restrict functionality of the PR3. Functionality changes based on status should be reversible. (For example, some statuses should default to read-only mode but not restrict access to editing the item.)

- Status changes should always be reversible by the user.

- The user should be able to move from any status to any other status. However, the next and previous statuses in the flow should be obvious to the user (shown at the top of the selection list, in bold, etc.).

- Status values should not attempt to enforce a workflow but should allow users to follow their own (or their Member State’s) workflow.

The goal is to create status flows for both Entries and Submissions that, when combined together, model the ways a State may organize its development process. This includes major steps like drafting new data, reviewing data for completeness, revising data (as a result of a rejection during review), preparing completed Entries for submission, and responding to Agency feedback to a Submission. 


\subsubsection{Uses of Status}

An Entry or Submission's status must accomplish two goals:

- tell the user, at a glance, what action is expected to be taken next on an Entry or Submission, and

- allow users to filter out items they have completed or items still awaiting their action.

The Entry and Submission Statuses in this section are designed such that any role (as identified in the SRS, Section 3.1) will have certain statuses representing incoming or outgoing items. (Note that a user may fill more than one role, and PR3 cannot determine which role(s) a user fills in the process.)

Incoming statuses represent items received from another user or items that are still in progress. A user functioning in this role may filter on these statuses to determine which items are still awaiting his or her action.

Outgoing statuses represent items that have been completed and (depending on the status) sent to another user or to the Agency. A user functioning in this role may filter out these statuses to ignore items not awaiting his or her action (Fig. 44 and Table 7).

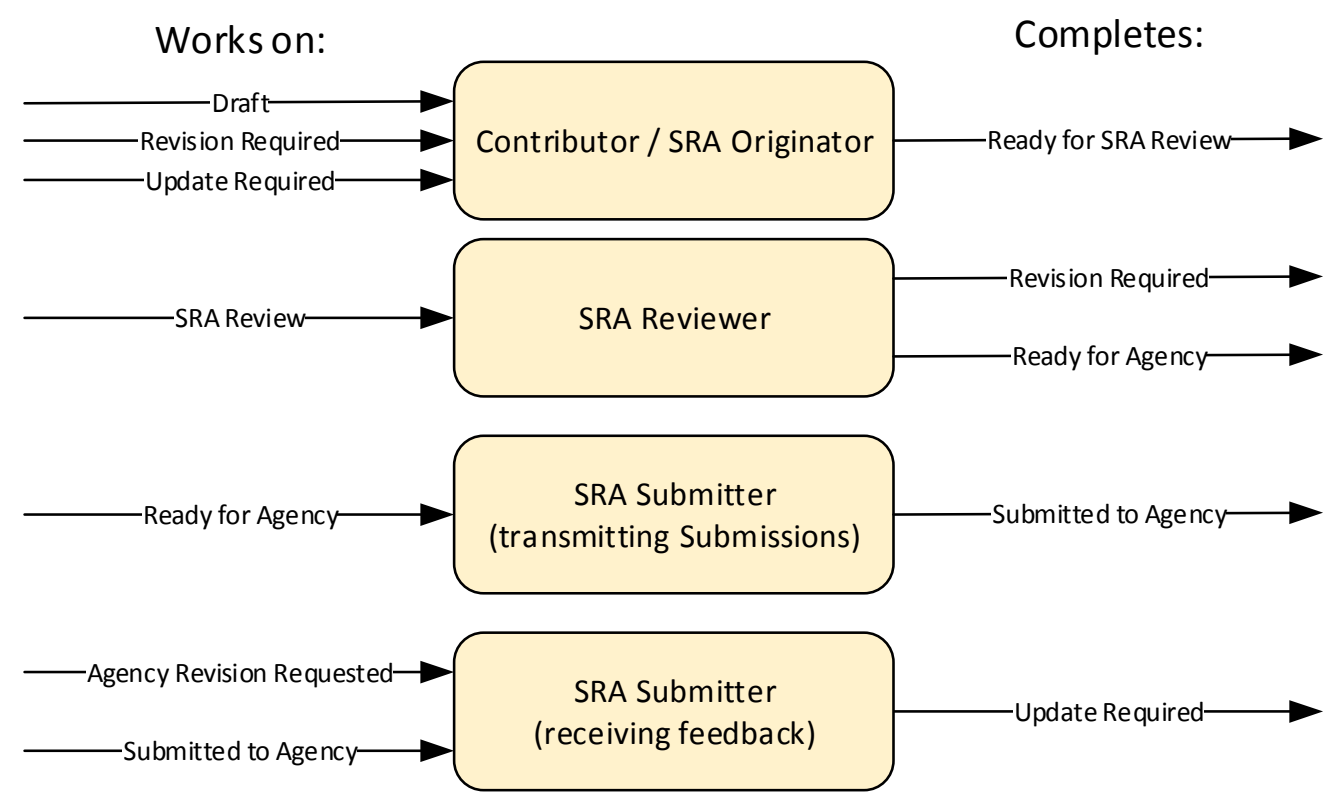

Fig. 44. The roles users may play in a PR3 workflow and what statuses represent to those roles. 
Table 7. The roles users may play in a PR3 workflow and what statuses represent to those roles

\begin{tabular}{|c|c|c|c|}
\hline Role & Description & Incoming Statuses & Outgoing Statuses \\
\hline $\begin{array}{l}\text { Contributor / } \\
\text { SRA Originator }\end{array}$ & $\begin{array}{l}\text { Field subject matter expert who } \\
\text { creates and edits Entries }\end{array}$ & $\begin{array}{l}\text { Draft } \\
\text { Revision Required / } \\
\text { Agency Revision } \\
\text { Requested } \\
\text { Update Required }\end{array}$ & Ready for SRA Review \\
\hline SRA Reviewer & $\begin{array}{l}\text { SRA subject matter expert who } \\
\text { reviews Entries and } \\
\text { Submissions }\end{array}$ & SRA Review & $\begin{array}{l}\text { Ready for Agency (if approved) } \\
\text { Revision Required (if rejected) }\end{array}$ \\
\hline & $\begin{array}{l}\text { Finalizes and transmits } \\
\text { Submissions to the Agency }\end{array}$ & $\begin{array}{l}\text { Ready for Agency (for } \\
\text { transmission) }\end{array}$ & Submitted to Agency \\
\hline SRA Submitter & $\begin{array}{l}\text { Receives feedback from the } \\
\text { Agency about a Submission }\end{array}$ & $\begin{array}{l}\text { Submitted to } \\
\text { Agency(acceptance) } \\
\text { Agency Revision } \\
\text { Requested (information } \\
\text { request) }\end{array}$ & $\begin{array}{l}\text { Update Required (Previously } \\
\text { submitted items that need update) } \\
\text { Agency Revision Requested (sent } \\
\text { back through revision process) }\end{array}$ \\
\hline
\end{tabular}

\subsubsection{Entry Status}

The Entry Status flow must accommodate the following workflow choices:

- Some States may create Entries at one or more Contributors as well as the SRA; others may create Entries only at the SRA.

- Some States may enforce a Review process at the Contributor level, the SRA level, or both.

To accomplish this, the Entry status flow has Draft and Review statuses (Fig. 45 and Table 8). It differentiates between the Contributor and SRA-level Review because an Entry may move from Review on one level to the other. However, an Entry does not have to go through all of these phases during its life cycle; they are available in case the user wants to use them. 


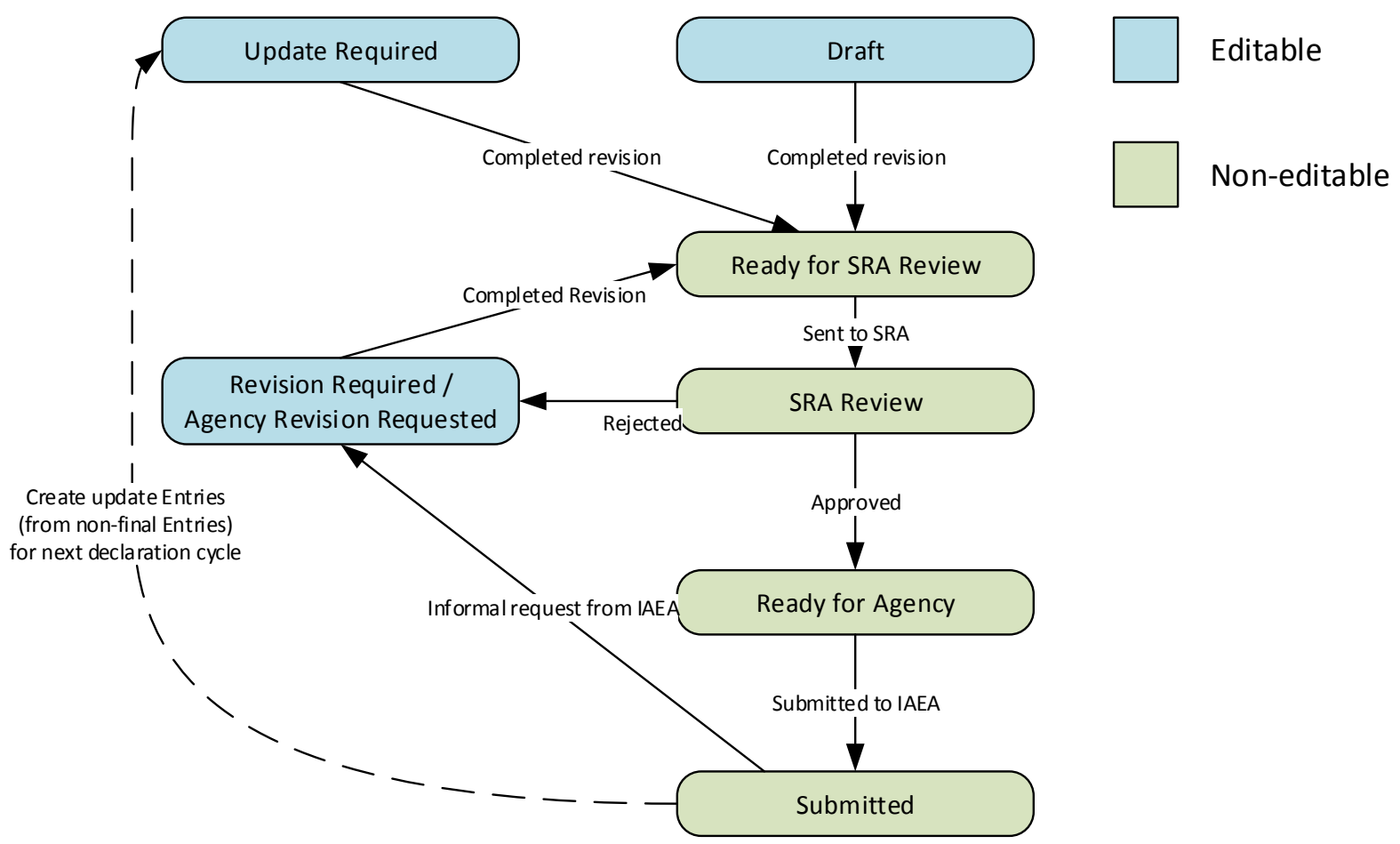

Fig. 45. A complete workflow that uses all available Entry Statuses.

Table 8. Available Entry Statuses, their purpose, and their place within a typical workflow

\begin{tabular}{|c|c|c|}
\hline Status & Purpose & Actions \\
\hline Draft & $\begin{array}{l}\text { The Entry is being edited, } \\
\text { either at the Contributor or } \\
\text { SRA level. }\end{array}$ & $\begin{array}{l}\text { The user may edit all values in the Entry. } \\
\text { This is the default Status value for a new Entry. The next } \\
\text { status value should be Ready for SRA Review. }\end{array}$ \\
\hline $\begin{array}{l}\text { Ready for SRA } \\
\text { Review }\end{array}$ & $\begin{array}{l}\text { The SRA or Contributor } \\
\text { Originator is finished making } \\
\text { revisions to the Entry but has } \\
\text { not yet sent it for SRA } \\
\text { Review. }\end{array}$ & $\begin{array}{l}\text { The user may not edit any values in the Entry except } \\
\text { Status. } \\
\text { The next status value should be SRA Review, which } \\
\text { should be set when the Entry is submitted to the SRA } \\
\text { review process. }\end{array}$ \\
\hline SRA Review & $\begin{array}{l}\text { The Entry is being reviewed at } \\
\text { the SRA level and/or } \\
\text { consolidated into a } \\
\text { Submission. }\end{array}$ & $\begin{array}{l}\text { The user may not edit any values in the Entry except } \\
\text { Status. } \\
\text { The next status value should be SRA Approved if } \\
\text { approved or Revision Required if rejected. }\end{array}$ \\
\hline Revision Required & $\begin{array}{l}\text { The Entry has failed review } \\
\text { and is being edited either at } \\
\text { the Contributor or SRA level. }\end{array}$ & $\begin{array}{l}\text { The user may edit all values in the Entry. } \\
\text { The next status value should be Ready for SRA Review. }\end{array}$ \\
\hline Ready for Agency & $\begin{array}{l}\text { The Entry has been approved } \\
\text { at the SRA level and/or } \\
\text { consolidated into a } \\
\text { Submission. }\end{array}$ & $\begin{array}{l}\text { The user may not edit any values in the Entry except } \\
\text { Status. } \\
\text { The next status value should be Submitted to Agency. }\end{array}$ \\
\hline
\end{tabular}


Table 8. Available Entry Statuses, their purpose, and their place within a typical workflow (continued)

\begin{tabular}{|c|c|c|}
\hline Status & Purpose & Actions \\
\hline \multirow{3}{*}{ Submitted to Agency } & \multirow{3}{*}{$\begin{array}{l}\text { The Entry has been Submitted } \\
\text { to the Agency as part of a } \\
\text { Submission. }\end{array}$} & $\begin{array}{l}\text { The user may not edit any values in the Entry except } \\
\text { Status. }\end{array}$ \\
\hline & & $\begin{array}{l}\text { This status may be set automatically for all Entries within } \\
\text { a Submission exported for transfer to the Agency, or } \\
\text { manually by the user. }\end{array}$ \\
\hline & & $\begin{array}{l}\text { A user may still change the status of a Submitted Entry } \\
\text { back to any other status. }\end{array}$ \\
\hline \multirow{2}{*}{$\begin{array}{l}\text { Agency Revision } \\
\text { Requested }\end{array}$} & \multirow{2}{*}{$\begin{array}{l}\text { The Agency has requested an } \\
\text { informal revision to the Entry. }\end{array}$} & $\begin{array}{l}\text { The user may edit all values in the Entry. The Entry } \\
\text { behaves exactly like a Revision Required Entry. }\end{array}$ \\
\hline & & $\begin{array}{l}\text { The next status value should be Ready for SRA Review } \\
\text { (as the review process should be repeated). }\end{array}$ \\
\hline \multirow[b]{2}{*}{ Update Required } & \multirow{2}{*}{$\begin{array}{l}\text { The Entry has been created to } \\
\text { update a previously submitted } \\
\text { Entry and is being edited, } \\
\text { either at the Contributor or } \\
\text { SRA level. }\end{array}$} & The user may edit all values in the Entry. \\
\hline & & The next status value should be Ready for SRA Review. \\
\hline \multirow[b]{2}{*}{ Cancelled } & $\begin{array}{l}\text { The Entry will not be } \\
\text { developed further or }\end{array}$ & The user may not edit any values in the Entry. \\
\hline & $\begin{array}{l}\text { Submitted to the Agency. } \\
\text { However, it is being kept for } \\
\text { reference. }\end{array}$ & $\begin{array}{l}\text { An Entry's Status may be changed from any other status to } \\
\text { Cancelled, and vice versa. }\end{array}$ \\
\hline
\end{tabular}

\subsubsection{Submission Status}

The Submission Status flow will be simpler because Submissions are assumed to be created and edited only at the SRA level. It is possible for Contributors to create Submissions, but this workflow is not reflected in Status values as this is not a typical case.

The Submission Status flow is built to accommodate a workflow where a Submission is drafted and then reviewed separately before submission to the Agency. A state can use either entry statuses or submission statuses, or both, depending on the needs of its workflows (Fig. 46 and Table 9).

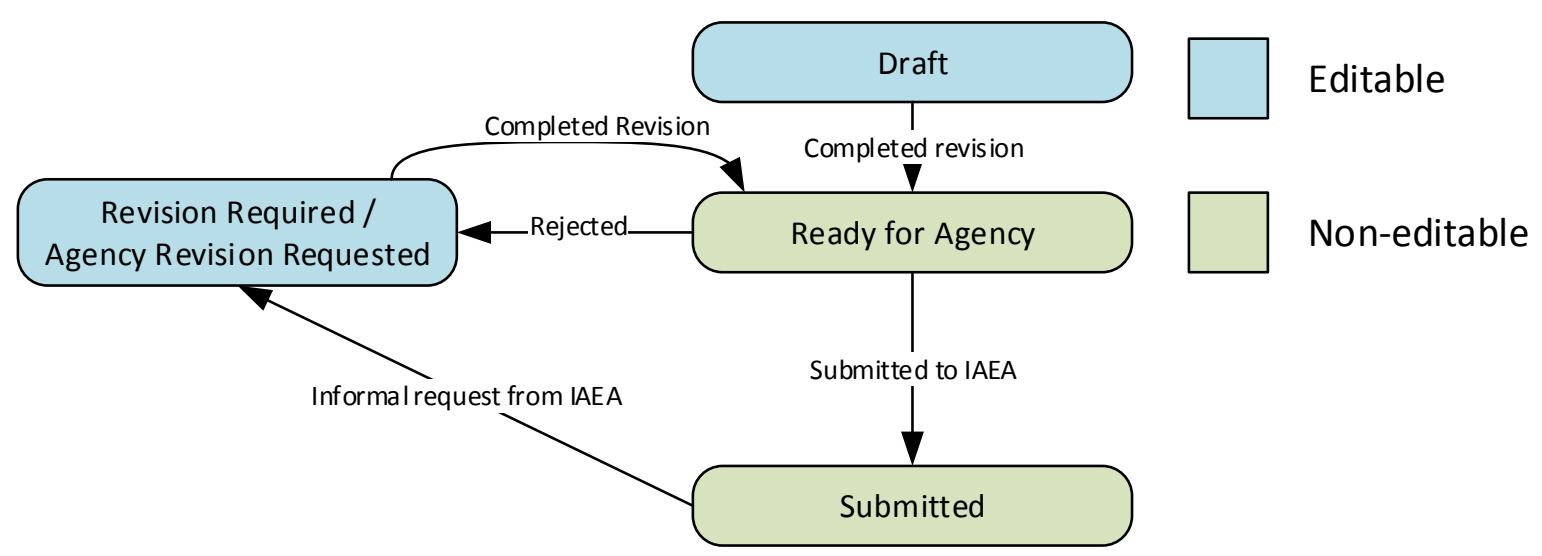

Fig. 46. A complete workflow that uses all available Submission Statuses. 
Table 9. Available Submission Statuses, their purpose, and their place within a typical workflow

\begin{tabular}{|c|c|c|}
\hline Status & Purpose & Actions \\
\hline Draft & $\begin{array}{l}\text { The Submission is being } \\
\text { edited. }\end{array}$ & $\begin{array}{l}\text { The user may edit all values in the Submission. } \\
\text { This is the default Status value for a new Submission. The } \\
\text { next status value should be Ready for Agency. }\end{array}$ \\
\hline Revision Required & $\begin{array}{l}\text { The Submission has failed } \\
\text { review and is being edited. }\end{array}$ & $\begin{array}{l}\text { The user may edit all values in the Submission. } \\
\text { The next status value should be Ready for Agency. }\end{array}$ \\
\hline Ready for Agency & $\begin{array}{l}\text { The Submission has been } \\
\text { approved. }\end{array}$ & $\begin{array}{l}\text { The user may not edit any values in the Submission except } \\
\text { Status. } \\
\text { The next status value should be Submitted to Agency or } \\
\text { Revision Required, depending on whether any revisions } \\
\text { are needed before Submission. }\end{array}$ \\
\hline Submitted to Agency & $\begin{array}{l}\text { The Submission has been } \\
\text { Submitted to the Agency. }\end{array}$ & $\begin{array}{l}\text { The user may not edit any values in the Submission except } \\
\text { Status. } \\
\text { This status may be set automatically when a Submission is } \\
\text { exported for transfer to the Agency, or manually by the } \\
\text { user. }\end{array}$ \\
\hline & & $\begin{array}{l}\text { A user may still change the status of a Submitted } \\
\text { Submission back to any other status. }\end{array}$ \\
\hline $\begin{array}{l}\text { Agency Revision } \\
\text { Requested }\end{array}$ & $\begin{array}{l}\text { The Agency has requested an } \\
\text { informal revision to the } \\
\text { Submission. }\end{array}$ & $\begin{array}{l}\text { The user may edit all values in the Submission. The } \\
\text { Submission behaves exactly like a Revision Required } \\
\text { Submission. } \\
\text { The next status value should be Ready for Agency. }\end{array}$ \\
\hline
\end{tabular}

\subsubsection{Comparison to the PR2}

PR2 implemented a workflow for developing Submissions by using three folders: Imported Declarations, Working Declarations, and Submitted Declarations. The available PR3 Submission Status values are intended to be more granular versions of these statuses such that each PR3 status value can map directly to a single PR2 status (Table 10). One major difference is that PR3 statuses can apply to individual entries while PR2 statuses applied to submissions and all of their associated entries. 
Table 10. Comparison of PR2 Statuses to PR3 Statuses

\begin{tabular}{|c|c|c|}
\hline PR2 Status & Equivalent PR3 Statuses & Notes \\
\hline Imported & (None) & $\begin{array}{l}\text { The "Imported Declarations" folder represents a holding } \\
\text { area for newly imported Submissions. These Submissions } \\
\text { are not editable and must be moved to the Working folder. } \\
\text { PR3 would import this data directly. An Entry's or } \\
\text { Submission's Status would be preserved during export and } \\
\text { import, so the receiving user would not have to decide how } \\
\text { to categorize it after import. (However, the user could } \\
\text { choose to change the status during the import process.) }\end{array}$ \\
\hline Working & $\begin{array}{l}\text { Draft } \\
\text { Ready for SRA Review } \\
\text { SRA Review } \\
\text { Revision Required } \\
\text { Ready for Agency } \\
\text { Update Required } \\
\text { Agency Revision Requested }\end{array}$ & $\begin{array}{l}\text { The "Working Declarations" folder represents in-progress } \\
\text { Submissions that can be edited. PR3 adds additional } \\
\text { granularity to this status. }\end{array}$ \\
\hline Submitted & Submitted to Agency & $\begin{array}{l}\text { The "Submitted Declarations" folder represents } \\
\text { Submissions that have been validated, can be exported to } \\
\text { Agency XML format and are no longer editable. } \\
\text { Because a PR2 Submission can only be exported if it is in } \\
\text { this folder, "Submitted" may be a misnomer. For example, } \\
\text { a Contributor's "Submitted" folder will likely include } \\
\text { Entries that have been sent to another Contributor or the } \\
\text { SRA for review but not yet submitted to the Agency. }\end{array}$ \\
\hline N/A & Cancelled & $\begin{array}{l}\text { Applies to Entries that remain in the user's database but } \\
\text { will not be subject to further processing or submission. }\end{array}$ \\
\hline
\end{tabular}

\subsection{ENTRY AND DECLARATION TYPE WORKFLOW}

The purpose of this section is to define how Entry and Declaration types may be used to represent the history of declaration data over the course of multiple Submissions. It is intended to be a guide for developers to understand how the Type fields provide information to the Agency about the history of declaration data.

\subsubsection{Overview}

PR3 is designed to assist Member States in developing and organizing their declaration data over the entire life cycle of that data, including key steps such as

- the Member State’s initial declaration,

- regular updates to the initial declaration,

- responses to Agency requests,

- Entries for new activities or locations,

- Entries that serve as updates of previously declared activities or locations, and 
- Entries that indicate an activity or location has been terminated and/or will not be subsequently updated.

To do this, Entries and Declarations have a "Type" field that indicates which of these functions that Entry or Declaration serves.

Unlike the Status field, the Type for a single Entry or Declaration should remain constant throughout its development and review cycle. Status indicates an item's progress through the development and review cycle at a given point in time, and Type indicates the reason an Entry is submitted to the IAEA and where it falls within the history of a location or activity or a where Declaration falls within a Member State's set of AP declarations (Fig. 47).

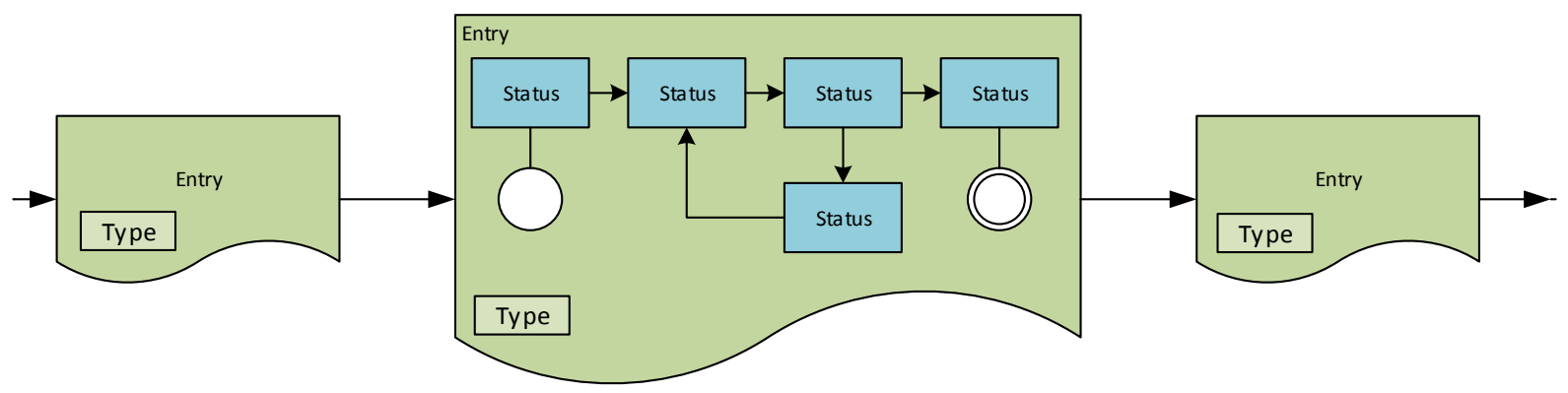

Fig. 47. Comparison of Statuses and Types.

\subsubsection{Entry Type}

An Entry represents a single declarable activity or location for a Member State (Fig. 48 and Table 11). There are two questions the Entry type must answer:

- What does this Entry tell the Agency about the activity or location? Either this is a new activity or location, an update about a previously declared activity or location, a notice that nothing has changed since the last time an activity or location was declared, or a summary of all updates to an activity or location.

- Will this activity or location be updated in the future? If an activity or location has been terminated over the course of the declaration period, then it will not be updated during the next declaration period.

The Entry Type field answers the first question. There is an additional true/false field on Entries named "Final Entry" (see SRS C.1 Base Entry Data Structure) that may be used in conjunction with the "New entry" and "Revised entry" types to answer the second question.

There should be two restrictions for the Final Entry flag:

- It should not be used with the "No change" Entry Type because an explanation of why the activity or location was terminated should be included.

- It should be hidden on Entries for activities that do not have a defined end or are per-instance entries. For example, it should be possible to mark Model AP 2.a.(i) as Final Entry (R\&D activities that end are no longer reported) but not Model AP 2.a.(v) (as a mine that is no longer in use continues to be reported as abandoned). Likewise, Model AP 2.a.(ix) entries for imports and exports are reported on a per-instance basis and are not subject to update, so they need never be reported as Final entries. 


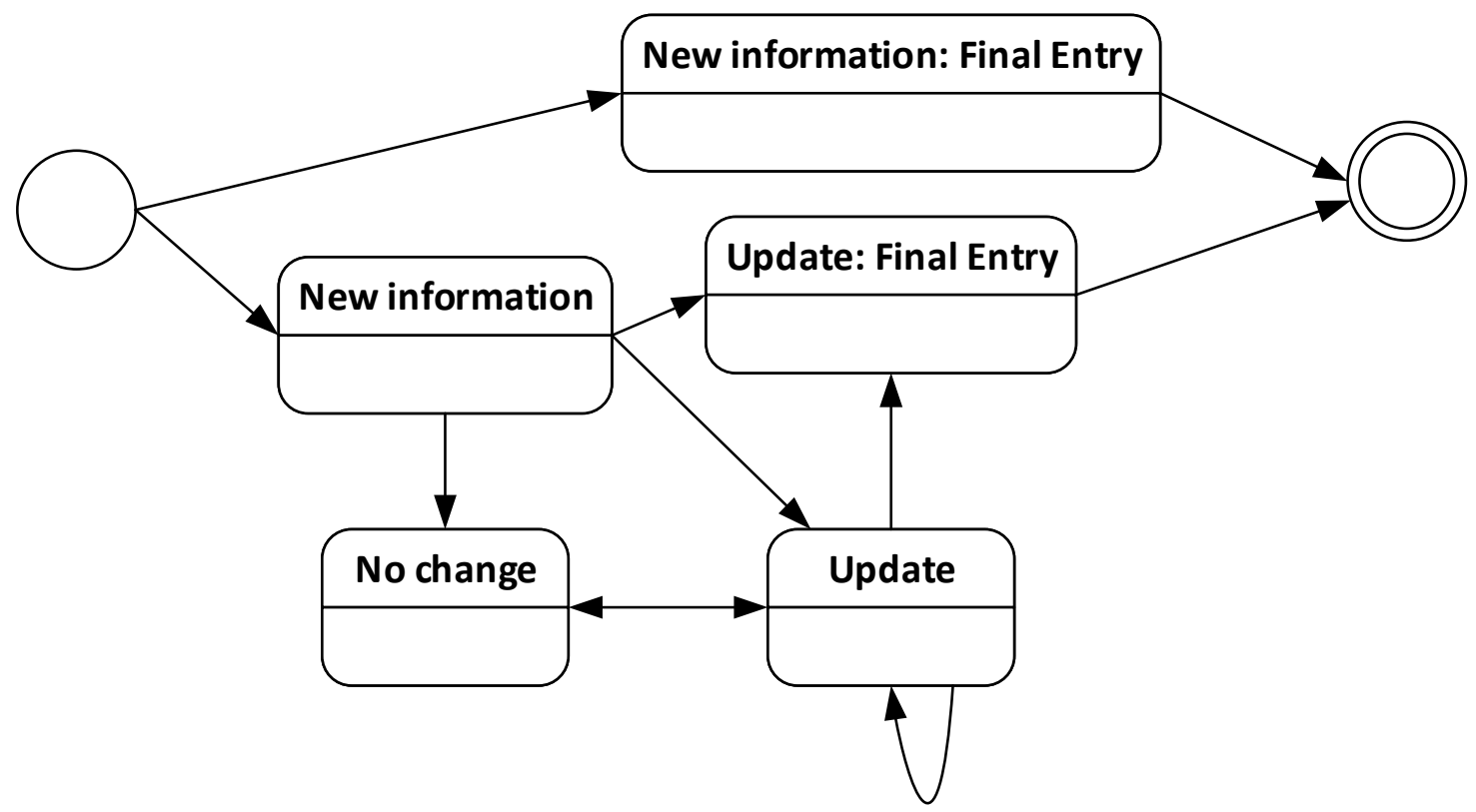

Fig. 48. A state diagram for Entry Types and the Final Entry flag.

Table 11. The five valid combinations of Entry Type and Final Entry

\begin{tabular}{|c|c|c|c|}
\hline State & Entry Type field & Final Entry field & Meaning \\
\hline New information & New information & No & $\begin{array}{l}\text { This is the first time this activity or location has } \\
\text { been declared. If applicable for the AP article, it } \\
\text { will be updated as part of the next update } \\
\text { declaration. }\end{array}$ \\
\hline Update & Update & No & $\begin{array}{l}\text { This is an update to an activity or location that } \\
\text { has previously been declared. This Entry should } \\
\text { include a reference to the previous Entry. }\end{array}$ \\
\hline No change & No change & No & $\begin{array}{l}\text { A previously declared activity or location has not } \\
\text { changed since it was last reported. This Entry } \\
\text { should include a reference to the previous Entry. }\end{array}$ \\
\hline $\begin{array}{l}\text { New information: } \\
\text { Final Entry }\end{array}$ & New information & Yes & $\begin{array}{l}\text { This is the first time this activity or location has } \\
\text { been declared. It will not be updated in future } \\
\text { update declarations. }\end{array}$ \\
\hline Update: Final Entry & Update & Yes & $\begin{array}{l}\text { This is an update to an activity or location that } \\
\text { has previously been declared. It will not be } \\
\text { updated in future update declarations. }\end{array}$ \\
\hline
\end{tabular}

Note that marking an Entry as Final Entry is only an indication to the Agency that updates to the activity or location should not be expected in the future. This will not prevent that Entry from being updated in the future should it become necessary.

\subsubsection{Declaration Type}

Unlike the Entry Type field, the Declaration Type field (Fig. 49 and Table 12) must answer only one question: what does this Declaration tell the Agency about the activity or location? Either there is new declarable information to report about a particular AP article, there is nothing to declare under a particular 
AP article, or the information about activities and locations declarable under a particular AP article has not changed since the last time it was declared.

Because a Declaration is a collection of Entries and is considered less specific than an Entry, there are a few differences between Entry Type and Declaration Type:

- Revised or updated Declarations: Whether Entries represent new or previously declared information, the Declaration that contains them is always considered "new information." This is simpler than requiring users to differentiate between Declarations that contain all new Entries, all updated Entries, or some combination of new and updated Entries.

- Final Entry: A Declaration may contain Final Entries, but there is no equivalent status for Declarations. A Member State is always responsible for submitting a declaration for each applicable AP article even if there is nothing to declare under a given article.

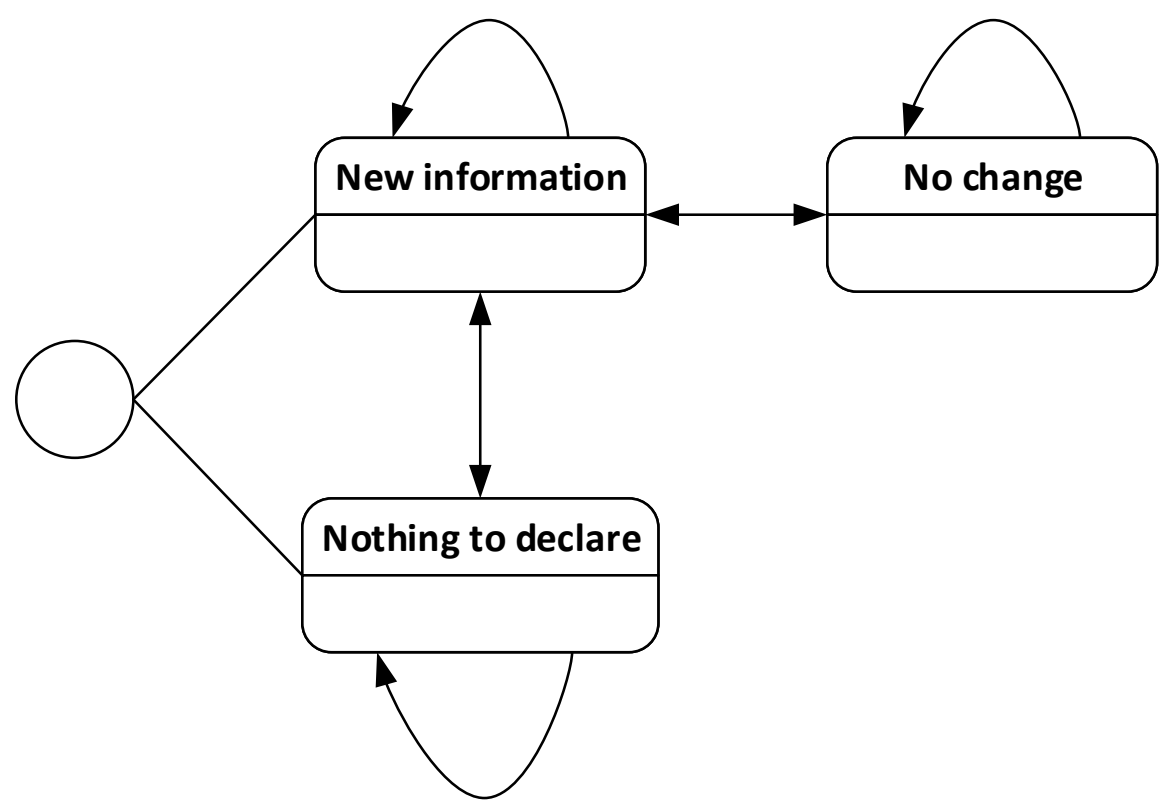

Fig. 49. A state diagram for Declaration Types.

Table 12. The three Declaration Types

\begin{tabular}{ll}
\hline \multicolumn{1}{c}{ Declaration Type } & \multicolumn{1}{c}{ Meaning } \\
\hline New information & $\begin{array}{l}\text { This Declaration contains Entries representing new and/or revised information about } \\
\text { activities and locations declarable under this AP article. }\end{array}$ \\
Nothing to declare & $\begin{array}{l}\text { There are no activities or locations that are declarable under this AP article. This } \\
\text { Declaration should not contain any Entries. }\end{array}$ \\
No change & $\begin{array}{l}\text { No information has been added, modified, or deleted regarding the activities and location } \\
\text { in this Declaration since the last time they were submitted. This Declaration must contain } \\
\text { only No Change Entries. }\end{array}$ \\
\hline
\end{tabular}




\subsection{NUMBERING RULES}

\subsubsection{Entry Numbering Rules}

By default, all Entries within a Declaration are numbered in the order in which they are added to the Declaration. Numbering occurs sequentially from top to bottom in the list of entries as they appear in the selected declaration, starting at 1 .

Renumbering occurs whenever one of these actions is taken in the Submission Editor:

- An Entry or Entries are added to the Declaration. Automatic renumbering will occur based on the existing Entry numbering, with the new Entry or Entries at the end of the list.

- An Entry is removed from the Declaration. Automatic renumbering will occur based on the existing Entry numbering.

- The user chooses to renumber the Declaration based on Entry sort order. Renumbering will occur based on the current sort order of the Entries list in the Submission Editor.

When an Entry is renumbered, any cross-references to that Entry in the user's database should be updated if possible.

Although Declarations are automatically renumbered based on their order, Entries are not. This allows Entries to be sorted or filtered within the grid view for ease of organization.

\subsubsection{Declaration Numbering Rules}

All Declarations within a Submission are automatically numbered by declaration order. Numbering occurs sequentially starting at 1 unless a different starting number is chosen for the Submission. (When editing the starting number for a Submission, the PR3 will suggest a number based on the last Submission to be exported for Agency transmission.)

This process occurs whenever one of these actions is taken in the Submission Editor:

- A new Declaration is added

- A Declaration is deleted

- A Declaration is moved up or down in the Declaration list

- A new starting number is chosen for the Submission

When a Declaration is renumbered, any cross-references to that Declaration or its Entries in the user's database should be updated if possible.

\subsection{GUIDED WIZARDS}

Wherever possible, the PR3 should provide the user with "wizards" that automate common processes that would otherwise require specific AP knowledge. There are two main objectives to this approach:

- Ease new users into the Entry or Submission development process and prevent them from being overwhelmed by the granularity of the forms. 
- Encourage all users to follow the ideal workflows for Entry and Submission development by making it the easiest possible choice.

Depending on the wizard, it may also be possible for advanced users to perform the same functions manually.

Most wizards will contain questions or answer choices that vary between data templates. For this reason, the PR3 must provide an interface for supplying this information. If a plugin does not implement these interfaces, then the corresponding wizards must be disabled for that data template.

\subsubsection{New Entry Wizard}

The New Entry Wizard is intended to guide Contributors through creating the proper type of Entry and filling in identifying information. Its objective is to abstract away the AP-specific parts of the process, allowing the user to focus on documenting the activity or location.

As a user becomes more familiar with creating Entries through using the wizard, it should become possible for him or her to start doing so manually.

The New Entry Wizard asks the following questions, in order:

\section{What state or entity will report this activity to the IAEA?}

\section{Answer Choices}

Each answer choice corresponds to an installed data template. State- or entity-specific data templates will be displayed as their name. The Model AP template will be shown at the end of the list as "A State or Entity not listed here.”

\section{Purpose}

The answer to this question will determine what data template is used for the Entry. It will also affect the choices in step 2.

\section{Requirements}

The user must select one choice to move to the next step.

If only one data template is installed, it will be selected by default, and the user will skip to step 2 automatically.

\section{What is the nature of the activity?}

\section{Answer Choices}

Each choice corresponds to an Entry article for the data template selected in step 1.

For users with little or no AP knowledge, choices should be labeled by purpose ("Government-sponsored NFC R\&D not involving nuclear material”) rather than article [“2.a.(i)”].

To limit the number of choices a user has to consider, choices should be grouped by type of activity ("R\&D," "Materials and Waste," "Operations," etc.). Note that some article types may fit under multiple groupings and should be shown wherever applicable to help the user find the correct activity.

A data template plugin is responsible for providing these groupings and labels for this step in the wizard. 


\section{Purpose}

The answer to this question will determine what Entry article is used for the new Entry.

\section{Requirement}

The user must select one choice to move to the next step.

If a user goes back to step 1 and selects a different value, he or she will see a different set of choices upon returning to step 2.

Examples of these choices for the Model AP might be the following:

- $\mathrm{R} \& \mathrm{D}$

○ Government-sponsored nuclear fuel cycle R\&D not involving nuclear material [Article 2.a.(i)]

- Privately sponsored nuclear fuel cycle R\&D not involving nuclear material [Article 2.b.(i)]

○ Nuclear fuel cycle R\&D 10-year plan [Article 2.a.(x)]

- Materials or Waste

○ Source material holdings [Article 2.a.(vi)(a)]

- Source material export [Article 2.a.(vi)(b)]

- Source material import [Article 2.a.(vi)(c)]

- Nuclear material exempted from safeguards [Article 2.a.(vii)]

○ Change in location of intermediate- or high-level waste [Article 2.a.(viii) Location Change]

○ Further processing of intermediate- or high-level waste [Article 2.a.(viii) Processing]

- Operations

$\circ \quad$ Building on a site [Article 2.a.(iii)]

- Manufacturing, assembly, or construction activity listed in Annex I [Article 2.a.(iv)]

- Mine or concentration plant production [Article 2.a.(v) Mine/Plant]

○ State production total for mining or concentration [Article 2.a.(v) State Total]

○ Further processing of intermediate- or high-level waste [Article 2.a.(viii) Processing]

- Import or Export

○ Source material export [Article 2.a.(vi)(b)]

○ Source material import [Article 2.a.(vi)(c)]

○ Export of equipment or non-nuclear material listed in Annex II [Article 2.a.(ix)(a)]

○ Import of equipment or non-nuclear material listed in Annex II [Article 2.a.(ix)(b)]

- Agency Requests or Agreements

○ Information agreed with the Agency to facilitate safeguards implementation [Article 2.a.(ii)]

- Operational activities at a location identified by the Agency [Article 2.b.(ii)]

$\circ$ Response to Agency request [Article 2.c]

\section{How will this activity be reported to the IAEA?}

Answer Choices

"As part of an initial submission, "As part of an annual submission," "As part of a quarterly submission," "As a response to an agency request," or "I don't know."

\section{Purpose}

The answer to this question will determine how the user is prompted for the reporting period in the next step. The Entry's Reporting Period will be set based on this value: 
- As part of an initial submission: Initial

- As part of an annual submission: Annual

- As part of a quarterly submission: Quarterly

- As a response to an agency request: Date

- I don't know: the Reporting Period will be left blank

\section{Requirement}

The user must select one choice to move to the next step.

\section{What time period will this entry cover?}

\section{Prompt}

The prompt the user is shown will depend on his or her answer to step 3:

- As part of an initial submission: The user will be prompted for an As-Of Date with a date picker control (which will be set as the Entry's Reporting Period Start and End dates if provided). This will be pre-populated with the Default As-Of Date from Settings.

- As part of an annual or quarterly submission: The user will be prompted for the Reporting Period Start and End dates with a date picker control. This will be pre-populated with the last saved Entry's reporting period.

- As a response to an agency request: The user will be prompted to select the Agency Request Date with a date picker control.

- I don't know: The user will skip this step.

\section{Purpose}

Along with step 3, this question will determine what the Entry's reporting period will be.

\section{Requirement}

The user may skip this step without selecting or entering a value, in which case the corresponding Reporting Period fields will be left blank (to be filled in later).

\section{Has this activity been reported to the IAEA before?}

\section{Answer Choices}

"No, it is new information," "Yes, and it has changed," or "Yes, and it has not changed."

\section{Purpose}

The answer to this question will set the Entry Type field:

- No, it is new information: New information

- Yes, and it has changed: Update

- Yes, and it has not changed: No change

\section{Requirement}

The user must select one choice to move to the next step. 


\section{Will this activity be reported again?}

Answer Choices

"Yes, it is still ongoing” or "No, it concluded during this reporting period."

\section{Purpose}

The Entry's Final Entry field will be set if the user selected "No, it concluded during this reporting period."

\section{Requirement}

The user must select one choice to move to the next step.

\section{Confirm and Finish}

When the user closes the wizard, the Entry Editor will be opened containing the new Entry. The data template and article will be chosen, and the Entry Type, Reporting Period, and Final Entry fields will be populated.

\subsubsection{Prepare Update Entries Wizard}

The Prepare Update Entries Wizard is intended to guide a user at the SRA through creating Entries to update previously submitted data. A Contributor who had copies of Entries he or she previously submitted to the SRA could also use this wizard if his/her SRA chose not to do so.

It has two objectives:

- Ensure that a declaration meant to update previously declared activities (e.g., a Model AP 3.b) contains an Entry to update each applicable ongoing activity or location (even if it signifies no change to that activity or location).

- Ensure that each update Entry is a consolidated update containing a full description of that activity or location.

Using the Prepare Update Entries wizard should produce both higher-quality updates for the Agency and be easier for the State than creating annual or quarterly update declarations manually.

The Prepare Update Entries wizard asks the following questions, in order:

\section{What state or entity will report this activity to the IAEA?}

\section{Answer Choices}

Each answer choice corresponds to an installed data template. State- or entity-specific data templates will be displayed as their name. The Model AP template will be shown at the end of the list as "A State or Entity not listed here.”

\section{Purpose}

The answer to this question will determine what data template is used for the Entries or Submission created by the wizard. It will also affect the choices in step 2 .

\section{Requirements}

The user must select one choice to move to the next step. 
If only one data template is installed, it will be selected by default, and the user will skip to step 2 automatically.

\section{What are you updating?}

\section{Answer Choices}

Each choice corresponds to a Submission type for the data template selected in step 2.

For users with little or no AP knowledge, choices should be labeled primarily by purpose, with references to articles for clarification. For example, in the Model AP template, "The initial Submission (3.a) or annual update Submission (3.b) for this State” would be an option to create a 3.b Submission.

A data template plugin is responsible for providing these groupings and labels for this step in the wizard.

\section{Purpose}

The answer to this question will determine what Submission article is used for the Entries or Submission created by the wizard. It will also affect the types of Entries shown in step 3.

\section{Requirements}

The user must select one choice to move to the next step.

If a user goes back to step 1 and selects a different value, he or she will see a different set of choices when returning to step 2.

If only one type of Submission is valid for the selected data template, it will be selected by default, and the user will skip to step 3 automatically.

\section{Which Entries need to be updated?}

\section{Prompt}

The user may select one or more Entries from a data grid containing all Entries in the PR3 database that meet the following requirements:

- $\quad$ are associated with the selected data template (from step 1),

- $\quad$ can be included in the Submission type that is being updated (from step 2),

- have a status of Submitted, IAEA - Revision Requested, or IAEA - Receipt Acknowledged,

- have not been updated before, and

- $\quad$ are not marked as Final Entry (the user may optionally remove this filter).

\section{Purpose}

The selected Entries will be used to as the basis for the update Entries created by the wizard.

\section{Requirements}

The user must select one or more Entries to move to the next step. If the user selects any Entries that are Final Entry for update, a warning and confirmation prompt should be shown.

\section{How would you like to prepare the Update Entries?}

\section{Answer Choices}

"Create an individual Update Entry for each selected Entry" or "Create a Submission containing the Update Entries for all selected Entries.” 


\section{Purpose}

The answer will determine how Entries will be created (see Confirm and Finish for details), which depends on the user's desired workflow.

\section{Requirements}

The user must select one choice to move to the next step.

\section{Confirm and Finish}

When the user closes the wizard, one of two things will happen depending on the user's answer in step 4:

- Create an individual Update Entry for each selected Entry: Individual update Entries will be created in the PR3 database. The user will be taken back to the Data Manager, and the view will switch to show in-progress Entries.

- Create a Submission containing the Update Entries for all selected Entries: The Submission Editor will be opened containing a Submission of the selected type (from step 2). The Submission will contain the update Entries organized into their respective Declarations.

Each update Entry will be created with a copy of the data from one of the selected Entries. A new internal ID will be assigned, the reporting period will be updated (based on the last Submission in the PR3 database for the selected data template), and the Entry Type will be set to Update. The old Entry and new Entries will be linked in the database for future reference.

\subsection{RULES FOR DEFAULT VALUES}

To ensure simplicity and accuracy, PR3 will fill in default values in new Entries, Declarations, and Submissions whenever it is possible to determine them.

In most cases, PR3 settings or previous Submissions stored in the database will be used as the basis for these default values. Particular rules will be used to determine what the actual values should be; for example, rules will define how a previous Submission's reporting period can be used to calculate the next Submission's reporting period. In some cases, these rules may need to be implemented in data template plugins rather than PR3.

When implementing rules for default values, two guidelines must be considered:

- If a value needed to pre-populate a field with a default value is unavailable, that field should be left blank.

- If the rules for pre-populating a field with a default value may be unreliable, that field should be left blank or the user should be warned to check the value.

Support for these rules may need to be included in data templates.

\subsubsection{Initial Declaration As-Of Date}

When a new Declaration is created in an initial submission (3.a in the Model AP, but this may be different for other templates), the reporting period date should be pre-populated as follows:

1. If another Declaration exists in the same Submission, then the last created Declaration's reporting period date will be used. 
2. If no other Declarations exist in the same Submission, the default As-Of Date from Settings will be used.

3. If the As-Of Date in Settings is blank, the Entry Into Force date (from either the data template or Settings) will be used.

4. If none of the other conditions are fulfilled, the date will be left blank.

\subsubsection{Annual Update Reporting Period}

When a Declaration is created in an annual submission (3.b, 3.c, or 3.e in the Model AP, but this may be different for other templates), the reporting period date will be pre-populated as follows:

1. If another Declaration exists in the same Submission, then the last created Declaration's reporting period date will be used.

2. If no other Declarations exist in the same Submission, then PR3 will check the database for a Submission that matches the following criteria:

a. Have a Status of Submitted, IAEA - Receipt Acknowledged, IAEA - Revision Required, or Superseded.

b. Represent a previous Submission of this data (Model AP: for a 3.b Submission, 3.a or 3.b; for a 3.c Submission, 3.c; for a 3.e Submission, 3.e)

c. Have the most recent Submission Date out of any Submissions that match the previous criteria.

3. If step 2 fails, then PR3 will check the database for a Submission that matches the following criteria:

a. Have a Status of Submitted, IAEA - Receipt Acknowledged, or IAEA - Revision Required.

b. Represent any previous annual Submission of data (Model AP: 3.a, 3.b, 3.c, or 3.e).

c. Have the most recent Submission Date out of any Submissions that match the previous criteria.

4. If a Submission is found in step 2 or 3, PR3 will use the following:

a. If the Submission has a Declaration of the same type, add 1 year to that Declaration's reporting period dates (the data template plugin may have to adjust dates if, for example, the previous Submission was an initial).

b. If the Submission does not have a Declaration of the same type, add 1 year to the first Declaration's reporting period dates (the data template plugin may have to adjust dates if, for example, the previous Submission was an initial).

5. If none of the other conditions apply, the reporting period will be left blank.

\subsubsection{Quarterly Reporting Period}

When a Declaration is created for a quarterly Submission (3.d in the Model AP, but this may be different for other templates), the reporting period date will be pre-populated as follows: 
1. If another Declaration exists in the same Submission, then the last created Declaration's reporting period date will be used.

2. If no other Declarations exist in the same Submission, then PR3 will check the database for a Submission that matches the following criteria:

a. Have a Status of Submitted, IAEA - Receipt Acknowledged, IAEA - Revision Required, or Superseded.

b. Are a previous Submission of the same type (Model AP: for a new 3.d Submission, a previous 3.d Submission)

c. Have the most recent Submission Date out of any Submissions that match the previous criteria.

3. If step 2 fails, then PR3 will check the database for a Submission that matches the following criteria:

a. Have a Status of Submitted, IAEA - Receipt Acknowledged, IAEA - Revision Required, or Superseded.

b. Represent any previous quarterly Submission of data. (The Model AP only has 3.d as a quarterly Submission, but other data templates could have more than one quarterly Submission type.)

c. Have the most recent Submission Date out of any Submissions that match the previous criteria.

4. If a Submission is found in step 2 or 3, PR3 will use the following:

a. If the Submission has a Declaration of the same type, add 3 months to that Declaration's reporting period dates.

b. If the Submission does not have a Declaration of the same type, add 3 months to the first Declaration's reporting period dates.

c. If the quarterly declaration is the first to the IAEA, leave the date blank.

5. If none of the other conditions apply, the reporting period will be left blank.

\subsubsection{Submission Starting Declaration Number}

To ensure that Declarations are numbered properly across a State's Submissions, the starting declaration number should be pre-populated with the next available number, if possible. The user can always change the starting number if this is incorrect.

When a user creates a Submission, PR3 should query the database for the highest Declaration number within any Submission type for that Data Template.

- If a matching number is found, add 1 to that number and use it as the starting number.

- If no matching number is found, use the Default Starting Number setting.

Note that, depending on the order in which a user creates and deletes Submissions, a Submission could end up with the wrong number. Validation rules should be put in place to ensure that numbering is correct across all Submissions in the database. 


\subsection{DATA VALIDATION RULES}

The purpose of this section is to define all data validation rules that will be used on specific types of data. It provides a guide for developers to implement validation checks and to show helpful validation results to the user.

\subsubsection{Overview}

The following rules cover all validation checks that PR3 should perform on declaration data.

Validation is intended to assist the user in developing a complete and accurate declaration. Aside from export for transmission to the IAEA, validation should never restrict what actions the user can take on an Entry, Declaration, or Submission.

Wherever possible, the controls used in Entry and Submission Editors should prevent the need for validation of basic formats. For example, date fields should only accept valid dates. Date format validation will never be necessary for these fields because data cannot be entered in an invalid format.

When one data type contains another data type, validating the parent object will also validate all child objects. For example, a Submission contains Declarations which contain Entries, so validating a Submission would validate and return results for all of its Declarations, which would in turn validate and return results for all of their Entries.

Messages are grouped into three categories:

- Error: Identifies required data that is omitted or invalid. This should include data that the Agency or SRA needs to identify the Entry, or data that directly relates to a State's AP requirements. Errors should alert the user to a problem, encourage the user to fix it, and prevent export until the problem is fixed. Errors are the only type of validation message that may restrict functionality. A Submission with validation errors cannot be exported for transmission to the Agency. However, errors should never restrict any other type of saving or exporting.

- Warning: Identifies important data that is omitted, invalid, or in conflict with other data but may be intentional. It should also include any validation messages that cannot be definitively confirmed such as validating a reference to an Entry or Declaration number that is not currently located in the user's data repository.

- Info: Represents other optional data that may have been omitted or invalid but is not required to ensure a valid submission. This should include all other declaration data. A user should only pay attention to this level of validation if he or she wants to perform a complete and thorough review of data.

The purpose of these categories is to allow the user to filter validation results to a particular level of detail. Note that the distinctions between these categories are somewhat arbitrary and may be changed as necessary during implementation.

In each table, Condition represents a conflict with a validation rule, Message represents what a user should actually see if the condition is encountered, and Type represents the severity of condition with respect to its potential for negative impact on a submission. 


\subsubsection{Model AP Validation Rules}

\subsubsection{Entry Data Structures}

\section{Entry}

In addition to these validation rules, the rules for the composite Reference and Document data types will be applied.

\begin{tabular}{|l|l|l|}
\hline \multicolumn{1}{|c|}{ Condition } & \multicolumn{1}{|c|}{ Message } & \multicolumn{1}{c|}{ Type } \\
\hline Contributor is blank. & Contributor is missing. & Warning \\
\hline State is blank. & State is missing. & Warning \\
\hline INFCIRC \# is blank. & $\begin{array}{l}\text { The INFCIRC \# associated with this AP declaration is } \\
\text { missing. }\end{array}$ & Warning \\
\hline $\begin{array}{l}\text { Revised or No Change entry without } \\
\text { an Update Reference. }\end{array}$ & $\begin{array}{l}\text { An Update Reference is missing for a Revised or No Change } \\
\text { Entry. }\end{array}$ & Warning \\
\hline Declarable Comments is blank. & Declarable Comments are missing. & Info \\
\hline Local ID is blank. & Local ID is missing. & Warning \\
\hline Entry Title is blank. & Entry Title is missing. & Warning \\
\hline Reporting Period Start is blank. & Reporting Period Start or As-Of Date is missing. & Warning \\
\hline Reporting Period End is blank. & Reporting Period End is missing. & Warning \\
\hline $\begin{array}{l}\text { Reporting Period Start is greater than } \\
\text { Reporting Period End. }\end{array}$ & $\begin{array}{l}\text { The Reporting Period Start date comes after the End date. } \\
\text { The dates may be reversed. }\end{array}$ & Warning \\
\hline Reporting Period is in the future & The Reporting Period Start or End date is in the future. & Warning \\
\hline $\begin{array}{l}\text { Final Entry is True for a No Change } \\
\text { Entry. }\end{array}$ & A No Change Entry should not be marked as a Final Entry. & Error \\
\hline $\begin{array}{l}\text { Final Entry is True but Declarable } \\
\text { Comments have not changed. }\end{array}$ & $\begin{array}{l}\text { Please enter Declaration Comments to explain why this will } \\
\text { be the Final Entry for this activity. }\end{array}$ & Warning \\
\hline
\end{tabular}

\section{Article 2.a.(i) Entry}

In addition to these rules, the rules for the composite Location, Organizational Involvement, and Foreign Collaboration data types will be applied.

\begin{tabular}{|l|l|l|}
\hline \multicolumn{1}{|c|}{ Condition } & \multicolumn{1}{c|}{ Message } & \multicolumn{1}{c|}{ Type } \\
\hline Fuel Cycle Stages is blank. & Fuel Cycle Stages are missing. & Error \\
\hline Locations is blank. & Locations are missing. & Error \\
\hline Project Title is blank. & Project Title is missing. & Info \\
\hline Project ID is blank. & Project ID is missing. & Info \\
\hline $\begin{array}{l}\text { Relationship of Activity to State is } \\
\text { blank. }\end{array}$ & $\begin{array}{l}\text { Relationship of Activity to State is missing. (If this activity is } \\
\text { not related to the State, then it may not be declarable.) }\end{array}$ & Info \\
\hline Description is blank. & Description is missing. & Error \\
\hline Organizations is blank. & Organizations are missing. & Info \\
\hline Objectives is blank. & Objectives are missing. & Info \\
\hline $\begin{array}{l}\text { Degree to which Objectives Met is } \\
\text { blank. }\end{array}$ & Degree to which Objectives Met is missing. & Info \\
\hline Intended Applications is blank. & Intended Applications are missing. & Info \\
\hline Foreign Collaboration is blank. & Foreign Collaborations are missing. & Info \\
\hline Project Start Date & Project Start Date is missing. & Info \\
\hline Project End Date & Project End Date is missing. & Info \\
\hline
\end{tabular}

Article 2.a.(ii) Entry

\begin{tabular}{|c|l|c|}
\hline \multicolumn{1}{|c|}{ Condition } & \multicolumn{1}{c|}{ Message } & Type \\
\hline Agreed Information is blank. & Agreed Information is missing. & Info \\
\hline
\end{tabular}




\section{Article 2.a.(iii) Entry}

In addition to these rules, the rules for the composite Unit of Area and GeoCoordinates data types will be applied.

\begin{tabular}{|l|l|l|}
\hline \multicolumn{1}{|c|}{ Condition } & \multicolumn{1}{c|}{ Message } & \multicolumn{1}{c|}{ Type } \\
\hline Site Name is blank. & Site Name is missing. & Error \\
\hline Facility/LOF Identifiers is blank. & Facility/LOF Identifiers is missing. & Warning \\
\hline Building is blank & Building name is missing. & Error \\
\hline Building ID is blank. & Building ID is missing. & Warning \\
\hline Number of Floors is blank. & The Number of Floors in the building is missing. & Info \\
\hline Size is blank. & The Size of the building is missing. & Info \\
\hline $\begin{array}{l}\text { Size Units is blank and Size is not } \\
\text { blank. }\end{array}$ & $\begin{array}{l}\text { The Size of the building was entered, but Unit of area is } \\
\text { missing. }\end{array}$ & Error \\
\hline Use is blank. & The Use of the building is missing. & Error \\
\hline Previous Use is blank. & The Previous Use of the building is missing. & Info \\
\hline Contents is blank. & The Contents of the building is missing. & Warning \\
\hline Coordinates is blank. & Coordinates for the building is missing. & Warning \\
\hline $\begin{array}{l}\text { Attachments is blank and (if the } \\
\text { Entry is part of a Declaration) } \\
\text { Declaration attachments are also } \\
\text { blank. }\end{array}$ & No maps were listed or linked to the Declaration. & Warning \\
\hline
\end{tabular}

Article 2.a.(iv) Entry

In addition to these rules, the rules for the composite Location and Quantity data types will be applied (e.g., if a Quantity Value is entered but a Unit is not selected, the error from Quantity validation rules will be shown).

\begin{tabular}{|l|l|l|}
\hline \multicolumn{1}{|c|}{ Condition } & \multicolumn{1}{c|}{ Message } & \multicolumn{1}{c|}{ Type } \\
\hline Annex I Item is blank. & Annex I Item is missing. & Error \\
\hline Location is blank. & Locations are missing. & Error \\
\hline Brief Description is blank. & Brief Description is missing. & Info \\
\hline Capacity is blank. & Capacity is missing. & Error \\
\hline Extent Used is blank. & Extent Used is missing. & Info \\
\hline
\end{tabular}

\section{Article 2.a.(v) Mine/Plant Entry}

In addition to these rules, the rules for the composite Location and Quantity data types will be applied (e.g., if a Quantity Value is entered but a Unit is not selected, the error from Quantity validation rules will be shown).

\begin{tabular}{|l|l|l|}
\hline \multicolumn{1}{|c|}{ Condition } & \multicolumn{1}{c|}{ Message } & \multicolumn{1}{c|}{ Type } \\
\hline Mine/Plant Identifier is blank. & Mine/Plant Identifier is missing. & Error \\
\hline Location is blank. & Locations are missing. & Error \\
\hline Location Coordinates is blank. & Coordinates for the mine/concentration plan are missing. & Warning \\
\hline Operation is blank. & Operation is missing. & Error \\
\hline Estimated Annual Capacity is blank. & $\begin{array}{l}\text { Estimated Annual Capacity for this mine/concentration plant } \\
\text { is missing. }\end{array}$ & Error \\
\hline $\begin{array}{l}\text { Estimated Annual Capacity is non- } \\
\text { zero and Operation Status is “Closed } \\
\text { Down” or “Abandoned.” }\end{array}$ & $\begin{array}{l}\text { An Abandoned or Closed Down Mine or Plant should not } \\
\text { have an Estimated Annual Capacity greater than zero. }\end{array}$ & Warning \\
\hline $\begin{array}{l}\text { Actual Current Year Production is } \\
\text { blank and Mine/Plant Identifier } \\
\text { requires actual production to be } \\
\text { declared. }\end{array}$ & $\begin{array}{l}\text { Actual Current Year Production is missing, but has been } \\
\text { requested by the Agency. }\end{array}$ & Error \\
\hline
\end{tabular}




\begin{tabular}{|c|c|c|}
\hline Condition & Message & Type \\
\hline $\begin{array}{l}\text { Annual Current Year Production is } \\
\text { non-zero and Operation Status is } \\
\text { "Closed Down" or "Abandoned." }\end{array}$ & $\begin{array}{l}\text { An Abandoned or Closed Down Mine or Plant should not } \\
\text { have an Annual Current Year Production greater than zero. }\end{array}$ & Warning \\
\hline $\begin{array}{l}\text { Attachments is blank and (if the } \\
\text { Entry is part of a Declaration) } \\
\text { Declaration attachments are also } \\
\text { blank. }\end{array}$ & No maps were listed or linked to the Declaration. & Info \\
\hline
\end{tabular}

\section{Article 2.a.(v) State Total Entry}

In addition to these rules, the rules for the Quantity data type will be applied (e.g., if a Quantity Value is entered but a Unit is not selected, the error from Quantity validation rules will be shown).

\begin{tabular}{|l|l|l|}
\hline \multicolumn{1}{|c|}{ Condition } & \multicolumn{1}{c|}{ Message } & \multicolumn{1}{c|}{ Type } \\
\hline Operation is blank. & Operation is missing. & Error \\
\hline $\begin{array}{l}\text { Actual Current Year Production is } \\
\text { blank. }\end{array}$ & $\begin{array}{l}\text { Actual Current Year Production for the State as a whole is } \\
\text { missing. }\end{array}$ & Error \\
\hline
\end{tabular}

\section{Article 2.a.(vi).(a) Entry}

In addition to these rules, the rules for the Location and Quantity data types will be applied (e.g., if a Quantity Value is entered but a Unit is not selected, the error from Quantity will be shown).

\begin{tabular}{|l|l|l|}
\hline \multicolumn{1}{|c|}{ Condition } & \multicolumn{1}{c|}{ Message } & \multicolumn{1}{c|}{ Type } \\
\hline Locations is blank. & Locations are missing. & Error \\
\hline Chemical Composition is blank. & Chemical Composition is missing. & Error \\
\hline Quantity is blank. & Quantity is missing. & Error \\
\hline Intended Use Code is blank. & Intended Use Code is missing. & Error \\
\hline Intended Use is blank. & Intended Use is missing. & Error \\
\hline
\end{tabular}

\section{Article 2.a.(vi).(b) Entry}

In addition to these rules, the rules for the Quantity data type will be applied (e.g., if a Quantity Value is entered but a Unit is not selected, the error from Quantity validation rules will be shown).

\begin{tabular}{|l|l|l|}
\hline \multicolumn{1}{|c|}{ Condition } & \multicolumn{1}{c|}{ Message } & \multicolumn{1}{c|}{ Type } \\
\hline Destination is blank. & Destination is missing. & Error \\
\hline Interim Destination is blank. & Interim Destination is missing. & Info \\
\hline Chemical Composition is blank. & Chemical Composition is missing. & Error \\
\hline Quantity is blank. & Quantity is missing. & Error \\
\hline Intended Use Code is blank. & Intended Use Code is missing. & Error \\
\hline Intended Use is blank. & Intended Use is missing. & Error \\
\hline Export Date is blank. & Export Date is missing. & Error \\
\hline $\begin{array}{l}\text { Export Date is not within the Entry's } \\
\text { Reporting Date(s). }\end{array}$ & $\begin{array}{l}\text { The Export Date does not fall within this Entry's Reporting } \\
\text { Period. }\end{array}$ & Warning \\
\hline
\end{tabular}

\section{Article 2.a.(vi).(c) Entry}

In addition to these rules, the rules for the Location and Quantity data types will be applied (e.g., if a Quantity Value is entered but a Unit is not selected, the error from Quantity validation rules will be shown).

\begin{tabular}{|l|l|l|}
\hline \multicolumn{1}{|c|}{ Condition } & \multicolumn{1}{c|}{ Message } & \multicolumn{1}{c|}{ Type } \\
\hline Locations is blank. & Locations are missing. & Error \\
\hline Chemical Composition is blank. & Chemical Composition is missing. & Error \\
\hline Quantity is blank. & Quantity is missing. & Error \\
\hline Intended Use Code is blank. & Intended Use Code is missing. & Error \\
\hline Import Date is blank. & Import Date is missing. & Error \\
\hline
\end{tabular}




\begin{tabular}{|l|l|l|}
\hline \multicolumn{1}{|c|}{ Condition } & \multicolumn{1}{c|}{ Message } & \multicolumn{1}{c|}{ Type } \\
\hline $\begin{array}{l}\text { Import Date is not within the Entry's } \\
\text { Reporting Date(s). }\end{array}$ & $\begin{array}{l}\text { The Import Date does not fall within this Entry's Reporting } \\
\text { Period. }\end{array}$ & Warning \\
\hline Exporting State is blank. & Exporting State is missing. & Error \\
\hline
\end{tabular}

\section{Article 2.a.(vii) Entry}

\begin{tabular}{|l|l|l|}
\hline \multicolumn{1}{|c|}{ Condition } & \multicolumn{1}{c|}{ Message } & \multicolumn{1}{c|}{ Type } \\
\hline Locations is blank. & Locations are missing. & Error \\
\hline Exemption is blank. & Exemption are missing. & Error \\
\hline Material is blank. & Material Code is missing. & Error \\
\hline $\begin{array}{l}\text { Percent Uranium is blank and } \\
\text { Element/Material Code is Uranium. }\end{array}$ & $\begin{array}{l}\text { Percent Uranium is missing, but is required for materials that } \\
\text { contain Uranium }\end{array}$ & Error \\
\hline Weight is blank. & Weight is missing. & Error \\
\hline Intended Use Code is blank. & Intended Use Code is missing. & Error \\
\hline Intended Use is blank. & Intended Use is missing. & Error \\
\hline
\end{tabular}

\section{Article 2.a.(viii) Location Change Entry}

In addition to these rules, the rules for the Location and Quantity data types will be applied (e.g., if a Quantity Value is entered but a Unit is not selected, the error from Quantity validation rules will be shown).

\begin{tabular}{|l|l|l|}
\hline \multicolumn{1}{|c|}{ Condition } & \multicolumn{1}{c|}{ Message } & \multicolumn{1}{c|}{ Type } \\
\hline Waste Type is blank. & Waste Type is missing. & Error \\
\hline Number of Items is blank. & Number of Items is missing. & Error \\
\hline Type of Items is blank. & Type of Items is missing. & Error \\
\hline Conditioned Form is blank. & Conditioned Form is missing. & Info \\
\hline Quantity Pu is blank. & Quantity Pu is missing. & Info \\
\hline Quantity HEU is blank. & Quantity HEU is missing. & Info \\
\hline Quantity U-233 is blank & Quantity U-233 is missing. & Info \\
\hline Quantity Np/Am is blank. & Quantity Np/Am is missing. & Info \\
\hline All Quantity fields are blank. & $\begin{array}{l}\text { All Quantity fields are missing. Please fill in at least one of } \\
\text { the Quantity fields. }\end{array}$ & Error \\
\hline Previous Location & Previous Location is missing. & Error \\
\hline New Location & New Location is missing. & Error \\
\hline
\end{tabular}

\section{Article 2.a.(viii) Processing Entry}

In addition to these rules, the rules for the Quantity data type will be applied (e.g., if a Quantity Value is entered but a Unit is not selected, the error from Quantity validation rules will be shown).

\begin{tabular}{|l|l|l|}
\hline \multicolumn{1}{|c|}{ Condition } & \multicolumn{1}{c|}{ Message } & \multicolumn{1}{c|}{ Type } \\
\hline Waste Type is blank. & Waste Type is missing. & Error \\
\hline Number of Items is blank. & Number of Items is missing. & Error \\
\hline Type of Items is blank. & Type of Items is missing. & Error \\
\hline Conditioned Form is blank. & Conditioned Form is missing. & Info \\
\hline Quantity Pu is blank. & Quantity Pu is missing. & Info \\
\hline Quantity HEU is blank. & Quantity HEU is missing. & Info \\
\hline Quantity U-233 is blank & Quantity U-233 is missing. & Info \\
\hline Quantity Np/Am is blank. & Quantity Np/Am is missing. & Info \\
\hline All Quantity fields are blank. & $\begin{array}{l}\text { All Quantity fields are missing. Please fill in at least one of } \\
\text { the Quantity fields. }\end{array}$ & Error \\
\hline Locations is blank. & Locations are missing. & Error \\
\hline Processing Location is blank. & Processing Location is missing. & Error \\
\hline Start Date is blank. & Start Date is missing. & Info \\
\hline
\end{tabular}




\begin{tabular}{|l|l|l|}
\hline \multicolumn{1}{|c|}{ Condition } & \multicolumn{1}{c|}{ Message } & \multicolumn{1}{c|}{ Type } \\
\hline End Date is blank. & End Date is missing. & Info \\
\hline Start Date is greater than End Date. & $\begin{array}{l}\text { The Start Date comes after the End Date. The dates may be } \\
\text { reversed. }\end{array}$ & Warning \\
\hline $\begin{array}{l}\text { Start Date is greater than the end of } \\
\text { the Reporting Period. }\end{array}$ & $\begin{array}{l}\text { The Start Date indicates that processing started after the } \\
\text { Entry's Reporting Period. }\end{array}$ & Warning \\
& $\begin{array}{l}\text { Either a date was entered incorrectly, or this Activity may not } \\
\text { be declarable in this Submission. }\end{array}$ & $\begin{array}{l}\text { The End Date indicates that processing ended before the } \\
\text { Entry's Reporting Period. }\end{array}$ \\
\hline $\begin{array}{l}\text { End Date is less than the start of the } \\
\text { Reporting Period. }\end{array}$ & $\begin{array}{l}\text { Either a date was entered incorrectly, or this Activity may not } \\
\text { be declarable in this Submission. }\end{array}$ & Warning \\
\hline
\end{tabular}

\section{Article 2.a.(ix).(a) Entry}

In addition to these rules, the rules for the Location and Quantity data types will be applied (e.g., if a Quantity Value is entered but a Unit is not selected, the error from Quantity validation rules will be shown).

\begin{tabular}{|c|c|c|}
\hline Condition & Message & Type \\
\hline Item Identity is blank. & Item Identity is missing. & Error \\
\hline Annex II Paragraph is blank. & Annex II Paragraph is missing. & Error \\
\hline Quantity is blank. & Quantity is missing. & Error \\
\hline Locations of Intended Use is blank. & Locations of Intended Use are missing. & Error \\
\hline Export Date is blank. & Export Date is missing. & Error \\
\hline $\begin{array}{l}\text { Export Date is less than Reporting } \\
\text { Period Start. }\end{array}$ & $\begin{array}{l}\text { The Export Date indicates that the export happened before } \\
\text { the Entry's Reporting Period. } \\
\text { Either a date was entered incorrectly, or this Activity may not } \\
\text { be declarable in this Submission. }\end{array}$ & Warning \\
\hline $\begin{array}{l}\text { Export Date is greater than } \\
\text { Reporting Period End. }\end{array}$ & $\begin{array}{l}\text { The Export Date indicates that the export happened after the } \\
\text { Entry's Reporting Period. } \\
\text { Either a date was entered incorrectly, or this Activity may not } \\
\text { be declarable in this Submission. }\end{array}$ & Warning \\
\hline
\end{tabular}

\section{Article 2.a.(ix).(b) Entry}

In addition to these rules, the rules for the Location and Quantity data types will be applied (e.g., if a Quantity Value is entered but a Unit is not selected, the error from Quantity validation rules will be shown). 


\begin{tabular}{|c|c|c|}
\hline Condition & Message & Type \\
\hline Item Identity is blank. & Item Identity is missing. & Error \\
\hline Annex II Paragraph is blank. & Annex II Paragraph is missing. & Error \\
\hline Agency Request Number is blank. & Agency Request Number is missing. & Error \\
\hline $\begin{array}{l}\text { Agency Request Number is not in } \\
\text { "MC-CCC-\#\#” or “MC-CCC-\#\#.\#” } \\
\text { format. }\end{array}$ & $\begin{array}{l}\text { Agency Request Number is not in a standard format: either } \\
\text { MC-AAA-\#\# or MC-AAA-\#\#.\# (where AAA is a three-letter } \\
\text { country code and \# is a digit). } \\
\text { Ensure the number entered matches the number on the } \\
\text { Agency request exactly before submitting this information. }\end{array}$ & Warning \\
\hline Agency Request Date is blank. & Agency Request Date is missing. & Info \\
\hline Quantity is blank. & Quantity is missing. & Error \\
\hline Locations of Intended Use is blank. & Locations of Intended Use are missing. & Error \\
\hline $\begin{array}{l}\text { Import Date is blank and Not } \\
\text { Received is not checked. }\end{array}$ & $\begin{array}{l}\text { Import Date is missing. Either enter a date, or indicate that } \\
\text { you have not received the import by checking Not Received. }\end{array}$ & Error \\
\hline $\begin{array}{l}\text { Import Date is less than Reporting } \\
\text { Period Start. }\end{array}$ & $\begin{array}{l}\text { The Import Date indicates that the import happened before } \\
\text { the Entry’s Reporting Period. } \\
\text { Either a date was entered incorrectly, or this Activity may not } \\
\text { be declarable in this Submission. }\end{array}$ & Warning \\
\hline $\begin{array}{l}\text { Import Date is greater than } \\
\text { Reporting Period End. }\end{array}$ & $\begin{array}{l}\text { The Import Date indicates that the import happened after the } \\
\text { Entry's Reporting Period. } \\
\text { Either a date was entered incorrectly, or this Activity may not } \\
\text { be declarable in this Submission. }\end{array}$ & Warning \\
\hline
\end{tabular}

\section{Article 2.a.(x) Entry}

In addition to these rules, the rules for the Foreign Collaboration and Organization Involvement data types will be applied.

\begin{tabular}{|l|l|l|}
\hline \multicolumn{1}{|c|}{ Condition } & \multicolumn{1}{c|}{ Message } & \multicolumn{1}{c|}{ Type } \\
\hline Fuel Cycle Stage is blank. & Fuel Cycle Stages are missing. & Error \\
\hline Development Plans is blank. & Development Plans are missing. & Info \\
\hline R\&D Plans is blank. & R\&D Plans are missing. & Info \\
\hline $\begin{array}{l}\text { Development Plans and R\&D Plans } \\
\text { are blank. }\end{array}$ & Plan details (either R\&D or Development) are missing. & Error \\
\hline Collaboration is blank. & Collaboration information is missing. & Info \\
\hline Organizations is blank. & Organizational involvement is missing. & Info \\
\hline
\end{tabular}

\section{Article 2.b.(i) Entry}

In addition to these rules, the rules for the Location and Foreign Collaboration data types will be applied.

\begin{tabular}{|l|l|l|}
\hline \multicolumn{1}{|c|}{ Condition } & \multicolumn{1}{c|}{ Message } & \multicolumn{1}{c|}{ Type } \\
\hline Fuel Cycle Stages is blank. & Fuel Cycle Stages are missing. & Error \\
\hline Locations is blank. & Locations are missing. & Error \\
\hline Project Title is blank. & Project Title is missing. & Info \\
\hline Project ID is blank. & Project ID is missing. & Info \\
\hline Description is blank. & Description is missing. & Error \\
\hline Organizations is blank. & Organizations are missing. & Info \\
\hline Objectives is blank. & Objectives are missing. & Info \\
\hline $\begin{array}{l}\text { Degree to which Objectives Met is } \\
\text { blank. }\end{array}$ & Degree to which Objectives Met is missing. & Info \\
\hline Intended Applications is blank. & Intended Applications are missing. & Info \\
\hline Foreign Collaboration is blank. & Foreign Collaborations are missing. & Info \\
\hline
\end{tabular}




\begin{tabular}{|l|l|l|}
\hline \multicolumn{1}{|c|}{ Condition } & \multicolumn{1}{c|}{ Message } & \multicolumn{1}{c|}{ Type } \\
\hline Project Start Date & Project Start Date is missing. & Info \\
\hline Project End Date & Project End Date is missing. & Info \\
\hline
\end{tabular}

\section{Article 2.b.(ii) Entry}

In addition to these rules, the rules for the Location and Organization Involvement data types will be applied.

\begin{tabular}{|l|l|l|}
\hline \multicolumn{1}{|c|}{ Condition } & \multicolumn{1}{c|}{ Message } & \multicolumn{1}{c|}{ Type } \\
\hline Agency Request Number is blank. & Agency Request Number is missing. & Error \\
\hline $\begin{array}{l}\text { Agency Request Number is not in } \\
\text { "MC-CCC-\#\#" or “MC-CCC-\#\#.\#” } \\
\text { format. }\end{array}$ & $\begin{array}{l}\text { Agency Request Number is not in a standard format: either } \\
\text { MC-AAA-\#\# or MC-AAA-\#\#.\# (where AAA is a three-letter } \\
\text { country code and \# is a digit). }\end{array}$ & Warning \\
& $\begin{array}{l}\text { Ensure the number entered matches the number on the } \\
\text { Agency request exactly before submitting this information. }\end{array}$ & Info \\
\hline Agency Request Date & Agency Request Date is missing. & Error \\
\hline Locations is blank. & Locations are missing. & Error \\
\hline Related Site Code is blank. & $\begin{array}{l}\text { This Entry must be associated with a particular Site, but } \\
\text { Related Site Code is missing. }\end{array}$ & Info \\
\hline Physical Features is blank. & Physical Features are missing. & Info \\
\hline Description of Activities is blank. & Description of Activities is missing. & Info \\
\hline Carried Out By is blank. & The organization(s) who carried out the activity are missing. \\
\hline
\end{tabular}

\section{Article 2.c Entry}

\begin{tabular}{|c|c|c|}
\hline Condition & Message & Type \\
\hline Agency Request Number is blank. & Agency Request Number is missing. & Error \\
\hline $\begin{array}{l}\text { Agency Request Number is not in } \\
\text { "MC-CCC-\#\#" or "MC-CCC-\#\#.\#" } \\
\text { format. }\end{array}$ & $\begin{array}{l}\text { Agency Request Number is not in a standard format: either } \\
\text { MC-AAA-\#\# or MC-AAA-\#\#.\# (where AAA is a three-letter } \\
\text { country code and \# is a digit). } \\
\text { Ensure the number entered matches the number on the } \\
\text { Agency request exactly before submitting this information. }\end{array}$ & Warning \\
\hline Agency Request Date is blank. & Agency Request Date is missing. & Info \\
\hline Agency Question is blank. & Agency Question is missing. & Info \\
\hline Response is blank. & Response is missing. & Info \\
\hline $\begin{array}{l}\text { Response and Documents are both } \\
\text { blank. }\end{array}$ & $\begin{array}{l}\text { There was no Response or attachments included in the Entry } \\
\text { that can be used to answer the Agency request. }\end{array}$ & Warning \\
\hline
\end{tabular}

\section{Note Entry}

\begin{tabular}{|c|l|c|}
\hline \multicolumn{1}{|c|}{ Condition } & \multicolumn{1}{c|}{ Message } & \multicolumn{1}{c|}{ Type } \\
\hline General Description is blank. & General Description is missing. & Info \\
\hline
\end{tabular}

\subsubsection{Submission Data Structures}

\section{Submission}

All Submissions, regardless of Submission Type, should be validated using the same basic rules. Several validation rules, however, must take the Submission Type into account and will need to involve the data template plugin.

\begin{tabular}{|l|l|l|}
\hline \multicolumn{1}{|c|}{ Condition } & \multicolumn{1}{c|}{ Type } \\
\hline Description is blank. & Description is missing. & Info \\
\hline State is blank. & State is missing. & Error \\
\hline Submission Date is blank. & Submission Date is missing. & Error \\
\hline Declarable Comments is blank. & Declarable Comments are missing. & Info \\
\hline No Declarations are contained in the & Submissions should contain at least one Declaration. & Error \\
\hline
\end{tabular}




\begin{tabular}{|c|c|c|}
\hline \multirow{2}{*}{$\begin{array}{l}\text { Condition } \\
\text { Submission. }\end{array}$} & Message & Type \\
\hline & & \\
\hline $\begin{array}{l}\text { The Submission does not contain at } \\
\text { least one Declaration for each } \\
\text { required article. }\end{array}$ & $\begin{array}{l}\text { A [Submission Type] Submission does not contain at least } \\
\text { one of the following Declarations: [missing Declaration } \\
\text { Types]. }\end{array}$ & Error \\
\hline $\begin{array}{l}\text { The Submission contains more than } \\
\text { one Declaration for an AP article } \\
\text { other than Model AP 2.a.(iii) (or its } \\
\text { equivalent). }\end{array}$ & $\begin{array}{l}\text { The [Submission Type] should not contain more than one } \\
\text { Declaration for [AP Article]. }\end{array}$ & Warning \\
\hline $\begin{array}{l}\text { There is a gap between this } \\
\text { Declaration's starting number and } \\
\text { the highest starting number for a } \\
\text { Declaration with the same State and } \\
\text { Data Template Code. }\end{array}$ & $\begin{array}{l}\text { This Submission's starting number is [number]. Based on } \\
\text { other Submissions in your database, the starting number } \\
\text { should be [number]. }\end{array}$ & Warning \\
\hline $\begin{array}{l}\text { This Submission contains a } \\
\text { Declaration Number that matches } \\
\text { another Submission in the database } \\
\text { with the same State and Data } \\
\text { Template Code. }\end{array}$ & $\begin{array}{l}\text { This Submission contains Declaration(s) numbered } \\
\text { [numbers], which overlap with the numbering for another } \\
\text { Submission in your database. }\end{array}$ & Error \\
\hline
\end{tabular}

For the Model AP data template, Submission and Declaration articles match up as follows:

\begin{tabular}{|l|l|l|}
\hline Submission Type & Allowed AP Articles & Required AP Articles \\
\hline 3.a & 2.a.(i), (iii), (iv), (v), (vi)(a), (vii), (x), 2.b.(i), Note & $\begin{array}{l}\text { 2.a.(i), (iii), (iv), (v), (vi)(a), } \\
\text { (vii), (x), 2.b.(i) }\end{array}$ \\
\hline 3.b & 2.a.(i), (iii), (iv), (v), (vi)(a), (vii), (x), 2.b.(i), Note & $\begin{array}{l}\text { 2.a.(i), (iii), (iv), (v), (vi)(a), } \\
\text { (vii), (x), 2.b.(i) }\end{array}$ \\
\hline 3.c & 2.a.(vi)(b), (vi)(c), Note & 2.a.(vi)(b), (vi)(c), \\
\hline $3 . d$ & 2.a.(ix)(a), Note & 2.a.(ix)(a) \\
\hline 3.e & 2.a.(viii), Note & 2.a.(viii) \\
\hline 3.f & 2.a.(ii), Note & 2.a.(ii) \\
\hline $3 . g$ & 2.a.(ix)(b), Note & 2.a.(ix)(b) \\
\hline Response & 2.b.(ii), 2.c, Note & 2.b.(ii) or 2.c \\
\hline General & $\begin{array}{l}\text { 2.a.(i), (ii), (iii), (iv), (v), (vi), (vii), (viii), (ix), (x), 2.b.(i), } \\
\text { (ii), 2.c, Note }\end{array}$ & \\
\hline
\end{tabular}

\section{Declaration}

In addition to these rules, the rules for the Document data type will be applied.

\begin{tabular}{|c|c|c|}
\hline Condition & Message & Type \\
\hline Reporting Period End is blank. & Reporting Period End or As-Of Date is missing. & Error \\
\hline $\begin{array}{l}\text { Reporting Period Start is blank, and } \\
\text { the containing Submission is not an } \\
\text { Initial Submission type. }\end{array}$ & Reporting Period Start Date is missing. & Error \\
\hline Declarable Comments is blank. & Declarable Comments are missing. & Info \\
\hline $\begin{array}{l}\text { A New Information Declaration } \\
\text { contains no Entries. }\end{array}$ & $\begin{array}{l}\text { New Information Declarations should contain at least one } \\
\text { Entry. } \\
\text { If there should be no Entries in this Declaration, then it } \\
\text { should be marked Nothing to Declare. }\end{array}$ & Error \\
\hline $\begin{array}{l}\text { A Nothing to Declare Declaration } \\
\text { contains Entries. }\end{array}$ & $\begin{array}{l}\text { Nothing to Declare Declarations should contain no Entries. } \\
\text { If there should be Entries in this Declaration, then it should } \\
\text { be marked New Information or No Change. }\end{array}$ & Error \\
\hline
\end{tabular}




\begin{tabular}{|c|c|c|}
\hline Condition & Message & Type \\
\hline $\begin{array}{l}\text { A No Change Declaration contains } \\
\text { no Entries. }\end{array}$ & $\begin{array}{l}\text { No Change Declarations should contain at least one Entry. } \\
\text { If there should be no Entries in this Declaration, then it } \\
\text { should be marked Nothing to Declare. }\end{array}$ & Error \\
\hline $\begin{array}{l}\text { A No Change Declaration contains } \\
\text { Entries that are not No Change } \\
\text { Entries. }\end{array}$ & $\begin{array}{l}\text { No Change Declarations should contain only No Change } \\
\text { Entries. } \\
\text { If there should be a New Information or Update Entries in } \\
\text { this Declaration, then it should be marked New Information. }\end{array}$ & Error \\
\hline $\begin{array}{l}\text { A 2.a.(v) Mine/Plant Entry exists for } \\
\text { a Th or U operation, but the } \\
\text { Declaration does not contain a } \\
\text { 2.a.(v) State Total for a Th or U } \\
\text { operation. }\end{array}$ & $\begin{array}{l}\text { A 2.a.(v) Mine/Plant was declared for [operation], but there } \\
\text { is no corresponding 2.a.(v) State Total for [operation]. }\end{array}$ & Warning \\
\hline
\end{tabular}

\section{Declaration 2.a.(iii) Details}

\begin{tabular}{|c|c|c|}
\hline Condition & Message & Type \\
\hline Site Code is blank. & Site Code is missing. & Warning \\
\hline Site Name is blank. & Site Name is missing. & Error \\
\hline Working Hours is blank. & Working Hours is missing. & Info \\
\hline Site Holidays is blank. & Site Holidays is missing. & Info \\
\hline $\begin{array}{l}\text { Declaration contains two Entries } \\
\text { with the same Building ID. }\end{array}$ & $\begin{array}{l}\text { Two Entries have the same Building ID, which may indicate } \\
\text { an incorrect or duplicate Entry. } \\
\text { This warning may be ignored if this is intentional (for } \\
\text { example, if there are two Entries for different entities within } \\
\text { the same building.) }\end{array}$ & Warning \\
\hline
\end{tabular}

Declaration 2.a.(ix).(b) Details

\begin{tabular}{|l|l|l|}
\hline \multicolumn{1}{|c|}{ Condition } & \multicolumn{1}{c|}{ Message } & \multicolumn{1}{c|}{ Type } \\
\hline Agency Request Number is blank. & Agency Request Number is missing. & Error \\
\hline $\begin{array}{l}\text { Agency Request Number is not in } \\
\text { "MC-CCC-\#\#" or “MC-CCC-\#\#.\#” } \\
\text { format. }\end{array}$ & $\begin{array}{l}\text { Agency Request Number is not in a standard format: either } \\
\text { MC-AAA-\#\# or MC-AAA-\#\#.\# (where AAA is a three-letter } \\
\text { country code and \# is a digit). }\end{array}$ & \\
& $\begin{array}{l}\text { Warning } \\
\text { Ensure the number entered matches the number on the } \\
\text { Agency request exactly before submitting this information. }\end{array}$ & \\
\hline Agency Request Date is blank. & Agency Request Date is missing. & Info \\
\hline
\end{tabular}

\section{Declaration 2.b.(ii) Details}

\begin{tabular}{|l|l|l|}
\hline \multicolumn{1}{|c|}{ Condition } & \multicolumn{1}{c|}{ Message } & \multicolumn{1}{c|}{ Type } \\
\hline Agency Request Number is blank. & Agency Request Number is missing. & Error \\
\hline $\begin{array}{l}\text { Agency Request Number is not in } \\
\text { "MC-CC-\#\#” or “MC-CCC-\#\#.\#” } \\
\text { format. }\end{array}$ & $\begin{array}{l}\text { Agency Request Number is not in a standard format: either } \\
\text { MC-AAA-\#\# or MC-AAA-\#\#.\# (where AAA is a three-letter } \\
\text { country code and \# is a digit). }\end{array}$ & Warning \\
& $\begin{array}{l}\text { Ensure the number entered matches the number on the } \\
\text { Agency request exactly before submitting this information. }\end{array}$ & \\
\hline Agency Request Date is blank. & Agency Request Date is missing. & Info \\
\hline
\end{tabular}


Declaration 2.c Details

\begin{tabular}{|l|l|l|}
\hline \multicolumn{1}{|c|}{ Condition } & \multicolumn{1}{c|}{ Message } & \multicolumn{1}{c|}{ Type } \\
\hline Agency Request Number is blank. & Agency Request Number is missing. & Error \\
\hline $\begin{array}{l}\text { Agency Request Number is not in } \\
\text { "MC-CCC-\#\#” or “MC-CCC-\#\#.\#” } \\
\text { format. }\end{array}$ & $\begin{array}{l}\text { Agency Request Number is not in a standard format: either } \\
\text { MC-AAA-\#\# or MC-AAA-\#\#.\# (where AAA is a three-letter } \\
\text { country code and \# is a digit). }\end{array}$ & \\
& $\begin{array}{l}\text { Warning } \\
\text { Ensure the number entered matches the number on the } \\
\text { Agency request exactly before submitting this information. }\end{array}$ & \\
\hline Agency Request Date is blank. & Agency Request Date is missing. & Info \\
\hline
\end{tabular}

\subsubsection{Composite Data Types}

These data types may be used in multiple Entry, Declaration, and Submission data objects. Because of this, they will always be validated in the context of the declaration data objects that contain them.

An error in a composite data type may still prevent the export of a Submission. In this case, the user has two choices for how to resolve the situation: resolve the error(s) or (if possible, depending on the article type) leave the data type blank in the Entry, Declaration, or Submission.

If a user does not fill in any fields of a composite data type, it will be considered "blank" for the purpose of validation. The data type's validation rules will not apply, but those of the containing Entry, Declaration, or Submission will.

\section{Document}

\begin{tabular}{|l|l|l|}
\hline \multicolumn{1}{|c|}{ Condition } & \multicolumn{1}{c|}{ Message } & \multicolumn{1}{c|}{ Type } \\
\hline Document Title & Title is missing for this Document. & Error \\
\hline $\begin{array}{l}\text { Mime Type is blank and File } \\
\begin{array}{l}\text { Contents is not blank, or Mime Type } \\
\text { is not blank and File Contents is } \\
\text { blank. }\end{array}\end{array}$ & $\begin{array}{l}\text { The attachment for this Document was not recognized. Try } \\
\text { attaching the file again. }\end{array}$ & Error \\
\hline Description is blank. & & \\
\hline Document Type is blank. & Description is missing for this Document. & Info \\
\hline
\end{tabular}

\section{Facility/LOF Identifier}

\begin{tabular}{|c|c|c|}
\hline Condition & Message & Type \\
\hline $\begin{array}{l}\text { Facility Code and LOF Code are } \\
\text { both blank. }\end{array}$ & $\begin{array}{l}\text { Either a Facility Code or a LOF Code should be specified for } \\
\text { this identifier. Fill in a code or delete this identifier. }\end{array}$ & Error \\
\hline $\begin{array}{l}\text { Facility Code is not blank and not } \\
\text { found in Facilities lookup table. }\end{array}$ & $\begin{array}{l}\text { Facility Code “[code]” is not recognized. } \\
\text { This may be expected if the code was entered manually } \\
\text { rather than selected from a list. }\end{array}$ & Warning \\
\hline $\begin{array}{l}\text { LOF Code is not blank and not found } \\
\text { in the LOF's lookup table. }\end{array}$ & $\begin{array}{l}\text { LOF Code “[code]" is not recognized. } \\
\text { This may be expected if the code was entered manually } \\
\text { rather than selected from a list. }\end{array}$ & Warning \\
\hline $\begin{array}{l}\text { MBA Code is not blank and not } \\
\text { found in the LOF's lookup table. }\end{array}$ & $\begin{array}{l}\text { MBA Code “[code]” is not recognized. } \\
\text { This may be expected if the code was entered manually } \\
\text { rather than selected from a list. }\end{array}$ & Warning \\
\hline $\begin{array}{l}\text { KMP (Key Measurement Point) } \\
\text { Code is not blank and not found in } \\
\text { the LOF's lookup table. }\end{array}$ & $\begin{array}{l}\text { KMP Code “[code]” is not recognized. } \\
\text { This may be expected if the code was entered manually } \\
\text { rather than selected from a list. }\end{array}$ & Warning \\
\hline
\end{tabular}




\begin{tabular}{|c|c|c|}
\hline Condition & Message & Type \\
\hline $\begin{array}{l}\text { LOC ID is not blank and not found } \\
\text { in the LOF's lookup table. }\end{array}$ & $\begin{array}{l}\text { LOC ID “[id]" is not recognized. } \\
\text { This may be expected if the ID was entered manually rather } \\
\text { than selected from a list. }\end{array}$ & Warning \\
\hline $\begin{array}{l}\text { Facility Code is provided and one or } \\
\text { more LOF Identifier fields (LOF } \\
\text { Code, MBA Code, KMP Code, LOC } \\
\text { ID) are also provided }\end{array}$ & $\begin{array}{l}\text { [LOF Code/MBA Code/KMP Code/LOC ID] should not be } \\
\text { provided when Facility Code is provided. } \\
\text { If this Entry is associated with both Facility(s) and LOF(s), } \\
\text { create separate Facility/LOF Identifier records for each of } \\
\text { them. }\end{array}$ & Error \\
\hline $\begin{array}{l}\text { Facility Code, LOF Code, MBA } \\
\text { Code, KMP Code, and LOC ID are } \\
\text { blank. }\end{array}$ & Facility or LOF identification information is missing. & Warning \\
\hline $\begin{array}{l}\text { MBA Code is not provided, but } \\
\text { KMP Code or LOC ID is provided. }\end{array}$ & MBA Code cannot be blank if KMP Code is provided. & Error \\
\hline $\begin{array}{l}\text { MBA Code and/or KMP Code is not } \\
\text { provided, but LOC ID is provided. }\end{array}$ & $\begin{array}{l}\text { LOC ID cannot be provided if MBA Code and KMP Code } \\
\text { are not both provided. }\end{array}$ & Error \\
\hline
\end{tabular}

\section{Foreign Collaboration}

\begin{tabular}{|l|l|l|}
\hline \multicolumn{1}{|c|}{ Condition } & \multicolumn{1}{c|}{ Message } & \multicolumn{1}{c|}{ Type } \\
\hline State is blank. & State is missing for this Foreign Collaboration. & Error \\
\hline Organization is blank. & Organization name is missing for this Foreign Collaboration. & Error \\
\hline Address is blank. & $\begin{array}{l}\text { The Address of the organization is missing for this Foreign } \\
\text { Collaboration. }\end{array}$ & Error \\
\hline Involvement is blank. & $\begin{array}{l}\text { A description of the Involvement of the organization is } \\
\text { missing for this Foreign Collaboration. }\end{array}$ & Warning \\
\hline
\end{tabular}

\section{GeoCoordinates}

\begin{tabular}{|l|l|l|}
\hline \multicolumn{1}{|c|}{ Condition } & \multicolumn{1}{c|}{ Message } & \multicolumn{1}{c|}{ Type } \\
\hline $\begin{array}{l}\text { Latitude is blank or incomplete, and } \\
\text { Longitude is complete. }\end{array}$ & Latitude is missing for this GeoCoordinate. & Error \\
\hline $\begin{array}{l}\text { Longitude is blank or incomplete, } \\
\text { and Latitude is complete. }\end{array}$ & Longitude is missing for this GeoCoordinate. & Error \\
\hline
\end{tabular}

\section{Location}

In addition to these rules, the rules for the GeoCoordinates data type will be applied.

\begin{tabular}{|l|l|l|}
\hline \multicolumn{1}{|c|}{ Condition } & \multicolumn{1}{c|}{ Message } & \multicolumn{1}{c|}{ Type } \\
\hline Name is blank. & Name is missing for this Location. & Error \\
\hline Address is blank. & Address is missing for this Location. & Error \\
\hline Building is blank. & Building is missing for this Location. & Warning \\
\hline Room is blank. & Room is missing for this Location. & Info \\
\hline Subarea is blank. & Subarea is missing for this Location. & Wnfo \\
\hline Facility/LOF is blank. & Facility/LOF is missing for this Location. & Warning \\
\hline $\begin{array}{l}\text { Facility/LOF was entered, but is not } \\
\text { found in the Facility/LOF lookup. }\end{array}$ & $\begin{array}{l}\text { The Facility/LOF entered in this Location is not found in the } \\
\text { Facility/LOF lookup list. } \\
\text { If this Location was created by another user, it may be } \\
\text { correct. Otherwise, you may need to check the value in this } \\
\text { field. }\end{array}$ & \\
\hline
\end{tabular}


Organization Involvement

\begin{tabular}{|l|l|l|}
\hline \multicolumn{1}{|c|}{ Condition } & \multicolumn{1}{c|}{ Message } & \multicolumn{1}{c|}{ Type } \\
\hline Organization is blank. & $\begin{array}{l}\text { Organization is missing for this Organization Involvement } \\
\text { record. }\end{array}$ & Error \\
\hline Brief Description is blank. & $\begin{array}{l}\text { Brief Description is missing for this Organization } \\
\text { Involvement record. }\end{array}$ & Error \\
\hline
\end{tabular}

Quantity
\begin{tabular}{|l|l|l|}
\hline \multicolumn{1}{|c|}{ Condition } & \multicolumn{1}{c|}{ Message } & Type \\
\hline Value is blank, but Units is not. & Value is missing for this Quantity. & Error \\
\hline Units is blank, but Value is not. & Unit of measurement is missing for this Quantity. & Error \\
\hline
\end{tabular}

\section{Reference}

\begin{tabular}{|c|c|c|}
\hline Condition & Message & Type \\
\hline Reference Type is blank. & Reference Type is missing for this Reference. & Error \\
\hline $\begin{array}{l}\text { State is blank and Internal ID is } \\
\text { blank. }\end{array}$ & State is missing for this Reference. & Info \\
\hline $\begin{array}{l}\text { Declaration is blank and Internal ID } \\
\text { is not set. }\end{array}$ & Declaration Number is missing for this Reference. & Error \\
\hline $\begin{array}{l}\text { Entry is blank and Internal ID is not } \\
\text { set. }\end{array}$ & Entry Number is missing for this Reference. & Warning \\
\hline Notes is blank. & Notes are missing for this Reference. & Info \\
\hline $\begin{array}{l}\text { The Entry is contained by a } \\
\text { Declaration. Declaration is blank. } \\
\text { The Entry number is not found in } \\
\text { this Declaration. }\end{array}$ & $\begin{array}{l}\text { The Entry Number for this Reference was not found in the } \\
\text { current Declaration. } \\
\text { You may need to add a Declaration Number, or the Entry } \\
\text { Number may have been entered incorrectly. }\end{array}$ & Error \\
\hline $\begin{array}{l}\text { The Internal ID was not found in the } \\
\text { index of Internal IDs in this instance } \\
\text { of PR3. }\end{array}$ & $\begin{array}{l}\text { This Reference could not be validated. } \\
\text { If this Reference was created by another user, it may be } \\
\text { correct. Otherwise, you may need to select a new Entry for } \\
\text { this Reference or enter an explicit number. }\end{array}$ & Warning \\
\hline $\begin{array}{l}\text { The combination of State, } \\
\text { Declaration, and Entry was not found } \\
\text { in the index of Entry references in } \\
\text { this instance of PR3. }\end{array}$ & $\begin{array}{l}\text { The Entry Number in this Reference could not be validated. } \\
\text { If this Reference was created by another user, it may be } \\
\text { correct. Otherwise, you may need to check the explicit } \\
\text { number that was entered or select an Entry. }\end{array}$ & Warning \\
\hline
\end{tabular}

\subsubsection{AP for China}

\section{Article 2.a.(i) Entry}

Validation rules will be the same as for Model AP 2.a.(iv).

\section{Article 2.a.(ii) Mine/Plant Entry}

Validation rules will be the same as for Model AP 2.a.(v) Mine/Plant.

\section{Article 2.a.(ii) State Total Entry}

Validation rules will be the same as for Model AP 2.a.(v) State Total.

\section{Article 2.a.(iii).(a) Entry}

Validation rules will be the same as for Model AP 2.a.(vi).(b).

Article 2.a.(iii).(b) Entry

Validation rules will be the same as for Model AP 2.a.(iv).(c). 


\section{Article 2.a.(iv) Entry}

Validation rules will be the same as for Model AP 2.a.(vii).

Article 2.a.(v) Export Entry

Validation rules will be the same as for Model AP 2.a.(viii) Import, plus the following additional rule:

\begin{tabular}{|l|l|l|}
\hline \multicolumn{1}{|c|}{ Condition } & \multicolumn{1}{c|}{ Message } & \multicolumn{1}{c|}{ Type } \\
\hline Export Date is blank. & Export Date is missing. & Error \\
\hline $\begin{array}{l}\text { Export Date is not within the Entry's } \\
\text { Reporting Date(s). }\end{array}$ & $\begin{array}{l}\text { The Export Date does not fall within this Entry's Reporting } \\
\text { Period. }\end{array}$ & Warning \\
\hline
\end{tabular}

\section{Article 2.a.(v) Import Entry}

Validation rules will be the same as for Model AP 2.a.(viii) Export, plus the following additional rule:

\begin{tabular}{|l|l|l|}
\hline \multicolumn{1}{|c|}{ Condition } & \multicolumn{1}{c|}{ Message } & \multicolumn{1}{c|}{ Type } \\
\hline Import Date is blank. & Import Date is missing. & Error \\
\hline $\begin{array}{l}\text { Import Date is not within the Entry's } \\
\text { Reporting Date(s). }\end{array}$ & $\begin{array}{l}\text { The Import Date does not fall within this Entry’s Reporting } \\
\text { Period. }\end{array}$ & Warning \\
\hline
\end{tabular}

\section{Article 2.a.(vi) Entry}

Validation rules will be the same as for Model AP 2.a.(ix).(a).

\section{Article 2.a.(vii) Entry}

Validation rules will be the same as for Model AP 2.a.(x).

\section{Article 2.b Entry}

Validation rules will be the same as for Model AP 2.a.(i), plus the following additional rules:

\begin{tabular}{|l|l|l|}
\hline \multicolumn{1}{|c|}{ Condition } & \multicolumn{1}{c|}{ Message } & \multicolumn{1}{c|}{ Type } \\
\hline Agency Request Number is blank. & Agency Request Number is missing. & Warning \\
\hline $\begin{array}{l}\text { Agency Request Number is not in } \\
\text { "MC-CCC-\#\#” or “MC-CCC-\#\#.\#” } \\
\text { format. }\end{array}$ & $\begin{array}{l}\text { Agency Request Number is not in a standard format: either } \\
\text { MC-AAA-\#\# or MC-AAA-\#\#.\# (where AAA is a three-letter } \\
\text { country code and \# is a digit). }\end{array}$ & Warning \\
& $\begin{array}{l}\text { Ensure the number entered matches the number on the } \\
\text { Agency request exactly before submitting this information. }\end{array}$ & Info \\
\hline Agency Request Date is blank. & Agency Request Date is missing. & \\
\hline
\end{tabular}

Article 2.c Entry

Validation rules will be the same as for Model AP 2.c.

\section{Article 2.d Entry}

Validation rules will be the same as for Model AP 2.c.

\section{Note Entry}

Validation rules will be the same as for Model AP Note.

\section{Submission}

Validation rules will be the same as for Model AP Submission.

\section{Declaration}

Validation rules will be the same as for Model AP Declaration. 


\section{Declaration Details 2.b}

Validation rules will be the same as for Model AP Declaration Details 2.b, plus the following additional rules:

\begin{tabular}{|c|c|c|}
\hline Condition & Message & Type \\
\hline Agency Request Number is blank. & Agency Request Number is missing. & Warning \\
\hline $\begin{array}{l}\text { Agency Request Number is not in } \\
\text { "MC-CCC-\#\#” or “MC-CCC-\#\#.\#” } \\
\text { format. }\end{array}$ & $\begin{array}{l}\text { Agency Request Number is not in a standard format: either } \\
\text { MC-AAA-\#\# or MC-AAA-\#\#.\# (where AAA is a three-letter } \\
\text { country code and \# is a digit). } \\
\text { Ensure the number entered matches the number on the } \\
\text { Agency request exactly before submitting this information. }\end{array}$ & Warning \\
\hline Agency Request Date is blank. & Agency Request Date is missing. & Info \\
\hline
\end{tabular}

\subsubsection{AP for France}

\section{Article 2.a.(i) Entry}

Validation rules will be the same as for Model AP 2.a.(i), plus the following additional rules:

\begin{tabular}{|l|l|l|}
\hline \multicolumn{1}{|c|}{ Condition } & \multicolumn{1}{c|}{ Message } & \multicolumn{1}{c|}{ Type } \\
\hline Locations of the activities is blank. & Locations of the activities are missing. & Info \\
\hline Access information is blank. & Access information is missing. & Info \\
\hline
\end{tabular}

\section{Article 2.a.(ii) Entry}

Validation rules will be the same as for Model AP 2.a.(ii).

\section{Article 2.a.(iii) Entry}

Validation rules will be the same as for Model AP 2.a.(iv), plus the following additional rules:

\begin{tabular}{|l|l|l|}
\hline \multicolumn{1}{|c|}{ Condition } & \multicolumn{1}{c|}{ Message } & \multicolumn{1}{c|}{ Type } \\
\hline Locations of the activities is blank. & Locations of the activities are missing. & Info \\
\hline $\begin{array}{l}\text { Information on the NNWS entity is } \\
\text { blank. }\end{array}$ & Information on the NNWS entity is missing. & Info \\
\hline Access information is blank. & Access information is missing. & Info \\
\hline
\end{tabular}

\section{Article 2.a.(iv) Mine/Plant Entry}

Validation rules will be the same as for Model AP 2.a.(v) Mine/Plant.

\section{Article 2.a.(iv) State Total Entry}

Validation rules will be the same as for Model AP 2.a.(v) State Total.

\section{Article 2.a.(v)(a) Entry}

Validation rules will be the same as for Model AP 2.a.(vi)(b).

\section{Article 2.a.(v)(b) Entry}

Validation rules will be the same as for Model AP 2.a.(vi)(c).

\section{Article 2.a.(vi) Export Entry}

Validation rules will be the same as for Model AP 2.a.(viii) Location Change, minus any rules that specifically reference the Waste Type or Quantity Np/Am fields. The following additional rules will be included: 


\begin{tabular}{|l|l|l|}
\hline \multicolumn{1}{|c|}{ Condition } & \multicolumn{1}{c|}{ Message } & \multicolumn{1}{c|}{ Type } \\
\hline Export Date is blank. & Export Date is missing. & Error \\
\hline $\begin{array}{l}\text { Export Date is not within the Entry's } \\
\text { Reporting Date(s). }\end{array}$ & $\begin{array}{l}\text { The Export Date does not fall within this Entry's Reporting } \\
\text { Period. }\end{array}$ & Warning \\
\hline
\end{tabular}

\section{Article 2.a.(vi) Import Entry}

Validation rules will be the same as for Model AP 2.a.(viii) Location Change, minus any rules that specifically reference the Waste Type or Quantity Np/Am fields. The following additional rules will be included:

\begin{tabular}{|l|l|l|}
\hline \multicolumn{1}{|c|}{ Condition } & \multicolumn{1}{c|}{ Message } & \multicolumn{1}{c|}{ Type } \\
\hline Import Date is blank. & Import Date is missing. & Error \\
\hline $\begin{array}{l}\text { Import Date is not within the Entry's } \\
\text { Reporting Date(s). }\end{array}$ & $\begin{array}{l}\text { The Import Date does not fall within this Entry's Reporting } \\
\text { Period. }\end{array}$ & Warning \\
\hline
\end{tabular}

\section{Article 2.a.(vii)(a) Entry}

Validation rules will be the same as for Model AP 2.a.(ix)(a), plus the following additional rules:

\begin{tabular}{|c|l|c|}
\hline \multicolumn{1}{|c|}{ Condition } & \multicolumn{1}{|c|}{ Message } & Type \\
\hline Annex I Paragraph is blank. & Annex I Paragraph is missing. & Error \\
\hline
\end{tabular}

\section{Article 2.a.(vii)(b) Entry}

Validation rules will be the same as for Model AP 2.a.(ix)(b), plus the following additional rules:

\begin{tabular}{|c|l|c|}
\hline \multicolumn{1}{|c|}{ Condition } & \multicolumn{1}{|c|}{ Message } & Type \\
\hline Annex I Paragraph is blank. & Annex I Paragraph is missing. & Error \\
\hline
\end{tabular}

\section{Article 2.a.(viii) Entry}

Validation rules will be the same as for Model AP 2.a.(x), minus any rules that specifically reference the Development Plans field. The following additional rules will be included:

\begin{tabular}{|l|l|c|}
\hline \multicolumn{1}{|c|}{ Condition } & \multicolumn{1}{c|}{ Message } & Type \\
\hline $\begin{array}{l}\text { Information on the NNWS entity is } \\
\text { blank. }\end{array}$ & Information on the NNWS entity is missing. & Info \\
\hline
\end{tabular}

\section{Article 2.b Entry}

Validation rules will be the same as for Model AP 2.b.(i).

\section{Article 2.c Entry}

Validation rules will be the same as for Model AP 2.c.

\section{Note Entry}

Validation rules will be the same as for Model AP Note.

\section{Submission}

Validation rules will be the same as for Model AP Submission.

\section{Declaration}

Validation rules will be the same as for Model AP Declaration.

\subsubsection{AP for India}

\section{Article 2.a.(i) Entry}

Validation rules will be the same as for Model AP 2.a.(vi)(b). 


\section{Article 2.a.(ii) Entry}

Validation rules will be the same as for Model AP 2.a.(ix)(a).

\section{Article 2.b Entry}

Validation rules will be the same as for Model AP 2.c.

\subsubsection{AP for Russia}

\section{Article 2.a.(i) Entry}

Validation rules will be the same as for Model AP 2.a.(i), plus the following additional rules:

\begin{tabular}{|l|l|l|}
\hline \multicolumn{1}{|c|}{ Condition } & \multicolumn{1}{c|}{ Message } & \multicolumn{1}{c|}{ Type } \\
\hline Locations of the activities is blank. & Locations of the activities were not entered. & Info \\
\hline Access information is blank. & Access information was not entered. & Info \\
\hline
\end{tabular}

\section{Article 2.a.(ii) Entry}

Validation rules will be the same as for Model AP 2.a.(ii).

\section{Article 2.a.(iii) Entry}

Validation rules will be the same as for Model AP 2.a.(iv), plus the following additional rules:

\begin{tabular}{|l|l|l|}
\hline \multicolumn{1}{|c|}{ Condition } & \multicolumn{1}{c|}{ Message } & \multicolumn{1}{c|}{ Type } \\
\hline Locations of the activities is blank. & Locations of the activities were not entered. & Warning \\
\hline $\begin{array}{l}\text { Information on the NNWS entity is } \\
\text { blank. }\end{array}$ & Information on the NNWS entity is blank. & Info \\
\hline Access information is blank. & Access information was not entered. & Info \\
\hline
\end{tabular}

Article 2.a.(iv) Mine/Plant Entry

Validation rules will be the same as for Model AP 2.a.(v) Mine/Plant.

Article 2.a.(iv) State Total Entry

Validation rules will be the same as for Model AP 2.a.(v) State Total.

\section{Article 2.a.(v)(a) Entry}

Validation rules will be the same as for Model AP 2.a.(vi)(b).

\section{Article 2.a.(v)(b) Entry}

Validation rules will be the same as for Model AP 2.a.(vi)(c).

\section{Article 2.a.(vi) Entry}

Validation rules will be the same as for Model AP 2.a.(viii) Location Change, minus any rules that specifically reference the Waste Type or Quantity Np/Am fields. The following additional rules will be included:

\begin{tabular}{|l|l|l|}
\hline \multicolumn{1}{|c|}{ Condition } & \multicolumn{1}{c|}{ Message } & \multicolumn{1}{c|}{ Type } \\
\hline Export Date is blank. & Export Date was not entered. & Error \\
\hline $\begin{array}{l}\text { Export Date is not within the Entry's } \\
\text { Reporting Date(s). }\end{array}$ & $\begin{array}{l}\text { The Export Date entered does not fall within this Entry's } \\
\text { Reporting Period. }\end{array}$ & Warning \\
\hline
\end{tabular}

\section{Article 2.a.(vii)(a) Entry}

Validation rules will be the same as for Model AP 2.a.(ix)(a). 


\section{Article 2.a.(vii)(b) Entry}

Validation rules will be the same as for Model AP 2.a.(ix)(b).

Article 2.b Entry

Validation rules will be the same as for Model AP 2.b.(i).

Article 2.c Entry

Validation rules will be the same as for Model AP 2.c.

\section{Note Entry}

Validation rules will be the same as for Model AP Note.

\section{Submission}

Validation rules will be the same as for Model AP Submission.

\section{Declaration}

Validation rules will be the same as for Model AP Declaration.

\subsubsection{AP for the UK}

Article 2.a.(i) Entry

Validation rules will be the same as for Model AP 2.a.(i).

Article 2.a.(ii) Entry

Validation rules will be the same as for Model AP 2.a.(ii).

\section{Article 2.a.(iii) Entry}

Validation rules will be the same as for Model AP 2.a.(iv).

\section{Article 2.a.(iv) Mine/Plant Entry}

Validation rules will be the same as for Model AP 2.a.(v) Mine/Plant.

\section{Article 2.a.(iv) State Total Entry}

Validation rules will be the same as for Model AP 2.a.(v) State Total.

\section{Article 2.a.(v)(a) Entry}

Validation rules will be the same as for Model AP 2.a.(vi)(b).

\section{Article 2.a.(v)(b) Entry}

Validation rules will be the same as for Model AP 2.a.(vi)(c).

Article 2.a.(vi) Entry

Validation rules will be the same as for Model AP 2.a.(vii).

\section{Article 2.a.(vii) Export Entry}

Validation rules will be the same as for Model AP 2.a.(viii) Location Change, minus any rules that specifically reference the Waste Type or Quantity Np/Am fields. The following additional rules will be included: 


\begin{tabular}{|l|l|l|}
\hline \multicolumn{1}{|c|}{ Condition } & \multicolumn{1}{c|}{ Message } & \multicolumn{1}{c|}{ Type } \\
\hline Export Date is blank. & Export Date is missing. & Error \\
\hline $\begin{array}{l}\text { Export Date is not within the Entry's } \\
\text { Reporting Date(s). }\end{array}$ & $\begin{array}{l}\text { The Export Date does not fall within this Entry's Reporting } \\
\text { Period. }\end{array}$ & Warning \\
\hline
\end{tabular}

\section{Article 2.a.(vii) Import Entry}

Validation rules will be the same as for Model AP 2.a.(viii) Location Change, minus any rules that specifically reference the Waste Type or Quantity Np/Am fields. The following additional rules will be included:

\begin{tabular}{|l|l|l|}
\hline \multicolumn{1}{|c|}{ Condition } & \multicolumn{1}{c|}{ Message } & \multicolumn{1}{c|}{ Type } \\
\hline Import Date is blank. & Import Date is missing. & Error \\
\hline $\begin{array}{l}\text { Import Date is not within the Entry's } \\
\text { Reporting Date(s). }\end{array}$ & $\begin{array}{l}\text { The Import Date does not fall within this Entry's Reporting } \\
\text { Period. }\end{array}$ & Warning \\
\hline
\end{tabular}

\section{Article 2.a.(viii)(a) Entry}

Validation rules will be the same as for Model AP 2.a.(ix)(a).

Article 2.a.(viii)(b) Entry

Validation rules will be the same as for Model AP 2.a.(ix)(b).

Article 2.a.(ix) Entry

Validation rules will be the same as for Model AP 2.a.(x).

\section{Article 2.b Entry}

Validation rules will be the same as for Model AP 2.b.(i).

\section{Article 2.c Entry}

Validation rules will be the same as for Model AP 2.c.

\section{Note Entry}

Validation rules will be the same as for Model AP Note.

\section{Submission}

Validation rules will be the same as for Model AP Submission.

\section{Declaration}

Validation rules will be the same as for Model AP Declaration. 
APPENDIX A. GLOSSARY 



\section{APPENDIX A. GLOSSARY}

Additional Protocol (AP)

Article 2

Article 3

Contributor

Declarable Data

Declaration

Entry

Entry Into Force (EIF) Date

Final Entry

Format Guidelines
Refers to the additional reporting agreements following the "Model Protocol Additional to the Agreement(s) between State(s) and the International Atomic Energy Agency for the Application of Safeguards" (INFCIRC/540) (Corrected) document.

That section of an Additional Protocol that defines the types of information that a State must provide to the IAEA.

That section of an Additional Protocol that defines the frequencies at which different types of information defined in Article 2 must be provided to the IAEA.

An organization or individual in a Member State that is responsible for preparing and submitting a portion of the State's declaration data to the SRA or to another Contributor or reviewer for eventual submission to the SRA.

Data in fields that are intended to be transmitted to the IAEA as part of the declaration process. When the PR3 prepares a Submission to be transferred to the IAEA, it will contain only Declarable data.

Information submitted to the IAEA by a State about its nuclear program and related activities pursuant to an AP agreement. A Declaration consists of header information and a collection of zero or more entries that apply to a specific paragraph of AP Article 2 in a format that meets the requirements of that article.

An Entry is a description of a specific activity and/or location for a specific reporting period that is reported to the IAEA pursuant to a specific paragraph of AP Article 2.

The date on which the Agency receives from a State written notification that the State's statutory and constitutional requirements for entry into force have been met. A State may, at any date before its AP enters into force, declare that it will apply the AP provisionally.

An Entry reporting an activity or location that is completed or closed for which future updates are not expected to be submitted to the IAEA.

An IAEA publication entitled Guidelines and Format for the Preparation and Submission of the Declarations Pursuant to Article 2 \& 3 of the Model Protocol Additional to Safeguards Agreements (May 2004). 
Internal Data

Legacy Data

No Change Entry

Nothing to Declare Declaration

State or Regional Authority (SRA)

Submission

Update Entry
Data in fields that are intended to be withheld from the IAEA and used only for internal State record-keeping or for tracking the declaration development process. When the PR3 prepares a Submission to be transferred to the IAEA, it will contain no Internal Data.

For the purposes of this document, legacy data refers to any Declaration data created in Protocol Reporter 1.0 or Protocol Reporter 2.0 format.

A special case of Update Entry indicating that the information previously submitted for an activity or location remains accurate for the indicated reporting period. In a Declaration, this indicates that the absence of new information related to that activity or location is intended.

A Declaration indicating that no activities or locations are declarable under the associated AP Article for the indicated reporting period. In a Submission, this indicates that the absence of Declaration data related to that AP Article is intended.

If a State has declarable activities or locations, but they have not changed since the last Submission, use a No Change Declaration instead.

The organization in a Member State responsible for preparing, certifying, and submitting declarations to the IAEA.

A set of one or more Declarations that are compiled by a State to be provided to the IAEA in accordance with AP Article 3.

An Entry that provides information related to a specific activity or location reported in a previously submitted Entry. The Update Entry shall include a reference to the previous Entry. 


\section{APPENDIX B. TARGET TOOLS}





\section{APPENDIX B. TARGET TOOLS}

\section{B.1 Microsoft .NET Framework 4.0 / Windows Presentation Foundation}

The visual prototype was developed using Windows Presentation Foundation (WPF) and .NET 4.0. If another platform or UI framework is used, the PR3 may look and/or behave differently than described.

.NET 4.0 was chosen as it includes all the WPF features necessary to implement the visual prototype.

\section{B.2 Telerik Controls for WPF}

The visual prototype was developed using Telerik Controls for WPF. This toolset was chosen primarily for its enhanced data grid control, which has built-in column filtering and grouping.

If another library is used to provide these features (or these features are developed from scratch), the PR3 may look and/or behave differently than described.

\section{B.3 Microsoft Report Viewer / Microsoft SQL Server Data Tools - Business Intelligence for Visual Studio}

The visual prototype used Microsoft Report Viewer for all report prototypes. It was chosen for its ability to generate printable reports and export to multiple file formats.

The Report Viewer redistributable is free and could be bundled into the PR3 installer. The report designer is included in the Microsoft SQL Server Data Tools - Business Intelligence for Visual Studio, which is provided as a free download from the Microsoft website.

If another reporting tool is used, the style and layout, printing capabilities, and export capabilities for PR3 reports may be different than described.

\section{B.4 RavenDB or NDatabase}

The Components section of the SDS was written with the intention of using a document or object database for data storage. This would allow complex data types to be stored directly rather than being translated into a table structure as in a relational database. It would also allow easier access to a user's entire collection of PR3 data than storage in individual files (such as XML).

If a database is used in the PR3, it must meet the following requirements:

- It must run embedded in the PR3 process. (That is, it must not require a separate Windows service or executable to be running.)

- $\quad$ It must not require any additional software installations.

- It must not require the user to purchase an additional license.

RavenDB and NDatabase were identified as possible candidates for databases because they meet all of these requirements. The choice may be dictated by licensing; RavenDB requires developers to purchase a commercial license; NDatabase is free under the GNU Lesser General Public License. 


\section{B.5 Lucene.NET}

The Search Component of the SDS was designed with the intention of using a separate library such as Lucene.NET for full-text searching. Lucene.NET runs embedded in the PR3 process and requires no additional software installation or user licensing.

While many document databases (see Sect. B.4) may include some form of full-text searching, it may be helpful to index PR3 data with Lucene.NET as well. This may give developers greater control over how data is indexed, allowing for more complex search functionality (for example, allowing the user to search for specific text in specific fields). 
APPENDIX C. SRS CROSS-REFERENCE 



\section{APPENDIX C. SRS CROSS-REFERENCE}

\section{Operational Environment (OE)}

\begin{tabular}{|c|c|c|}
\hline Reqmt-ID & Requirement & Design Elements \\
\hline OE-001 & $\begin{array}{l}\text { The system shall be able to operate on a } \\
\text { single Windows-based computer. }\end{array}$ & 1.4 \\
\hline OE-002 & $\begin{array}{l}\text { The system shall be capable of running on } \\
\text { either 32-bit or 64-bit computing } \\
\text { platforms. }\end{array}$ & 1.4 \\
\hline OE-003 & $\begin{array}{l}\text { The system shall be capable of running } \\
\text { under Microsoft Windows XP operating } \\
\text { system and later versions of Windows } \\
\text { through Windows 8.1. }\end{array}$ & APPENDIX B.1 \\
\hline OE-004 & $\begin{array}{l}\text { Installation and operation of the system } \\
\text { shall not require additional software or } \\
\text { special environments that lead to the need } \\
\text { for supplementary software licenses. }\end{array}$ & 1.4 \\
\hline OE-005 & $\begin{array}{l}\text { Human-readable source code for the } \\
\text { system shall be available to the State. }\end{array}$ & $\begin{array}{l}\text { N/A - This is a development process rather than a } \\
\text { design issue. }\end{array}$ \\
\hline
\end{tabular}

\section{System Configuration (SC)}

\begin{tabular}{|c|c|c|}
\hline Reqmt-ID & Requirement & Design Elements \\
\hline SC-001 & $\begin{array}{l}\text { The system and all associated } \\
\text { components (including help files) shall be } \\
\text { written in the English language. }\end{array}$ & N/A \\
\hline SC-002 & $\begin{array}{l}\text { At a minimum, the system shall support a } \\
\text { single-user standalone installation with no } \\
\text { network connection. }\end{array}$ & 1.4 \\
\hline SC-003 & $\begin{array}{l}\text { The system shall not require user } \\
\text { authentication to operate. This } \\
\text { requirement does not apply to any } \\
\text { function that electronically transmits or } \\
\text { receives Submission data from Agency } \\
\text { systems. }\end{array}$ & 1.4 \\
\hline SC-004 & $\begin{array}{l}\text { Installation and use of the system shall } \\
\text { not require reconfiguration (i.e., changing } \\
\text { the default date format) of the target } \\
\text { computer. }\end{array}$ & 2.1.3.6 \\
\hline SC-005 & $\begin{array}{l}\text { The system shall be built such that it can } \\
\text { be translated into different languages. }\end{array}$ & 1.5 \\
\hline
\end{tabular}




\section{System Architecture (SA)}

\begin{tabular}{|c|c|c|}
\hline Reqmt-ID & Requirement & Design Elements \\
\hline SA-001 & $\begin{array}{l}\text { The system's web components (if any) } \\
\text { shall operate on computers running } \\
\text { appropriate popular browsers with a } \\
\text { secure transmission protocol. }\end{array}$ & N/A - No web components have been identified \\
\hline SA-002 & $\begin{array}{l}\text { The system shall support two user levels } \\
\text { of Entry and Submission development: }\end{array}$ & 2.2 \\
\hline SA-002.1 & $\begin{array}{l}\text { - Entry preparation by Contributors } \\
\text { (for example, at the operator or site } \\
\text { level). }\end{array}$ & 2.2 .2 \\
\hline SA-002.2 & $\begin{array}{l}\text { - Submission preparation at the SRA } \\
\text { level. }\end{array}$ & 2.2.1 \\
\hline SA-003 & $\begin{array}{l}\text { The system shall be able to accommodate } \\
\text { and apply different data structures for } \\
\text { different states in accordance with the } \\
\text { requirements of their specific AP } \\
\text { agreements. }\end{array}$ & 3.2 \\
\hline SA-003.1 & - $\quad$ Model Additional Protocol & 4.6.2 \\
\hline SA-003.2 & - $\quad$ United Kingdom & 4.6.7 \\
\hline SA-003.3 & - $\quad$ France & 4.6.4 \\
\hline SA-003.4 & - $\quad$ People’s Republic of China & 4.6.3 \\
\hline SA-003.5 & - $\quad$ Russia & 4.6.6 \\
\hline SA-003.6 & $\begin{array}{l}\text { - Other TBD (to be determined) } \\
\text { structures based upon future Agency } \\
\text { agreements }\end{array}$ & India: 4.6 .5 \\
\hline SA-004 & $\begin{array}{l}\text { The system shall allow the user to select } \\
\text { which data template the system will use } \\
\text { when creating Entries, Declarations, and } \\
\text { Submissions. }\end{array}$ & 3.2.2 \\
\hline SA-005 & $\begin{array}{l}\text { The system shall not require installation } \\
\text { of a separate database server. }\end{array}$ & APPENDIX B.4 \\
\hline
\end{tabular}




\section{Declarable Data (DD)}

\begin{tabular}{|c|c|c|}
\hline Reqmt-ID & Requirement & Design Elements \\
\hline DD-001 & $\begin{array}{l}\text { The system shall support the creation and } \\
\text { management of information that meets } \\
\text { the requirements set forth in Article } 2 \text { and } \\
\text { Article } 3 \text { of the AP with file attachments. }\end{array}$ & 3.3, 3.4 \\
\hline DD-002 & $\begin{array}{l}\text { A valid Entry is a collection of specific } \\
\text { information in a format that meets the } \\
\text { requirements of the AP article to which } \\
\text { the Entry applies. The required data } \\
\text { structure for each type of Entry is listed in } \\
\text { Appendix C of [the SRS]. }\end{array}$ & 4.6 \\
\hline DD-003 & $\begin{array}{l}\text { An Entry can exist independently without } \\
\text { a relationship or reference to any } \\
\text { Declaration or Submission. }\end{array}$ & $2.3 .2,2.4 .5,3.3$ \\
\hline DD-004 & $\begin{array}{l}\text { A Declaration consists of header } \\
\text { information and a collection of zero or } \\
\text { more entries that apply to a specific AP } \\
\text { article in a format that meets the } \\
\text { requirements of that article. The required } \\
\text { data structure for each type of Declaration } \\
\text { is listed in Appendix C [of the SRS]. }\end{array}$ & 4.6.2.2 \\
\hline DD-005 & $\begin{array}{l}\text { A valid Declaration is one that contains } \\
\text { all required header information (as } \\
\text { defined in Appendix C [of the SRS]) and } \\
\text { contains one and only one of the } \\
\text { following. } \\
\text { - An affirmative indication that it is a } \\
\text { "No Change" declaration, indicating } \\
\text { that the information provided in the } \\
\text { previously submitted active } \\
\text { declaration for the corresponding } \\
\text { article still stands, or } \\
\text { - An affirmative indication that there is } \\
\text { "Nothing to Declare" for the } \\
\text { associated article, or } \\
\text { A collection or list of one or more } \\
\text { valid Entries for the associated } \\
\text { article. }\end{array}$ & 4.6.2.2 \\
\hline DD-006 & $\begin{array}{l}\text { A Submission is a collection of one or } \\
\text { more Declarations that apply to a specific } \\
\text { Submission Type as defined in AP } \\
\text { Article 3, along with header information } \\
\text { in a format that meets the requirements of } \\
\text { that Submission Type. The required data } \\
\text { structure for each type of Submission is } \\
\text { listed in Appendix C of [the SRS]. }\end{array}$ & 4.6.2.2 \\
\hline DD-007 & $\begin{array}{l}\text { A Submission can exist independently, } \\
\text { without a relationship to or "knowledge" } \\
\text { of any Declaration or Entry. }\end{array}$ & 2.3.3, 2.4.15, 3.4 \\
\hline
\end{tabular}




\begin{tabular}{|c|c|c|}
\hline Reqmt-ID & Requirement & Design Elements \\
\hline DD-008 & $\begin{array}{l}\text { Submission content is identified in terms } \\
\text { of Article } 3 \text { of the Protocol Additional to } \\
\text { the Agreement(s) Between State(s) and } \\
\text { the International Atomic Energy Agency } \\
\text { for the Application of Safeguards. For } \\
\text { example, 3.a type Submission will expect } \\
\text { only Declarations identified under } \\
\text { Article 3.a and Notes. A detailed } \\
\text { description is provided in Appendix C of } \\
\text { the SRS]. }\end{array}$ & 4.6.2.2 \\
\hline DD-009 & $\begin{array}{l}\text { A valid Submission contains all required } \\
\text { header information (as defined in } \\
\text { Appendix C [of the SRS]) and contains a } \\
\text { collection of one or more Declarations } \\
\text { that meet the requirements of Article } 3 \text { for } \\
\text { that Submission Type. }\end{array}$ & 4.6.2.2 \\
\hline DD-010 & $\begin{array}{l}\text { Unless prescribed otherwise by a } \\
\text { Submission template, Declaration content } \\
\text { is identified in terms of Article } 2 \text { of the } \\
\text { Protocol Additional to the Agreement(s) } \\
\text { Between State(s) and the International } \\
\text { Atomic Energy Agency for the } \\
\text { Application of Safeguards. This includes } \\
\text { Article 2.c. A detailed description is } \\
\text { provided in Appendix C of [the SRS]. }\end{array}$ & 4.6.2.2 \\
\hline DD-011 & $\begin{array}{l}\text { The system shall be capable of tracking } \\
\text { Article 2.c support files, such as letters, } \\
\text { maps, and copies of correspondence (see } \\
\text { File Attachments for related } \\
\text { requirements). }\end{array}$ & 4.6.2.1, 4.6.2.2 \\
\hline DD-012 & $\begin{array}{l}\text { Data entry fields shall be displayed in } \\
\text { such a way as to easily distinguish } \\
\text { between declarable data fields that will be } \\
\text { submitted to the IAEA and internal data } \\
\text { fields that will not be submitted to the } \\
\text { IAEA, and the fields are reserved for } \\
\text { State use. }\end{array}$ & $2.4 .5,2.4 .15$ \\
\hline
\end{tabular}




\begin{tabular}{|c|c|c|}
\hline Reqmt-ID & Requirement & Design Elements \\
\hline ID-001 & $\begin{array}{l}\text { The system shall generate an internal } \\
\text { unique identifier for each Entry, } \\
\text { Declaration, and Submission. }\end{array}$ & 3.3.1, 3.4.1 \\
\hline ID-002 & $\begin{array}{l}\text { The system shall allow status information } \\
\text { to be tracked for Entry, Declaration, and } \\
\text { Submission (see Entry and Submission } \\
\text { Status section for related requirements). }\end{array}$ & $2.4 .5,2.4 .15,4.1$ \\
\hline ID-003 & $\begin{array}{l}\text { The system shall support the entry of } \\
\text { internal data at the Submission level. } \\
\text { These internal data are for use by State } \\
\text { authorities and are not included in the } \\
\text { submission to the IAEA. A detailed } \\
\text { description is provided in Appendix C of } \\
\text { [the SRS]. }\end{array}$ & 2.1.1, 2.4.15 \\
\hline ID-004 & $\begin{array}{l}\text { The system shall support the entry of } \\
\text { internal data at the Declaration level. } \\
\text { These internal data are for use by State } \\
\text { authorities and are not included in the } \\
\text { submission to the IAEA. A detailed } \\
\text { description is provided in Appendix C of } \\
\text { [the SRS]. }\end{array}$ & 2.1.1, 2.4.15 \\
\hline ID-005 & $\begin{array}{l}\text { The system shall support the entry of } \\
\text { internal data at the Entry level. These } \\
\text { internal data are for use by State } \\
\text { authorities and are not included in the } \\
\text { Submission to the IAEA. A detailed } \\
\text { description is provided in Appendix C of } \\
\text { [the SRS]. }\end{array}$ & $2.1 .1,2.4 .5$ \\
\hline
\end{tabular}

\section{Lookup Data (LD)}

\begin{tabular}{|l|l|c|}
\hline Reqme-ID & Requirement & Design Elements \\
\hline LD-001 & $\begin{array}{l}\text { The system shall load lookup files } \\
\text { containing choices for enumerated fields } \\
\text { provided by both the IAEA and the State. }\end{array}$ & 2.4.12, 3.7.2, 3.7.6 \\
\hline LD-002 & $\begin{array}{l}\text { The system shall allow the user to } \\
\text { manually update lookup tables not } \\
\text { provided by the IAEA. }\end{array}$ & 2.4.12, 2.4.6, 3.7.2, 3.7.4, 3.7.6 \\
\hline LD-003 & $\begin{array}{l}\text { The system shall allow the user to export } \\
\text { State-maintained lookup tables to a file } \\
\text { that can be loaded into another instance of } \\
\text { the system. }\end{array}$ & \\
\hline LD-004 & $\begin{array}{l}\text { A user shall be able to merge State- } \\
\text { maintained lookup data from a file with } \\
\text { existing local lookup data. }\end{array}$ & 3.7.3, 0 \\
\hline LD-005 & $\begin{array}{l}\text { The system shall allow users to view the } \\
\text { contents of lookup files and relevant } \\
\text { revision information. }\end{array}$ \\
\hline
\end{tabular}


Entry and Submission Statuses (ES)

\begin{tabular}{|c|c|c|}
\hline Reqmt-ID & Requirement & Design Elements \\
\hline ES-001 & $\begin{array}{l}\text { Entries shall have a Status field } \\
\text { representing their current state in the } \\
\text { declaration development process. }\end{array}$ & $2.4 .5,4.1 .3$ \\
\hline ES-002 & $\begin{array}{l}\text { Submissions shall have a Status field } \\
\text { representing their current state in the } \\
\text { declaration development process. }\end{array}$ & 2.4.15, 4.1.4 \\
\hline ES-003 & $\begin{array}{l}\text { There shall be at least two categories of } \\
\text { statuses: }\end{array}$ & 4.1.1, 4.1.3, 4.1.4 \\
\hline ES-003.1 & $\begin{array}{l}\text { Statuses representing in-development } \\
\text { declaration data which are fully } \\
\text { editable. }\end{array}$ & 4.1.1, 4.1.3, 4.1.4 \\
\hline ES-003.2 & $\begin{array}{l}\text { - } \\
\text { Statuses representing completed or } \\
\text { submitted declaration data which are } \\
\text { read only. }\end{array}$ & 4.1.1, 4.1.3, 4.1.4 \\
\hline ES-004 & $\begin{array}{l}\text { Users may change the status of an Entry or } \\
\text { Submission since the purpose of the status } \\
\text { field is for users to track their own } \\
\text { progress. }\end{array}$ & $2.4 .5,2.4 .15,4.1 .1$ \\
\hline ES-004.1 & $\begin{array}{l}\text { - Validation or other process } \\
\text { requirements shall not restrict status } \\
\text { changes. }\end{array}$ & 4.1.1, 4.6.1 \\
\hline ES-004.2 & $\begin{array}{l}\text { - Status may be changed automatically } \\
\text { by certain actions within the system. }\end{array}$ & 4.1.1 \\
\hline ES-004.3 & $\begin{array}{l}\text { - If a Submission has a completed or } \\
\text { submitted status, its Entries must also } \\
\text { be completed or submitted. }\end{array}$ & 4.1.1 \\
\hline
\end{tabular}

\section{Manage Submissions (MS)}

\begin{tabular}{|l|l|c|}
\hline Reqme-ID & Requirement & Design Elements \\
\hline MS-001 & $\begin{array}{l}\text { The system shall provide the capability to } \\
\text { create a new Submission. Submission } \\
\text { contents and properties are described in } \\
\text { Appendix C of [the SRS]. }\end{array}$ & 2.4.15, 3.4 \\
\hline MS-002 & $\begin{array}{l}\text { The system shall provide the capability to } \\
\text { create a new Submission by copying an } \\
\text { existing Submission. }\end{array}$ & 2.4.4, 3.4.1, 4.4.2 \\
\hline MS-003 & $\begin{array}{l}\text { The user shall be able to manage } \\
\text { Declarations as part of a Submission (see } \\
\text { Manage Declarations for related } \\
\text { requirements). }\end{array}$ & 2.4 .15 \\
\hline MS-004 & $\begin{array}{l}\text { The system shall provide the capability to } \\
\text { import a Submission (see Import/Export for } \\
\text { related requirements) from legacy formats. }\end{array}$ & 1.6, 2.4.16, 3.6 \\
\hline MS-005 & $\begin{array}{l}\text { The system shall provide the capability to } \\
\text { edit the properties of a Submission. }\end{array}$ & 2.4 .15 \\
\hline
\end{tabular}




\begin{tabular}{|l|l|l|}
\hline Reqme-ID & Requirement & Design Elements \\
\hline MS-006 & $\begin{array}{l}\text { The system shall provide a warning to the } \\
\text { user before deleting a Submission. }\end{array}$ & 2.4.4 \\
\hline MS-007 & $\begin{array}{l}\text { The system shall be capable of exporting a } \\
\text { Submission to a format that can be } \\
\text { submitted to the IAEA (see Import/Export } \\
\text { and Quality Control for related } \\
\text { requirements). }\end{array}$ & 1.6.2, 2.4.15 \\
\hline MS-008 & $\begin{array}{l}\text { The system shall allow a Submission to be } \\
\text { created based on the previous Submission, } \\
\text { with the possibility to denote each } \\
\text { declaration as “Update" or "No Change.” }\end{array}$ & 2.4.15, 3.4.1, 4.4.2 \\
\hline MS-009 & $\begin{array}{l}\text { When creating a new Submission to update } \\
\text { a previous Submission, the default reporting } \\
\text { period dates shall be generated } \\
\text { automatically. }\end{array}$ & 4.5.2, 4.5.3 \\
\hline
\end{tabular}

Manage Declarations (MD)

\begin{tabular}{|c|c|c|}
\hline Reqmt-ID & Requirement & Design Elements \\
\hline MD-001 & $\begin{array}{l}\text { The system shall provide the capability to } \\
\text { create a Declaration. Declaration contents } \\
\text { and properties are described in Appendix C } \\
\text { of [the SRS]. }\end{array}$ & 2.4.1, 2.4.15, 3.4 \\
\hline MD-002 & $\begin{array}{l}\text { The system shall allow a Declaration to be } \\
\text { created based on a previous Declaration, } \\
\text { with the possibility to denote it as "No } \\
\text { Change," or its Entries as "Update." }\end{array}$ & 2.4.15, 3.4.1, 4.4.2 \\
\hline MD-003 & $\begin{array}{l}\text { The user shall be able to manage Entries as } \\
\text { part of a Declaration (see Manage Entries } \\
\text { for related requirements). }\end{array}$ & 2.4.1, 2.4.15 \\
\hline MD-004 & $\begin{array}{l}\text { A user shall be able to add existing Entries } \\
\text { for a given Article to a Declaration for the } \\
\text { same Article. }\end{array}$ & 2.4.1, 2.4.15 \\
\hline MD-005 & $\begin{array}{l}\text { The user shall be able to extract Entries } \\
\text { from a Declaration, either to save } \\
\text { independently or insert into another } \\
\text { Declaration. }\end{array}$ & 2.4 .15 \\
\hline MD-006 & $\begin{array}{l}\text { A Declaration shall not be considered valid } \\
\text { unless it includes at least one Entry for the } \\
\text { associated Article or is marked as either } \\
\text { "No Change" or "Nothing to Declare." }\end{array}$ & 4.6.2.2 \\
\hline MD-007 & $\begin{array}{l}\text { A Declaration marked as "No Change" or } \\
\text { "Nothing to Declare" shall not contain } \\
\text { Entries. }\end{array}$ & 4.6.2.2 \\
\hline MD-008 & $\begin{array}{l}\text { The system shall provide the capability of } \\
\text { entering Declaration data as described in } \\
\text { Appendix C of [the SRS]. }\end{array}$ & 2.4.15, 3.4.2 \\
\hline
\end{tabular}




\begin{tabular}{|c|c|c|}
\hline Reqmt-ID & Requirement & Design Elements \\
\hline MD-009 & $\begin{array}{l}\text { The system shall provide the capability to } \\
\text { edit an existing Declaration. }\end{array}$ & 2.4.4, 2.4.15, 3.4.1 \\
\hline MD-010 & $\begin{array}{l}\text { The system shall provide the capability to } \\
\text { delete a Declaration. }\end{array}$ & 2.4 .15 \\
\hline MD-011 & $\begin{array}{l}\text { Where practical, the system shall use } \\
\text { structured fields in place of general text } \\
\text { fields that cannot be easily validated. }\end{array}$ & 4.6 \\
\hline MD-012 & $\begin{array}{l}\text { The system shall provide the capability of } \\
\text { attaching any number of files to a } \\
\text { Declaration (see File Attachments for } \\
\text { related requirements). }\end{array}$ & 2.4 .15 \\
\hline MD-013 & $\begin{array}{l}\text { Where practical, the layout of the primary } \\
\text { Declaration view shall resemble that } \\
\text { depicted in Guidelines and Format for } \\
\text { Preparation and Submission of } \\
\text { Declarations Pursuant to Articles } 2 \text { and } 3 \text { of } \\
\text { the Model Protocol Additional to } \\
\text { Safeguards Agreements (May 2004). }\end{array}$ & $2.1 .3,2.4 .15$ \\
\hline MD-013.1 & $\begin{array}{l}\text { The system shall provide the capability } \\
\text { of displaying sorted Entries within a } \\
\text { Declaration by Entry number or by } \\
\text { another column selected by the user. }\end{array}$ & 2.1.3, 2.4.15 \\
\hline MD-014 & $\begin{array}{l}\text { The system shall provide methods for } \\
\text { validating a Declaration for correctness and } \\
\text { completeness, and the user shall be able to } \\
\text { execute validation when editing or viewing } \\
\text { a Declaration. }\end{array}$ & $2.1 .5,2.4 .15,4.6$ \\
\hline MD-015 & $\begin{array}{l}\text { The process of validating a Declaration } \\
\text { shall also validate its Entries. }\end{array}$ & 4.6.1 \\
\hline MD-016 & $\begin{array}{l}\text { To avoid re-entry of the same data, the } \\
\text { system shall automatically generate default } \\
\text { reporting period and "as-of" dates where } \\
\text { practical. }\end{array}$ & 4.5 \\
\hline MD-017 & $\begin{array}{l}\text { Default reporting periods and "as-of" dates } \\
\text { should be easily changeable by the user. }\end{array}$ & 4.5 \\
\hline MD-018 & $\begin{array}{l}\text { If there are multiple Declarations in an } \\
\text { Initial Submission or the first update for a } \\
\text { given Article, then those Declarations may } \\
\text { have different "as-of" dates. }\end{array}$ & 4.6.2.2 \\
\hline
\end{tabular}


Manage Entries (ME)

\begin{tabular}{|c|c|c|}
\hline Reqmt-ID & Requirement & Design Elements \\
\hline ME-001 & $\begin{array}{l}\text { The system shall provide the capability to } \\
\text { create Entries. Entry data structure is } \\
\text { described in Appendix C of [the SRS]. }\end{array}$ & $2.4 .4,2.4 .5,3.3$ \\
\hline ME-002 & $\begin{array}{l}\text { The system shall provide the capability to } \\
\text { create a new Entry by copying an existing } \\
\text { Entry. }\end{array}$ & 2.4.4, 3.3.1, 4.4.2 \\
\hline ME-003 & $\begin{array}{l}\text { The system shall allow the user to include } \\
\text { any number of Entries in a Declaration. }\end{array}$ & 2.4 .15 \\
\hline ME-004 & $\begin{array}{l}\text { The system shall provide the capability to } \\
\text { edit an existing Entry. }\end{array}$ & 2.4.4, 2.4.5, 3.3.1 \\
\hline ME-005 & $\begin{array}{l}\text { The system shall provide the capability to } \\
\text { edit a previously submitted Entry by } \\
\text { changing the status of the Entry (or the } \\
\text { Submission containing it) to one that allows } \\
\text { editing. }\end{array}$ & 2.4.5, 4.1.3 \\
\hline ME-006 & $\begin{array}{l}\text { The system shall allow an Entry to be } \\
\text { created based on a previous Entry, with the } \\
\text { possibility to denote it as "Update” or "No } \\
\text { Change.” }\end{array}$ & 2.4.4, 3.4.1, 4.4.2 \\
\hline ME-006.1 & $\begin{array}{l}\text { The system shall display a warning } \\
\text { before allowing the user to create an } \\
\text { Update Entry for a previously } \\
\text { submitted Final Entry. }\end{array}$ & 4.4 .2 \\
\hline ME-007 & $\begin{array}{l}\text { The system shall provide the capability to } \\
\text { delete an Entry. }\end{array}$ & 2.4 .4 \\
\hline ME-008 & $\begin{array}{l}\text { The system shall provide the capability of } \\
\text { displaying Entries sorted by a column } \\
\text { selected by the user. }\end{array}$ & 2.1.3.2 \\
\hline ME-009 & $\begin{array}{l}\text { Where practical, the system shall use } \\
\text { structured fields in place of general text } \\
\text { fields that cannot be easily validated. }\end{array}$ & 4.6 \\
\hline ME-010 & $\begin{array}{l}\text { The system shall provide the capability of } \\
\text { attaching any number of files to an Entry } \\
\text { (see File Attachments for related } \\
\text { requirements). }\end{array}$ & 2.4 .5 \\
\hline ME-011 & $\begin{array}{l}\text { The system shall provide the same functions } \\
\text { for managing an Entry whether or not it is } \\
\text { part of a Declaration. }\end{array}$ & 2.4.5, 2.4.15, 3.3.1 \\
\hline ME-012 & $\begin{array}{l}\text { The system shall provide methods for } \\
\text { validating an Entry for correctness and } \\
\text { completeness, and the user shall be able to } \\
\text { execute validation when editing or viewing } \\
\text { an Entry. }\end{array}$ & $2.1 .5,2.4 .5,4.6$ \\
\hline ME-013 & $\begin{array}{l}\text { It shall be possible to transfer Entries to } \\
\text { other users. }\end{array}$ & 3.13 .2 \\
\hline
\end{tabular}




\begin{tabular}{|l|l|l|}
\hline Reqmt-ID & Requirement & Design Elements \\
\hline ME-014 & $\begin{array}{l}\text { If Entries are not stored in individual files } \\
\text { by default, it shall be possible to export and } \\
\text { import them to and from the file system. }\end{array}$ & \multirow{2}{*}{ 4.13.2 } \\
\hline ME-015 & $\begin{array}{l}\text { The system shall allow input of multiple } \\
\text { fuel cycle stages for Article 2.a.(i), 2.a.(x), } \\
\text { and 2.b.(i) Entries. }\end{array}$ & \\
\hline ME-016 & $\begin{array}{l}\text { For Article 2.a.(ix) Entries, the system shall } \\
\text { allow only the year or only the year and } \\
\text { month to be entered in case the exact export } \\
\text { date is not available. }\end{array}$ & \\
\hline
\end{tabular}

\section{Declaration and Entry Numbering (DN)}

\begin{tabular}{|l|l|l|}
\hline Reqme-ID & Requirement & Design Elements \\
\hline DN-001 & $\begin{array}{l}\text { By default, the first declaration created in } \\
\text { the system shall be numbered “1.” } \\
\text { Subsequent declarations shall be numbered } \\
\text { sequentially within each submission and } \\
\text { shall continue sequentially from one } \\
\text { submission to the next. }\end{array}$ & \\
\hline DN-002 & $\begin{array}{l}\text { By default, the system shall automatically } \\
\text { renumber Declarations as they are added to, } \\
\text { removed from, or re-ordered within a } \\
\text { Submission to achieve sequential } \\
\text { numbering with no gaps. }\end{array}$ & \\
\hline DN-003 & $\begin{array}{l}\text { It shall be possible to specify the starting } \\
\text { number for automatically numbering } \\
\text { declarations within a submission. This may } \\
\text { be used to specify a number other than “1” } \\
\text { for the first Declaration in the first } \\
\text { Submission. }\end{array}$ & \\
\hline DN-004 & $\begin{array}{l}\text { By default, the system shall automatically } \\
\text { renumber Entries as they are added to, } \\
\text { removed from, or re-ordered within a } \\
\text { Declaration to achieve sequential } \\
\text { numbering with no gaps. }\end{array}$ & \\
\hline
\end{tabular}

\section{Entry Cross-referencing (EC)}

\begin{tabular}{|l|l|l|}
\hline Reqmt-ID & Requirement & Design Elements \\
\hline EC-001 & $\begin{array}{l}\text { The system shall provide the capability for } \\
\text { an Entry to cross-reference any number of } \\
\text { other Entries, either within the same and/or } \\
\text { another Submission. }\end{array}$ & \\
\hline EC-002 & $\begin{array}{l}\text { The system shall provide the capability for } \\
\text { an Entry to cross-reference any number of } \\
\text { Declarations within the same and/or another } \\
\text { Submission. }\end{array}$ & \\
\hline EC-003 & $\begin{array}{l}\text { A cross-reference shall consist of the } \\
\text { Declaration and Entry Number of the } \\
\text { related entry. }\end{array}$ & 2.4 .5 \\
\hline
\end{tabular}




\begin{tabular}{|c|c|c|}
\hline Reqmt-ID & Requirement & Design Elements \\
\hline EC-004 & $\begin{array}{l}\text { Within the same Submission, if Entry "A" } \\
\text { refers to Entry "B,” then Entry "B” shall } \\
\text { automatically refer to Entry “A." }\end{array}$ & 2.1 .8 \\
\hline EC-005 & $\begin{array}{l}\text { Where applicable, the system shall perform } \\
\text { quality check to ensure that if Entry “A” } \\
\text { references Entry "B,” Entry "B” references } \\
\text { Entry “A” as well. }\end{array}$ & 4.6.2.3 \\
\hline EC-006 & $\begin{array}{l}\text { The system shall support viewing the } \\
\text { referenced Entry from the referencing Entry } \\
\text { if the referenced Entry is available. }\end{array}$ & 2.1 .8 \\
\hline EC-007 & It shall be possible to delete a Reference. & 2.4 .5 \\
\hline EC-008 & $\begin{array}{l}\text { If Declarations or Entries within a } \\
\text { Submission are renumbered, all References } \\
\text { within that Submission shall be updated to } \\
\text { reflect the new numbers. }\end{array}$ & 4.3 \\
\hline
\end{tabular}

Data Entry (DE)

\begin{tabular}{|c|c|c|}
\hline Reqmt-ID & Requirement & Design Elements \\
\hline DE-001 & $\begin{array}{l}\text { Data validation shall be available where } \\
\text { practical on all entry screens [see } \\
\text { Quality Control (QC) for related } \\
\text { requirements]. }\end{array}$ & 2.1.5, 2.4.5, 2.4.15, 4.6 \\
\hline DE-002 & $\begin{array}{l}\text { Data entry fields shall be displayed in such } \\
\text { a way as to easily distinguish between } \\
\text { which elements the IAEA considers } \\
\text { mandatory and those considered optional. }\end{array}$ & 2.1 .1 \\
\hline DE-003 & $\begin{array}{l}\text { Printable output of Declarations or } \\
\text { Submissions not intended for paper transfer } \\
\text { to the IAEA shall include the designation, } \\
\text { "Not valid for submission to the IAEA until } \\
\text { certified by the State." }\end{array}$ & 2.1 .7 \\
\hline DE-004 & $\begin{array}{l}\text { Where appropriate (such as for authorities } \\
\text { and addresses), the system shall provide a } \\
\text { means for States to manage their own } \\
\text { validation lists. }\end{array}$ & 2.4.12, 3.7.2, 0, 3.7.6 \\
\hline DE-005 & $\begin{array}{l}\text { Where data are displayed in a grid, the } \\
\text { system shall allow the user to change the } \\
\text { column order and width. These changes } \\
\text { shall persist between user sessions. }\end{array}$ & 2.1.3.2 \\
\hline
\end{tabular}

Search, Filter, and Navigate (SFN)

\begin{tabular}{|l|l|l|}
\hline Reqmt-ID & Requirement & Design Elements \\
\hline SFN-001 & $\begin{array}{l}\text { At a minimum, the system shall provide the } \\
\text { capability of searching Declaration data } \\
\text { using the following criteria: }\end{array}$ & 3.5 \\
\hline
\end{tabular}




\begin{tabular}{|l|l|c|}
\hline Reqmi-ID & Requirement & Design Elements \\
\hline SFN-001.1 & $-\quad$ Declaration number & 3.5 \\
\hline SFN-001.2 & $-\quad$ AP Article & 3.5 \\
\hline SFN-001.3 & $-\quad$ Declaration date(s) & 3.5 \\
\hline SFN-001.4 & $-\quad$ Cross-reference & 3.5 \\
\hline SFN-002 & $\begin{array}{l}\text { The system shall include the capability of } \\
\text { performing a full-text search (including } \\
\text { Boolean operators and wildcards) across all } \\
\text { data that is accessible by the system. }\end{array}$ & 3.5 \\
\hline SFN-003 & $\begin{array}{l}\text { It shall be possible to easily filter, navigate, } \\
\text { and browse Declaration and Entry data } \\
\text { throughout a Submission. }\end{array}$ & 2.4 .15 \\
\hline
\end{tabular}

\section{Import/Export (IE)}

\begin{tabular}{|l|l|c|}
\hline Reqmi-ID & Requirement & Design Elements \\
\hline IE-001 & $\begin{array}{l}\text { The system shall be capable of importing } \\
\text { files of text-delimited Submissions as } \\
\text { defined in Protocol Reporter 1.0 export } \\
\text { format even if the file to be imported } \\
\text { includes validation errors. }\end{array}$ & 3.6.1 \\
\hline IE-002 & $\begin{array}{l}\text { The system shall be capable of importing } \\
\text { files of text-delimited Submissions as } \\
\text { defined in Protocol Reporter 2.0 export } \\
\text { format even if the file to be imported } \\
\text { includes validation errors. }\end{array}$ & \\
\hline IE-003 & $\begin{array}{l}\text { The system shall be capable of importing } \\
\text { files of .xml Submissions as defined in } \\
\text { Protocol Reporter 2.0 export format even if } \\
\text { the file to be imported includes validation } \\
\text { errors. }\end{array}$ & \\
\hline IE-004 & $\begin{array}{l}\text { The system must be capable of importing } \\
\text { previously submitted Declaration data } \\
\text { provided by the Agency in PR1, PR2, or } \\
\text { PR3 format. }\end{array}$ & 3.6 .1 \\
\hline IE-005 & $\begin{array}{l}\text { By default, legacy data shall be imported in } \\
\text { read-only format. }\end{array}$ & \multirow{2}{*}{3.6 .1} \\
\hline IE-006 & $\begin{array}{l}\text { A user may opt to convert legacy data into } \\
\text { an editable format. }\end{array}$ & 2.6 .1 \\
\hline
\end{tabular}




\begin{tabular}{|c|c|c|}
\hline Reqmt-ID & Requirement & Design Elements \\
\hline IE-007 & $\begin{array}{l}\text { The system shall notify the user and provide } \\
\text { details if an import or export fails. }\end{array}$ & 3.6 .2 \\
\hline IE-008 & $\begin{array}{l}\text { The system must be capable of deleting any } \\
\text { imported data. }\end{array}$ & 3.6 .2 \\
\hline IE-009 & $\begin{array}{l}\text { The ability to import and export files shall } \\
\text { be a standard feature of the system and not } \\
\text { require special activation to enable. }\end{array}$ & 3.13 \\
\hline IE-010 & $\begin{array}{l}\text { The system shall be capable of importing } \\
\text { any output exported by the system. }\end{array}$ & 3.13 \\
\hline IE-011 & $\begin{array}{l}\text { All data exported by the system shall be in } \\
\text { human-readable format. }\end{array}$ & 3.13 .1 \\
\hline IE-012 & $\begin{array}{l}\text { The system shall support export in UTF-8 } \\
\text { text format. }\end{array}$ & 3.13 .1 \\
\hline IE-013 & $\begin{array}{l}\text { Data shall be exported without adding to or } \\
\text { changing its content. }\end{array}$ & 3.13 .2 \\
\hline IE-014 & $\begin{array}{l}\text { Where applicable, exported data shall } \\
\text { include all attached files (see File } \\
\text { Attachments (FA) for related } \\
\text { requirements). }\end{array}$ & 3.13.2, 3.13.3 \\
\hline IE-015 & $\begin{array}{l}\text { Where applicable, exported Submissions } \\
\text { shall provide dates formatted as } \\
\text { YYYY-MM-DD. }\end{array}$ & 2.1.3.6 \\
\hline IE-016 & $\begin{array}{l}\text { All data shall be validated prior to export. } \\
\text { The user shall be notified of any errors and } \\
\text { warnings but may choose to continue the } \\
\text { export. }\end{array}$ & 2.4 .6 \\
\hline
\end{tabular}

\section{File Attachments (FA)}

\begin{tabular}{|l|l|c|}
\hline Reqmt-ID & Requirement & Design Elements \\
\hline FA-001 & $\begin{array}{l}\text { The system shall include the capability to } \\
\text { attach any number of files at the Submission } \\
\text { level. }\end{array}$ & 2.4 .15 \\
\hline FA-002 & $\begin{array}{l}\text { The system shall include the capability to } \\
\text { attach any number of files at the Declaration } \\
\text { level. }\end{array}$ & 2.4 .15 \\
\hline FA-003 & $\begin{array}{l}\text { The system shall include the capability to } \\
\text { attach any number of files at the Entry level. }\end{array}$ & 2.4 .5 \\
\hline FA-004 & $\begin{array}{l}\text { The system shall include the capability of } \\
\text { removing attached files. }\end{array}$ & $2.4 .5,2.4 .15$ \\
\hline
\end{tabular}




\begin{tabular}{|l|l|l|}
\hline Reqmt-ID & Requirement & Design Elements \\
\hline FA-005 & $\begin{array}{l}\text { The system shall enable the user to open } \\
\text { attachments with associated applications } \\
\text { defined in the operating system. }\end{array}$ & 2.1 .9 \\
\hline
\end{tabular}

\section{Quality Control (QC)}

\begin{tabular}{|l|l|l|}
\hline Reqmt-ID & Requirement & Design Elements \\
\hline QC-001 & $\begin{array}{l}\text { The system shall employ validation checks } \\
\text { and user feedback mechanisms wherever } \\
\text { necessary to help ensure all Entry, } \\
\text { Declaration, and Submission data meet the } \\
\text { format and content requirements specified } \\
\text { in the Detail Requirements and Appendix C } \\
\text { [of the SRS]. }\end{array}$ & \\
\hline QC-002 & $\begin{array}{l}\text { Each validation feedback message shall be } \\
\text { identified as an error, warning, or } \\
\text { informational message to indicate the } \\
\text { severity of the problem. }\end{array}$ & 2.1.5, 2.4.5, 2.4.15, 4.6 \\
\hline QC-003 & $\begin{array}{l}\text { Validation feedback shall contain sufficient } \\
\text { detail to help the user correct the problem. }\end{array}$ & \\
\hline QC-004 & $\begin{array}{l}\text { Failed validation shall not prevent the use of } \\
\text { any functionality other than the final export } \\
\text { to the Agency. }\end{array}$ & \\
\hline QC-005 & $\begin{array}{l}\text { Where applicable, the system shall perform } \\
\text { quality checks on dates to ensure } \\
\text { consistency and correct chronology. }\end{array}$ & \\
\hline QC-006 & $\begin{array}{l}\text { Where applicable, the system shall perform } \\
\text { quality checks on Entry cross-references } \\
\text { and other data to ensure consistency and } \\
\text { accuracy. }\end{array}$ & \\
\hline QC-007 & $\begin{array}{l}\text { If applicable, the system shall provide the } \\
\text { user with details of any errors or messages } \\
\text { returned from IAEA following electronic } \\
\text { transmission of a Submission. }\end{array}$ & \\
\hline
\end{tabular}

\section{Reports (RP)}

\begin{tabular}{|l|l|l|}
\hline Reqmi-ID & Requirement & Design Elements \\
\hline RP-001 & $\begin{array}{l}\text { The system shall provide the capability to } \\
\text { generate a viewable and printable report of } \\
\text { any Submission, including its Declarations } \\
\text { and Entries (or any user-selected subset). } \\
\text { Detailed descriptions of such reports are } \\
\text { provided in Appendix D of [the SRS]. }\end{array}$ & \\
\hline RP-002 & $\begin{array}{l}\text { The system shall provide the capability to } \\
\text { generate a viewable and printable report of } \\
\text { Submission due dates. A detailed } \\
\text { description of the report is provided in } \\
\text { Appendix D of [the SRS]. }\end{array}$ & 2.5.2, 2.5.4 \\
\hline
\end{tabular}




\begin{tabular}{|l|l|c|}
\hline Reqme-ID & Requirement & Design Elements \\
\hline RP-003 & $\begin{array}{l}\text { The system shall support multiple print } \\
\text { layouts. }\end{array}$ & 2.1 .7 \\
\hline RP-004 & $\begin{array}{l}\text { Where applicable, the system shall clearly } \\
\text { differentiate Declarable Data from Internal } \\
\text { Data in reports. }\end{array}$ & $2.5 .2,2.5 .4$ \\
\hline RP-005 & $\begin{array}{l}\text { The system shall provide the capability to } \\
\text { generate a viewable and printable report of } \\
\text { a user-selected collection of one or more } \\
\text { Entries. A detailed description of the report } \\
\text { is provided in Appendix D of [the SRS]. }\end{array}$ & 2.5 .2 \\
\hline
\end{tabular}

\section{User Documentation (UD)}

\begin{tabular}{|l|l|l|}
\hline Reqmt-ID & Requirement & Design Elements \\
\hline UD-001 & $\begin{array}{l}\text { System help shall include thorough } \\
\text { documentation, explanation of major system } \\
\text { functions and reference documents. }\end{array}$ & TBD \\
\hline UD-001.1 & $\begin{array}{l}\text { System help shall include a copy of the } \\
\text { IAEA document Model Protocol } \\
\text { Additional to the Agreement(s) Between } \\
\text { State(s) and the International Atomic } \\
\text { Energy Agency for the Application of } \\
\text { Safeguards (INFCIRC/540, corrected). }\end{array}$ & TBD \\
\hline UD-001.2 & $\begin{array}{l}\text { System help shall include a copy of the } \\
\text { IAEA document Guidelines and } \\
\text { Format for Preparation and } \\
\text { Submission of Declarations Pursuant to } \\
\text { Articles 2 and 3 of the Model Protocol } \\
\text { Additional to Safeguards Agreements } \\
\text { (or equivalent document) }\end{array}$ & TBD \\
\hline UD-001.3 & $\begin{array}{l}\text { System help shall include an } \\
\text { explanation of the determination of due } \\
\text { dates, including the significance of the } \\
\text { Entry Into Force and As-Of dates. }\end{array}$ & \\
\hline UD-002 & $\begin{array}{l}\text { Where applicable, access to system help } \\
\text { topics shall be context-sensitive. }\end{array}$ & TBD \\
\hline UD-003 & $\begin{array}{l}\text { Where necessary, help topics shall include } \\
\text { examples of Entry, Declaration, and } \\
\text { Submission data. }\end{array}$ & TBD \\
\hline
\end{tabular}



APPENDIX D. SRS ADDENDUM 



\section{APPENDIX D. SRS ADDENDUM}

In the process of developing the SDS and the visual prototype, several changes to information in the SRS became necessary.

\section{D.1 Glossary and Acronyms}

The term "SRA" now refers to "State or Regional Authority" rather than "Safeguards Regulatory Authority.”

\section{D.2 Requirements}

The highest-level validation errors should now prevent transmission of Submissions to the Agency. This changes the following sections of the SRS that state validation errors should not prevent export or transmission:

- $\quad$ Use Cases 3.7.1 and 3.7.2

- Requirements IE-016, and QC-004

The requirement to explicitly reserve numbers for particular Declarations and Entries was removed as the Entry and Declaration Numbering rules were developed for the SDS (Sect. 4.3). This removes requirement DN-005.

\section{D.3 Data Structures}

All State lookup fields should be replaced with String fields. When entering a value in these fields, a user may select either a value form the State lookup list or type in a new value. (The exact wording of the State name may change between Submissions.)

The Location and Sublocation data types should be combined. (Multiple Locations may be covered by the same Entry, leading to confusion as to which Sublocations relate to which Locations.) This involves the following changes:

- $\quad$ Add the Building, Room, Subarea, Facility/LOF, and Managed Access fields to the Location data type.

- $\quad$ Remove Sublocation fields wherever they appear in Entries.

In the following Entries, the Location fields should be changed to accept multiple Location values:

- Model AP 2.a.(i), 2.a.(iv), 2.a.(v) Mine/Plan, 2.a.(vi)(a), 2.a.(vi)(c), 2.a.(viii) Processing, 2.a.(ix)(a), 2.b.(i), and 2.b.(ii)

- $\quad$ AP for France 2.a.(i) and 2.a.(iii)

- $\quad$ AP for Russia 2.a.(i) and 2.a.(iii)

All other Location fields should still accept only one Location value.

A "Facility/LOF Identifier" composite data type should be defined to be used in Model AP 2.a.(iii) Entries as follows: 


\section{Facility/LOF Identifier}

Used to identify either a Facility or LOF by a combination of codes.

\begin{tabular}{|l|l|l|}
\hline Name & Type & Notes \\
\hline Facility Code & String & Must not be filled in if LOF Code is filled in. \\
\hline LOF Code & String & Must not be filled in if Facility Code is filled in. \\
\hline MBA Code & String & Should only be filled in if LOF Code is filled in. \\
\hline KMP Code & String & Must be filled in if MBA Code is filled in. \\
\hline LOC ID & String & Should only be filled in if KMP Code is filled in. \\
\hline
\end{tabular}

The Facility, LOF, MBA, and KMP fields in Model AP 2.a.(iii) Entry should be replaced with a set of Facility/LOF Identifier objects.

The "Site ID" field in Model AP 2.a.(iii) Entry should be replaced with the "Site Name" field. This field should be of type String, but it can have options provided from the Sites lookup list.

The "Estimated Annual Capacity" field should be removed from Model AP 2.a.(v) State Total Entry.

The "Element” field in Model AP 2.a.(vii) Entry should be renamed to "Material.”

The "Declaration Date” field in Submission should be renamed to "Submission Date."

The "Site Code" field in Model AP 2.a.(iii) Declaration Details should be replaced with "Site Code" and "Site Name" fields. Both fields should be of type String, but they can have options provided from the Site Code lookup list.

Remove the Annex I Voluntary Expansion and Annex II Voluntary Expansion lookup lists. These lists should be replaced with an Agency-provided Nuclear Suppliers Group Guidelines lookup list.

The "Correction” value should be added to the Reference Type lookup table.

Model AP 3.c, 3.d., and 3.e Submissions should not be referred to as "Updates." For now, we will use the term "Declarations" instead (e.g., "Annual declaration" or "Quarterly declaration" rather than "Annual update” or "Quarterly update”). This should also affect the description for equivalent Submission Types in State-specific data templates.

The AP for India only has one Submission Type: General. 
APPENDIX E. PR3 VISUAL PROTOTYPE OVERVIEW 



\section{APPENDIX E. PR3 VISUAL PROTOTYPE OVERVIEW}

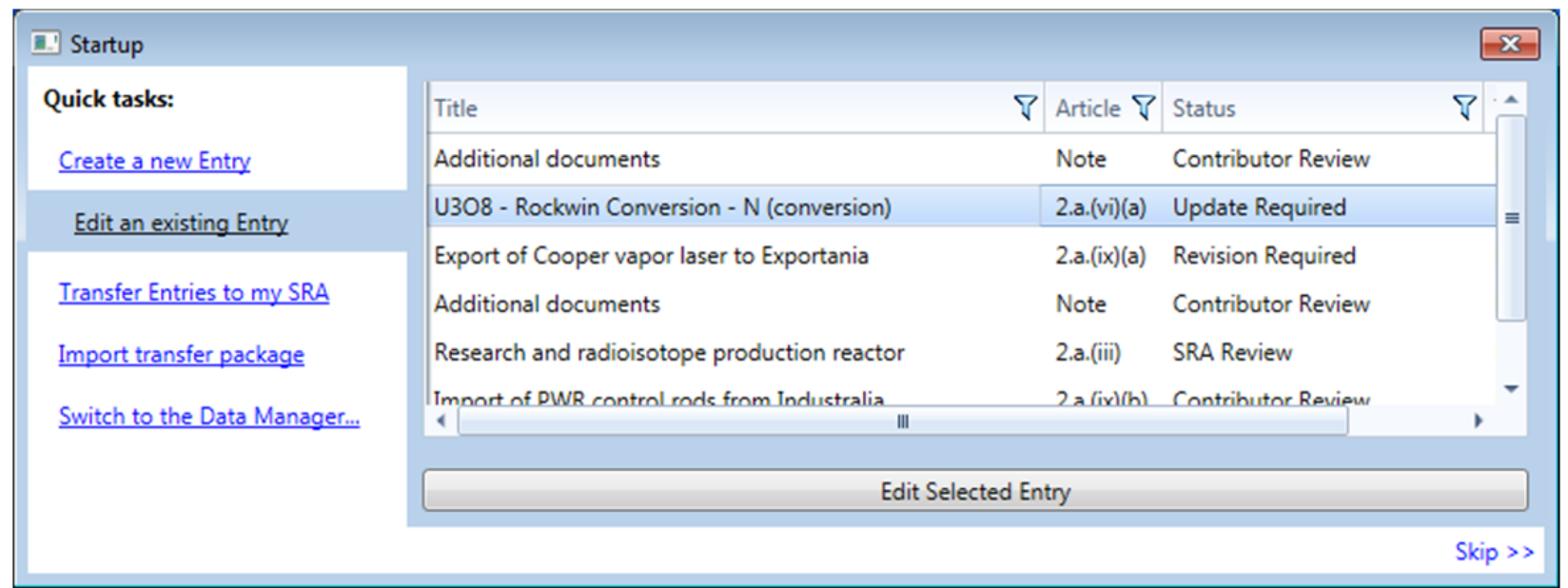

Fig. E.1. A mockup of a Startup window. In addition to Contributor and SRA Modes, a simplified menu of the most basic Contributor tasks could be presented on first launch. Advanced users could skip to the Data Manager.

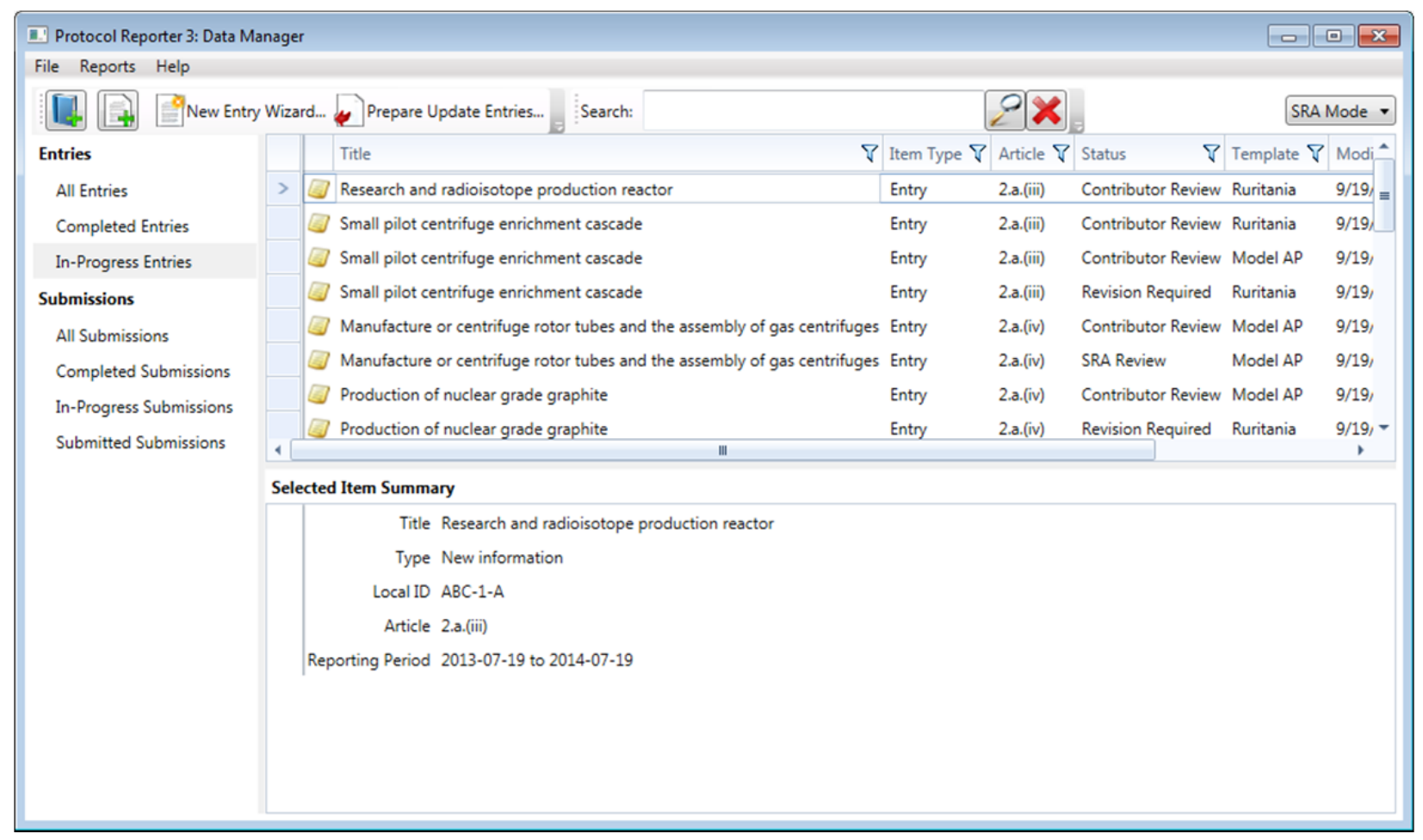

Fig. E.2. A mockup of the Data Manager window. Users could manage their database of Entries and/or Submissions, as well as access all other PR3 functions. (Sect. 2.4.4) 


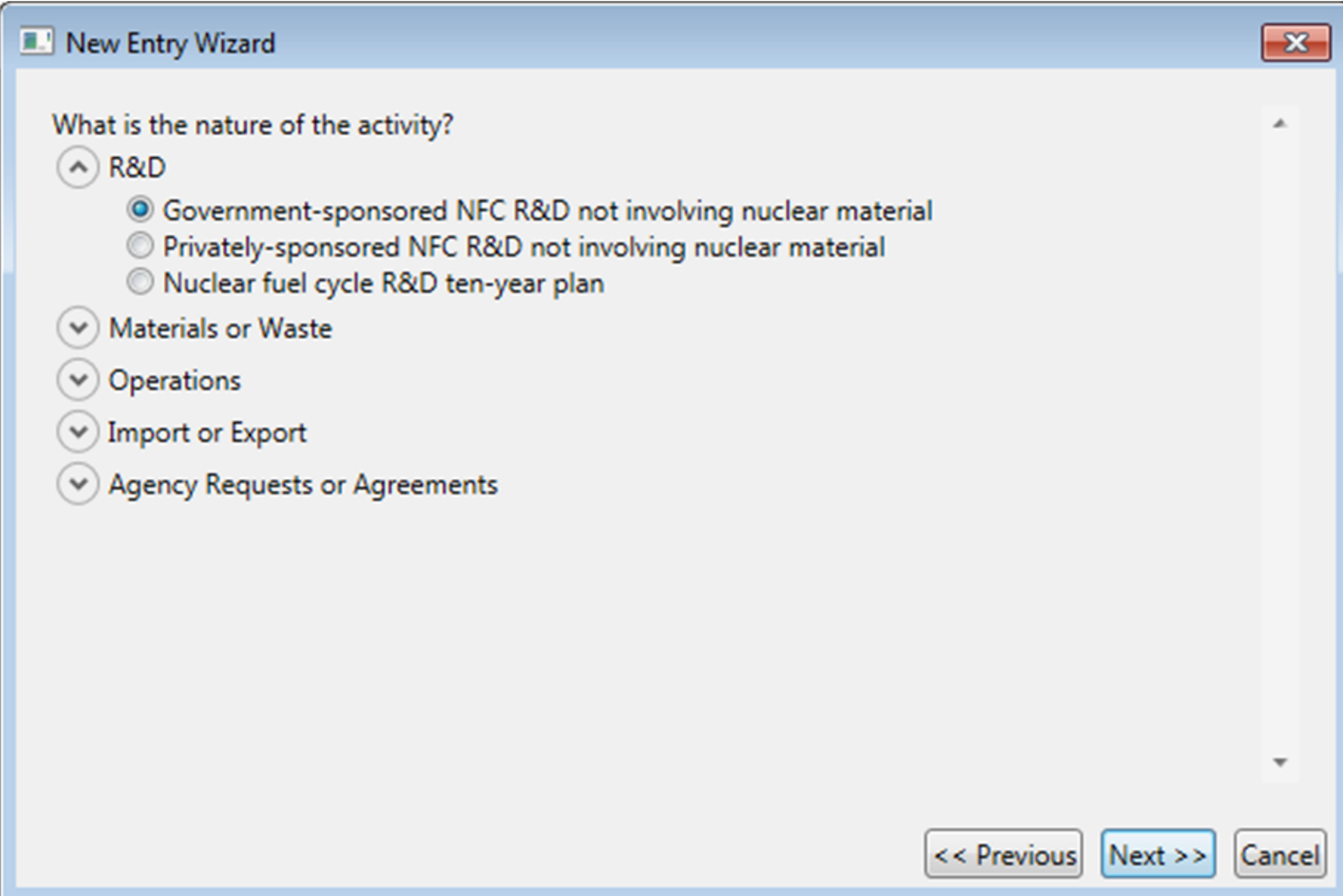

Fig. E.3. A mockup of a New Entry Wizard. This tool would guide users with limited AP-specific knowledge though the process of creating new Entries. (Sect. 4.4.1) 


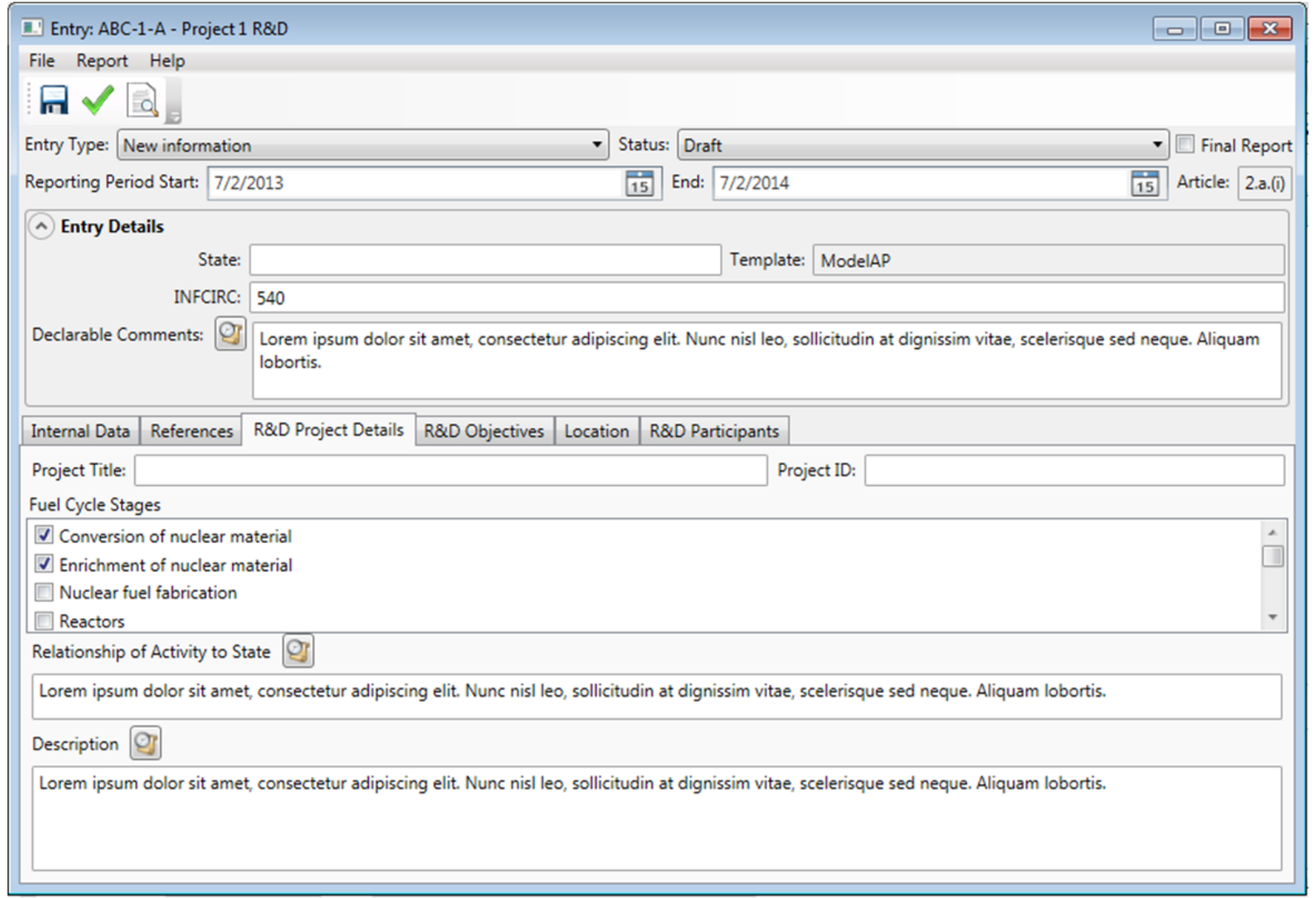

Fig. E.4. A mockup of the Entry Editor. This should combine common fields (such as type, status, internal comments, and references) with article-specific fields provided by the data template. (Sect. 2.4.5)

\begin{tabular}{|c|c|c|c|c|c|c|}
\hline \multicolumn{6}{|c|}{ ㅍ. Messages } & 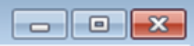 \\
\hline \multicolumn{7}{|c|}{\begin{tabular}{|l|l|} 
E Errors (2) I Warnings (1) I Info (1) \\
\end{tabular}} \\
\hline & & Category $\nabla$ & Message & Type $\nabla$ & Location & \\
\hline \multirow[t]{4}{*}{$>$} & i & ModelAP 2.a.(i) & No Project Title was entered & Entry & RAPA Isotope Separation - Phase II & \\
\hline & 1 & Entry & No Local ID was entered. & Entry & $\underline{\text { Rurifert Ltd., U plant, operating }}$ & \\
\hline & 3 & ModelAP 2.a.(i) & No Fuel Cycle Stages were selected. & Entry & RAPA Isotope Separation - Phase II & \\
\hline & 3 & ModelAP 3.a & Submissions should contain at least one Declaration. & Submission & $\underline{\text { Initial Submission }}$ & \\
\hline
\end{tabular}

Fig. E.5. A mockup of Validation Messages. Functions that provided validation such as the Entry Editor, Submission Editor, Agency Export, and Declaration Data Manager would use a common interface for displaying results. 


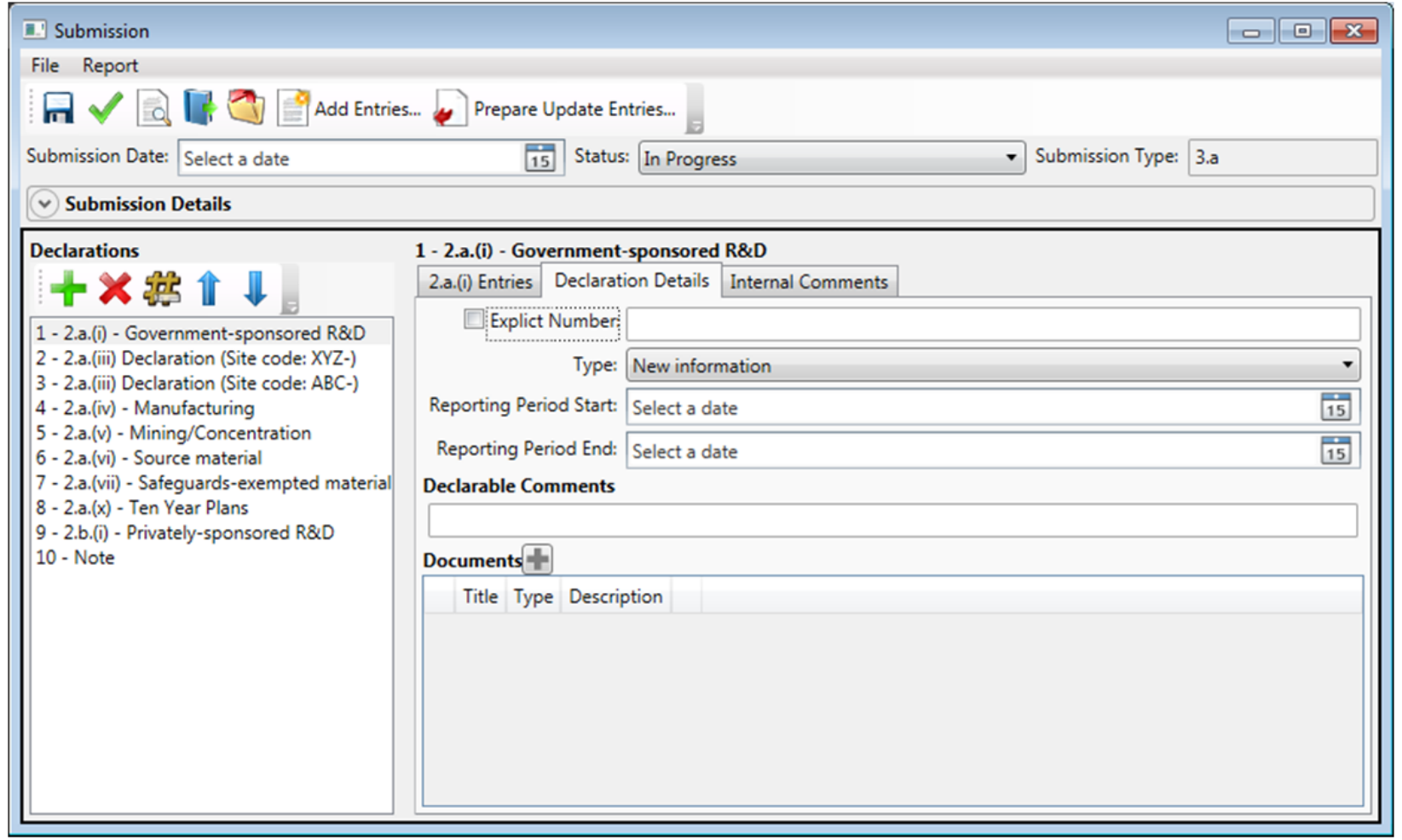

Fig. E.6. A mockup of the Submission Editor. This should allow to edit Submission fields as well as Declaration and Entries contained by the Submission. (Sect. 2.4.15)

ㄹ. Add Entries to Submission

Eligible Entries (25) Ineligible Entries (10)

Selected Entries: 3

Title

$\checkmark$ Additional documents

$\checkmark$ Ten year plan: Multi-unit nuclear power station

$\checkmark$ Ten year plan: Multi-unit nuclear power station

$\square$ Fuel fabrication and testing facility

U3O8 - Rockwin Conversion - N (conversion)

CSR Laser development

Various (U) - Ruritania aggregates (ceramics)

Design study on advanced dry or wet reprocessing system

Ten year plan: Uranium exploration in South Kings Province

Design study on advanced dry or wet reprocessing system

Small pilot centrifuge enrichment cascade

Manufacture or centrifuge rotor tubes and the assembly of gas centrifuges

Ten year plan: Uranium exploration in South Kings Province

Heavy water production

Research and radioisotope production reactor

Ten year plan: Uranium exploration in South Kings Province
Local ID Article Contributor

T-QC-858 Note ABC Corporation

H-NX-631 2.a. $(x) \quad A B C$ Corporation

S-FZ-242 2.a.(x) ABC Corporation

A-MA-820 2.a.(iii) ABC Corporation

B-AE-636 2.a.(vi)(a) ABC Corporation

Q-E8-630 2.b.(i) ABC Corporation

Z-DU-770 2.a.(vi)(a) ABC Corporation

E-P6-234 2.b.(i) ABC Corporation

H-A7-389 2.a.(x) ABC Corporation

J-R0-127 2.b.(i) ABC Corporation

K-DQ-991 2.a.(iii) ABC Corporation

$A B C$ Corporation

$A B C$ Corporation

$A B C$ Corporation

$A B C$ Corporation

$A B C$ Corporation

Fig. E.7. A mockup of the Add Entries to Submission window. This is used to select specific individual Entries from the user's database to a Submission. (Sect. 2.4.1) 


\begin{tabular}{|c|c|c|c|c|c|c|}
\hline \multicolumn{4}{|c|}{ I. Report Viewer: Due Dates Report } & \multirow{2}{*}{\multicolumn{2}{|c|}{ 口回 }} & \multirow[t]{2}{*}{28} \\
\hline $14 \quad 4 \quad 1$ & 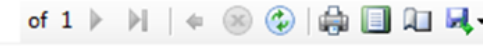 & $100 \%$ & Find | Next & & & \\
\hline$\Gamma$ & & \multirow{2}{*}{$\begin{array}{l}\text { Entry Into Force } \\
\text { Report Dates: }\end{array}$} & $7 / 29 / 2012$ & & & $\hat{=}$ \\
\hline & & & $7 / 29 / 2014-7 / 29 / 2016$ & & & \\
\hline Submission & Description & Due Date & Due Date Rule & & & \\
\hline 3.d & Quarterly update (Annex II exports) & $8 / 29 / 2014$ & $\begin{array}{l}\text { Within } 60 \text { days after the end of } \\
\text { each quarter }\end{array}$ & & & $\equiv$ \\
\hline 3.d & Quarterly update (Annex II exports) & $8 / 29 / 2014$ & $\begin{array}{l}\text { Within } 60 \text { days after the end of } \\
\text { each quarter }\end{array}$ & & & \\
\hline 3.d & Quarterly update (Annex II exports) & $11 / 29 / 2014$ & $\begin{array}{l}\text { Within } 60 \text { days after the end of } \\
\text { each quarter }\end{array}$ & & & \\
\hline 3.d & Quarterly update (Annex II exports) & $11 / 29 / 2014$ & $\begin{array}{l}\text { Within } 60 \text { days after the end of } \\
\text { each quarter }\end{array}$ & & & \\
\hline 3.d & Quarterly update (Annex II exports) & $3 / 1 / 2015$ & $\begin{array}{l}\text { Within } 60 \text { days after the end of } \\
\text { each quarter }\end{array}$ & & & \\
\hline 3.d & Quarterly update (Annex II exports) & $3 / 1 / 2015$ & $\begin{array}{l}\text { Within } 60 \text { days after the end of } \\
\text { each quarter }\end{array}$ & & & \\
\hline 3.b & Annual update for initial & $5 / 15 / 2015$ & by May 15 covering previous year & & & \\
\hline 3.c & $\begin{array}{l}\text { Annual update (source material imports \& } \\
\text { exports) }\end{array}$ & $5 / 15 / 2015$ & by May 15 covering previous year & & & \\
\hline 3.e & $\begin{array}{l}\text { Annual update (intermediate and high-level } \\
\text { waste) }\end{array}$ & $5 / 15 / 2015$ & $\begin{array}{l}180 \text { days before further } \\
\text { processing is carried out, and by } \\
\text { May } 15 \text { covering previous year }\end{array}$ & & & \\
\hline 3.d & Quarterly update (Annex II exports) & $5 / 30 / 2015$ & $\begin{array}{l}\text { Within } 60 \text { days after the end of } \\
\text { each quarter }\end{array}$ & & & \\
\hline $3 d$ & Quartorly undato (Annov II ovnarte) & $5 / 30 / 2015$ & Wlithin for dave aftor the and of & & & 5 \\
\hline
\end{tabular}

Fig. E.8. A mockup of the Report Viewer window displaying a Due Dates report. The Report Viewer should allow a user to export or print a report. (Sect. 2.5)

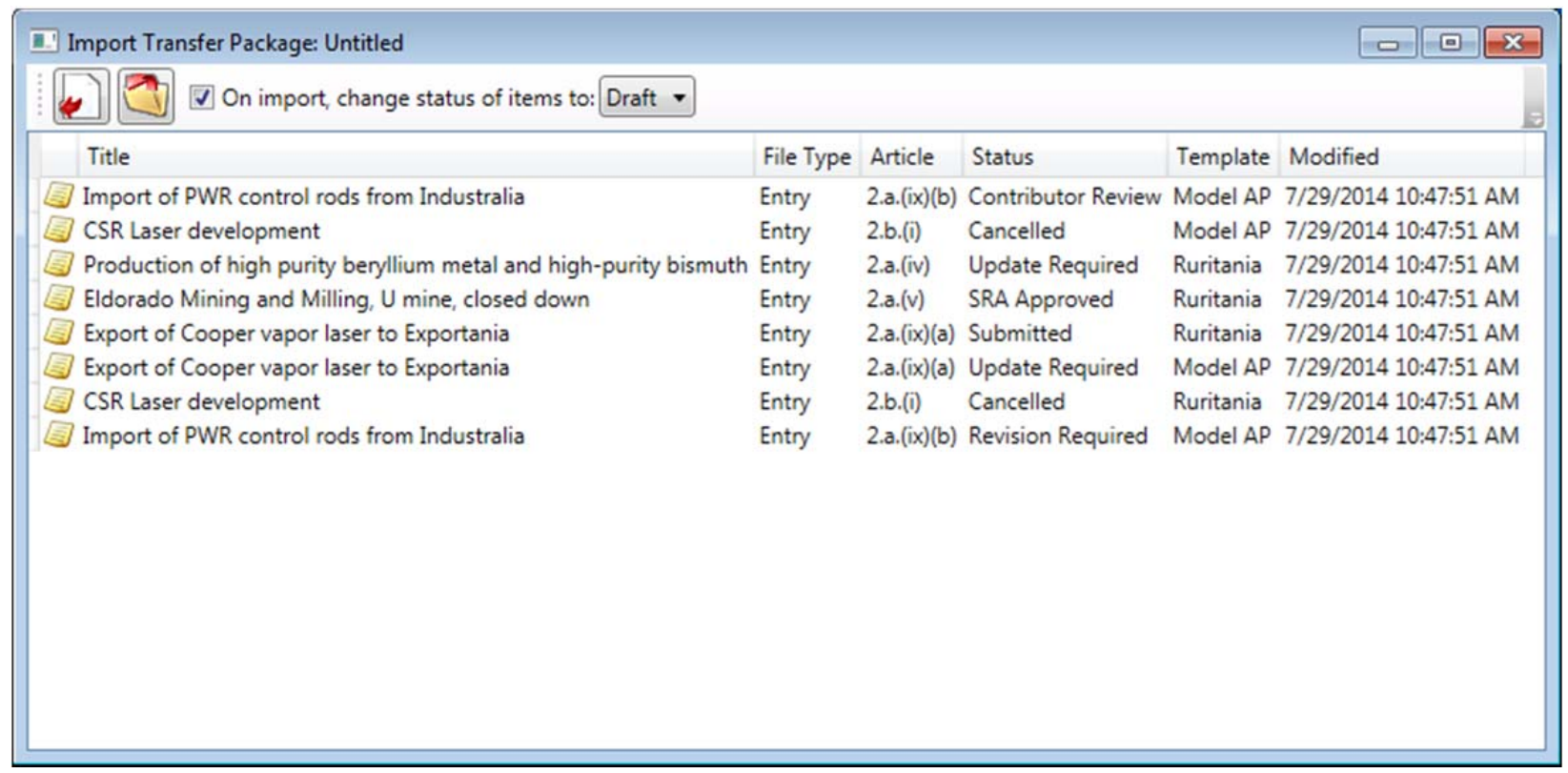

Fig. E.9. A mockup of the Transfer Package manager. A user may export selected Entries or Submissions from his or her database as a Transfer Package file or import selected Entries or Submission from a Transfer Package into his or her database. (Sects. 2.4.6, 2.4.7) 


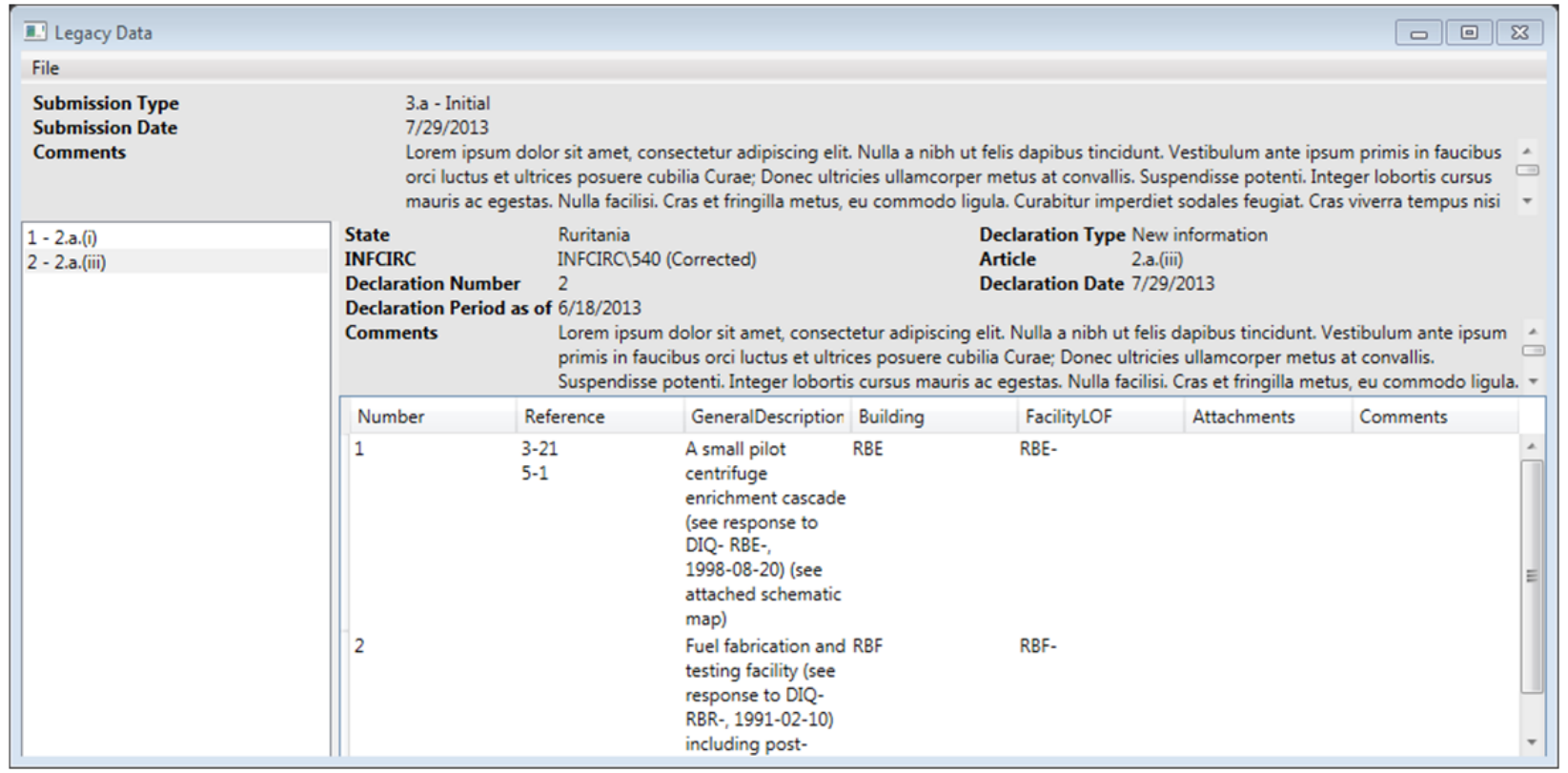

Fig. E.10. A mockup of the Legacy Data view window. A user may view the contents of existing PR1/PR2 exports and convert them to PR3 format. (Sect. 2.4.16) 